\title{
The Use Of Myeloid Suppressor Cells To Inhibit Experimental Autoimmune Encephalomyelitis: A Potential Immunotherapy For Multiple Sclerosis
}

\author{
BY \\ Clare Yan Slaney
}

\begin{abstract}
A thesis
submitted to the Victoria University of Wellington in fulfilment of the requirements for the degree of Doctor of Philosophy in Biomedical Science
\end{abstract}

Victoria University of Wellington 2009 


\begin{abstract}
The ideal treatments for multiple sclerosis (MS) are ones that specifically target the disease causing autoreactive T cells without compromising the immune system's ability to respond to pathogens and infections. However, the current treatments for MS are antigen non-specific and there is a need for the development of antigen-specific therapies that do not induce global immunosuppression. Thus, this thesis aims to investigate the potential of using the body's own suppressor cells to develop an antigen-specific immunotherapy to inhibit experimental autoimmune encephalomyelitis (EAE), the murine model for MS.
\end{abstract}

In our laboratory, there are two versions of mutated superantigens, SMEZ-2-M1 (SM) and double mutant SMEZ-2 (DM). SM is defective at its TCR binding site, but retains its ability to bind to MHCII molecules. Based on previous findings from our laboratory that administration of a SM conjugate with myelin oligodendrocyte glycoprotein $\left(\mathrm{MOG}_{35-55}\right)$ peptide in incomplete Freund's adjuvant (IFA) suppressed EAE in a CD25 $5^{+}$ regulatory $\mathrm{T}$ cell (Treg)-dependent manner, it was hypothesised that the administration

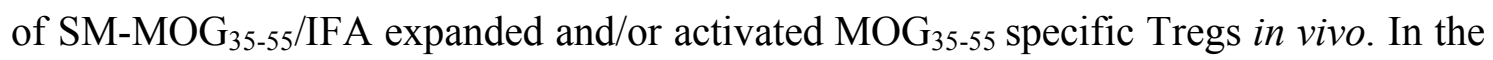
first part of this thesis, I tested this hypothesis. The experimental results showed that

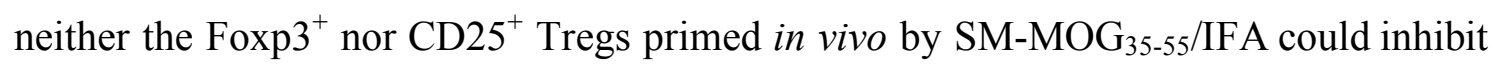
EAE and surprisingly, treating mice with $\mathrm{SM}-\mathrm{MOG}_{35-55} / \mathrm{IFA}$ did not significantly suppress EAE as previously described.

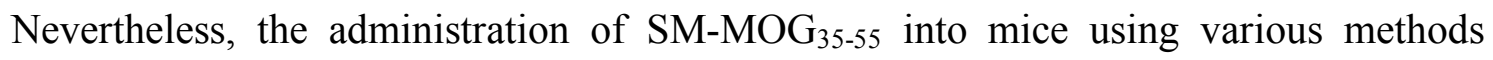
repeatedly showed minor suppression of EAE, suggesting an in vivo suppressive capability of $\mathrm{SM}_{-} \mathrm{MOG}_{35-55}$. Interestingly, after being injected into mice intravenously, $\mathrm{SM}$ was captured by a blood $\mathrm{MHCII}^{-} \mathrm{CD} 11 \mathrm{~b}^{+} \mathrm{F} 4 / 80^{+} \mathrm{Gr}-1^{+}$cell population in an MHCII-independent manner. Cells expressing the same surface markers have been reported in the literature to be myeloid derived suppressor cells (MDSCs), suggesting that the $\mathrm{SM}^{+} \mathrm{MHCII} C \mathrm{CD} 11 \mathrm{~b}^{+} \mathrm{F} 4 / 80^{+} \mathrm{Gr}-1^{+}$cells may be suppressor cells, i.e. a

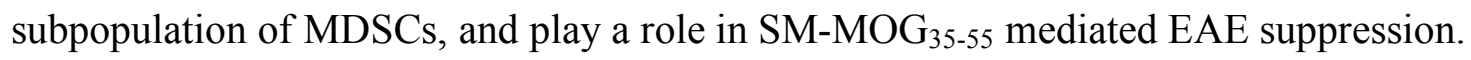

In the second part of this thesis, I went on to test the blood $\mathrm{MHCII}^{-} \mathrm{CD} 11 \mathrm{~b}^{+} \mathrm{F} 4 / 80^{+} \mathrm{Gr}-1^{+}$ cells' suppressive potential using DM. Unlike SM, DM is defective at both MHCII and 
TCR binding sites, and possessed an enhanced binding capability to the blood $\mathrm{MHCII}^{-}$ $\mathrm{CD} 11 \mathrm{~b}^{+} \mathrm{F} 4 / 80^{+} \mathrm{Gr}-1^{+}$cells. The experimental results demonstrated that the blood $\mathrm{MHCII}^{-} \mathrm{CD} 11 \mathrm{~b}^{+} \mathrm{F} 4 / 80^{+} \mathrm{Gr}-1^{+}$cells are potent suppressors of $\mathrm{T}$ cell responses, and were subsequently named as blood MDSCs (bMDSCs). bMDSCs suppressed $\mathrm{T}$ cell proliferation in vitro in a cell contact-dependant manner, and nitric oxide played an important role in this suppression.

In the third part of this thesis, I investigated the potential of using DM for EAE suppression via bMDSCs. When DM was conjugated to $\mathrm{MOG}_{35-55}$ and administered subcutaneously into mice, EAE was suppressed in a $\mathrm{MOG}_{35-55}$-specific manner. Moreover, the adoptive transfer of bMDSCs from the DM-MOG $35-55$ treated mice transferred EAE suppression, confirming that bMDSCs play an important role in this suppression.

Taken together, these results reveal a previously unknown role of bMDSCs in limiting immune responses. Moreover, the use of DM to direct the activity of bMDSC may prove to be a unique antigen-specific immunotherapy for EAE, which has great potential to be developed into a treatment of MS and other autoimmune diseases. 


\section{Acknowledgements}

I wish to express my sincere gratitude to my supervisor Dr. Thomas Bäckström, for his guidance, encouragement and support during my $\mathrm{PhD}$. His positive outlook and scientific insight inspired me and gave me confidence. I benefited greatly from his advice, constructive criticism and comments, particularly when exploring new ideas.

This work would not have been possible without the support and encouragement from my co-supervisor, Dr. Jacquie Harper, under whose supervision I finished the experimental work and the thesis. I would like to specially thank her for giving me great advice in how to structure this thesis. She ploughed through the text of this thesis and provided very helpful and encouraging feedback.

Dr. Anne La Flamme, my co-supervisor, has also been abundantly helpful through the duration of my $\mathrm{PhD}$ and has assisted me in numerous ways, including giving me guidance on a number of critical experiments, providing scientific directions and constructive feedback on my thesis.

I am very grateful to everyone at the Malaghan Institute of Medical Research, for providing a supportive environment and help on many occasions. I would like to specially thank Aras Toker and Evelyn Spittle for their invaluable technical support, helpful discussions, encouragement and good company. Completing this work would have been more difficult were it not for the support and friendship provided by all at Malaghan Institute.

Thanks to Professor John Fraser and his team for providing me with the modified superantigens that were critical for this project.

I have greatly appreciated the financial support provided by the Lottery Health Research Committee, Health Research Council of New Zealand and the Wellington Medical Research Foundation.

I am also thankful to my husband Dr. David Slaney, my parents, Dusty and friends for their limitless support and encouragement. It is to them that I dedicate this work. 


\section{Table of contents}

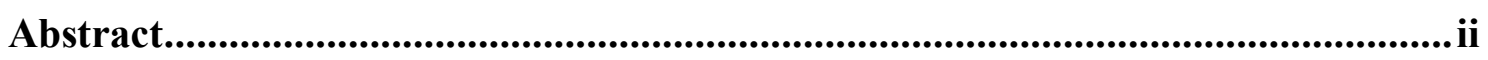

Acknowledgements .................................................................................................... iv

Table of contents...................................................................................................

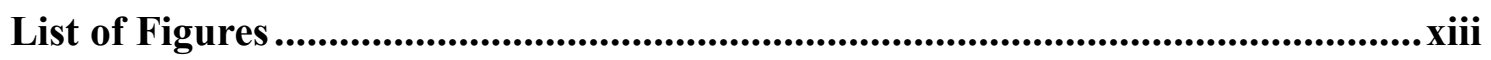

List of Tables .....................................................................................................

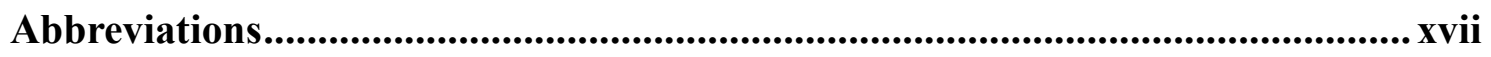

Chapter 1: General Introduction ........................................................................1

1.1 The mammalian immune system .....................................................................2

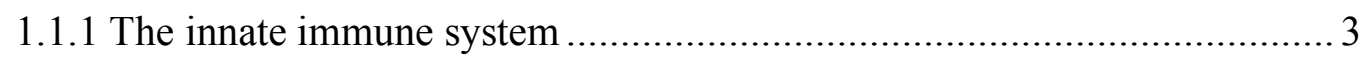

1.1.1.1 Toll like receptors ...................................................... 3

1.1.1.2 Cells of the innate immune system ....................................... 4

1.1.2 The adaptive immune system..................................................... 4

1.1.2.1 Antigen presenting cells ................................................... 5

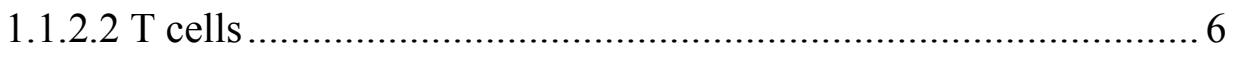

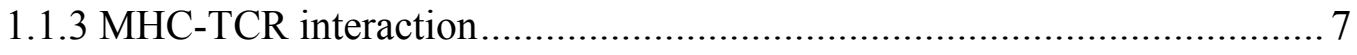

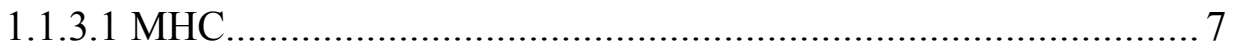

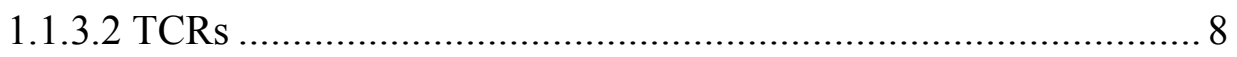

1.1.3.3 MHC-TCR interaction ..................................................... 8

1.1.3.4 Co-stimulation ............................................................ 9

1.2 Immunological tolerance............................................................................. 10

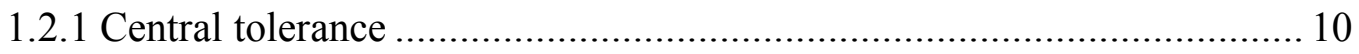

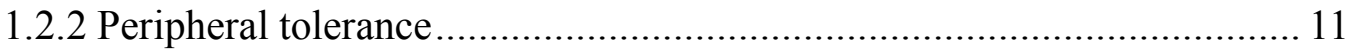

1.3 Regulatory $T$ cells.............................................................................................. 12

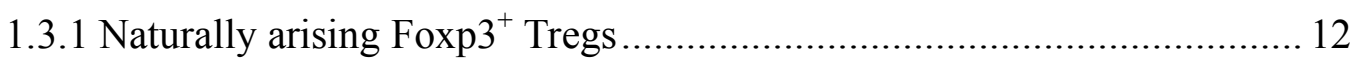

1.3.2 Mechanisms of Foxp $3^{+}$nTreg mediated suppression............................ 13

1.3.3 Interaction between Tregs and APC................................................. 13

1.4 Myeloid derived suppressor cells ............................................................ 14

1.5 Autoimmune disease.............................................................................................. 15

1.6 Multiple sclerosis ................................................................................................. 16

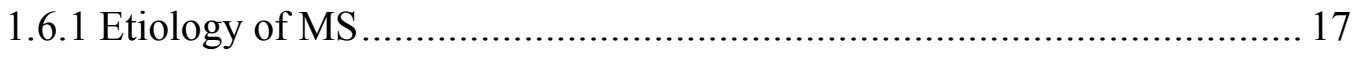

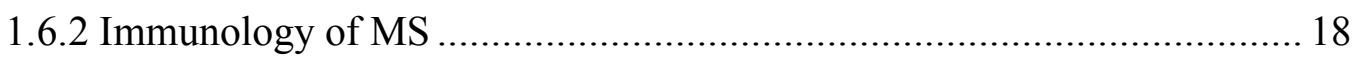




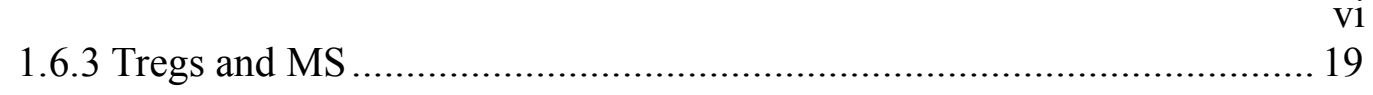

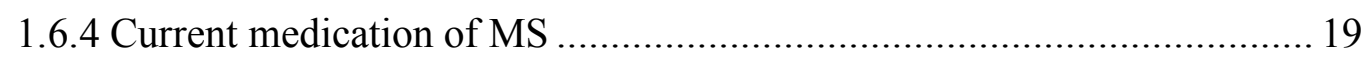

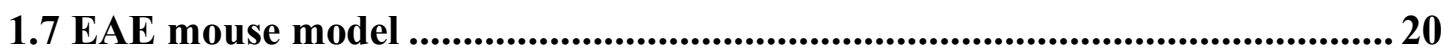

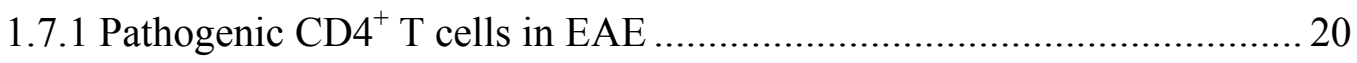

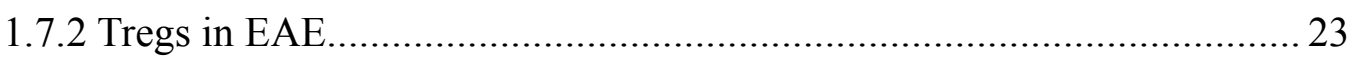

1.7.3 Suppressive myeloid cells in EAE .................................................. 23

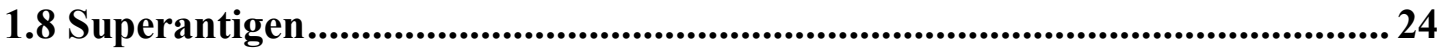

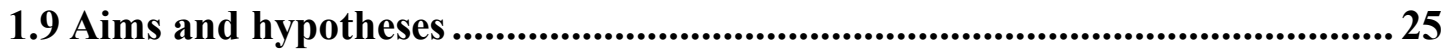

Chapter 2: Materials and Methods................................................................................. 27

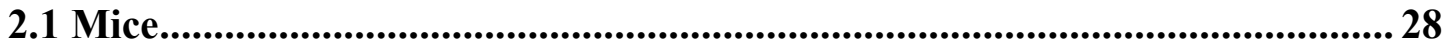

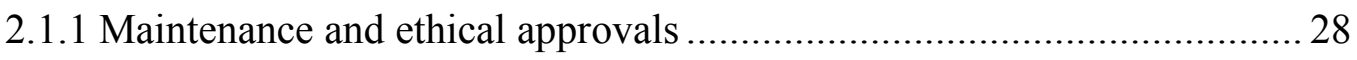

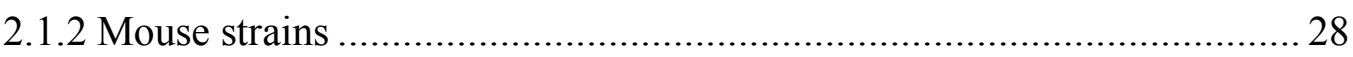

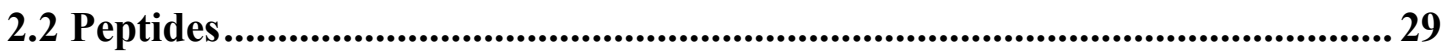

2.3 Conjugation of peptides to mSAg …....................................................................... 30

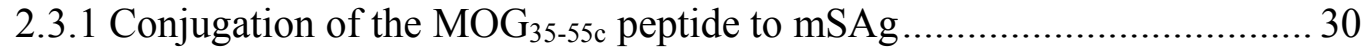

2.3.2 Conjugation of the $\mathrm{OVA}_{323-339 \mathrm{c}}$ peptide to $\mathrm{mSAg}$............................... 30

2.4 Immunisations for EAE suppression ........................................................... 31

2.4.1 EAE induction and clinical evaluation............................................... 31

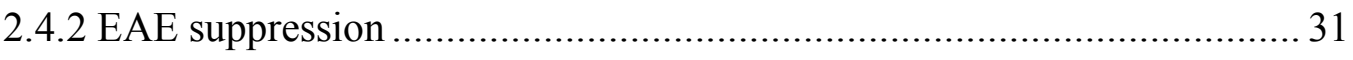

2.4.2.1 Subcutaneous treatment ...................................................... 32

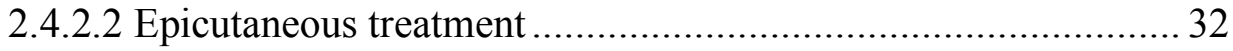

2.4.2.3 Adoptive transfer of cells ................................................... 32

2.4.3 Statistical analysis for EAE suppression ........................................... 32

2.5 Histology

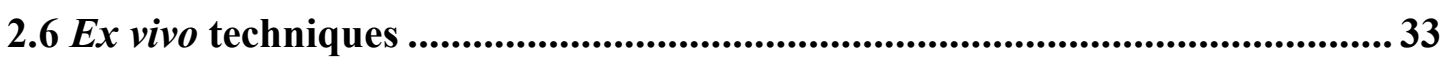

2.6.1 Preparation of single cell suspension from lymphoid tissue .................. 33

2.6.2 Preparation of single cell suspension from the blood ............................ 33

2.6.3 Preparation of single cell suspension from the CNS ............................ 34

2.6.4 Preparation of single cell suspension from the peritoneal cavity............. 34

2.6.5 Preparation of single cell suspension from the liver ............................ 34

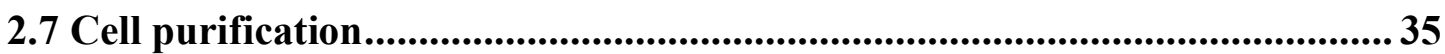

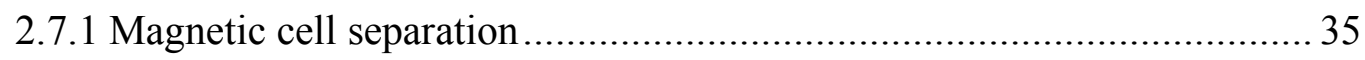

2.7.1.1 MACS purification of $\mathrm{CD}^{+}$and $\mathrm{CD} 8^{+} \mathrm{T}$ cells ....................... 35

2.7.1.2 MACS purification of $\mathrm{CD} 4^{+} \mathrm{CD} 25^{+} \mathrm{T}$ cells and $\mathrm{CD} 4^{+} \mathrm{CD} 25^{-}$ 
vii

2.7.1.3 Two step purification of the blood CD11 ${ }^{+}{ }^{+} \mathrm{Ly} 6 \mathrm{G}^{-}$cells .............. 36

2.7.1.4 Depletion of $\mathrm{MHCII}^{+}$cells using BioMag beads........................ 37

2.7.1.5 Purification of $\mathrm{CD}^{+}$cells using Dynabeads .............................. 37

2.7.1.6 Purification of $\mathrm{CD} 11 \mathrm{c}^{+}$cells from the spleen.................................. 38

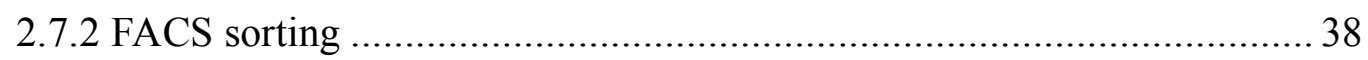

2.8 Bone marrow dendritic cell culture .....................................................................38

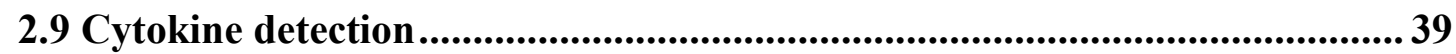

2.10 Fluorescent labelling of cells and analysis by flow cytometry.......................39

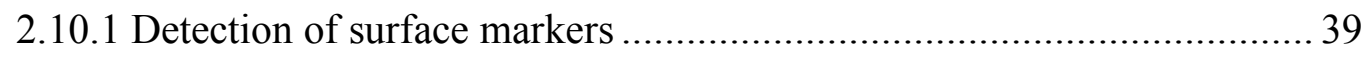

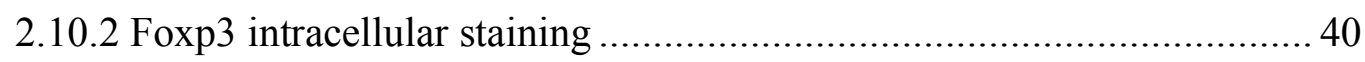

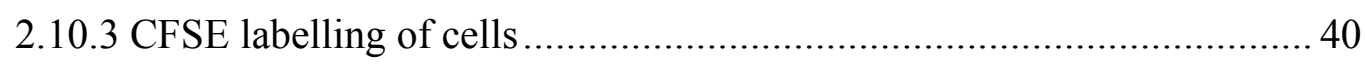

2.10.4 Detecting apoptotic and necrotic cells.................................................... 41

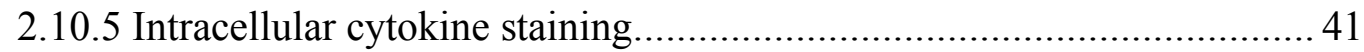

2.11 In vitro cell functional assays........................................................................... 42

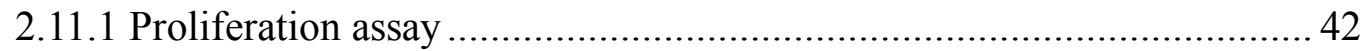

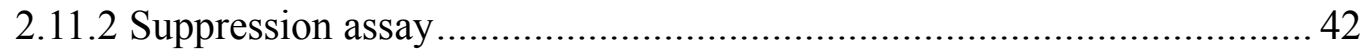

2.11.2.1 $\left[{ }^{3} \mathrm{H}\right]$-thymidine uptake based suppression assay....................... 42

2.11.2.2 CFSE dilution based suppression assay .................................. 43

2.11.3 Statistical analysis for cell proliferation and suppression....................... 43

2.12 Morphological stain for cells ........................................................................... 43

2.13 Confocal microscopy …........................................................................................ 44

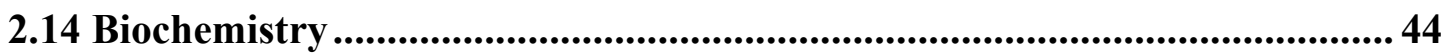

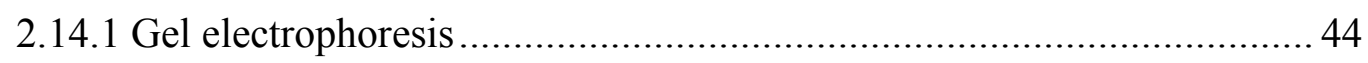

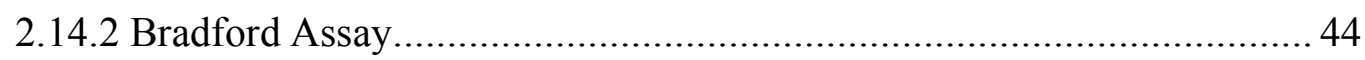

2.14.3 Labelling proteins by using fluorophore or biotin ................................. 45

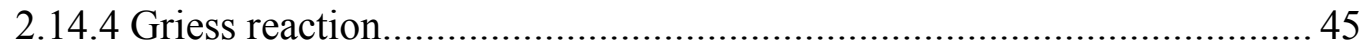

Chapter 3: SM-MOG ${ }_{35-55}$ Mediated EAE Suppression ............................................... 46

3.1 Introduction ............................................................................................................ 47

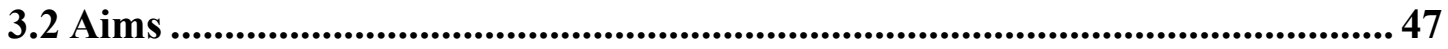

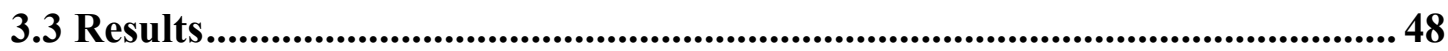

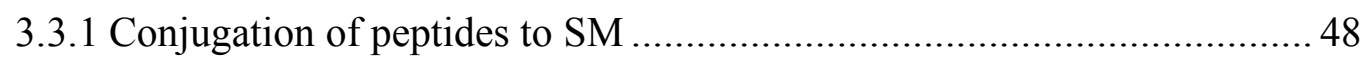

3.3.1.1 Peptides could be efficiently conjugated to SM ......................... 48

3.3.1.2 Conjugation of peptide to SM improved efficiency of the 
peptide presentation to $\mathrm{CD} 4^{+} \mathrm{T}$ cells in vitro.

3.3.1.3 Conjugation of peptide to SM improved efficiency of the peptide presentation to $\mathrm{CD} 4^{+} \mathrm{T}$ cells in vivo.......................... 50

3.3.2 SM-MOG $\mathrm{MO}_{35-55} / \mathrm{IFA}$ treatment did not suppress EAE ............................ 51

3.3.2.1 SM-MOG ${ }_{35-55} /$ IFA treatment did not suppress EAE via

Foxp3 $3^{+}$Tregs

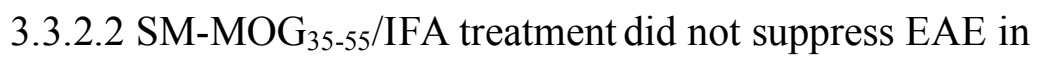

Foxp3-GFP KI mice 53

3.3.2.3 SM-MOG ${ }_{35-55} / \mathrm{IFA}$ treatment did not suppress EAE in C57BL/6J mice. 53

3.3.2.4 Adoptive transfer of $\mathrm{CD} 25^{+}$cells or crude DLN cells from

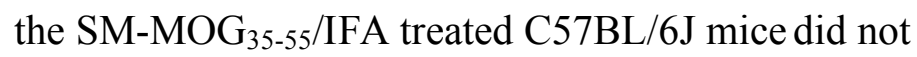
suppress EAE 54

3.3.3 Validation of the $\mathrm{SM}-\mathrm{MOG}_{35-55}$ treatment for EAE... 55

3.3.4 Identification of the SM targeting cells. 56

3.3.4.1 SM bound to $\mathrm{MHCII}^{+}$cells in vitro and in vivo 57

3.3.4.2 SM was captured by an $\mathrm{MHCII}^{-}$cell population in the blood

3.4 Discussion 62

3.5 Conclusions 64

Chapter 4: DM Binds to Blood Myeloid-Derived Suppressor Cells 65

4.1 Introduction 66

4.2 Aims 67

4.3 Results. 68

4.3.1 The MHCII binding capacity of DM is abrogated .68

4.3.1.1 $\mathrm{MHCII}^{+}$cells do not stain with DM in vitro or in vivo..... 68

4.3.1.2 Conjugation of DM to peptide did not enhance the peptide presentation to $\mathrm{CD} 4+$ cells 71

4.3.2 $\mathrm{DM}$ bound to the $\mathrm{MHCII}^{-} \mathrm{CD} 11 \mathrm{~b}^{+} \mathrm{F} 4 / 80^{+} \mathrm{Gr}-1^{+}$blood cells in vivo 72

4.3.3 The binding to the blood MHCII- cells was not specific to superantigen 74

4.3.4 The $\mathrm{DM}^{+}$cells were myeloid cells 75

4.3.5 The blood $\mathrm{MHCII} C D 11 \mathrm{~b}^{+} \mathrm{F} 4 / 80^{+} \mathrm{Gr}-1^{+}$cells were potent suppressors of $\mathrm{T}$ cell response 
4.3.5.1 $\mathrm{DM}^{+}$bound to the $\mathrm{CD} 11 \mathrm{~b}^{+} \mathrm{Ly} 6 \mathrm{G}^{-} \mathrm{SSC}^{\text {low }}$ cells in the blood ....... 78

4.3.5.2 $\mathrm{CD} 11 \mathrm{~b}^{+} \mathrm{Ly}_{6 \mathrm{G}}{ }^{-}$cell purification from the blood........................ 79

4.3.5.3. Validation of a suppression assay for measuring the blood

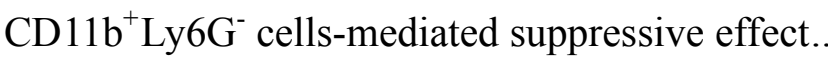

4.3.5.4 Naïve blood CD11 ${ }^{+}$Ly6G $^{-}$cells suppressed T cell

proliferation and thus express MDSC phenotype...... 84

4.3.6 Mechanisms for bMDSC-mediated suppression of $\mathrm{CD}^{+} \mathrm{T}$ cell proliferation. 86

4.3.6.1 $\mathrm{CD}^{+}$cells were direct targets of the bMDSC-mediated suppression.....

4.3.6.2 bMDSCs suppressed $\mathrm{CD}^{+}{ }^{+} \mathrm{T}$ cell proliferation in a cell-contact dependant manner.....

4.3.6.3 NOS but not arginase 1 was involved in the bMDSCs' suppression of $\mathrm{T}$ cell proliferation 88

4.3.6.4 The role of IFN- $\gamma$ in the bMDSC-mediated suppression of $\mathrm{T}$ cell proliferation

4.3.6.5 bMDSCs' suppression of T cell proliferation was not dependent on IDO.

4.3.6.6 The bMDSCs did not enhance responder cell apoptosis. 91

4.3.6.7 The bMDSC-mediated suppression of $\mathrm{T}$ cell proliferation was not dependent on Tregs. 92

4.3.7 Binding of DM with bMDSCs in vivo enhanced the bMDSCs' suppression of $\mathrm{T}$ cell proliferation in vitro 93

4.3.8 DM did not bind to bMDSCs in vitro..... 94

4.4 Discussion 95

4.5 Conclusions .98

Chapter 5: DM-MOG ${ }_{35-55}$ Suppresses EAE in a MOG $_{35-55}$ Specific Manner . 99

5.1 Introduction 100

5.2 Aims 100

5.3 Results. 101

5.3.1 DM-MOG $35-55$ suppressed $\mathrm{MOG}_{35-55}$ induced EAE in C57BL/6J mice .. 101

5.3.2 DM-MOG $35-55$ treatment reduced cell infiltration into the CNS . 103

5.3.3 The $\mathrm{MOG}_{35-55}$ peptide conjugated to DM was necessary for EAE suppression. 
5.3.4 DM-MOG ${ }_{35-55}$ mediated suppression of EAE was $\mathrm{MOG}_{35-55}$ specific .... 104

5.3.4.1 DM-MOG $\mathrm{DO}_{35-55}$ treatment specifically reduced DLN cells'

proliferation in response to $\mathrm{MOG}_{35-55}$ restimulation in vitro

5.3.4.2 DM-MOG $\mathrm{DO}_{35-55}$ treatment reduced DLN cells' production of IL-17 and IFN- $\gamma$ in response to $\mathrm{MOG}_{35-55}$ restimulation in vitro

5.3.5 bMDSCs played a key role in $\mathrm{DM}-\mathrm{MOG}_{35-55}$-mediated suppression

of EAE

5.3.5.1 Adoptive transfer of the bMDSCs from $\mathrm{DM}-\mathrm{MOG}_{35-55}$ treated mice suppressed EAE

5.3.5.2 bMDSCs depletion methods.

5.3.5.3 DM-MOG $35-55$ was more potent than $\mathrm{SM}^{-\mathrm{MOG}_{35-55} \text { to }}$ suppress EAE

5.3.5.4 DM-MOG $\mathrm{DO}_{35-55}$ treatment restored bMDSCs' in vitro suppressive capability

5.3.6 Optimisation of $\mathrm{DM}^{-\mathrm{MOG}_{35-55}}$ treatment delivery

5.3.6.1 i.v. injection of DM-MOG $35-55$ did not suppress EAE 114

5.3.6.2 s.c. injection of $\mathrm{DM}^{-\mathrm{MOG}_{35-55}}$ in CFA or IFA on the neck protected mice against EAE

5.3.6.3 s.c. injection of $\mathrm{DM}-\mathrm{MOG}_{35-55}$ in $\mathrm{PBS}$ on the neck protected mice against EAE.

5.3.7 i.v. injection of DM did not suppress EAE

5.4 Discussion

5.5 Conclusions

Chapter 6: General Discussion.

6.1 Overview

6.2 Clinical applications

6.3 Implications for future research

References

Appendix 1: Antibodies Used for Flow Cytometry

Appendix 2: Validation of DM-MOG D5-55 $_{3}$ Treatment for EAE 
1 Common buffers........................................................................................... 158

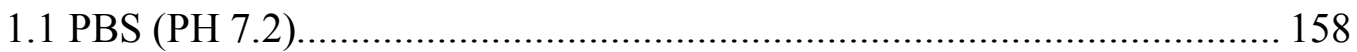

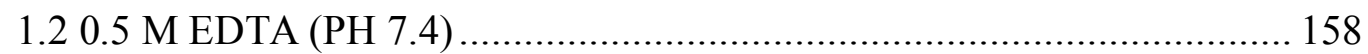

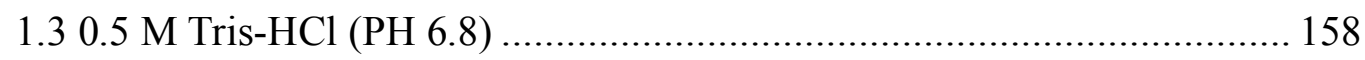

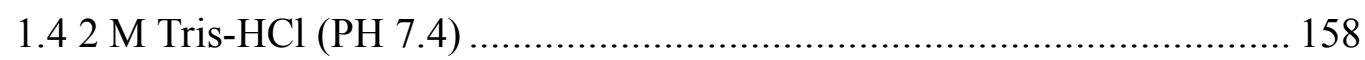

2 Buffers for tissue processing ................................................................................ 158

2.1 Alsevers solution for blood processing ............................................. 158

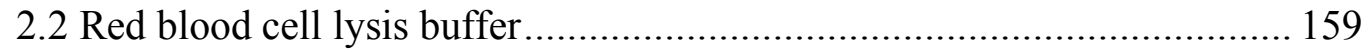

$2.31 .8 \% \mathrm{NaCl}$ buffer for red blood cell lyse ............................................... 159

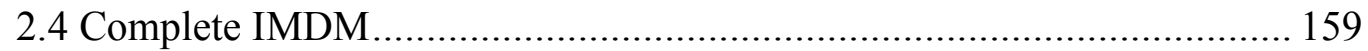

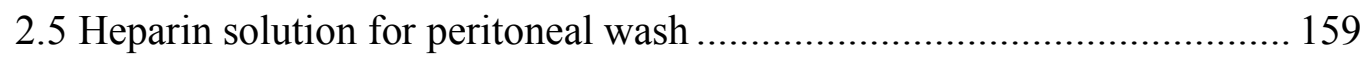

2.6 Hanks solution for cell processing .................................................... 159

2.7 Cell freezing medium ............................................................................ 160

2.8 Percoll diluent for cell isolation from spinal cord................................... 160

3 Buffers for FACS staining............................................................................... 160

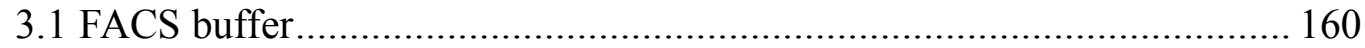

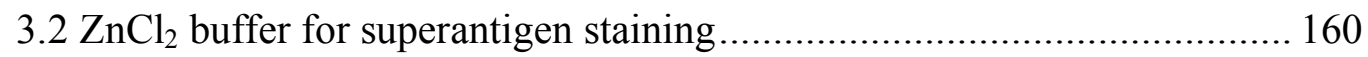

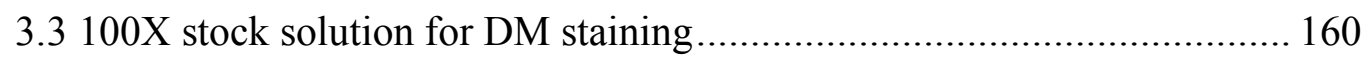

3.4 Fixation/Permeabilization buffer for Foxp3 intracellular staining ........... 161

3.5 Permeabilization buffer for Foxp3 intracellular staining ....................... 161

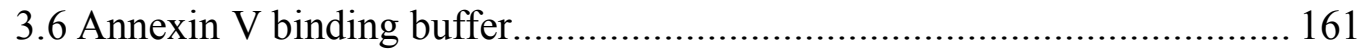

4 Buffers for superantigen coupling .......................................................................... 161

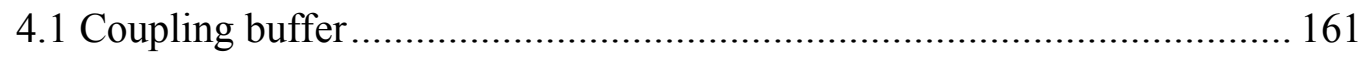

$4.220 \mathrm{mM}$ sodium phosphate buffer for superantigen storage ..................... 162

$4.30 .1 \%$ Acetic acid for peptide storage .................................................. 162

5 Buffers for NuPAGE ${ }^{\circledR}$ gel............................................................................. 162

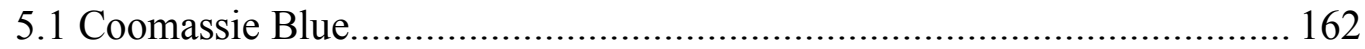

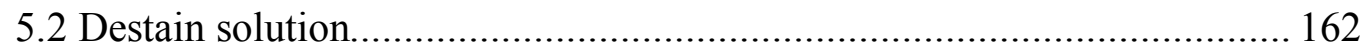

6 Buffers and solutions for injection............................................................. 162

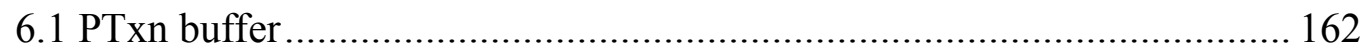

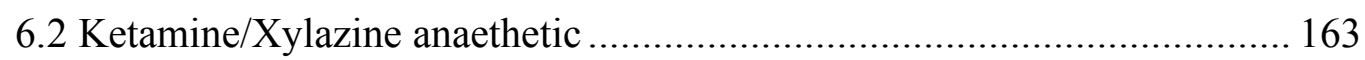

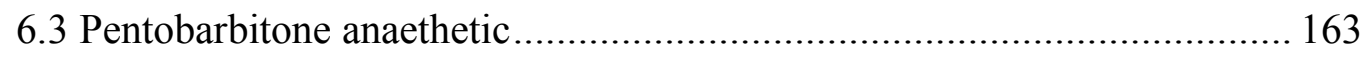

7 Buffers for cell separation .................................................................................... 163

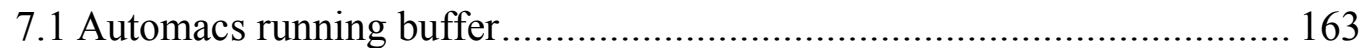


7.2 Automacs rinse buffer $x$ xii

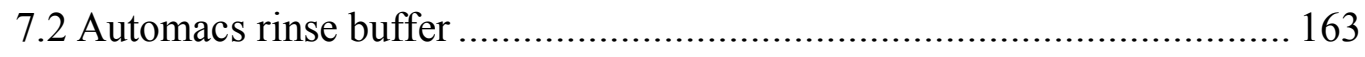

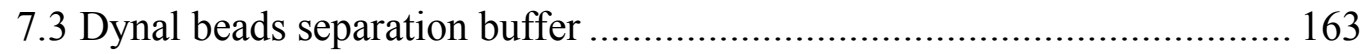

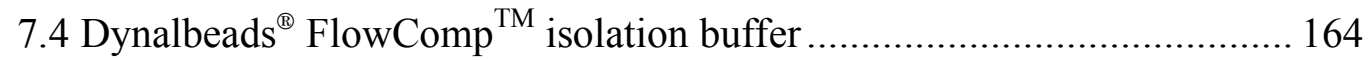

8 Buffers for protein labelling..................................................................... 164

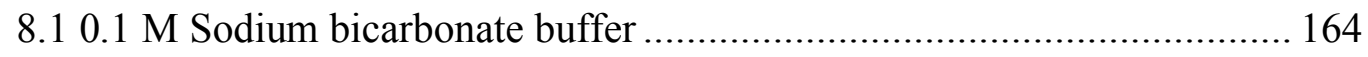

9 Buffers for protein concentration detection ...................................................... 164

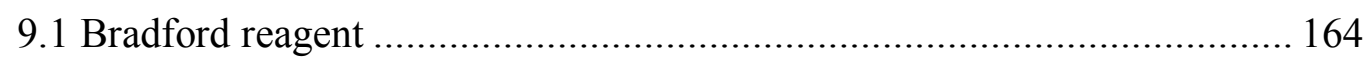

10 Buffers for cytokine detection ................................................................. 164

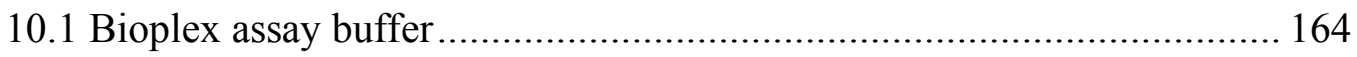

11 Solutions for Griess reaction ................................................................. 165

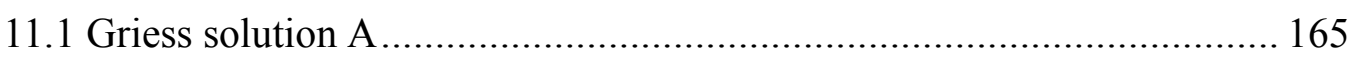

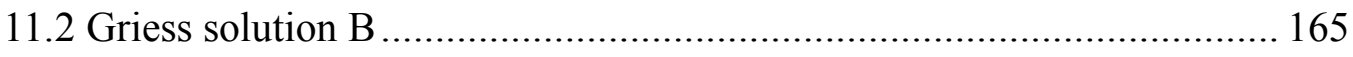

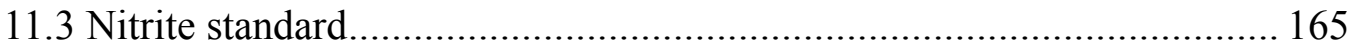




\section{List of Figures}

Figure 1.1 Differentiation of $\mathrm{CD}^{+} \mathrm{T}$ cell lineages

Figure 3.1 The $\mathrm{MOG}_{35-55 \mathrm{c}}$ and $\mathrm{OVA}_{323-339 \mathrm{c}}$ peptides could be efficiently conjugated to SM.....

Figure 3.2 The peptides conjugated to SM were efficiently presented to $\mathrm{CD}^{+} \mathrm{T}$ cells in vitro 50

Figure 3.3 The peptides conjugated to SM were efficiently presented to $\mathrm{CD} 4^{+} \mathrm{T}$ cells in vivo.

Figure 3.4 Adoptive transfer of the Foxp $3^{+}$cells from $\mathrm{SM}_{-\mathrm{MOG}_{35-55}} / \mathrm{IFA}$ treated mice did not suppress EAE in the recipient mice. 52

Figure 3.5 SM-MOG $35-55 /$ IFA treatment did not suppress EAE in Foxp3-GFP KI mice 53

Figure 3.6 SM-MOG $35-55 /$ IFA treatment did not suppress EAE in C57BL/6J mice 54

Figure 3.7 Adoptive transfer of neither CD25 ${ }^{+}$cells nor crude DLN cells from

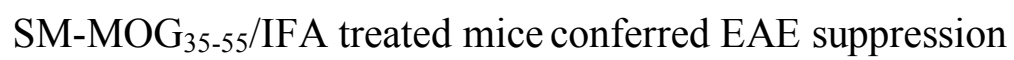
55

Figure 3.8 The addition of $\mathrm{SM}-\mathrm{MOG}_{35-55}$ to the EAE inducing emulsion caused a slight suppression of EAE 56

Figure 3.9 SM-Alexa 488 was captured by the $\mathrm{MHCII}^{+}$Thy $1.2^{-}$splenocytes in vitro 57

Figure 3.10 SM was captured by $\mathrm{MHCII}^{+}$cells in vivo.... 58

Figure 3.11 SM targeted all the major $\mathrm{MHCII}^{+}$cells in the spleen. 59

Figure 3.12 SM binding to DCs did not change the cells' maturation markers. 60

Figure 3.13 A blood-borne $\mathrm{MHCII}^{-}$cell population captured SM-Alexa 488. 61

Figure $3.14 \mathrm{SM}^{+} \mathrm{MHCII}^{-}$cells in the blood express F4/80, Gr-1 and $\mathrm{CD} 11 \mathrm{~b}$ 62

Figure 4.1 $\mathrm{MHCII}^{+}$cells did not stain with DM both in vitro and in vivo. 70

Figure 4.2 The peptide conjugated to DM and SM were differently presented to $\mathrm{CD}^{+} \mathrm{T}$ cells in vitro 72

Figure 4.3 A blood-borne cell population stained with SM- and DM-Alexa 488 ........ 74

Figure 4.4 The binding to the blood $\mathrm{MHCII}^{-}$cells was not specific to superantigen..... 75 Figure 4.5 DM was taken into the cytoplasm of a myeloid cell population in the blood. 77

Figure 4.6 The $\mathrm{DM}^{+}$cells were $\mathrm{CD} 11 \mathrm{~b}^{+} \mathrm{Ly}_{6} \mathrm{G}^{-}$ 78 
Figure 4.7 The CD11 ${ }^{+}{\text {Ly } 6 G^{-}}^{-}$cells had a low side scatter intensity profile

Figure 4.8 Two-step purification of $\mathrm{CD} 11 \mathrm{~b}^{+} \mathrm{Ly} 6 \mathrm{G}^{-}$cells from the blood ..................... 80

Figure 4.9 Purification of the $\mathrm{DM}^{+}$cells via the two-step purification procedure. 80

Figure 4.10 Comparison of thymidine incorporation and CFSE dilution based suppression assays

Figure 4.11 The blood CD11 ${ }^{+} \mathrm{Ly}_{6 \mathrm{G}^{-}}$cells mediated suppression of $\mathrm{CD} 4^{+} \mathrm{T}$ cells was temporary. 84

Figure 4.12 The blood CD11 ${ }^{+}$Ly6G $^{-}$cells suppressed an antigen-non-specific T cell response 85

Figure 4.13 Blood CD11 ${ }^{+} \mathrm{Ly}_{6 \mathrm{G}^{-}}$cells suppressed an antigen-specific response 85

Figure 4.14 The blood CD11 $\mathrm{b}^{+} \mathrm{Ly}_{6 \mathrm{G}^{-}}$cells suppressed the plate-bound anti-CD3 and anti-CD28 induced $\mathrm{T}$ cell response.....

Figure 4.15 bMDSCs suppressed the proliferation of purified $\mathrm{CD}^{+} \mathrm{T}$ cells 87

Figure 4.16 The bMDSC-mediated suppression was cell contact dependent 88

Figure 4.17 The bMDSCs' suppression of T cell proliferation was mediated by NOS but not arginase 1

Figure 4.18 The involvement of IFN- $\gamma$ in the bMDSCs' suppression of T cell proliferation.

Figure 4.19 IDO was not involved in the bMDSC-mediated suppression of T cell proliferation 91

Figure 4.20 The addition of the bMDSCs did not enhance the splenocyte apoptosis.....

Figure $4.21 \mathrm{bMDSCs}-$ mediated suppression of $\mathrm{CD}^{+} \mathrm{T}$ cell proliferation did not depend on Tregs.

Figure 4.22 The binding of DM with bMDSCs in vivo enhanced the bMDSC-mediated suppression of $\mathrm{T}$ cell proliferation in vitro. 94

Figure 5.1 DM-MOG $35-55$ suppressed EAE in a dose-dependent manner. 102

Figure 5.2 DM-MOG $35-55$ treatment reduced cell infiltration into the CNS 103

Figure 5.3 $\mathrm{MOG}_{35-55}$ peptide conjugated to DM was necessary for EAE suppression 104

Figure 5.4 DM-MOG $35-55$ treatment specifically reduced DLN cells' proliferation in response to $\mathrm{MOG}_{35-55}$ restimulation in vitro... 105

Figure 5.5 DM-MOG $35-55$ treatment reduced the DLN cells' production of IL-17 and IFN- $\gamma$ in response to $\mathrm{MOG}_{35-55}$ restimulation in vitro 
Figure 5.6 DM-MOG $35-55$ treatment did not alter IL-10, IL-4 or TNF- $\alpha$ production by DLN cells in response to the $\mathrm{MOG}_{35-55}$ restimulation in vitro

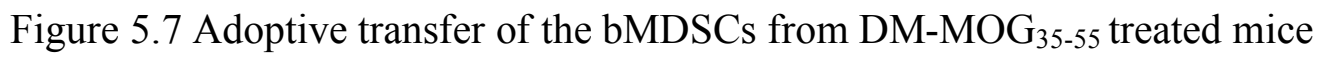
suppressed EAE

Figure 5.8 Depletion of the bMDSCs by i.v. injection of clodronate liposomes.

Figure 5.9 The administration of clodronate liposomes diminished 2D2 CD4 ${ }^{+}$cell proliferation to $\mathrm{MOG}_{35-55}$ in vivo .....

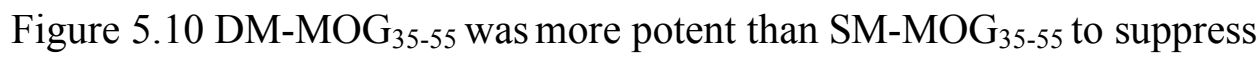
$\mathrm{MOG}_{35-55}$ induced $\mathrm{EAE}$ in $\mathrm{C} 57 \mathrm{BL} / 6 \mathrm{~J}$ mice

Figure 5.11 DM-MOG $35-55$ treatment restored the bMDSCs' in vitro suppressive capability

Figure 5.12 i.v. injection of DM-MOG ${ }_{35-55}$ did not suppress EAE 115

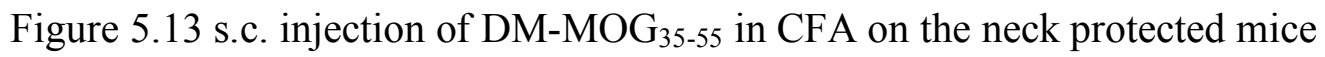
against EAE

Figure 5.14 s.c. injection of $\mathrm{DM}^{-\mathrm{MOG}_{35-55}}$ in IFA on the neck protected mice against EAE

Figure 5.15 Multiple s.c. injection of DM-MOG $\mathrm{DO}_{35-55}$ in PBS suppressed EAE

Figure 5.16 i.v. injection of DM did not suppress EAE

Figure 5.17 Hypothesised mechanisms for DM-MOG $35-55$ and bMDSCs mediated suppression of EAE 


\section{List of Tables}

Table 3.1 Optimisation of the SM-peptide conjugation .......................................... 48

Table 3.2 Variables for the optimisation of the $\mathrm{SM}-\mathrm{MOG}_{35-55}$ treatment for EAE........ 56

Table 4.1 The surface staining of $\mathrm{DM}^{+} \mathrm{CD} 1 \mathrm{~b}^{+}$cells in the blood............................... 76

Table 4.2 Methods utilised to investigate binding of DM-Alexa 488 to bMDSCs

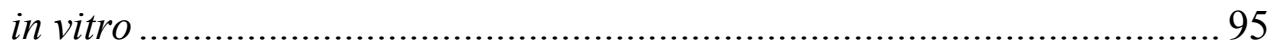

Table 5.1 Reproducibility of the treatment using DM-MOG $35-55$................................ 102

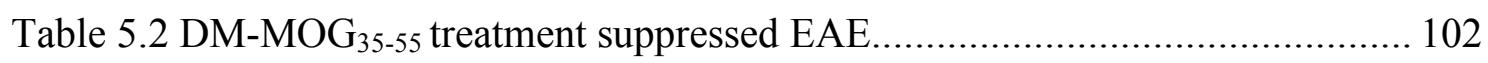




\section{Abbreviations}

\begin{tabular}{|c|c|}
\hline$\alpha \mathrm{GalCer}$ & $\alpha$-galactosyl ceramide \\
\hline $\mathrm{APC}$ & Antigen presenting cell \\
\hline BBB & Blood-brain barrier \\
\hline BMDC & Bone marrow-derived dendritic cell \\
\hline BSA & Bovine serum albumin \\
\hline $\mathrm{C}$ & Cysteine \\
\hline CD & Cluster of differentiation \\
\hline CFA & Complete Freund's adjuvant \\
\hline CFSE & Carboxy-fluorescein diacetate, succinimidyl ester \\
\hline CNS & Central nervous system \\
\hline $\mathrm{CO}$ & Cut off \\
\hline $\mathrm{CO}_{2}$ & Carbon dioxide \\
\hline ConA & Concanavalin A \\
\hline CPM & Counts per minute \\
\hline CTLA-4 & Cytotoxic T lymphocyte associated antigen 4 \\
\hline DAPI & 4',6-diamidino-2-phenylindole \\
\hline $\mathrm{DC}$ & Dendritic cell \\
\hline $\mathrm{DH}_{2} \mathrm{O}$ & Distilled water \\
\hline DM & Double mutant \\
\hline DMSO & Dimethyl sulphoxide \\
\hline DTH & Delayed-type hypersensitivity \\
\hline DTT & Dithiothreitol \\
\hline EAE & Experimental autoimmune encephalomyelitis \\
\hline EDTA & Ethylenediaminetetraacetic acid \\
\hline FACS & Fluorescence activated cell sorting \\
\hline FITC & Fluorescein isothiocyanate \\
\hline FCS & Fetal calf serum \\
\hline Foxp3 & Forkhead box P3 \\
\hline FSC & Forward scatter \\
\hline GA & Glatiramer acetate \\
\hline GFP & Green fluorescent protein \\
\hline GITR & $\begin{array}{l}\text { Glucocorticoid-induced tumour necrosis factor receptor family } \\
\text { related receptor }\end{array}$ \\
\hline GM-CSF & Granulocyte-macrophage colony-stimulating factor \\
\hline $\mathrm{H} \& \mathrm{E}$ & Hematoxylin and eosin \\
\hline HEPES & 4-(2-hydroxyethyl)-1-piperazineethanesulfonic acid \\
\hline HLA & Human leukocyte antigen \\
\hline HyNic & 6-HydrazinoNicotinamide \\
\hline ICAM-1 & Intracellular adhesion molecule-1 \\
\hline IDO & Indoleamine-2,3-dioxygenase \\
\hline IFA & Incomplete Freund's adjuvant \\
\hline IFN $\gamma$ & Interferon gamma \\
\hline IL & Interleukin \\
\hline i.p. & Intraperitoenal \\
\hline IMDM & Iscoves modified Dulbecco's medium \\
\hline i.v. & Intravenous \\
\hline $\mathrm{KDa}$ & Kilo Dalton \\
\hline KI & Knock-in \\
\hline
\end{tabular}




\begin{tabular}{|c|c|}
\hline L-NMMA & $\mathrm{N}^{\mathrm{G}}$-monomethyl-L-arginine \\
\hline LPS & Lipopolysaccharide \\
\hline MAG & Myelin associated glycoprotein \\
\hline MBP & Myelin basic protein \\
\hline MDSC & Myeloid-derived suppressor cell \\
\hline MHC & Major histocompatibility complex \\
\hline MS & Multiple sclerosis \\
\hline MW & Molecular weight \\
\hline mSAg & Modified superantigen \\
\hline MOG & Myelin oligodendrocyte glycoprotein \\
\hline NKT & Natural killer T cell \\
\hline NOD & Non-obese diabetic \\
\hline NOS & Nitric oxide synthase \\
\hline nTreg & Naturally arising regulatory $\mathrm{T}$ cell \\
\hline OVA & Ovalbumin \\
\hline Pam3Cys & Pam3Cys-SKKKK \\
\hline PBS & Phosphate buffered saline \\
\hline $\mathrm{PE}$ & Phycoerythrin \\
\hline PerCP & Peridinin chlorophyll A protein \\
\hline PI & Propidium iodide \\
\hline PLP & Proteolipoprotein \\
\hline PMA & Phorbol 12-myristate 13-acetate \\
\hline PPD & Mycobacterium-derived purified protein derivative \\
\hline PTxn & Pertussis toxin \\
\hline rpm & Revolutions per minute \\
\hline s.c. & Subcutaneous \\
\hline SDS PAGE & Sodium dodecyl sulfate polyacrylamide gel electrophoresis \\
\hline SEM & Standard error of mean \\
\hline SM & Single mutant \\
\hline SMEZ & Streptococcal mitogenic exotoxin Z \\
\hline SPE-C & Streptococcal pyrogenic exotoxin C \\
\hline SSC & Side scatter \\
\hline TCR & T cell receptor \\
\hline TGF- $\beta$ & Transforming growth factor-beta \\
\hline Th17 & IL-17 producing T cells \\
\hline TLR & Toll-like receptor \\
\hline TNF- $\alpha$ & Tumour necrosis factor-alpha \\
\hline Treg & Regulatory $\mathrm{T}$ cell \\
\hline $\mathrm{v} / \mathrm{v}$ & Volume/volume \\
\hline $\mathrm{w} / \mathrm{v}$ & Weight/volume \\
\hline
\end{tabular}

$\mathrm{N}^{\mathrm{G}}$-monomethyl-L-arginine

Myelin associated glycoprotein

Myelin basic protein

Myeloid-derived suppressor cell

Major histocompatibility complex

Multiple sclerosis

Modified superantigen

Myelin oligodendrocyte glycoprotein

Natural killer T cell

Non-obese diabetic

Naturally arising regulatory $\mathrm{T}$ cell

bumin

Phosphate buffered saline

Phycoerythrin

Propidium iodide

Proteolipoprotein

Phorbol 12-myristate 13-acetate

Mycobacterium-derived purified protein derivative

Pertussis toxin

Standard error of mean

Single mutant

Streptococcal mitogenic exotoxin Z

Streptococcal pyrogenic exotoxin $\mathrm{C}$

Side scatter

T cell receptor

Toll-like receptor

tumour necrosis factor-alpha

Weight/volume 


\section{Chapter 1:}

\section{General Introduction}


The immune system is composed of two major arms, the innate and adaptive immune systems, whose principal function is to eliminate invading pathogens that may cause damage to the body. The innate immune system forms the first line of the body's defence against pathogens and responds rapidly to invading organisms, but cannot confer immunological memory to the host and is antigen non-specific. In contrast, the adaptive immune system reacts more slowly than the innate system, but allows for the generation of immunological memory to past infections and is antigen-specific. The specificity of the adaptive immune response requires diversity of the $\mathrm{T}$ cell repertoire, which enables it to recognise a broad range of pathogens. However, this diversity also increases the risk of developing autoimmunity, the condition in which the immune system reacts against the body's own cells. To maintain tolerance to self, the immune system utilises multiple regulatory mechanisms including central tolerance and peripheral tolerance (see Section 1.2.1 and Section 1.2.2). Autoimmunity results when one or more of these tolerance mechanisms fail ${ }^{1-3}$.

The general introduction summarises the two arms of the immune system (the innate and adaptive systems) and tolerance mechanisms that prevent autoimmunity, it discusses the cause and potential treatments for multiple sclerosis (MS), an autoimmune disease of the central nervous system (CNS), and describes the mouse model used to study MS, experimental autoimmune encephalomyelitis (EAE).

\subsection{The mammalian immune system}

The mammalian immune system is comprised of a complex network of specialised cells and soluble mediators that have evolved to protect the host against pathogens. Two fundamentally different types of immune responses are present in most mammals - the innate and adaptive immune responses ${ }^{1,3-5}$. The classification of the cells as innate or adaptive is primarily based on the nature of the receptors they express. Innate receptors are encoded in the genome as separate genes, while adaptive receptors are encoded as arrays of gene segments that are randomly recombined into diverse receptors ${ }^{1,3}$. The innate and adaptive immune systems have evolved interactively in concordance with mammalian history and environment ${ }^{5}$ and the interactions between them are essential for effective immune function ${ }^{1,3,4}$. 


\subsubsection{The innate immune system}

A key role of the innate immune system is to provide the first line of defence against invading pathogens. As such, innate immune responses are rapid to ensure that the invading pathogens are detected and destroyed within minutes to hours after infection ${ }^{2}$. The components of the innate immune system include anatomical barriers (e.g. skin and mucosal membranes), soluble proteins (e.g. complement), phagocytic cells that scavenge extracellular macromolecules, and inflammation ${ }^{2,6}$.

An important part of active defence in the innate immune system is the detection of pathogen-associated molecular patterns (PAMPs), the molecular motifs commonly found in bacteria, fungi, parasites and other micro-organisms. This process is mediated by the germline-encoded receptors on innate immune cells that are termed "pattern recognition receptors" (PRRs). The PRRs have evolved to recognise the PAMPs and have minimal reactivity with self-cells. For example, many of these PRRs recognise unique carbohydrate structures associated with the cell walls of bacteria and yeasts that are essential for the survival of the micro-organisms ${ }^{2}$.

\subsubsection{Toll like receptors}

Toll-like receptors (TLRs) are part of the family of PPRs and have been identified in insects, plants and mammals ${ }^{6}$. Currently, there are at least 13 TLRs described in mice and 11 in humans. Each TLR binds to a variety of PAMPs and the specificity of TLRs' has been well defined except TLR10, 12 and 13. For example, TLR3 binds to virus-derived double-stranded ribonucleic acid (RNA), TLR4 interacts with lipopolysaccharide (LPS) that is a part of the outer membrane of Gram-negative bacteria, and TLR9 binds specifically to unmethylated CpG motifs characteristically found in bacterial deoxyribonucleic acid (DNA) ${ }^{7}$.

The binding of TLRs to their ligands triggers two intracellular signalling pathways, which ultimately activate NF- $\kappa B$ in the nucleus. With the exception of TLR3, TLRs activate cells through the myeloid differentiation primary response gene 88 (MyD88)-dependent pathway ${ }^{7}$. In contrast, TLR3 signals in a MyD88-independent fashion via interferon (IFN) regulatory factor-3 and other IFN-responsive factors. Notably, one TLR, TLR4, signals through both pathways ${ }^{7,8}$. Following the binding of TLRs to their ligands, several reactions can possibly occur. Signalling through TLRs 
can lead to the synthesis and secretion of pro-inflammatory cytokines and lipid mediators, thereby initiating inflammatory responses and inducing appropriate responses against pathogens. Alternatively, TLR stimulation can result in the activation of antigen presenting cells (APCs) and thereby initiate adaptive immune responses ${ }^{2,6-8}$. Therefore, TLR signalling provides an important link between the innate and adaptive immune systems and plays a key role in the effective activation of the antigen-specific adaptive immune responses ${ }^{2}$.

\subsubsection{Cells of the innate immune system}

Cells of the innate immune system include mast cells, natural killer cells, eosinophils, basophils and phagocytic cells. Most innate immune cells cannot divide in the periphery, but are produced by pluripotent hematopoietic stem cells in the bone marrow ${ }^{9}$.

Phagocytic cells, including monocytes, macrophages, neutrophils and dendritic cells (DCs), are the major effector cells in innate immune responses ${ }^{2,6}$. These cells express an array of PPRs on their surface, including TLRs, C-type lectin, ficolin, integrin $\mathrm{CD} 11 \mathrm{~b} / \mathrm{CD} 18$ and scavenger receptors. Macrophages (a type of tissue-associated phagocytes) and monocytes (a type of mononuclear phagocytes in the blood) are located at sites where they are likely to encounter pathogens. These phagocytes are the first line of defence against invading pathogens. Pathogen recognition by phagocytes results in both pathogen destruction and recruitment of additional phagocytes to the site of pathogen invasion. An important feature of phagocyte activation is the secretion of cytokines such as interleukin (IL)-1, IL-6, IL-8 and tumour necrosis factor alpha (TNF- $\alpha$ ), which are critical to the development and function of both the innate and adaptive immune responses. In addition to attacking pathogens directly, some phagocytes, such as macrophages and dendritic cells (DCs), also act as APCs and induce adaptive immune responses ${ }^{6}$.

\subsubsection{The adaptive immune system}

The adaptive immune system is the second line of the body's defence and reacts more slowly than the innate immune system in response to invading pathogens. However, the 
adaptive immune system possesses two advantages over the innate immune system: specificity and memory. These two features allow the immune system to recognise and remember specific pathogens and mount stronger attacks for the second encounter of the same pathogen ${ }^{3}$.

Specificity refers to the fact that each adaptive immune cell recognises only one particular antigen. This property is achieved by the generation of a diverse array of cell surface recognition receptors by the processes called V(D)J recombination (a genetic recombination of antigen receptor gene segments) and somatic hypermutation (a process of accelerated somatic mutations). These mechanisms allow a small number of genes to generate a vast pool of different antigen receptors, such as T-cell receptors (TCRs) and B-cell receptors (BCRs). After maturation, each individual adaptive immune cell displays only one receptor from this pool, providing it with a unique specificity $^{3}$.

Memory is defined as the event where previously activated lymphocytes re-encounter an antigen and develop a greater and faster response than the first encounter. The primary response to an antigen starts with the naïve lymphocytes that have not previously encountered antigens. Upon initial pathogen exposure, these cells expand in numbers, become effector cells and then a proportion of these lymphocytes persist in a resting state (i.e. memory cells), where they remain ready to initiate a secondary response upon re-encounter with this antigen. When the same pathogen attacks the immune system for the second time, memory cells developed during primary response rapidly proliferate and react against this antigen, which results in a faster and stronger response (i.e. secondary response) ${ }^{10}$.

\subsubsection{Antigen presenting cells}

Antigen presenting cells (APCs) are cells that are able to take up and destroy pathogens. Subsequently, they can present the pathogenic antigens on their surface via the major histocompatibility complex (MHC) molecules to T cells, which leads to the initiation of an adaptive immune response. APCs are divided into two categories-professional APCs and non-professional APCs. The APCs that are specialised to initiate or promote lymphocyte activation are termed as "professional APCs". Professional APCs include DCs, macrophages and B cells. These cells express MHC class II (MHCII) molecules 
and have various specialised characteristics, such as the expression of co-stimulatory molecules to modulate the activation of responding lymphocytes ${ }^{11}$. In contrast, the other cells do not constitutively express MHCII molecules on their surface but can be stimulated to present antigens, and are termed as "non-professional APCs". Non-professional APCs include fibroblasts, thymic epithelial cells, thyroid epithelial cells, glial cells, pancreatic beta cells and vascular endothelial cells ${ }^{12}$.

DCs are the major professional APCs involved in initiating T cell responses ${ }^{11}$. DCs capture and process antigens at the site of exposure in peripheral tissues. Then DCs mature and migrate to lymphoid tissues where they present antigens and activate naïve lymphocytes in an antigen-specific manner. The induction of an adaptive immune response is dependent on the phenotype of the DCs that present antigens, including the cytokines they produce and the co-stimulatory molecules they express ${ }^{11}$.

\subsubsection{T cells}

$\mathrm{T}$ lymphocytes ( $\mathrm{T}$ cells) are thymus-derived and are key cellular players in adaptive immunity. T cells express TCRs, which recognise specific antigens bound to MHC class I or II molecules. T cells that express the co-receptor CD8 are cytotoxic T lymphocytes (CTLs) and recognise peptides presented on MHCI, whereas T cells that express CD4 ( $\mathrm{T}$ helper cells (Th cells)) recognise peptides presented on MHCII molecules $^{13}$.

The main function of $\mathrm{CD}^{+} \mathrm{CTLs}$ is to kill infected or transformed cells ${ }^{13}$. There are two mechanisms utilised by CTLs for killing target cells. First, CTLs can release the cytotoxins perforin and granzymes. Perforin forms pores in the target cell's plasma membrane allowing granzymes to enter the target cells and these enzymes trigger target cell apoptosis. The second way to kill target cells is via ligation of FAS by FAS ligand (FasL). The binding of FasL on CTLs to Fas molecules on target cells leads to caspase-mediated apoptosis of the target cells ${ }^{14}$.

The principal function of $\mathrm{CD}^{+}$Th cells is to direct immune responses. $\mathrm{CD} 4^{+}$Th cells control B-cell antibody class switching, support antibody production, promote CTLs proliferation, differentiation and activation, and increase macrophages' bactericidal activity. The activation of $\mathrm{CD}^{+}$Th cells requires recognition of cognate 
peptide-MHCII complexes on professional APCs as well as co-stimulation through triggering CD28 on $\mathrm{CD}^{+}$Th cells by CD80/CD86 on APCs (see Section 1.1.3.4) ${ }^{13}$. Once activated, $\mathrm{CD} 4^{+}$Th cells can differentiate into Th1, Th2 or Th17 cells according to their cytokine profile ${ }^{15}$. The differentiation of $\mathrm{CD}^{+}$Th cells will be discussed in detail in Section 1.7.1.

\subsubsection{MHC-TCR interaction}

\subsubsection{MHC}

MHC is located on chromosome 6 in humans and chromosome 17 in mice. In humans, MHC genes are also called human leukocyte antigen (HLA) genes, whereas in mice, they are also known as H-2 genes. These genes are polygenic (multiple genes) and polymorphic (multiple alleles). The function of $\mathrm{MHC}$ is to bind and display peptides for appropriate $\mathrm{T}$ cell recognition. It is believed the polygenic and polymorphic nature of MHC increase the ability of APCs to present a wide array of immunogenic peptides ${ }^{11,13}$.

There are two types of MHC molecules that are recognised by TCRs. MHC class I (MHCI) and MHCII. MHCI molecules are able to present cytosolic peptides to CD $8^{+}$ CTLs and are ubiquitously expressed on all nucleated cells ${ }^{16}$. The MHCI molecule is composed of a glycosylated heavy $\alpha$ chain, which is non-covalently linked to the non-MHC encoded $\beta 2$ microglobulin. Two of the $\alpha$ chain helices form the peptide-binding groove that can bind peptides of 8-10 amino acids ${ }^{16}$.

MHCII molecules are able to present peptides derived from proteins in the extracellular space to $\mathrm{CD}^{+} \mathrm{T}$ cells, and the constitutive expression of MHCII molecule is restricted to professional APCs, such as DCs, B cells and macrophages ${ }^{13}$. MHCII molecule is composed of a two-helix $\alpha$ chain and a two-helix $\beta$ chain. The two helices of the $\alpha$ chain are called $\alpha 1$ and $\alpha 2$ and the two helices of the $\beta$ chain are called $\beta 1$ and $\beta 2$. The $\alpha 1$ and $\beta 1$ chains contain polymorphic regions and interact to form the peptide-binding grooves, while the $\alpha 2$ and $\beta 2$ chains contain immunoglobulin domains and form the membrane proximal regions. MHCII molecule can present a wide range of peptide length to $\mathrm{CD}^{+} \mathrm{T}$ cells. MHCII binding peptides usually vary between 15-20 amino acids in length, although shorter or longer lengths have also been identified ${ }^{17,18}$. 


\subsubsection{TCRs}

T-cell receptors (TCRs) belong to the immunoglobulin superfamily and are composed of membrane bound $\alpha$ and $\beta$ chains. The $\alpha$ chain and $\beta$ chain of TCRs each contains a variable region for antigen recognition and a constant region for association with CD3 components. The variable region of TCRs is formed by splicing and recombination of $\mathrm{V}, \mathrm{D}$ and $\mathrm{J}$ genes during $\mathrm{T}$ cell development in the thymus. The most variable region of TCRs is the complementarily determining region (CDR) 3 domain. CDR3 interacts directly with the peptides bound to MHC molecules and is critical in peptide recognition ${ }^{19}$. CDR1 and CDR2 are encoded in the germ line and are therefore much less variable. CDR1 and CDR2 are important in determining whether the TCR interacts with a MHCI or II molecule ${ }^{13}$. The CD3 complexes that are noncovalently associated with the constant region of TCRs are important for the TCR signal transduction. In most $\mathrm{T}$ cells, the CD3 complex is made up of four distinct subunits, $\gamma, \delta, \varepsilon$ and $\zeta$ subunits. These subunits are organised into three dimeric transduction modules: $\gamma \varepsilon, \delta \varepsilon$ and $\zeta \zeta$, and associate with TCRs to generate a signalling cascade leading to T cell activation ${ }^{20}$.

\subsubsection{MHC-TCR interaction}

The activation of antigen-specific $\mathrm{T}$ cells requires their TCRs to bind and recognise both the antigen and the MHC molecule that presents the antigen. The requirement for $\mathrm{T}$ cells to recognise a peptide in the context of a particular $\mathrm{MHC}$ is called "MHC restriction", 13 .

When a $\mathrm{T}$ cell binds to a MHC-peptide complex, it sits in an orientation that allows the CDRs of the TCR to interact with both the peptide and MHC molecule ${ }^{21}$. To trigger the TCR signalling pathway, the peptide-MHC complex is not required to bind to TCRs with high affinity ${ }^{22}$. However, sufficient duration of the interaction between peptide-MHC complex and TCR is important for the initiation of $\mathrm{T}$ cell cycle progression and maintenance of gene transcription ${ }^{23}$. In fact, it is believed that in vivo, a few agonistic peptide-MHC complexes engage and trigger a much larger number of TCRs in a serial triggering process ${ }^{24,25}$. Therefore, the most effective peptide-MHC complexes are the ones that are able to engage a TCR for a short period, then dissociate from the TCR and become available for new interactions with other T cells bearing the same $\mathrm{TCR}^{24,25}$. 
A unique characteristic of $\mathrm{T}$ cell activation is that although the specificity of antigen recognition is provided by TCR interaction with peptide-MHC complex, the outcome of the interaction also depends on the integration of signals arising from the accessory molecules engaged at the cellular interface. These accessory molecules include cell adhesion molecules, co-stimulatory molecules (discussed in the next section) and co-receptors such as CD4 or $\mathrm{CD}^{26}$ that are expressed on T cells and bind to MHC molecules to enhance the interaction between TCRs and MHC molecules ${ }^{27}$.

\subsubsection{Co-stimulation}

Naïve $\mathrm{T}$ cells require two signals to be fully activated. In addition to the first signal provided through the interactions between TCRs and peptide-MHC complexes described above, a second signal, co-stimulatory signal, is also required. Co-stimulation is antigen non-specific and is provided by the interactions between co-stimulatory molecules on APCs with their ligands on T cells. The absence of co-stimulation during TCR crosslinking of a naïve $\mathrm{T}$ cell results in partial activation that promotes $\mathrm{T}$ cell apoptosis, deletion or anergy (a state of unresponsiveness) ${ }^{25,28}$.

The best-characterised co-stimulatory molecules are CD80 and CD86. CD80 is the first identified co-stimulatory ligand and binds to CD28 on T cells. The interaction of CD80 and CD28 provides a co-stimulatory signal necessary for $\mathrm{T}$ cell priming ${ }^{29}$. However, the finding that blocking CD80 signalling did not significantly inhibit $\mathrm{T}$ cell activation led to the discovery of a second co-stimulatory molecule, CD86, which has a similar structure and function to CD80. Both CD80 and CD86 are expressed on activated APCs and the functional differences between the two molecules are yet to be defined ${ }^{30}$.

A major receptor for CD80 and CD86 on T cells is CD28. The CD28 glycoprotein is expressed constitutively on all human $\mathrm{CD}^{+} \mathrm{T}$ cells, $\sim 50 \%$ human $\mathrm{CD} 8^{+} \mathrm{T}$ cells and all murine $\mathrm{T}$ cells. Stimulation through $\mathrm{CD} 28$ in addition to TCR provides a potent co-stimulatory signal to $\mathrm{T}$ cells for cell activation and production of cytokines ${ }^{31}$.

Cytotoxic T lymphocyte associated protein 4 (CTLA-4), also known as CD152, is an additional receptor for CD80 and CD86 expressed on T cells. CTLA-4 is not constitutively expressed, but is up-regulated in low copy numbers by $\mathrm{T}$ cells for a short time after $\mathrm{T}$ cell activation. The up-regulation of CTLA-4 depends upon the ligation of 
CD28. The binding of CTLA-4 to CD80/CD86 has a 20 -fold higher affinity than CD28 and the binding of CTLA-4 transmits an inhibitory signal to T cells. The time that CTLA-4 is active overlaps with the time that CD28 signalling is reduced. Thus, CTLA-4 acts to limit the extent of the immune responses or to prevent immune responses from occurring ${ }^{31}$.

\subsection{Immunological tolerance}

The adaptive immune system generates a vast diversity of different receptors by genetic recombination. This process produces many receptors capable of recognising a wide array of antigens including self-antigens. To avoid immune responses that target self, protective mechanisms are required to eliminate or down-regulate self recognition. The term immunological tolerance describes the mechanisms that the immune system uses to suppress an immune respons $\mathrm{e}^{32}$. Using these immunological tolerance mechanisms, the immune system is delicately balanced between pathogen-driven immunity and self-antigen driven tolerance.

The regulation of self-tolerance of $\mathrm{T}$ cell repertoire is at two levels. First, the development and selection of $\mathrm{T}$ cells in the thymus is strongly biased against self-activity (central tolerance) ${ }^{33,34}$. Second, mature $\mathrm{T}$ cells that escape from the thymus are subject to a variety of suppressive mechanisms in the periphery (peripheral tolerance $)^{35}$. These two forms of tolerance will be discussed in the following sections.

\subsubsection{Central tolerance}

Central tolerance is the primary mechanism responsible for elimination of autoreactive $\mathrm{T}$ cells. This occurs in the thymus where immature $\mathrm{T}$ cells have rearranged their antigen receptor genes and encounter peptides presented by thymic APCs. About $98 \%$ of the thymic $\mathrm{T}$ cells die during development by failing either positive selection or negative selection (discussed below) ${ }^{32}$.

Positive selection aims to select for T cells capable of interacting with MHC molecules. Failure of a TCR to interact with peptide-MHC ligands is interpreted as a lack of self-MHC restriction, which will result in no biological function in the periphery. These thymocytes undergo death by neglect. An estimated $90 \%-95 \%$ of apoptotic cell death in the thymus is due to positive selection. However, this process does not remove the $T$ 
cells that may cause autoimmunity. Instead, these potential autoreactive $\mathrm{T}$ cells are removed by negative selection ${ }^{32}$.

Negative selection aims to remove the $\mathrm{T}$ cells that have too high an affinity for self-peptides and thus are potentially autoreactive. These $\mathrm{T}$ cells are eliminated by clonal deletion. To facilitate the deletion of potentially autoreative $\mathrm{T}$ cells for tissue antigens, the stromal or bone marrow-derived thymic cells express a wide range of host tissue-restricted self-antigens. This phenomenon has been termed promiscuous gene expression. Except for the involvement of the autoimmune regulator (Aire), the regulation of this gene expression pattern is poorly understood ${ }^{32}$.

\subsubsection{Peripheral tolerance}

Central tolerance eliminates the repertoire of immature lymphocytes with potential self-reactivity, however, sometimes central tolerance fails. For example, when an antigen synthesised in peripheral non-lymphoid tissues is not presented in high quantity in the thymus, some autoreative lymphocytes may escape into the periphery. Peripheral tolerance is therefore necessary to control these autoreactive lymphocytes and prevent autoimmunity. Peripheral tolerance consists of a variety of mechanisms including anergy (discussed below) and immune suppression ${ }^{6,36}$.

One mechanism of peripheral tolerance is anergy, which describes a state in which lymphocytes are functionally unresponsive $\mathrm{e}^{36}$. In the case of $\mathrm{T}$ cells, the induction of anergy is dependent on the signals they receive. During the induction of an antigen-specific response, naïve $T$ cells are activated when they receive signals through their TCRs and a co-stimulatory molecule. In contrast, if a naïve T cell receives signals through the TCR in the absence of co-stimulation, anergy results ${ }^{13,28}$. The requirement for the simultaneous delivery of antigen-specific and co-stimulatory signals by the same APC in the activation of naïve T cells means that only the appropriately activated APCs can initiate $\mathrm{T}$-cell responses. This is important, because not all the autoreactive $\mathrm{T}$ cells are deleted in the thymus and self-tolerance could be broken if naïve autoreactive $T$ cells recognised self-antigens on tissue cells and are then co-stimulated by a different APC. Thus, the requirement that the same cell presents both antigen and co-stimulatory signal is important in preventing destructive immune responses to self-tissues. The anergy of T cells is an essential mechanism for immune tolerance ${ }^{37}$ and inducing anergy 
by antigen recognition in the absence of co-stimulation has been considered for therapeutic use to suppress autoimmunity ${ }^{38,39}$. For example, the anti-CD3 mAb, OKT3, that initiates the TCR signalling pathway in the absence of co-stimulatory activity has been used clinically for the treatment of organ transplantation rejection and various autoimmune diseases ${ }^{40}$. Trials of oral anti-CD3 are planned for $\mathrm{MS}^{41}$.

A second mechanism of peripheral tolerance is immune suppression, whereby immune cells exert immune suppressive effects on other cells. For example, CTLA-4 and programmed death 1 (PD-1) are expressed on the surface of $\mathrm{CD}^{+} \mathrm{T}$ cells and the binding to these molecules transmits inhibitory signals to $\mathrm{CD} 4^{+} \mathrm{T}$ cells ${ }^{42,43}$. Moreover, it is now widely recognised that the peripheral cells specialised for suppression play a crucial role in the maintenance of immune suppression ${ }^{44}$, for example, regulatory $\mathrm{T}$ cells (Tregs) ${ }^{44}$ and myeloid derived suppressor cells (MDSCs) ${ }^{45}$. As such, targeting of these cells has great therapeutic potential for treating autoimmunity.

\subsection{Regulatory $T$ cells}

The identification of regulatory $\mathrm{T}$ cells (Tregs) as an important component of self-tolerance has opened a primary area of investigation in immunology. There is accumulating evidence that Tregs suppress the activation and expansion of self-reactive $\mathrm{T}$ cells, thereby preventing autoimmune diseases ${ }^{44}$. There have been several different types of Tregs discovered so far, including naturally arising Foxp $3{ }^{+} \mathrm{CD} 4^{+} \mathrm{CD} 25^{+}$Tregs (nTregs), IL-10-secreting Tr1 cells, TGF- $\beta$-secreting Th3 cells, Qa-1-restricted CD8 ${ }^{+} \mathrm{T}$ cells, $\mathrm{CD}^{+} \mathrm{CD} 28^{-} \mathrm{T}$ cells, $\mathrm{CD} 8^{+} \mathrm{CD} 122^{+} \mathrm{T}$ cells and $\gamma / \delta \mathrm{T}$ cells ${ }^{44}$. Among these various Treg populations, nTregs have been most intensively studied in humans and mice.

\subsubsection{Naturally arising Foxp3 ${ }^{+}$Tregs}

nTregs are produced by the thymus as a distinct T-cell subpopulation and play an indispensable role in self-tolerance. They express TCRs skewed for self-antigen recognition, and thus are poised for the prevention of autoimmunity ${ }^{44}$. The identification of the cell surface marker CD25 on nTregs, the IL-2 receptor $\alpha$ (IL-2 $\alpha$ ), has greatly facilitated the study of $\mathrm{nTregs}^{46}$. However, conventional non-regulatory $\mathrm{T}$ cells up-regulate CD25 during immune activation ${ }^{47}$. Thus, distinct markers are required to differentiate nTregs from other types of $\mathrm{T}$ cells. Although a constellation of cell 
surface proteins, including CD $25^{46}, \mathrm{CTLA}-4^{48}, \mathrm{CD} 122^{49,50}, \mathrm{Nrp}^{51}, \mathrm{CD} 5^{52}, \mathrm{CD} 103^{53,54}$, $\mathrm{CD}_{45 \mathrm{RB}^{\text {low }} 52,55}, \mathrm{CD} 127^{56,57}$, and the glucocorticoid-induced tumour necrosis factor receptor family-related gene $(\mathrm{GITR})^{58}$, has been used for the identification of certain types of nTregs, to date, the best marker for distinguishing nTregs from effector T cells is Foxp3. This is a member of the forkhead family of transcription factors ${ }^{59}$, and the Foxp3 gene expression is critical for the cells' suppressive function ${ }^{55}$.

\subsubsection{Mechanisms of Foxp ${ }^{+}$nTreg mediated suppression}

Foxp $^{+}$nTregs potently suppress the activation, proliferation and effector functions of various cells, including conventional $\mathrm{CD}^{+}$and $\mathrm{CD}^{+} \mathrm{T}$ cells, DCs and $\mathrm{B}$ cells ${ }^{59}$. It is possible that the Foxp $3^{+}$nTregs control a variety of cell responses by implementing multiple modes of suppression. The putative mechanisms of nTreg-mediated suppression include the inhibition of IL-2 transcription ${ }^{59}$, IL-10 production, surface CTLA-4 expression ${ }^{60}$, co-stimulatory molecule blockade ${ }^{60}$, surface TGF- $\beta$ expression, granzyme $\mathrm{B}$ and perforin expression ${ }^{61}$, and inhibitory cytokine IL-35 production ${ }^{62}$. Furthermore, it is believed that the primary mechanism of nTreg suppression is cell-contact dependent. Despite great efforts, the molecules involved in the cell-contact mediated suppression are yet to be identified ${ }^{59}$.

\subsubsection{Interaction between Tregs and APC}

Although the primary mechanism utilised by Tregs to suppress $\mathrm{T}$ cell responses is believed to be contact dependent ${ }^{44,63}$, it is not clear with what cell types Tregs interact. There are two possible scenarios by which Tregs carry out their suppressive function. In the first scenario, Tregs interact with effector $\mathrm{T}$ cells and suppress cell activation or induce anergy/cell death directly. In the second scenario, Tregs interact with APCs and change the expression of certain surface molecules on the APCs. Such changes are necessary for the tolerogenic activity of the APCs, and these tolerogenic APCs in turn induce anergy in responder $\mathrm{T}$ cells.

Although Tregs may act directly on effector $\mathrm{T}$ cells, the following evidence from several studies support the idea that Tregs primarily carry out their suppressive function via the interaction with APCs. First, in vitro suppressive assays have shown that when the ratio of Tregs to effector $\mathrm{T}$ cells is $1: 1$, the proliferation of effector $\mathrm{T}$ cells is greatly suppressed $^{60,64}$. However, the ratio between Tregs and effector $\mathrm{T}$ cells rarely reaches 1:1 
in vivo. Therefore, it is more likely that Tregs carry out their suppressive function by interacting with APCs. Second, two-photon laser-scanning microscopy data have provided direct evidence that Tregs contacted APCs and not effector T cells in vivo ${ }^{65}$. In this study, Tregs were found to directly interact with the DCs bearing a specific antigen. In contrast, no detectable stable associations between Tregs and effector $\mathrm{T}$ cells were observed. Third, a recent in vitro study showed that Tregs contact APCs and have a direct effect on these APCs. In the presence of TCR stimulation, Tregs preferentially form aggregates on APCs rather than effector $T$ cells ${ }^{66}$. Such engagement down-regulates the expression of CD80 and CD86 on the APCs. Treg/APC aggregates are dependent on LFA-1 and the down-regulation of CD80/CD86 is dependent on CTLA-4 ${ }^{60,66}$. Moreover, Tregs have been shown to induce macrophages to express B7-H4 molecules ${ }^{67}$, indicating that Tregs are able to change cell surface molecule expression on different subsets of APCs. Despite these leading studies, the precise cell-cell interactions between Tregs and APCs remain unidentified. This uncertainty may be due, in part, to the difficulty of defining the precise interactions between diverse APC and Treg subsets.

It has been suggested that different APCs, in particular DC subsets, have divergent abilities for the induction of tolerance through Tregs. For example, matured plasmacytoid DCs (pDCs) ${ }^{68}$ and immature myeloid DCs (mDCs) ${ }^{69}$ have the ability to induce Treg cells, whereas mature mDCs can induce the proliferation of Tregs in vitro $^{70}$. Therefore, certain types of APCs may preferentially promote Treg differentiation and enhance Treg-mediated suppression. A better understanding of the rules that govern Treg-APC relationship will enable us to better manage the immune response in autoimmunity and other disease settings.

\subsection{Myeloid derived suppressor cells}

In recent years, a population of $\mathrm{CD} 11 \mathrm{~b}^{+} \mathrm{Gr}-1^{+}$suppressive myeloid cells have been described in different diseases ${ }^{71-74}$ and named "myeloid-derived suppressor cells (MDSCs) ${ }^{45,75,76}$. These cells have been shown to inhibit both innate and adaptive immunity and are considered a major contributor in assisting tumours to escape immune recognition ${ }^{45,75,76}$. MDSCs are a heterogeneous immature myeloid cell population comprised of DC, macrophage, and /or granulocyte precusors ${ }^{45,76}$. These cells have 
been identified in most tumour environments and undergo dramatic expansion during tumour development ${ }^{77}$.

The MDSCs derived from tumours-bearing mice suppress $T$ cell responses by multiple mechanisms. In the in vitro setting, MDSCs are able to suppress $\mathrm{T}$ cell responses via up-regulation of $\mathrm{NO}$ production $^{78}$. NO regulates $\mathrm{T}$ cell activation via reversible disruption of the Janus kinase (Jak)/signal transducer and activator of transcription (STAT) 5 signalling pathway ${ }^{79}$. MDSCs can also suppress T cells by uptake of arginine, a conditionally essential amino acid that is critical for $\mathrm{T}$ cell function ${ }^{80-83}$. Other suppressive mechanisms have also been reported. For example, it has been suggested that the suppressive activity of MDSC's is mediated by Tregs ${ }^{74}$. These suppressive mechanisms have been shown to act in divergent ways in different disease models. Even in the same tumour model, the MDSCs have been reported to suppress immune responses using different mechanisms ${ }^{71}$. The reason for the contrasting results may be due to the heterogeneity of the suppressive cell subsets within the Gr- $1^{+} \mathrm{CD} 11 \mathrm{~b}^{+}$cell population. Identification of the specific suppressor cell subpopulation in different pathological conditions would help provide better-targeted therapeutic interventions.

Although tumour-induced MDSCs are potent suppressors of the immune system, the question whether MDSCs exist in healthy individuals remains unanswered. Results from measuring the in vitro suppressive activity of the $\mathrm{Gr}-1^{+} \mathrm{CD} 11 \mathrm{~b}^{+}$cells from tumour-free mice are not consistent. Most reports indicate that the $\mathrm{Gr}-1^{+} \mathrm{CD} 11 \mathrm{~b}^{+}$cells from tumour-free mice are not suppressive $\mathrm{e}^{71,74,84-86}$. However, one paper demonstrates that the splenic $\mathrm{Gr}-1^{+} \mathrm{CD} 11 \mathrm{~b}^{+}$cells from tumour-free mice are able to suppress $\mathrm{CD}^{+} \mathrm{T}$ cell response ${ }^{87}$. Additional studies are clearly needed to clarify whether MDSC exist in disease-free individuals and play a role in homeostasis and maintenance of tolerance to self-antigens.

\subsection{Autoimmune disease}

As discussed above, the body utilises a variety of mechanisms to protect its cells against its own immune responses. However, if one or more of these tolerance mechanisms fail, autoimmunity can result. The diseases that result from an autoimmune response are termed as an autoimmune disease. Autoimmune diseases include more than 70 different disorders and are widely variable in terms of targeted tissues, age of onset, and nature of 
the autoimmune responses. Prominent examples include MS, type 1 diabetes, systemic lupus erythematosus (SLE) and rheumatoid arthritis (RA) ${ }^{35,88-90}$.

The risk factors for the development of autoimmune diseases are still largely unknown ${ }^{90}$. It is believed that autoimmune diseases result from multiple factors, which either determine the disease susceptibility (e.g. genetic factors such as certain human MHCII molecules ${ }^{91,92}$ ) or trigger autoimmune responses (e.g. environmental factors ${ }^{88,90}$ ). Among the environmental factors that trigger autoimmunity, infections have drawn most attention and a variety of hypotheses have been put forward to explain the role of infections in the onset of autoimmunity. The possible mechanisms by which infectious agents may provoke autoimmunity are diverse. These mechanisms include antigenic cross-reactivity between the micro-organisms and the host tissues (i.e. molecular mimicry) ${ }^{93}$; the infection of microbial superantigens that stimulate $\mathrm{T}$ lymphocytes (some of which could be autoreactive and participate in the development of autoimmune diseases) ${ }^{94}$; direct infection of immune cells (for example, infecting microglial cells with Theiler's murine encephalomyelitis virus can up-regulate the cells' expression of MHC and co-stimulatory molecules and result in the enhancement of these cells' ability to function as APCs) $)^{95}$; and deviation of the balance between Th subsets $^{96}$. Other environmental factors implicated include trauma, smoking and nutritional factors, all of which tend to impair the immune system ${ }^{35,97,98}$.

\subsection{Multiple sclerosis}

MS is a chronic inflammatory autoimmune disease, in which the immune response is directed against the myelin sheath that surrounds the nerve axons in the CNS. The disease usually starts between 20 to 40 years of age for humans and leads to substantial disability through deficits of motor sensory and cognitive nerve function. The socioeconomic importance of MS is second only to trauma in young adults ${ }^{88,98}$. In New Zealand, more than 4,000 patients have MS and approximately 200 people are diagnosed with the disease every year ${ }^{99}$.

MS is characterised as degenerative and inflammatory changes within the brain and spinal cord, which are associated with the formation of sclerotic plaques due, at least in part, to abnormal hardening and fibrosis of the neuronal myelin sheath ${ }^{88}$. The symptoms of MS are diverse and include fatigue, depression, cognitive dysfunction, tremor, 
disturbance in speech and vision, and loss of bowel or bladder control ${ }^{88}$. Although the clinical course is highly variable, within 15 years of onset of symptoms, more than $80 \%$ of MS patients are moderately disabled to totally paralysed ${ }^{99,100}$.

There are two major forms of MS, relapsing-remitting form (RRMS) and primary progressive form of MS (PPMS). Approximately 85-90\% of MS patients develop RRMS. People with RRMS have unpredictable relapses, but during the remission between the relapses, return to normal. After around 15 years, most of the RRMS patents will progress to secondary progressive MS (SPMS). The remaining 10-15\% of the patients have PPMS that is characterised by a steady progression from its onset without relapse or remission ${ }^{98}$. It is unclear which factors are responsible for the different disease courses.

\subsubsection{Etiology of MS}

It is believed that MS develops in genetically susceptible individuals but requires additional environmental triggers ${ }^{98}$. Strong evidence for potential genetic contributions comes from familial studies as MS risk increases with the degree of shared genetic background $^{101}$. Several studies indicate that many different genes contribute to the risk of developing MS. However, all of these genes are MHCII alleles and the specific genes that confer risks are HLA-DR ${ }^{102}$ and HLA-DQ ${ }^{91}$. Haplotypes DQA1*0102, DQB1*0602, DRB1*1501 and DRB5*0101 have been mapped with high risks ${ }^{103}$. However, non-twin siblings of affected individuals have a concordance of only $1-2 \%$ for developing MS, dizygotic twins 2-3\% and monozygotic twins 20-35\% ${ }^{101}$. This relatively low concordance rate indicates an important role of non-genetic factors in MS etiology.

The geographical distribution of MS disease prevalence increases with latitude, and the highest is at $40^{\circ}$ in both hemispheres ${ }^{100}$. Prevalence and migration studies provide evidence for the involvement of pollution, diet, smoking habits, UV light exposure and virus infections in MS development ${ }^{88,98,100,104}$. Among the putative environmental factors, both lifestyle and infectious agents have been proposed to contribute to MS. Moreover, hormonal variables are suggested as potential risk factors since twice as many women are affected by $\mathrm{MS}^{98}$ and a large body of evidence indicates the therapeutic potential of estrogens in treating $\mathrm{MS}^{105}$. 


\subsubsection{Immunology of MS}

MS is a typical organ-specific autoimmune disease ${ }^{98,100}$ but questions remain about the nature of the immune attack on the CNS. It is believed that the invasion of autoreactive myelin-specific $\mathrm{T}$ lymphocytes into the CNS plays a central role both in the disease initiation and progression ${ }^{88,98,106}$. Additionally, the strongest known genetic association with $\mathrm{MS}$ is the HLA-DR2 molecule $(\mathrm{DRB} 1 * 1501)^{103,107}$, which implicates the importance of the MHCII restricted CD4+ T cells in MS pathogenesis. In some MS patients, myelin basic protein peptides (MBP) are presented by the HLA-DR2 molecules at sites of demyelination ${ }^{108}$ and $\mathrm{T}$ cells with TCRs specific for MBP have been found in MS brain lesions ${ }^{109}$.

Extrapolating from a large body of research, it appears that the immune attack in MS can be divided into five phases (induction, migration, reactivation, tissue injury and resolution $)^{88,97,98,100,110-114}$. The first phase involves the activation of myelin-specific $\mathrm{T}$ cells to become pro-inflammatory effector $\mathrm{T}$ cells outside the CNS. The exact mechanism by which these autoreactive $\mathrm{T}$ cells become sensitised to myelin remains unclear. One hypothesis involves molecular mimicry and suggests that autoreactive $\mathrm{T}$-cell clones are activated by foreign antigens, such as viruses resembling the antigenic structure of myelin proteins ${ }^{93,115}$.

The second and third phases involve the transmigration of autoreactive $\mathrm{T}$ lymphocytes across the blood-brain barrier (BBB) (the second phase) and the reactivation of these autoreactive $\mathrm{T}$ cells upon encountering myelin proteins in the CNS (the third phase). Although it is believed that a small number of immune cells might periodically cross the $\mathrm{BBB}^{100}$, brain parenchyma does not typically include a large number of immune cells. Upon encountering myelin proteins, the autoreactive $\mathrm{T}$ cells start producing inflammatory cytokines that induce local effects and activate the endothelial cells in the postcapillary venules so that a second wave of immune cells can be recruited from the blood to the CNS.

The fourth phase of immune attack is characterised by tissue injury in the CNS manifesting as macrophage infiltration, myelin disruption and axonal damage. Although myelin and/or oligodendrocytes themselves disappear from the MS plaque, the exact mechanism of the myelin damage remains unknown. It is likely that oxygen radicals, 
complement cascade, $\mathrm{CD}^{+}$cells, $\mathrm{CD} 8^{+}$cells, macrophages, antibodies and activation of local glial cells all play a role in this injury process ${ }^{98}$. In the fifth phase, the inflammatory process gradually resolves, ultimately leaving behind scars characterised by the absence of myelin, partial losses of axons in some cases and dense astrocytic scars.

\subsubsection{Tregs and MS}

In healthy individuals, Tregs suppress the activation and expansion of self-reactive $\mathrm{T}$ cells, thereby preventing autoimmune diseases ${ }^{44}$. However, Tregs from the peripheral blood of MS patients are found to have a significant deficiency in their suppressive function compared with healthy donors ${ }^{116}$. It is proposed that this functional deficiency of Tregs leads to uncontrolled self-reactive $\mathrm{T}$ cell responses in vivo, which result in $\mathrm{MS}^{116,117}$. Therefore, a method that restores the inhibitory function of Tregs in patients with MS has the potential to be used clinically to prevent and treat MS.

\subsubsection{Current medication of MS}

There is no cure for MS. Currently there are six disease-modifying treatments available for MS in different countries. Three of them are IFNs, two formulations of IFN beta-1a (Avonex and Rebif) and one of IFN beta-1b (Betaferon). The remaining three are glatiramer acetate (Copaxone), mitoxantrone and natalizumab (Tysabri) ${ }^{118}$.

In New Zealand, only Betaferon, Avonex and Copaxone are fully subsidised by the government $^{119}$. Betaferon and Avonex help prevent relapses but are only able to reduce disease exacerbations in $30 \%$ of cases and have a less certain effect on progressive disability. The side effects such as flu-like symptoms and the frequency of subcutaneous injection deter some patients from the treatment ${ }^{41,100,120}$. Copaxone has been shown to have similar effects to IFN-beta in reducing MS attacks, but has a more favourable side effect profile. Copaxone is a random copolymer of the four amino acids Ala, Lys, Glu and Thy with various lengths at fixed molar ratios at 4.5: 3.6: 1.5:1, respectively ${ }^{98}$. The initial purpose of synthesising Copaxone was to mimic myelin basic protein (MBP), a major encephalitogen in MS pathogenesis, for the induction of EAE. However, Copaxone was found to suppress EAE instead ${ }^{121}$ and was developed as a treatment for MS. The mechanism by which Copaxone inhibits MS remains unresolved. Mechanisms such as Th2 response induction ${ }^{122-124}$, competition with self-antigen for the 
MHCII binding ${ }^{125,126}$, Treg induction ${ }^{127,128}$, demyelination arrest and remyelination ${ }^{129}$ have all been suggested. All the above three medical treatments are expensive, must be given by regular injections and require life-long use to remain effective. Moreover, clinical studies have revealed progressive disability in patients with these three treatments ${ }^{41,130}$.

\subsection{EAE mouse model}

Due to limited availability of human samples during active stages of MS, much of the present knowledge about the disease is from studies in animal models. EAE is the best defined murine experimental model for MS that resembles the immunopathology of human $\mathrm{MS}^{131}$. EAE has been utilised extensively to study the cause and investigate the potential treatments of $\mathrm{MS}^{132}$. The majority of the current therapies being planned for phase II and III trials of MS were first examined in $\mathrm{EAE}^{113,133}$.

EAE is routinely induced in laboratories by injecting naïve susceptible mice with defined protein components of the myelin sheath together with adjuvant. MBP, myelin oligodendrocyte glycoprotein (MOG), proteolipoprotein (PLP), myelin associated glycoprotein (MAG) and S-100 protein are the major known CNS antigens and elicit EAE in mice ${ }^{98}$. In this thesis, EAE was induced by injecting the synthetic peptide comprising amino acids 35-55 of MOG $\left(\mathrm{MOG}_{35-55}\right)$ emulsified in complete Freund's adjuvant (CFA) to $\mathrm{C} 57 \mathrm{BL} / 6 \mathrm{~J}$ mice bearing $\mathrm{H}-2^{\mathrm{b}}$. This is a chronic progressive form of $\mathrm{EAE}$ and the disease is non-remitting ${ }^{134}$.

\subsubsection{Pathogenic $\mathrm{CD}^{+} \mathrm{T}$ cells in EAE}

EAE is a $\mathrm{CD}^{+} \mathrm{T}$ cell-mediated autoimmune disease of the $\mathrm{CNS}^{112}$. This conclusion is based on the observation that EAE could be transferred by in vitro reactivated myelin-specific $\mathrm{CD}^{+} \mathrm{T}$ cells, but could not be transferred by either antibodies or other cells ${ }^{135}$. In the past, a widely accepted theory was that the activation of autoreactive $\mathrm{CD}^{+} \mathrm{T}$ cells specific for myelin antigens and the differentiation to Th1 lineage were crucial for the development of EAE. However, this theory has been reconsidered upon the recent discovery of Th17 cells and their involvement in $\mathrm{EAE}^{15,136,137}$.

Originally $\mathrm{CD}^{+}$Th cells were divided into Th1 and Th2 cells. Th1 cells are most important for clearance of intracellular pathogens whereas Th2 cells play a major role in 
defending the body against helminths and some extracellular pathogens. As shown in Figure 1.1, Th1 cells produce pro-inflammatory cytokines, such as IFN- $\gamma$. These cytokines augment the cytotoxicity of macrophages and proliferation of CTLs. Differentiation of Th1 cells is driven by IL-12, a cytokine produced by APCs such as macrophages and DCs. Th2 cells produce numerous cytokines such as IL-4, IL-5 and IL-13. These Th2 cytokines are crucial for humoral immunity by stimulating B cell proliferation, antibody class switching, and increasing antibody production. Many of the Th2 cytokines also act as part of regulatory mechanisms by counterbalancing Th1 responses $^{13,138,139}$.

Originally Th1 cells were believed to mediate EAE, but EAE was exacerbated in IFN- $\gamma$ deficient and IFN- $\gamma$ receptor deficient mice ${ }^{140,141}$. This raised the question that another subset of $\mathrm{T}$ cells different from Th1 cells might be required for the development of EAE. Recently, an additional $\mathrm{CD}^{+}{ }^{+}$Th cell subset was discovered and defined by the production of IL-17-Th17 cells ${ }^{15}$. The master transcription factor for Th17 cells is ROR $\gamma \mathrm{t}^{142}$ and the primary function of Th17 cells appears to be the clearance of pathogens that cannot be effectively dealt with by Th1 or Th2 cells ${ }^{15,143}$. Th17 cells and their cytokines have been recognised to play a crucial role in EAE disease development ${ }^{137}$, because the absence of IL-17 in IL-17 deficient mice ${ }^{144}$ and in mice treated with IL-17 neutralising antibody ${ }^{145}$ results in considerable amelioration of EAE symptoms. Additionally, in adoptive transfer experiments, IL-17 producing $\mathrm{CD}^{+} \mathrm{T}$ cells induce EAE whereas the ones that only produced IFN- $\gamma$ cannot ${ }^{146}$, indicating that Th17 cells are the pathogenic cells of EAE. However, recent evidence also indicates that Th1 cells also play a pathogenic role in EAE, in that Th1 cells preferentially infiltrate into the non-inflamed CNS to initiate inflammation and facilitate the recruitment of Th17 cells $^{147}$. Therefore, both Th1 and Th17 cell populations are involved in EAE development.

As shown in Figure 1.1, a mixture of cytokines that includes IL-6, TGF- $\beta$ and IL-23 have been found to be important for Th17 cell development and differentiation ${ }^{148,149}$. IL-6, produced mainly by macrophages and DCs, is an obligatory differentiation factor for Th17 cells and is important for EAE development ${ }^{150,151}$. IL-6 deficient mice do not generate Th17 cells and are resistant to $\mathrm{EAE}^{152,153}$. In addition, TGF- $\beta$ is also necessary 
for in vivo Th17 differentiation. Mice defective in TGF- $\beta$ signalling do not develop Th17 cells or succumb to $\mathrm{EAE}^{150}$. Moreover, TGF- $\beta$ induces the differentiation of Tregs in the absence of IL- $6^{146}$, while the combination of IL- 6 with TGF- $\beta$ favours Th17 development and inhibits Foxp3 expression ${ }^{152,154}$. Therefore, the interplay between Tregs and Th17 cells extends beyond their functions, as their development is mutually antagonistic.

IL-23, produced by DCs and other APCs, is required for full and sustained differentiation of Th17 cells ${ }^{155}$, although the specific mechanism of IL-23 signalling in $\mathrm{T}$ cells remains elusive. Recent studies show that the enhanced up-regulation of ROR $\gamma \mathrm{t}$ in activated $\mathrm{T}$ cells is dependent on STAT3 induction by IL-23 ${ }^{156,157}$ and IL-23 signalling is required for Th17 cells pathogenic capacity ${ }^{158}$. Selective deficiency of IL-23 in mice resulted in fewer Th17 cells and these mice developed attenuated AEE $^{155}$.

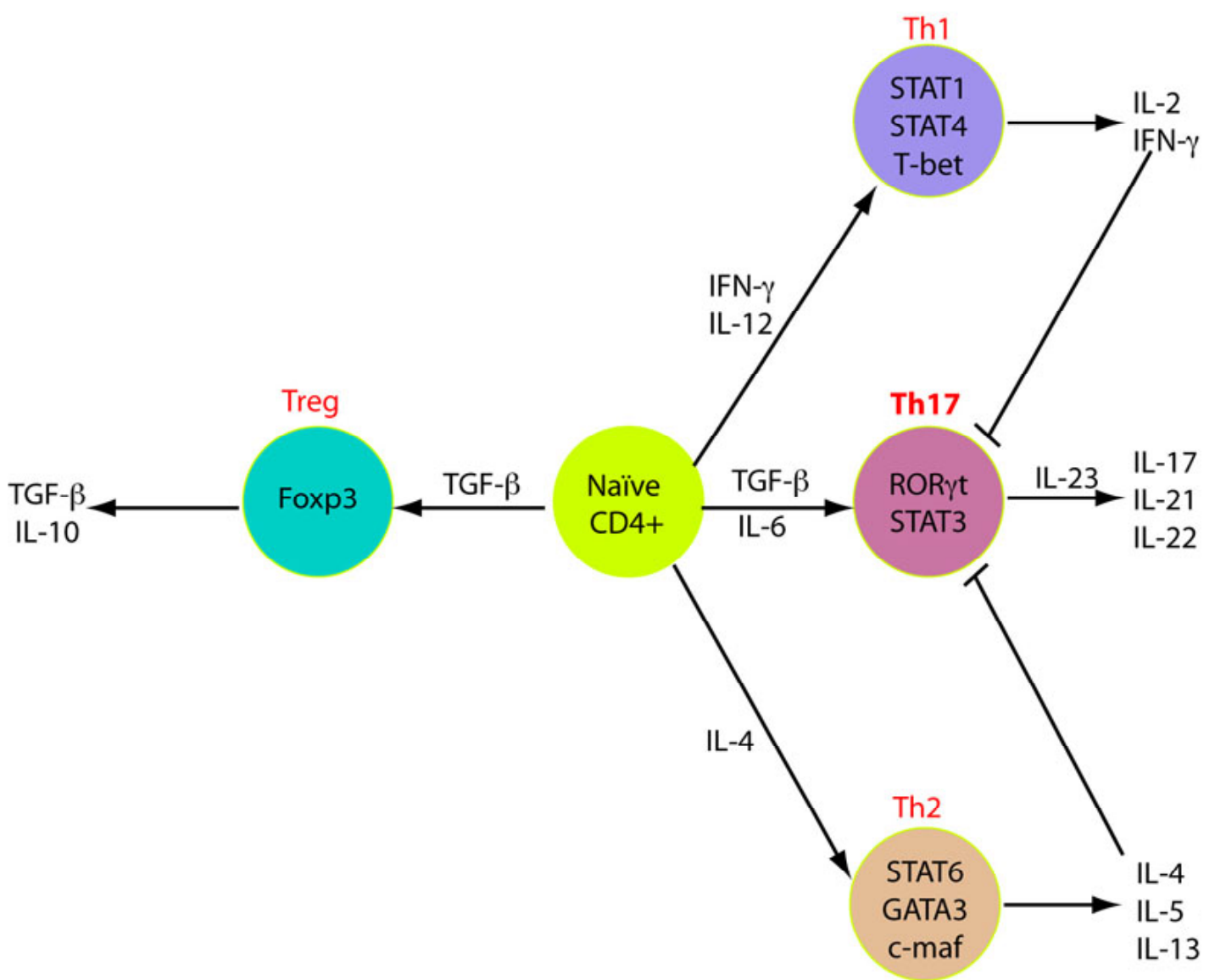

Figure 1.1 Differentiation of $\mathrm{CD}^{+} \mathrm{T}$ cell lineages

Naïve $\mathrm{CD}^{+} \mathrm{T}$ cells differentiate into Tregs and three subsets of effector T cells-Th1, Th2 and Th17. The differentiation of the $\mathrm{CD} 4^{+} \mathrm{T}$ cell subsets is dependent on the cytokine milieu (This graph is adapted and adjusted from Bettelli et al $2007^{159}$ ). 


\subsubsection{Tregs in EAE}

Tregs are believed to play a key role in the disease regulation in EAE. Adoptive transfer of polyclonal naïve $\mathrm{CD} 25^{+} \mathrm{T}$ cells limits the severity of $\mathrm{EAE}^{160,161}$ and injecting mice with anti-CD25 monoclonal antibody accelerated EAE development ${ }^{162}$. Taken together, these experiments clearly illustrate that Tregs profoundly suppress EAE development.

It is believed that autoantigen-specific Tregs are superior over polyclonal Tregs for treating autoimmune diseases. This belief is based on the observation that the Tregs specific for a pancreatic islet antigen are more potent compared to polyclonal Tregs in controlling diabetes in NOD mice ${ }^{163,164}$. There are two approaches by which autoantigen-specific Tregs could be used therapeutically. The first would be by expanding the autoantigen-specific Treg numbers in vitro and reintroducing them into mice, with the aim of increasing the frequency of Tregs for suppressing the disease. Adoptive transfer of in vitro expanded islet-reactive Tregs has been shown to greatly inhibit type 1 diabetes in NOD mice ${ }^{164}$, indicating the potential for in vitro expanded myelin-specific Tregs to suppress EAE. The second approach would be the administration of an appropriate therapeutic agent that enhances the suppressive effects of autoantigen-specific Tregs, by increasing their frequency or suppressive function in vivo. Although several recent reports have demonstrated that activating Tregs in vivo using a variety of drugs promote the protection of mice against $\mathrm{EAE}^{165-167}$, to my knowledge, the evidence for antigen-specific Treg in vivo expansion is limited. Nevertheless, a study carried out in our laboratory demonstrated that treating mice with a compound consisting of a modified superantigen (mSAg) and $\mathrm{MOG}_{35-55}$ could suppress $\mathrm{EAE}$ in a $\mathrm{MOG}_{35-55}$ specific manner and the mechanism is hypothesised to be expansion or activation of $\mathrm{MOG}_{35-55}$ specific Tregs in vivo ${ }^{168}$. These studies highlight the potential of Treg-based therapies with antigen-specific properties for treating MS and other autoimmune diseases.

\subsubsection{Suppressive myeloid cells in EAE}

Despite a number of reports on the suppressive myeloid cells such as MDSCs in tumours, these cells have been poorly studied in other diseases. To my knowledge, there has only been one report describing a suppressive myeloid cell population in autoimmune diseases. Zhu et al ${ }^{169}$ have described a splenic $\mathrm{CD} 11 \mathrm{~b}^{+} \mathrm{Ly} 6 \mathrm{C}^{+}$myeloid suppressor cell population in the EAE model. These splenic CD $11 \mathrm{~b}^{+} \mathrm{Ly}_{6 \mathrm{C}^{+}}$cells from 
Balb/c mice induced with EAE profoundly suppress the proliferation and cytokine production of $\mathrm{CD}^{+}$cells pre-activated with anti-CD3/anti-CD28. The suppression of $\mathrm{CD}^{+} \mathrm{T}$ cell response is due to apoptosis in the proliferating $\mathrm{CD}^{+} \mathrm{T}$ cells. Both inducible nitric oxide synthase (iNOS) expression in the splenic CD11 b ${ }^{+} \mathrm{Ly}_{6} \mathrm{C}^{+}$cells and IFN- $\gamma$ production of $\mathrm{T}$ cells are essential for this $\mathrm{CD} 11 \mathrm{~b}^{+} \mathrm{Ly}_{6 \mathrm{C}}{ }^{+}$cell-mediated $\mathrm{T}$ cell suppression $^{169}$. This study highlights the potential of myeloid suppressor cell-based therapy for treating autoimmune diseases.

It remains unclear if the splenic $\mathrm{CD}_{11} \mathrm{~b}^{+} \mathrm{Ly} 6 \mathrm{C}^{+}$cells from naïve mice have a

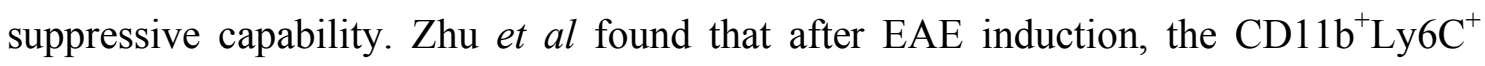
cells accumulate in the bone marrow, blood, spleen, and CNS. This increase is not $\mathrm{MOG}_{35-55}$ specific, because immunisation with CFA/pertussis toxin (PTxn) alone could induce a similar expansion of this cell population ${ }^{169}$. This finding is contradictory, because the accumulation of the suppressive $\mathrm{CD} 11 \mathrm{~b}^{+} \mathrm{Ly} 6 \mathrm{C}^{+}$cells corresponds with the progression of EAE. Further studies are required to understand what role these cells play in the pathogenesis of EAE and their therapeutic value for actively inhibiting EAE.

EAE is a $\mathrm{CD}^{+} \mathrm{T}$ cell mediated disease ${ }^{112}$ and the myeloid suppressor cells have been found to suppress $\mathrm{CD}^{+} \mathrm{T}$ cell proliferation and cytokine production ${ }^{169}$, therefore, a therapy targeting myeloid suppressor cell populations might help to induce or restore immunosuppression during EAE. While the existence of myeloid suppressor cells in mice and humans is accepted ${ }^{170}$, much remains to be learnt about the suppressive mechanisms of these cells and whether their potential can be exploited as an immunotherapy for treating human MS and other autoimmune diseases.

\subsection{Superantigen}

Bacterial superantigens are a family of structurally related protein toxins produced exclusively by three pathogenic species, Staphylococcus aureus, Streptococcus pyogenes and Streptococcus equi. In contrast to the traditional peptide antigens, superantigens bind to MHCII molecules outside the peptide binding grooves and to all TCRs bearing a particular $\mathrm{V} \beta$ chain ${ }^{171}$. Moreover, superantigens require no or little intracellular processing within the APCs in order to associate with MHCII on the cell surface $^{172}$. The simultaneous binding of superantigen to MHCII and TCR stimulates a large number of $\mathrm{T}$ cells. Conventional antigens presented by MHCII activate 1 in 
$10^{5}-10^{6} \mathrm{~T}$ cells whereas superantigens stimulate between $2-20 \%$ of all $\mathrm{T}$ cells ${ }^{173}$. The over-activation of $\mathrm{T}$ cells leads to high systemic level of cytokine production such as TNF- $\alpha$ and IL-1 $\beta$ and other T cell mediators such like IL-2 and IFN- $\gamma$. The over-production of these cytokines results in indiscriminate inflammation characteristic of fever and toxic shock ${ }^{171-175}$. The most potent superantigen discovered so $\operatorname{far}^{171}$ is Streptococcal mitogenic exotoxin $Z$ variant 2 (SMEZ-2), which is secreted by the Gram positive bacterium Streptococcus pyogenes $\mathrm{T} 1^{174}$ and has been implicated in Streptococcus-mediated disease ${ }^{171}$.

As superantigens are able to activate a large number of $\mathrm{T}$ cells, superantigens are suggested to play a role in activating auto-reactive $\mathrm{T}$ cells and initiating autoimmune diseases ${ }^{176}$. It has been demonstrated that some superantigens, e.g. Staphylococcal enterotoxin A (SEA) and Staphylococcal enterotoxin B (SEB) are able to exacerbate $\mathrm{EAE}^{177}$. Paradoxically, it is also reported that the administration of superantigens can suppress EAE. For example, pre-treatment with SEB blocks MBP induced EAE ${ }^{178}$, and the administration of high doses of Staphylococcal enterotoxin E (SEE) also suppresses EAE. The mechanism for the superantigen-mediated EAE suppression is proposed to be the deletion or anergy of auto-reactive $\mathrm{T}$ cells caused by the prolonged exposure to superantigens ${ }^{179-181}$. A study ${ }^{168}$ carried out in our laboratory showed that the administration of a modified version of SMEZ-2 superantigen conjugate with the $\mathrm{MOG}_{35-55}$ peptide $\left(\mathrm{SM}_{-} \mathrm{MOG}_{35-55}\right)$ into mice suppressed $\mathrm{EAE}$ in a $\mathrm{MOG}_{35-55}$ specific manner. Hence, the modified superantigen ( $\mathrm{mSAg}$ ) provides a potential tool for developing an immunotherapy for EAE and human MS.

\subsection{Aims and hypotheses}

The overall goal of this thesis is to explore the potential of immunosuppressive cells to prevent/suppress EAE, the murine model for MS, with the ultimate goal of developing a potential immunotherapy for MS. There are three specific aims in this thesis:

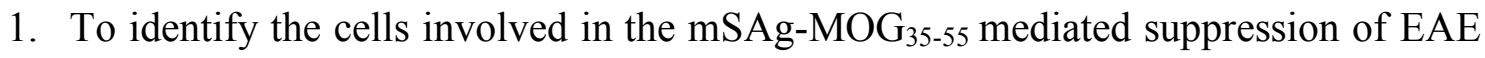
and to understand the cellular and molecular changes during the mSAg-MOG $35-55$ mediated immune suppression in vivo.

2. To investigate the potential for targeting of suppressor cells by $\mathrm{mSAg}-\mathrm{MOG}_{35-55}$ for enhanced EAE suppression 
3. To evaluate the therapeutic potential of $\mathrm{mSAg}-\mathrm{MOG}_{35-55}$ in EAE.

In considering these three aims, I put forward the following alternative hypotheses:

Hypothesis 1: $\mathrm{SM}-\mathrm{MOG}_{35-55}$ expands and/or activates a population of $\mathrm{MOG}_{35-55}$-specific Tregs in vivo, which inhibit the self-reactive disease-causing $\mathrm{CD} 4^{+} \mathrm{T}$ cells.

Hypothesis 2: SM-MOG $35-55$ activates non-Treg suppressor cells in vivo, which inhibit the self-reactive disease-causing $\mathrm{CD}^{+} \mathrm{T}$ cells. 


\section{Chapter 2:}

\section{Materials and Methods}




\subsection{Mice}

\subsubsection{Maintenance and ethical approvals}

All mice were bred and maintained on standard laboratory food and water ad libitum in the Biomedical Research Unit of the Malaghan Institute of Medical Research. All animal studies were approved by the Victoria University of Wellington Animal Ethics Committee and performed in accordance with the guidelines of the Victoria University of Wellington Animal Ethics Committee, New Zealand (Ethics approval numbers 2007-R8M and 2006-R19).

\subsubsection{Mouse strains}

C57BL/6J mice bearing H-2 ${ }^{\mathrm{b}}$ were originally purchased from the Jackson Laboratory (Bar Harbour, ME, USA) and were maintained by inbreeding.

Foxp3-GFP mice harbouring a GFP-Foxp3 fusion protein reporter knock-in allele (B6×129, Foxp3-GFP-g2) were imported from the University of Washington (Seattle, WA, USA), and have been described by Fontenot et al ${ }^{182}$. The Foxp3-GFP transgenic mice utilised in this study had been backcrossed to C57BL/6J for more than ten generations and were maintained by breeding C57BL/6J males with Foxp3-GFP transgenic females.

2D2 mice expressing transgenic TCRs specific for the $\mathrm{MOG}_{35-55}$ peptide (MEVGWYRSPFSRVVHLYRNGK) presented by IA $^{\mathrm{b}}$ were obtained from Harvard Medical School (Boston, MA, USA). The 2D2 mice were derived by microinjection of cDNA encoding a TCR with reactivity to $\mathrm{MOG}_{35-55}$ peptide expressing the V $\alpha 3.2$ and V $\beta 11$ TCR chains into oocytes of C57BL/6J mice ${ }^{183}$. The transgenic mice were maintained by breeding C57BL/6J males with 2D2 TCR transgenic females.

OTII mice expressing the V $\alpha 2$ and $V \beta 5.2$ chain TCRs specific for $\mathrm{OVA}_{323-339}$ peptide (ISQAVHAAHAEINEAGR) presented by IA $^{\mathrm{b}}$ were originally obtained from Professor Frank Carbone, Melbourne University (Melbourne, Victoria, Australia). The OTII mice were generated using a combination of cDNA- and genomic DNA- based construct $^{184}$. The OTII transgenic mice utilised in this study have been backcrossed to C57BL/6J for more than eight generations and were maintained by inbreeding. 
B6.SJL-PtprcaPep3b/BoyJARC mice were created by backcrossing the inbred strain SJL expressing the Ptprca gene (CD45.1) onto the C57BL/6J background. This strain was obtained from the Animal Resources Centre (Canning Vale, WA, Australia) as CD45 congenic pairs for CD45.2 $2^{+}$C57BL/6J mice, and maintained by inbreeding.

$\mathrm{B} 6 \mathrm{AaO} / \mathrm{AaO} \mathrm{MHC}$ class II deficient (MHCII-/-) mice ${ }^{185}$ were created by targeted mutation of MHC class II gene Aa in embryonic stem cells derived from C57BL/6J mice. This strain was provided by Dr. H. Bluethmann (Hoffmann-La Roche, Switzerland) and was maintained by inbreeding.

Indoleamine-2,3-dioxygenase deficient (IDO-/-) mice are deficient in the enzyme IDO, which is a tryptophan degrading enzyme ${ }^{186}$. IDO-/- mice were generated by replacing exons 3-5 with a targeting vector containing beta-galactosidase and neomycin resistance genes. Exon 2 was also disrupted with a TAG stop codon. The breeding pairs were imported from The University of Sydney (Sydney, NSW, Australia) and were maintained by inbreeding.

The IFN- $\gamma$ deficient (IFN $\gamma-/-)$ strain was generated by targeted disruption of the IFN- $\gamma$ gene. The breeding pairs were imported from the Walter and Eliza Hall Institute of Medical Research (Melbourne, Victoria, Australia) and have been described by Dalton et $a l^{187}$. The IFN $\gamma$-/- mice utilised in this study were maintained by inbreeding.

The Foxp3-GFP $\times 2 \mathrm{D} 2$ strain was generated by crossing female $2 \mathrm{D} 2$ mice with male Foxp3-GFP mice. This strain was maintained by inbreeding.

\subsection{Peptides}

The MOG $_{35-55 \mathrm{C}}$ peptide (H-CMEVGWYRSPFSRVVHLYRNGK-OH) is an $\mathrm{IA}^{\mathrm{b}}$ binding peptide derived from the mouse MOG sequence and corresponds to amino acids 35-55 with an additional cysteine at the N-terminus. This peptide was synthesised by Mimotopes (Victoria, Australia) with a purity of $>97 \%$.

The OVA $_{323-339 \mathrm{C}}$ peptide (H-CISQAVHAAHAEINEAGR-OH) is an $\mathrm{IA}^{\mathrm{b}}$ binding peptide derived from the OVA sequence and corresponds to amino acids 323-339 with 
an additional cysteine at the N-terminus. This peptide was synthesised by Mimotopes (Victoria, Australia) with a purity of $>97 \%$.

\subsection{Conjugation of peptides to $\mathrm{mSAg}$}

The modified SMEZ-2 (mSAgs) were provided by Professor John Fraser (The University of Auckland, New Zealand) at $0.2 \mathrm{mM}$ in $20 \mathrm{mM}$ phosphate buffer $(\mathrm{pH}=6.0)$. These mSAgs are the mutated toxoid form of the wild type SMEZ-2 isolated from S. pyogenes strain $2035^{173}$. SMEZ-2-M1 (SM) has no TCR binding ability and contains three alternations in the TCR binding site: W75L, K182Q, and D42C, whereas SMEZ-2-DM (DM) is defective in both TCR and MHCII binding and contains the mutations Y18A, D42C, H202A and D204A. The D42C mutation introduces an exposed cysteine into the former TCR binding site for direct conjugation of peptides.

\subsubsection{Conjugation of the $\mathrm{MOG}_{35-55 \mathrm{c}}$ peptide to $\mathrm{mSAg}$}

mSAg was incubated with 10 -fold molar excess of $\mathrm{MOG}_{35-55 \mathrm{c}}(2 \mathrm{mM}$ in $0.1 \%$ acetic acid) at room temperature overnight. The $\mathrm{MOG}_{35-55 \mathrm{c}}$ peptide and $\mathrm{mSAg}$ were conjugated through the disulphide bond formed by the cysteine residue. The uncoupled $\mathrm{MOG}_{35-55 \mathrm{c}}$ peptide was removed by spinning through a $10 \mathrm{KDa}$ cut-off vivaspin column 500 (Sartorius, Germany). The conjugation of the mSAg with peptide was subsequently examined by a NuPage gel (Invitrogen, Carlsbad, CA, USA) under non-reducing conditions. Experimental procedures were carried out in accordance with the manufacturer's protocols. Following spinning through a Spin-X polypropylene microcentrifuge tube containing a cellulose acetate membrane filter $(0.22 \mu \mathrm{m}$ pore size $)$ (Corning Inc., Lowell, MA, USA) to remove bacteria and particles, the mSAg-peptide conjugates were stored at $4^{\circ} \mathrm{C}$.

\subsubsection{Conjugation of the $\mathrm{OVA}_{323-339 \mathrm{c}}$ peptide to $\mathrm{mSAg}$}

Different from the $\mathrm{MOG}_{35-55 \mathrm{c}}$ peptide conjugation protocol described above, the $\mathrm{mSAg}$ used for the conjugation with $\mathrm{OVA}_{323-339 \mathrm{c}}$ was first reduced by incubation with $0.5 \mathrm{mM}$ Tris-(2-carboxyethyl)phosphine, hydrochloride (TCEP-HCl) (Thermo scientific, Worcester, MA, USA) at room temperature for 30 minutes. Subsequently, TCEP-HCl was removed by spinning the mSAg-TCEP solution through a $10 \mathrm{KDa}$ cut-off vivaspin column 500 (Sartorius, Germany). The reduced mSAg was then concentrated to $0.2 \mathrm{mM}$ in $20 \mathrm{mM}$ phosphate buffer $(\mathrm{pH}=6.0)$ (Appendix 3) and incubated with 10-fold molar 
excess of $\mathrm{OVA}_{323-339 \mathrm{c}}(2 \mathrm{mM}$ in $0.1 \%$ acetic acid $)$ at room temperature overnight. The

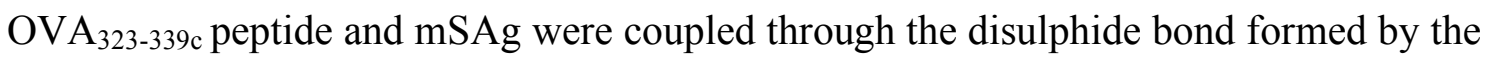
cysteine residues. The uncoupled $\mathrm{OVA}_{323-339 \mathrm{c}}$ was removed by spinning through a $10 \mathrm{KDa}$ cut-off vivaspin column 500 (Sartorius, Germany). The conjugation of the mSAg with $\mathrm{OVA}_{323-339 \mathrm{c}}$ was subsequently examined by a NuPage gel (Invitrogen, USA) under non-reducing conditions. Experimental procedures were carried out in accordance with the manufacturer's protocols. After spinning through a Spin-X polypropylene microcentrifuge tube containing a cellulose acetate membrane filter $(0.22 \mu \mathrm{m}$ pore size $)$ (Corning Inc., USA) to remove bacteria and particles, the mSAg-peptide conjugates were stored at $4^{\circ} \mathrm{C}$.

\subsection{Immunisations for EAE suppression}

\subsubsection{EAE induction and clinical evaluation}

EAE was induced in 8-12 week old C57BL/6J mice by subcutaneous (s.c.) immunisation into hind limb flanks with $0.2 \mathrm{ml}$ of an emulsion containing $50 \mu \mathrm{g}$ $\mathrm{MOG}_{35-55}$ peptide (Mimotopes, Australia) in CFA (Difco Laboratories, Sparks, MD, USA), supplemented with $500 \mu \mathrm{g}$ heat-killed Mycobacterium tuberculosis strain H37RA (Difco Laboratories). The injected mice also received $250 \mathrm{ng}$ pertussis toxin (PTxn) (List Biological, Campbell, CA, USA) in $200 \mu$ PTxn buffer (Appendix 3) on day 1.

Mice were closely monitored every day after immunisation and during the course of EAE development. Paralysed mice were afforded easier access to food and water. Mice were scored according to the following scale: 0 , unaffected; 1, loss of tail tonicity; 2, flaccid tail; 3, flaccid tail and affected hind leg or legs; 4, paralysed hind legs (both); 5, hind body paresis, moribund state. Moribund mice were sacrificed. Mice usually developed clinical signs of acute EAE 12-14 days after immunisation. The incidence of EAE was between $60-100 \%$ in both females and males.

\subsubsection{EAE suppression}

Two versions of $\mathrm{mSAg}-\mathrm{MOG}_{35-55}$ were administered to mice with different routes and doses to suppress EAE. 


\subsubsection{Subcutaneous treatment}

Different doses of mSAg-peptide $\left(\mathrm{MOG}_{35-55}\right.$ or $\left.\mathrm{OVA}_{323-339}\right)$ were mixed with incomplete Freund's adjuvant (IFA) (Difco Laboratories), CFA or PBS and injected s.c. into the necks of $\mathrm{C} 57 \mathrm{BL} / 6 \mathrm{~J}$ mice at different times. In the co-immunisation scheme,

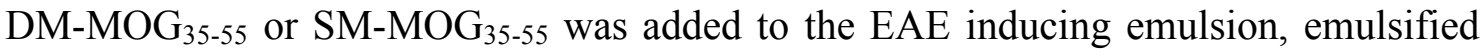
and administered to mice on day zero.

\subsubsection{Epicutaneous treatment}

Mice were anaesthetised by intraperitoneal (i.p.) injection of ketamine/xylazine anaesthetic (Appendix 3) at a volume of $200 \mu \mathrm{l}$ containing $20 \mathrm{mg}$ ketamine (Phoenix Pharm Distributors Ltd, New Zealand) and $0.6 \mathrm{mg}$ xylazine (Phoenix Pharm Distributors Ltd, New Zealand) per mouse. The ear skin on the dorsal side was tape-stripped 12 times to disrupt the epidermal barrier. On the second day, the tape-stripped mice were anaesthetised by i.p injection of $1.6 \%$ pentobarbitone anaesthetic (National Veterinary Supplies Ltd, New Zealand) (Appendix 3) at a dose of $200 \mu 1$ per mouse. An emulsion that consisted of the same volume of PBS and cream

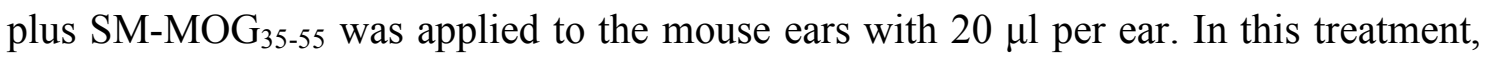
each mouse received $100 \mathrm{ng} \mathrm{SM}-\mathrm{MOG}_{35-55}$.

\subsubsection{Adoptive transfer of cells}

In this study, the purified $\mathrm{CD} 25^{+}$lymphocytes, $\mathrm{F} 4 / 80^{+}$splenocytes or the blood

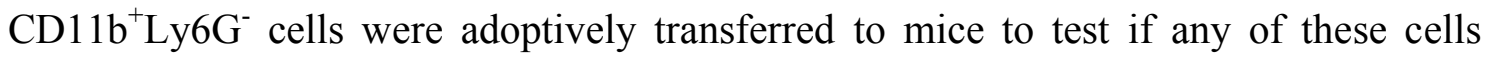
could transfer the suppressive effect of EAE by mSAg-MOG $35-55$. The cells from either

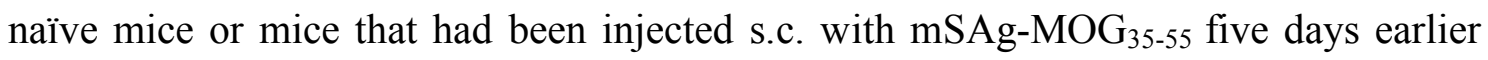
were purified using appropriate cell purification method described in Section 2.7. Varied numbers of the purified cells were subsequently transferred to naïve C57BL/6J or CD45.1 congenic mice by injecting $200 \mu \mathrm{l}$ cells in Hank's buffered salt solution (HBSS) (Invitrogen, USA) into the lateral tail vein. The recipient mice were induced with EAE either before or after the adoptive transfers.

\subsubsection{Statistical analysis for EAE suppression}

Unless stated, analysis of statistical significance of the EAE treatment between groups was performed using a one tailed Mann Whitney $U$ test and the statistical programme 
Prism (GraphPad Software Inc., San Diego, CA, USA). A p-value of less than 0.05 was considered significant.

\subsection{Histology}

Mice were sacrificed by asphyxiation with carbon dioxide $\left(\mathrm{CO}_{2}\right)$ and the spinal cords were removed by flushing the spinal column with sterile phosphate buffered saline (PBS) (Invitrogen, USA). The spinal cords were fixed in immunohistochemistry zinc fixative (BD Biosciences, Sparks, MD, USA), embedded in paraffin and sectioned longitudinally. Three serial $6 \mu \mathrm{m}$ thick sections were mounted on slides and stained with hematoxylin and eosin (H\&E) by the Pathology Department at the Wellington School of Medicine (University of Otago, New Zealand). The H\&E stained slides were analysed with an Olympus BX51 microscope and Olympus DP70 digital camera and analysis software (Olympus, Center Valley, PA, USA).

\subsection{Ex vivo techniques}

\subsubsection{Preparation of single cell suspension from lymphoid tissue}

Mice were sacrificed by $\mathrm{CO}_{2}$ asphyxiation followed by cervical dislocation. The lymph nodes, spleens and thymi were removed and placed in complete Iscoves modified Dulbecco's medium (cIMDM) (Invitrogen, USA) containing 5\% fetal calf serum (FCS) on ice (Appendix 3). A single cell suspension was prepared by dissociating tissues through a $100 \mu \mathrm{m}$ cell strainer (BD Biosciences, USA) and washing into a $50 \mathrm{ml}$ Falcon tube in cIMDM. In experiments where the spleen was processed, red blood cells were lysed by resuspending splenocytes in $5 \mathrm{ml}$ of red blood cell lysis buffer (Appendix 3) per spleen. The cells were subsequently washed twice in cIMDM by centrifuging at 600 $\mathrm{g}$ for three minutes in a Megafuge 2.0R centrifuge (Heraeus Instruments, Germany). The number of live cells was determined using a Beckman coulter Z2 cell and particle counter (Beckman Coulter Inc, Miami, FL, USA), or alternatively, by trypan blue exclusion (trypan blue stain $0.4 \%$, Invitrogen, USA) using a haemocytometer (Boeco, Germany).

\subsubsection{Preparation of single cell suspension from the blood}

Mouse blood was harvested by cardiac puncture and the blood from each mouse was mixed with $700 \mu \mathrm{l}$ of anticoagulant Alservers solution (Appendix 3) and stored on ice. Eppendorf tubes containing this blood and Alservers solution were subsequently spun in 
a microlitre centrifuge Biofuge ${ }^{\circledR}$ Fresco $^{\circledR}$ (Heraeus Instruments, Germany) for 10 minutes at $1400 \mathrm{~g}$. The supernatant containing plasma and Alsevers solution was removed and discarded after the spin. The cells were then resuspended in an appropriate solution dependent upon experimental requirements.

\subsubsection{Preparation of single cell suspension from the CNS}

Mice were sacrificed by asphyxiation with $\mathrm{CO}_{2}$ before the removal of the spinal cords and brains. The spinal cords and brains were removed and transferred to a Petri dish and were cut into small pieces using a sterile blade. The small pieces of tissue were resuspended in $5 \mathrm{ml}$ PBS containing $2.4 \mathrm{mg} / \mathrm{ml}$ collagenase type II (Invitrogen, USA). After a 30-minute incubation at $37{ }^{\circ} \mathrm{C}$, the tissue was passed through an 18-gauge needle to break up clumps and subsequently filtered through a $100 \mu \mathrm{m}$ cell strainer. After washing twice with FACS buffer, the cells were resuspended in $10 \mathrm{ml} 37 \%$ Percoll density gradient (Appendix 3) (Pharmacia Biotech, Sweden) and spun at $600 \mathrm{~g}$ for 30 minutes in a Megafuge 2.0R centrifuge (Heraeus Instruments, Germany) with the brake disengaged. This gradient separation produced a surface layer of approximately $5 \mathrm{ml}$ of volume, which contained myelin and Percoll. This layer was taken off without disturbing the cell pellet. The cell pellet together with the remaining buffer was then washed twice in cIMDM to remove traces of Percoll and resuspended in an appropriate solution dependent upon experimental requirements.

\subsubsection{Preparation of single cell suspension from the peritoneal cavity}

Mice were sacrificed by $\mathrm{CO}_{2}$ exposure. A $3 \mathrm{ml}$ solution of PBS containing $25 \mathrm{U} / \mathrm{ml}$ heparin (Hospira Australia Pty Ltd, Australia) (Appendix 3) was injected into the peritoneum near the inguinal fat pads. The inflated peritoneum was then massaged for 30 seconds. Following this massage step, the fluid was withdrawn using a syringe needle and transferred to a $50 \mathrm{ml}$ Falcon tube and kept on ice. The cells were subsequently washed and resuspended in an appropriate solution dependent upon experimental requirements.

\subsubsection{Preparation of single cell suspension from the liver}

Mice were sacrificed by $\mathrm{CO}_{2}$ exposure and perfused with $10 \mathrm{ml}$ PBS by intracardiac infusion. Livers were then removed and cut into small pieces and incubated at $37^{\circ} \mathrm{C}$ for 
one hour in $10 \mathrm{ml}$ Iscoves modified Dulbecco's medium (IMDM) containing $2.4 \mathrm{mg} / \mathrm{ml}$ collagenase type I (Invitrogen, USA) and $10 \mu \mathrm{g} / \mathrm{ml}$ DNase I (Roche Applied Science, USA). Cells were then forced through an 18-gauge needle and passed through a $100 \mu \mathrm{m}$ cell strainer to obtain single cell suspension. After being spun at $600 \mathrm{~g}$ for three minutes, the cells were resuspended in $25 \mathrm{ml}$ of room temperature pre-warmed IMDM. A $10 \mathrm{ml}$ solution of $60 \%$ Percoll was placed under the layer of cells in IMDM using a glass Pasteur pipette. After centrifugation at $600 \mathrm{~g}$ for 20 minutes at room temperature with the brake disengaged, lymphocytes located in the gradient interface were collected and diluted in IMDM. After being washed twice in IMDM to remove traces of Percoll, the cells were then resuspended in an appropriate solution dependent upon experimental requirements.

\subsection{Cell purification}

\subsubsection{Magnetic cell separation}

Single cell suspension was prepared as described in Section 2.6. Before the separation step, cells were filtered through a nylon gauze filter to remove cell clumps. Flow cytometric analysis was performed after the purification step to determine cell purity.

\subsubsection{MACS purification of $\mathrm{CD}^{+}$and $\mathrm{CD8}^{+} \mathrm{T}$ cells}

Spleens and lymph nodes were used as a source for $\mathrm{CD}^{+}$and $\mathrm{CD}^{+}$cells. Single cell suspension was prepared from spleens and lymph nodes as described in Section 2.6.1. Non-specific binding cells were depleted from the suspension by passing through an AutoMACS separator (Myltenyi Biotec, Germany) containing an AutoMACS magnetised column (Myltenyi Biotec, Germany), to which the cells non-specifically bind. The unbound fraction was then resuspended at $5 \times 10^{8}$ cells $/ \mathrm{ml}$ in AutoMACS running buffer (Appendix 3). For every $5 \times 10^{7}$ cells, $10 \mu \mathrm{CD} 4$ or CD8 beads (Miltenyi Biotec, Germany) were added. After 30 minutes incubation on a spinning wheel at $4^{\circ} \mathrm{C}$, the cells were washed twice with AutoMACS running buffer. $\mathrm{CD} 4^{+}$or $\mathrm{CD} 8^{+}$cells were then positively selected by passing through the AutoMACS column. To achieve high purity, the cells from the positive fraction were passed through the AutoMACS column again and the positive fraction was collected. After passing the positive fraction through the column for the second time, the purity could reach $>99 \%$. 


\subsubsection{MACS purification of $\mathrm{CD}^{+} \mathrm{CD}^{+} 5^{+} \mathrm{T}$ cells and $\mathrm{CD}^{+} \mathrm{CD} 25^{-} \mathrm{T}$ cells}

Mice used as a source for $\mathrm{CD} 4{ }^{+} \mathrm{CD} 25^{+}$cells were injected with $150 \mu \mathrm{g}$ of CD8 antibody (Clone 2.43) i.p. to deplete the $\mathrm{CD}^{+}$cell population. One day after the $\mathrm{CD} 8$ antibody administration, mice were sacrificed and the lymph nodes were collected. Single cell suspension of lymphocytes was prepared as described in Section 2.6.1. The subsequent purification of the two subpopulations of $\mathrm{T}$ cells $\left(\mathrm{CD} 25^{+} \mathrm{CD} 4^{+}\right.$and $\left.\mathrm{CD} 25^{-} \mathrm{CD} 4^{+}\right)$from the lymphocytic cell suspension was performed using a three-step procedure. Firstly, non-specifically binding cells were depleted by passing the single cell suspension through an AutoMACS column. The unbound fraction was collected and resuspended at $5 \times 10^{8}$ cells $/ \mathrm{ml}$ in AutoMACS running buffer. Secondly, $0.5 \mu \mathrm{g}$ CD25-PE antibody (Clone PC61, BD biosciences, USA) was added to each $800 \mu$ l cell suspension. After 15 minutes incubation on ice, the cells were washed twice in $50 \mathrm{ml}$ AutoMACS running buffer and then resuspended at $5 \times 10^{8}$ cells $/ \mathrm{ml}$ in AutoMACS running buffer. For every $5 \times 10^{7}$ cells, $5 \mu$ PE-beads (Miltenyi Biotec, Germany) were added. After 30 minutes incubation on a spinning wheel at $4^{\circ} \mathrm{C}$, the cells were washed twice with AutoMACS running buffer. After passing through the AutoMACS column, CD25 $5^{+}$cells were positively selected, leaving $\mathrm{CD} 25^{-}$and other cells in the negative fraction. Thirdly, the $\mathrm{CD} 4^{+}$cells were positively selected from the $\mathrm{CD} 25^{-}$cell fraction as described in Section 2.7.1.1.

\subsubsection{Two step purification of the blood $\mathrm{CD} 11 \mathrm{~b}^{+} \mathrm{Ly6G}^{-}$cells}

A unique two-step purification method was established in this study to isolate the blood

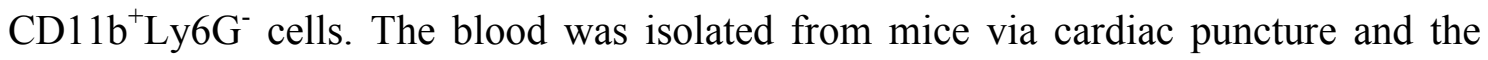
cells were processed as described in Section 2.6.2.

In the first step of cell purification, a density gradient was applied to eliminate the ${\text { Ly } 6 \mathrm{G}^{+}}^{+}$neutrophils. The cells gained from every $700 \mu \mathrm{l}$ blood were resuspended in $1.4 \mathrm{ml}$ PBS and transferred to a $15 \mathrm{ml}$ Falcon tube. The same volume of Lympholyte ${ }^{\circledR}$-Mammal cell separation medium (Cedarlane Laboratories Limited, Canada) was added to the bottom of the Falcon tubes using a glass Pasteur pipette. The tubes were then spun in a Megafuge 2.0R centrifuge (Heraeus Instruments, Germany) at $800 \mathrm{~g}$ at room temperature for 20 minutes with the brake on. After centrifugation, the interface containing blood $\mathrm{CD} 11 \mathrm{~b}^{+} \mathrm{Ly} 6 \mathrm{G}^{-}$cells was carefully removed and transferred to a new tube containing $10 \mathrm{ml}$ cIMDM. Subsequently, the cells were re-spun at $800 \mathrm{~g}$ for 
10 minutes to pellet leukocytes and the cells were resuspended at $1 \times 10^{6}$ cells $/ \mathrm{ml}$ in AutoMACS running buffer.

In the second step, a combination of antibodies with magnetic beads was utilised for $\mathrm{CD} 11 \mathrm{~b}^{+} \mathrm{Ly}_{6 \mathrm{G}}^{-}$cell purification. Before this step, the cells were passed through an AutoMACS column to get rid of non-specifically binding and dead cells. The cells were then resuspended at $5 \times 10^{8}$ cells $/ \mathrm{ml}$ in AutoMACS buffer. For every $800 \mu 1$ cell suspension, $1 \mu \mathrm{l}$ CD11b-PE antibody was added. After incubation on ice for 15 minutes, the cells were washed twice to get rid of any excess CD11b-PE antibody. The cells were then resuspended at $5 \times 10^{8}$ cells $/ \mathrm{ml}$ in AutoMACS buffer. For every $5 \times 10^{7}$ cells, $10 \mu \mathrm{l}$ anti-PE beads (Miltenyi Biotec, Germany) were added. After a 30-minute incubation while on a spinning wheel at $4^{\circ} \mathrm{C}$, cells were washed twice in AutoMACS buffer. $\mathrm{CD} 11 \mathrm{~b}^{+}$cells were positively selected by passing through a MACS column. The level of purity achieved for the CD $11 \mathrm{~b}^{+} \mathrm{Ly} 6 \mathrm{G}^{-}$cells enrichment typically exceeded $70 \%$.

\subsubsection{Depletion of $\mathrm{MHCII}^{+}$cells using BioMag beads}

Single cell suspension was prepared as described in section 2.6.1. Cells were then resuspended at $1 \times 10^{9}$ cells $/ \mathrm{ml}$ in FACS buffer (Appendix 3). Antibody against MHCII (Clone M5/114) was added to the cells at $10 \mu \mathrm{g} / \mathrm{ml}$. After incubation on ice for 15 minutes, the cells were washed twice to get rid of excess antibody and resuspended at $5 \times 10^{8}$ cells/ml in FACS buffer. The BioMag beads (Bangs Laboratories Inc., Fishers, IN, USA) were subsequently added to the cell suspension at a ratio of beads:cells $=3: 1$. After a 30-minute incubation on the spinning wheel at $4{ }^{\circ} \mathrm{C}$, cells were washed twice with FACS buffer. The tube containing the cells was placed on DynaMag-15 magnet (Invitrogen, USA) for 30 seconds and the supernatant containing the MHCII' cells was collected.

\subsubsection{Purification of $\mathrm{CD}^{+}$cells using Dynabeads}

$\mathrm{CD} 4^{+}$cells were purified using the Dynabeads FlowComp Mouse $\mathrm{CD} 4^{+}$kit (Invitrogen, USA) and DynaMag-15 magnet (Invitrogen, USA). Experimental procedures were carried out in accordance with the manufacturer's protocols. 


\subsubsection{Purification of $\mathrm{CD}^{+11 c^{+}}$cells from the spleen}

Spleens were collected from mice and placed in cold IMDM before processing. For every spleen, $2 \mathrm{ml}$ IMDM containing $1 \mathrm{mg} / \mathrm{ml}$ collagenase type II (Invitrogen, USA) and $0.2 \mathrm{mg} / \mathrm{ml}$ DNase I (Roche Applied Science, USA) was injected into the spleen. After 30 minutes incubation at $37^{\circ} \mathrm{C}$, the spleens were passed through an 18 -gauge needle to break up clumps and were filtered through a $100 \mu \mathrm{m}$ cell strainer. After being washed with FACS buffer twice, the cells were resuspended in $400 \mu$ l Automacs buffer per $10^{8}$ cells. For every $10^{8}$ cells, $25 \mu \mathrm{CD} 11 \mathrm{c}$ microbeads (Miltenyi Biotec, Germany) was added. After 30 minutes incubation on a spinning wheel at $4^{\circ} \mathrm{C}$, cells were washed twice with AutoMACS buffer. CD11 ${ }^{+}$cells were positively selected by passing through a MACS column. The level of purity achieved for the CD11 ${ }^{+}$cells typically exceeded $90 \%$.

\subsubsection{FACS sorting}

For Foxp3-GFP ${ }^{+} \mathrm{T}$ cell purification, Foxp3-GFP ${ }^{+}$cells were enriched prior to FACS sorting by depleting $\mathrm{MHCII}^{+}$cells from total lymph node cell suspension using BioMag beads (Section 2.7.1.4). For blood $\mathrm{DM}^{+}$cell purification, the cells were enriched by the two-step purification prior to FACS sorting (Section 2.7.1.3). Cells were then washed twice and resuspended at $1 \times 10^{8}$ cells $/ \mathrm{ml}$ in FACS buffer. Cell sorting was performed using the FACSDiVa cell sorter (Beckton Dickinson, USA). Unlabelled samples and single labelled controls for each fluorochrome were included for setting voltage and compensation parameters.

\subsection{Bone marrow dendritic cell culture}

Mouse bone marrow was flushed out of each femur and tibial shaft with $2 \mathrm{ml}$ cIMDM using a syringe. Clumps were broken up by resuspending with a pipette and passed through a $100 \mu \mathrm{m}$ cell strainer to remove particles. After washing the bone marrow cells twice with cIMDM, the viable cells were adjusted to a concentration of $4 \times 10^{5}$ cells $/ \mathrm{ml}$ in cIMDM containing 4\% granulocyte-macrophage colony-stimulating factor (GM-CSF, provided by Professor Franca Ronchese, Malaghan Institute, New Zealand) and $0.2 \%$ IL-4 (provided by Professor Franca Ronchese). The single cell suspension was plated at $5 \mathrm{ml} /$ well in a six-well plate. Cells were fed every two days from day three, each time by replacing $2 \mathrm{ml}$ old medium with $2 \mathrm{ml}$ fresh complete IMDM 
containing 10\% GM-CSF and 0.5\% IL-4. On day six, $100 \mathrm{ng} / \mathrm{ml}$ LPS (Sigma-Aldrich, St. Louis, MO, USA) was added to induce cell activation/maturation. Non-adherent cells were collected on day seven (routinely containing $70-80 \% \mathrm{CD} 11 \mathrm{c}^{+}$cells).

\subsection{Cytokine detection}

Cytokine levels from serum and culture supernatant were assayed using the Bio-Plex cytokine detection system (Bio-Rad, Hercules, CA, USA). L-filter plates (Linco Research In., St. Charles, MO, USA) were used for the assay. The wells were pre-wet with $100 \mu$ assay buffer (Appendix 3) before use. The sample buffer (the same buffer used in the culture supernatant) containing $0.65 \mu$ cytokine beads was added to each well. After washing the wells containing the beads twice, $50 \mu$ l samples or Bio-Plex standards (Bio-Rad, USA) were added to the wells. After incubation on an IKA-Schüttler MTS plate shaker (IKA Labortechnik, Germany) at $300 \mathrm{rpm}$ for 30 minutes at room temperature, the plate was washed three times with assay buffer. A $25 \mu \mathrm{l}$ volume of detection antibody mix made up of antibodies that had been diluted 300 times was added to each well. After a 30-minute incubation at room temperature while on a shaker at $300 \mathrm{rpm}$, the wells were washed twice with assay buffer. The secondary antibody streptavidin-PE (BD Biosciences, USA) diluted 1000 times was then added to the wells at $50 \mu \mathrm{l}$ per well. After a further 10-minute incubation at room temperature, the samples were rinsed and resuspended in $125 \mu \mathrm{l}$ assay buffer. The Bio-Plex Array Reader, Microplate plateform, and Bio-Plex Manager software (Bio-Rad, USA) were utilised for the reading of experimental results and data analysis.

\subsection{Fluorescent labelling of cells and analysis by flow cytometry}

\subsubsection{Detection of surface markers}

Single cell suspension was prepared and washed once with FACS buffer containing $0.1 \%$ sodium azide and 5\% FCS (Appendix 3) and resuspended at 0.2-1 x 10 cells $/ \mathrm{ml}$. The cell suspension was distributed to the wells of a 96-well plate in $200 \mu$ aliquots. The fluorophore conjugated antibodies that target against cell surface markers were diluted at an appropriate dilution dependent upon experimental requirements. Fc receptor blocking antibodies were added to this antibody mix at a concentration of $10 \mu \mathrm{g} / \mathrm{ml}$ (Clone 2.4G2). This antibody mix was added to each well containing cells at a volume of $50 \mu \mathrm{l}$ per well and incubated at room temperature for 10 minutes. 
Isotype-matched antibodies were used as experimental controls. Following incubation, the cells were washed twice with $200 \mu \mathrm{FACS}$ buffer. In experiments where cells were labelled using biotinylated antibodies, the cells were incubated for a further 10 minutes at room temperature with a streptavidin-conjugated fluorochrome after this wash step. Cells were resuspended in $200 \mu \mathrm{FACS}$ buffer for analysis by flow cytometry. Stained cells were gated according to forward- and side- scatter characteristics, and analysed using a FACSCalibur flow cytometer (BD Biosciences, USA). Samples with isotype-matched antibody were used as negative controls to determine proper region or window setting. Fluorescence data were analysed with FlowJo 8.8.3 (Tree Star Inc., Ashland, OR, USA) after subtraction of background isotype-matched values.

\subsubsection{Foxp3 intracellular staining}

Foxp3 intracellular staining was performed using a Foxp3 staining kit (eBioscience Inc., San Diego, CA, USA). After staining for cell surface antigens as described in Section 2.10.1, the cells were washed with cold PBS instead of FACS buffer to minimise protein binding. After this wash step, the cells were resuspended in $200 \mu \mathrm{l}$ freshly prepared fix/perm working solution provided in the kit, and incubated at room temperature for 20 minutes. After the incubation, the cells were washed once with permeabilisation buffer. The diluted anti-mouse/rat Foxp3-APC antibody (diluted 200x in permeabilisation buffer) was added to the cells and incubated at room temperature for a further 15 minutes. After incubation and being washed twice with permeabilisation buffer, the cells were resuspended in FACS buffer and analysed by flow cytometry.

\subsubsection{CFSE labelling of cells}

Carboxyfluorescein diacetate succinimidyl ester (CFSE) labelling has been used as a routine procedure for the analysis of cell division both in vivo and in vitro ${ }^{188}$. This method relies on the labelling of long-lived intracellular molecules with carboxyfluorescein. Following each cell division, the equal distribution of these fluorescent molecules between daughter cells allows cell proliferation to be tracked $^{188}$.

Single cell suspension was prepared as described in Section 2.6.1. Cells were resuspended in $20 \mathrm{ml}$ HBSS containing $2 \mathrm{mM}$ ethylenediaminetetraacetic acid (EDTA) (Invitrogen, USA). The cell numbers in each tube did not exceed $4 \times 10^{8}$ cells. Cells were 
subsequently labelled with $200 \mathrm{nM}$ CFSE (Invitrogen, USA). After approximately a seven-minute incubation at room temperature, the tube was filled up with cIMDM containing 5\% FCS and spun at $800 \mathrm{~g}$ for five minutes. After being washed twice, the cells were then resuspended in an appropriate solution dependent upon experimental requirements. The labelling of cells by CFSE was analysed by flow cytometry.

\subsubsection{Detecting apoptotic and necrotic cells}

Annexin V-FITC (an early apoptotic marker) (BD Biosciences, USA) in combination with the vital dye propidium iodidie (PI) (Sigma-Aldrich, USA) was used to identify viable cells. Annexin V is a calcium dependent phospholipid binding protein that has a high affinity for phosphatidylserine ${ }^{189}$. Externalisation of phosphatidylserine from the inner leaflet of the plasma membrane to the outer leaflet is associated with cellular apoptosis $^{189}$. PI binds to DNA in the nucleus when the cell membrane has lost its integrity and become permeable. Double negative populations indicate viable cells, while annexin-V single positives indicate early apoptotic cells. PI single positives are necrotic cells while annexin-V/PI double positives are late stage dying and dead cells.

The cells were washed twice with excess chilled Annexin V buffer (Appendix 3) and incubated in the dark at $4^{\circ} \mathrm{C}$ for 15 minutes with $1 \mu$ Annexin V-FITC antibody (BD Biosciences, USA) diluted with $100 \mu$ Annexin V buffer. After incubation, these cells were washed twice with Annexin V buffer. Just prior to FACS analysis, PI was diluted 1:8000 times and added to the samples.

\subsubsection{Intracellular cytokine staining}

Prior to intracellular labelling of cytokines, cells were resuspended in 24-well plates at $1-5 \times 10^{6}$ cells $/ \mathrm{ml}$ in cIMDM and stimulated with stimuli e.g. phorbol 12-myristate 13-acetate (PMA) (Invitrogen, USA) and ionomycine (Sigma-Aldrich, USA). Monensin (Calbiochem, San Diego, CA, USA) was added to the cells from the beginning of the cell culture at $2 \mu \mathrm{M}$ to block intracellular protein transport. Cells were incubated at $37^{\circ} \mathrm{C}$ for five hours.

After in vitro stimulation, these cells were labelled with antibodies for cell surface 
markers as described in Section 2.11.1. Subsequently, the cells were fixed and permeabilised using a BD Cytofix/Cytoperm Kit (BD Biosciences, USA). Briefly, the cells were washed with FACS buffer and fixed for 20 minutes by incubating with $200 \mu 1$ BD Cytofix/Cytoperm solution at room temperature. The cells were then washed twice with BD Perm/Wash buffer. Antibodies for cytokines or the respective isotype control were added to $50 \mu \mathrm{FACS}$ buffer and incubated with the cells for 30 minutes on ice. Following the incubation, the cells were washed twice more with cold BD Perm/Wash buffer and subsequently resuspended in $200 \mu$ FACS buffer and kept at $4^{\circ} \mathrm{C}$ before analysis by flow cytometry

\subsection{In vitro cell functional assays}

\subsubsection{Proliferation assay}

Cells were plated in triplicates in a $96-$ well plate at $2 \times 10^{5}$ to $1 \times 10^{6}$ cells $/ \mathrm{ml}$ plus appropriate peptides or antibodies in $200 \mu \mathrm{l}$ cIMDM dependent on the individual experiment. For plate-bound anti-CD3/anti-CD28 proliferation assays, the 96-well plates were coated with anti-CD3 and anti-CD28 antibodies (concentration is indicated in individual experiment) in $\mathrm{PBS}$ overnight at $37^{\circ} \mathrm{C}$. Before adding cells, the plate was washed three times using PBS. Cells were incubated in the plate for 72 hours. After 72 hours, $0.25 \mu \mathrm{Ci}\left[{ }^{3} \mathrm{H}\right]$-thymidine (GE Healthcare, UK) was added to each well for additional 8-16 hours. The cells were harvested to filtermats (PerkinElmer life sciences, Finland) using an automated cell harvester (Tomtec Inc., Hamden, CT. USA) and read on a Topcount Microplate scintillation counter (Wallac, Woodbridge, ON, USA).

\subsubsection{Suppression assay}

The suppression of $\mathrm{T}$ cell proliferation by suppressor cells was evaluated using either $\left[{ }^{3} \mathrm{H}\right]$-thymidine uptake or CFSE dilution based assay.

\subsubsection{1 $\left[{ }^{3} \mathrm{H}\right]$-thymidine uptake based suppression assay}

Purified suppressor cells such as the blood CD11 $\mathrm{b}^{+} \mathrm{Ly}_{6 \mathrm{G}^{-}}$cells or Tregs were plated in triplicates in a 96-well round-bottom plate. The suppressor and responder cells were cultured together at ratios of 1:1,1:2, 1:4 and 1:8 with the same numbers of responder cells in each well. Cells were stimulated by different stimuli and incubated at $37^{\circ} \mathrm{C}$ for different time periods dependent upon experimental requirements. The total volume of 
medium in each well was adjusted to $175 \mu \mathrm{l}$, and $0.25 \mu \mathrm{Ci}$ of $\left[{ }^{3} \mathrm{H}\right]$-thymidine (GE Healthcare, UK) was added to each well and incubated for an additional 8-16 hours. The cells were harvested to filtermats (PerkinElmer Life Sciences, Finland) using an automated cell harvester (Tomtec Inc., USA) and read on a Topcount Microplate scintillation counter (Wallac, USA).

Suppression was calculated using the following formula:

$\left[1-\mathrm{cpm}(\right.$ target cells with suppressor cells)/cpm(target cells alone) $] \times 100 \%{ }^{190}$

\subsubsection{CFSE dilution based suppression assay}

The responder cells were labelled with CFSE as described in Section 2.10.3. The mixture of CFSE labelled responder cells and suppressor cells were plated in triplicates in a 96-well round-bottom plate. The suppressor and responder cells were cultured together at ratios of 1:1,1:2,1:4 and 1:8 with the same number of responder cells in each well. Cells were stimulated using different stimuli according to experimental requirements. The total volume of medium in each well was adjusted to $175 \mu \mathrm{l}$. Cells were incubated at $37^{\circ} \mathrm{C}$ for 72 hours, and the cell proliferation was measured by CFSE dilution using flow cytometry.

\subsubsection{Statistical analysis for cell proliferation and suppression}

Unless stated, analysis of statistical significance of cell proliferation and suppression was performed using One-way analysis of variance (ANOVA) followed by Bonferroni tests and the statistical program Prism (GraphPad Software Inc., USA). A p-value of less than 0.05 was considered significant.

\subsection{Morphological stain for cells}

Cells were spun onto a slide (LabServ, Australia) with a Shandon Cytospin 4 cytocentrifuge (Thermo Fisher Scientific, USA) at $800 \mathrm{~g}$ for five minutes and then were allowed to air dry. A Diff-Quik stain set (Dade behring Inc, Deerfield, IL. USA) was used to visualise cell morphology. Slides were fixed for five seconds in Diff-Quik fixative $(1.8 \mathrm{mg} / \mathrm{l}$ Triarylmethane dye methyl alcohol), stained for 10 seconds in Diff-Quik Solution I (1 g/l Xanthine dye) and further stained for seven seconds using 
Diff-Quik Solution II (0.625 g/l Azure A, $0.625 \mathrm{~g} / 1$ methylene blue). Excess dye was then washed off with water. Slides were viewed using light microscopy (Olympus, Japan).

\subsection{Confocal microscopy}

Cells on a slide were visualised using a Leica TCS SP2 confocal microscope (Leica Microsystems, Germany). Images were acquired at room temperature using a photomultiplier tube and Leica Confocal software. The acquired images were then processed using NIH ImageJ image manipulation software (NIH, Maryland, USA). Figures were generated using Adobe Photoshop software (Adobe, San Jose, CA, USA).

\subsection{Biochemistry}

\subsubsection{Gel electrophoresis}

Non-reducing sodium dodecyl sulfate polyacrylamide gel electrophoresis (SDS PAGE) was used to determine protein sizes and evaluate the efficiency of peptides coupling to mSAgs. Aliquots containing proteins or peptides were mixed with NuPAGE LDS sample buffer (Invitrogen, USA) and added to the stacking wells of a NuPAGE Novex Bis-Tris 10-well gel (Invitrogen, USA). A Precision Plus Protein prestained standard (Bio-Rad) was added to one well of the gel for approximate molecular weight determination. The gels were run using an XCell SureLock ${ }^{\mathrm{TM}}$ Mini-Cell (Invitrogen, USA) and a PowerPac 3000 power supply (Bio-Rad, USA) at a constant voltage of $200 \mathrm{~V}$ for 40 minutes in NuPAGE MES SDS running buffer (Invitrogen, USA). The gels were subsequently stained with Coomassie blue solution (Appendix 3) for one hour with constant shaking and incubated in the destain solution (Appendix 3) at room temperature overnight. Gel pictures were taken by a Molecular Imager Gel Doc XR documentation system (BioRad, USA), and analysed by the Quantity One version 4.6.5 software (BioRad, USA).

\subsubsection{Bradford Assay}

The Bradford assay was used to determine protein concentrations. The protein was diluted to an estimated concentration of $1-20 \mathrm{mg} / \mathrm{ml}$. Standards containing a range of 1-20 $\mu$ protein e.g. IgG were prepared to a volume of $200 \mu \mathrm{l}$ in the same buffer. Samples $(100 \mu \mathrm{l})$ were incubated with $100 \mu \mathrm{l}$ Bradford reagent (Appendix 3) at room 
temperature for five minutes. The absorbance was measured at $595 \mathrm{~nm}$ using a BioRad Benchmark microplate reader (Bio-Rad, USA). A standard curve was drawn for the protein standard to allow the calculation of the protein concentration in the samples.

\subsubsection{Labelling proteins by using fluorophore or biotin}

The protein for labelling was concentrated in $0.1 \mathrm{M}$ sodium bicarbonate buffer to $0.1 \mathrm{mM}$ by using a $10 \mathrm{KDa}$ cut-off vivaspin 500 (Sartorius, Germany). EZ-Link NHS-Chromogenic-Biotin (Pierce, USA), Alexa 488 or Alexa 647 dye (Invitrogen, USA) were stored in DMSO (Sigma-Aldrich, USA) at $10 \mathrm{mg} / \mathrm{ml}$ at $-80^{\circ} \mathrm{C}$. For every $4 \mathrm{nmol}$ protein, $10 \mu \mathrm{g}$ biotin or Alexa dye was added to the protein solution while vortexing. After 10 minutes incubation at room temperature, the excess dye was removed by spinning the protein solution through a $10 \mathrm{KDa}$ cut-off vivaspin column. The labelled protein was tested by FACS staining and stored in PBS containing $0.1 \%$ sodium azide.

\subsubsection{Griess reaction}

A Griess reaction was used to detect $\mathrm{NO}_{2}$ - for measuring $\mathrm{NO}$ formation. The supernatant $(50 \mu \mathrm{l})$ for testing was plated in a flat bottom 96-well plate. $\mathrm{NaNO}_{2}$ (Promega Corporation, Madison, WI, USA) was titrated to perform a standard curve (starting from $500 \mu \mathrm{M}$ ). Equally mixed Griess solutions A and B (Appendix 3) of $50 \mu \mathrm{l}$ were added to each well. After incubation at room temperature for 20 minutes, the plate was read using an absorbance of $570 \mathrm{~nm}$ on a VersaMax Tunable Microplate Reader (Molecular Devices Pty Ltd, Australia). A standard curve was constructed for the $\mathrm{NaNO}_{2}$ standard to allow calculation of the $\mathrm{NO}_{2}$ - concentration in the samples. 


\section{Chapter 3:}

\section{SM-MOG $_{35-55}$ Mediated EAE Suppression}

In part published in Dickgreber, N., Stoitzner, P., Bai, Y., Price, K.M., Farrand, K.J., Manning, K., Angel, C.E., Dunbar, P.R., Ronchese, F., Fraser, J.D., Bäckström, B.T. and Hermans, I.F. Targeting antigen to MHC class II molecules promotes efficient cross-presentation and enhances immunotherapy. J Immunol 182, 1260-1269 (2009).

Maiden name Yan Bai. Changed to Clare Yan Slaney in 2009. 


\subsection{Introduction}

Bacterial superantigens are a family of structurally related protein toxins considered to be the most powerful $\mathrm{T}$ cell mitogens discovered to date ${ }^{191}$. Traditional peptide antigens presented via MHCII activate $0.001-0.0001 \%$ of the total $\mathrm{T}$ cell repertoire ${ }^{192}$. In contrast, superantigens can activate $5-20 \%$ of the $\mathrm{T}$ cell repertoire by binding simultaneously to MHCII on APCs and TCRs on T cells, which leads to a massive immune response and serious human pathologies such as toxic shock syndrome ${ }^{173}$.

SMEZ-2-M1 (SM) provided by Professor John Fraser (the University of Auckland, New Zealand) is a mutant form of superantigen SMEZ-2 and lacks TCR binding ability. SM contains three alterations in the TCR binding site, W75L, K182Q and D42C. The mutations W75L and K182Q prevent the binding of SM to TCRs, and D42C mutation introduces an exposed cysteine for direct conjugation of antigens ${ }^{193}$. The deficit of SM's binding to TCR eliminates SM's toxicity and therefore, SM can be used as a potential non-toxic antigen-delivery vehicle for targeting $\mathrm{MHCII}^{+}$APCs.

A previous study in our laboratory showed that when C57BL/6J mice were treated with $1 \mu \mathrm{g}$ of the $\mathrm{SM}$ conjugate with the $\mathrm{MOG}_{35-55}$ peptide $\left(\mathrm{SM}_{-} \mathrm{MOG}_{35-55}\right)$ in IFA s.c. five to seven days after EAE induction, the disease was abrogated ${ }^{168}$. Experimental evidence suggested that $\mathrm{MOG}_{35-55}$-specific Tregs played a role in $\mathrm{SM}-\mathrm{MOG}_{35-55}$-mediated EAE suppression. However, the precise mechanism involved was not determined. Based on these findings, I hypothesised that $\mathrm{SM}^{-\mathrm{MOG}_{35-55}}$ expanded and/or activated a population of $\mathrm{MOG}_{35-55}$-specific Tregs in vivo, which inhibited the self-reactive $\mathrm{MOG}_{35-55}$-specific $\mathrm{CD}^{+} \mathrm{T}$ cell response in mice induced with EAE.

\subsection{Aims}

The overall aim of this chapter was to investigate the role of $\mathrm{MOG}_{35-55}$ specific Tregs in the $\mathrm{SM}-\mathrm{MOG}_{35-55}$ mediated suppression of EAE and to understand the cellular and molecular changes during the $\mathrm{SM}-\mathrm{MOG}_{35-55}$ mediated immune suppression in vivo.

The specific aims were as follows:

- To optimise the conjugation of peptides to SM

- To understand which subpopulation of Tregs was important for the

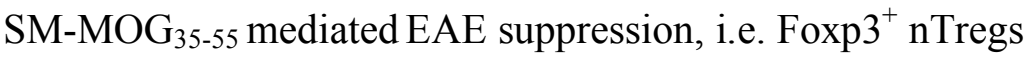


- To optimise the $\mathrm{SM}-\mathrm{MOG}_{35-55}$ treatment of EAE by optimising the

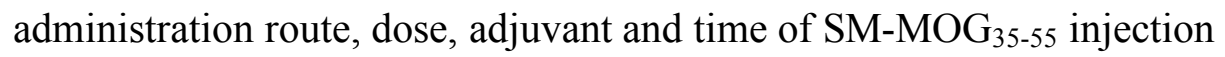

- To investigate whether there was any other suppressive cell population involved in the $\mathrm{SM}-\mathrm{MOG}_{35-55}$ mediated EAE suppression

\subsection{Results}

\subsubsection{Conjugation of peptides to SM}

To investigate the effect of SM-MOG $35-55$ on EAE, it was first necessary to prepare the

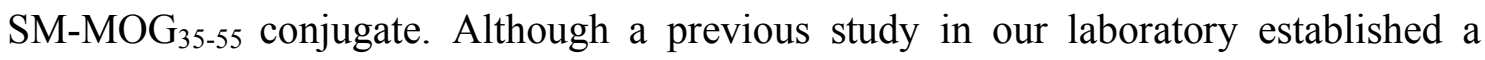
protocol for the conjugation of peptides to $\mathrm{SM}^{168}$, pilot conjugate experiments in the current study showed that the efficiency of the conjugation was poor. Therefore, a variety of experimental conditions were tested in order to optimise the conjugation of peptides to SM.

\subsubsection{Peptides could be efficiently conjugated to SM}

A summary of the experimental conditions tested to improve the efficiency of SM-peptide conjugation is presented in Table 3.1. As shown in Figure 3.1, the $\mathrm{MOG}_{35-55 \mathrm{c}}$ and $\mathrm{OVA}_{323-339 \mathrm{c}}$ peptides could be efficiently conjugated to $\mathrm{SM}$ and the excess peptides could be removed from the conjugates. The detailed optimised

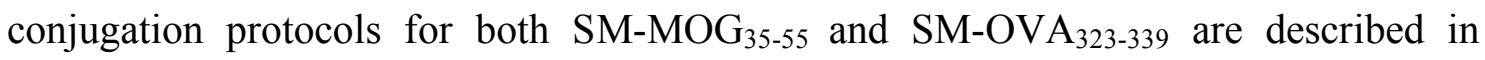
Section 2.3.1 and Section 2.3.2.

Table 3.1 Optimisation of the SM-peptide conjugation

\begin{tabular}{|c|c|c|}
\hline & Modified superantigen & Peptides \\
\hline Reducing agent & $\begin{array}{l}5 \mathrm{mM} \text { TCEP (Pierce, USA) } \\
0.01 \mathrm{M} \text { DTT (Sigma, USA) }\end{array}$ & $\begin{array}{l}5 \mathrm{mM} \text { TCEP (Pierce, USA) } \\
0.01 \text { M DTT (Sigma, USA) }\end{array}$ \\
\hline $\begin{array}{l}\text { Columns for removing } \\
\text { the reducing agent }\end{array}$ & $\begin{array}{l}\text { Vivaspin } 500 \\
\text { Polyethersulfone } 10 \text { KDa cut- } \\
\text { off (Sartorius, Germany) }\end{array}$ & $\begin{array}{l}\text { D-Salt Polyacrylamide } \\
\text { Desalting column, } 1.8 \mathrm{KDa} \\
\text { cut-off (Pierce, USA) }\end{array}$ \\
\hline $\begin{array}{l}\text { Conditions for cysteine- } \\
\text { cysteine binding }\end{array}$ & \multicolumn{2}{|c|}{$\begin{array}{l}1: 3,1: 5,1: 10 \text { and } 1: 20 \text { (molar SM:peptides) } \\
\pm \text { coupling buffer at } \mathrm{pH}=6,7,8,9 \text { and } 10\end{array}$} \\
\hline Other linkers tested & \multicolumn{2}{|c|}{$\begin{array}{l}\text { GMBS (Pierce, USA), MTFB and S-C6-HyNic (SoluLink, } \\
\text { USA) }\end{array}$} \\
\hline $\begin{array}{l}\text { Columns for the } \\
\text { Purification of } \\
\text { conjugates }\end{array}$ & \multicolumn{2}{|c|}{$\begin{array}{l}\text { Vivaspin } 500 \text { Polyethersulfone } 10 \text { KDa cut-off (Sartorius, } \\
\text { Germany), Sephacryl S-200 column (Sigma, USA), Zeba } \\
\text { Desalt Spin Column (Pierce, USA), AutoMACS separator } \\
\text { and autoMACS columns, LS columns and VarioMACS } \\
\text { separator (Miltenyi Biotec, Germany) }\end{array}$} \\
\hline
\end{tabular}




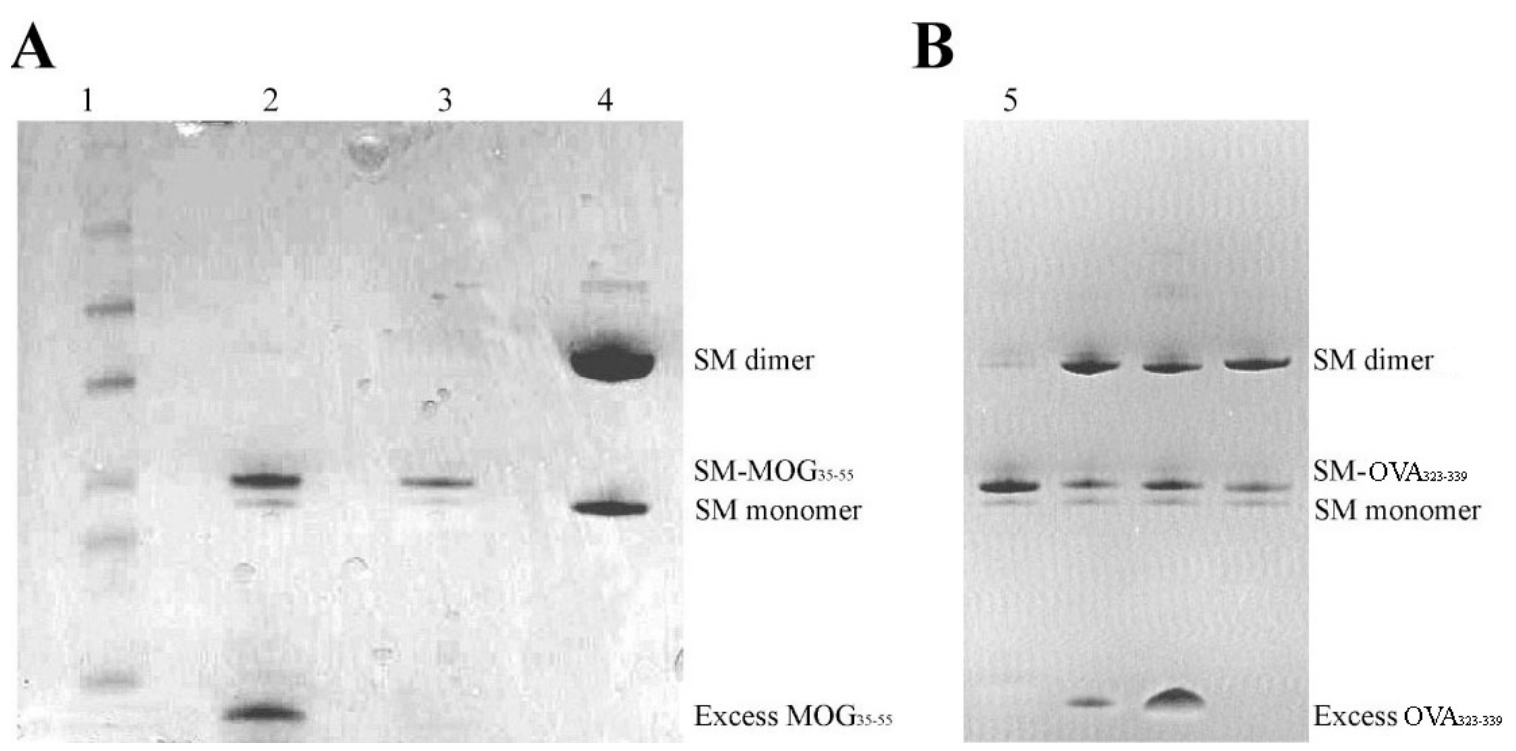

Figure 3.1 The MOG $_{35-55 c}$ and $O V_{323-339 c}$ peptides could be efficiently conjugated to $\mathrm{SM}$

A, SM-MOG $35-55$ conjugation. Lane 1, molecular weight marker; lane $2, \mathrm{MOG}_{35-55 \mathrm{c}}$ was conjugated to $\mathrm{SM}$ and lane 3, the excess $\mathrm{MOG}_{35-55 \mathrm{c}}$ peptide was removed from the $\mathrm{SM}-\mathrm{MOG}_{35-55}$ conjugate; lane 4, uncoupled SM. B, SM-OVA $323-339$ conjugation. The samples on different lanes were from different

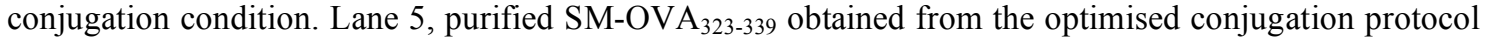
that was used in this study. The rest of the lanes on $\mathbf{B}$ are the conjugates derived from other non-optimised conjugation condition.

\subsubsection{Conjugation of peptide to SM improved efficiency of the peptide presentation to $\mathrm{CD}^{+} \mathrm{T}$ cells in vitro}

Next I tested whether the newly conjugated SM-peptides could efficiently deliver peptides to APCs and enhance the peptide-specific $\mathrm{CD}^{+} \mathrm{T}$ cell proliferation. Splenocytes were isolated from TCR transgenic mice specific for either $\mathrm{MOG}_{35-55}$ (2D2 mice) or $\mathrm{OVA}_{323-339}$ (OTII mice) and incubated with either SM-peptide conjugates or the free peptides. As shown in Figure 3.2, SM-peptide conjugates were 100-1,000 times more potent than the unconjugated peptides at activating TCR transgenic splenocyte proliferation. In contrast, the unconjugated SM did not induce splenocyte proliferation, indicating that the proliferative effect of SM-peptides was dependent on the peptides conjugated to SM. OT-II T cells were repeatedly observed to respond more potently to both peptide and the SM-peptide conjugates than 2D2 T cells. This difference may be explained by the inherent difference between the two mouse strains. 

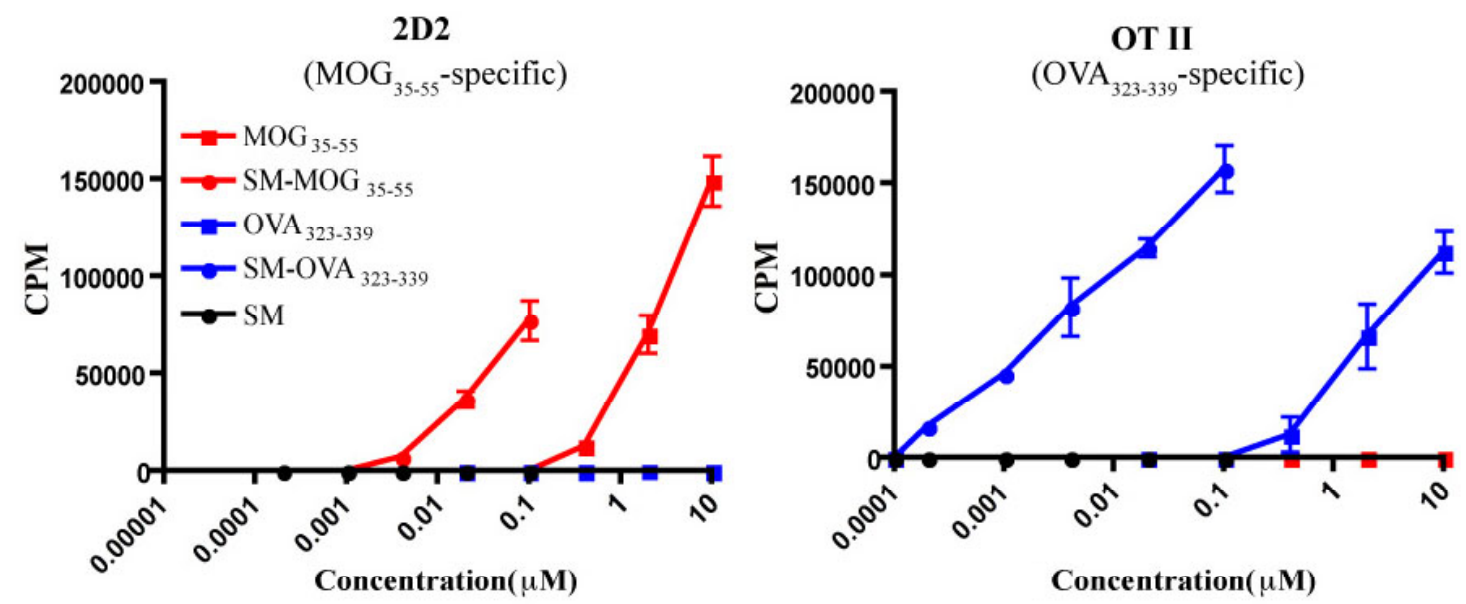

Figure 3.2 The peptides conjugated to $\mathrm{SM}$ were efficiently presented to $\mathrm{CD}^{+} \mathrm{T}$ cells in vitro

The splenocytes $\left(1 \times 10^{6}\right.$ cells/well $)$ from either $2 \mathrm{D} 2$ or OTII transgenic mice were incubated with the indicated concentrations of antigens. Cell proliferation was assayed by measuring the incorporation of $\left[{ }^{3} \mathrm{H}\right]$-thymidine over the last 16 hours of a 72 -hour culture period. Results are shown as the means $\pm \mathrm{SEM}$ of triplicate wells. Results are representative of more than three separate experiments.

\subsubsection{Conjugation of peptide to SM improved efficiency of the peptide presentation to $\mathrm{CD4}^{+} \mathrm{T}$ cells in vivo}

I then investigated whether the conjugation of peptides to SM would improve peptide presentation and proliferation of $\mathrm{CD}^{+} \mathrm{T}$ cells in vivo. First, CFSE labelled OTII or 2D2 splenocytes (CD45.2 $2^{+}$) were adoptively transferred into congenic CD45. $1^{+}$recipients $\left(5 \times 10^{6}\right.$ cells per mouse). One day later, SM-peptides or 10 times molar equivalent of the unconjugated peptides were injected i.v. into the recipient mice. Cells from the spleens and lymph nodes of the recipients were isolated at day five and analysed by flow cytometry to identify $\mathrm{CFSE}^{+} \mathrm{CD} 45.2^{+}$TCR-specific donor cells. Proliferation of $\mathrm{CD} 45.2^{+} \mathrm{CD}^{+} \mathrm{T}$ cells is represented by progressive halving of cellular CFSE fluorescence with every cell division completed. As shown in Figure 3.3, the proliferation of the peptide-specific $\mathrm{CD}^{+} \mathrm{T}$ cells was only induced following the administration of SM-peptides, indicating that peptides conjugated to SM were presented more efficiently to $\mathrm{CD}^{+} \mathrm{T}$ cells in vivo compared to the unconjugated peptides.

Consistent with the in vitro data shown in Figure 3.2 and experiments done in our laboratory, OT-II T cells responded much more potently to the SM-peptide conjugate than 2D2 T cells in vivo. Given that MOG, but not OVA is a self-antigen, it is expected 
that $\mathrm{OVA}_{323-339}$-specific $\mathrm{T}$ cells would express a TCR with a higher affinity and therefore, respond to a lower concentration of peptide.

A
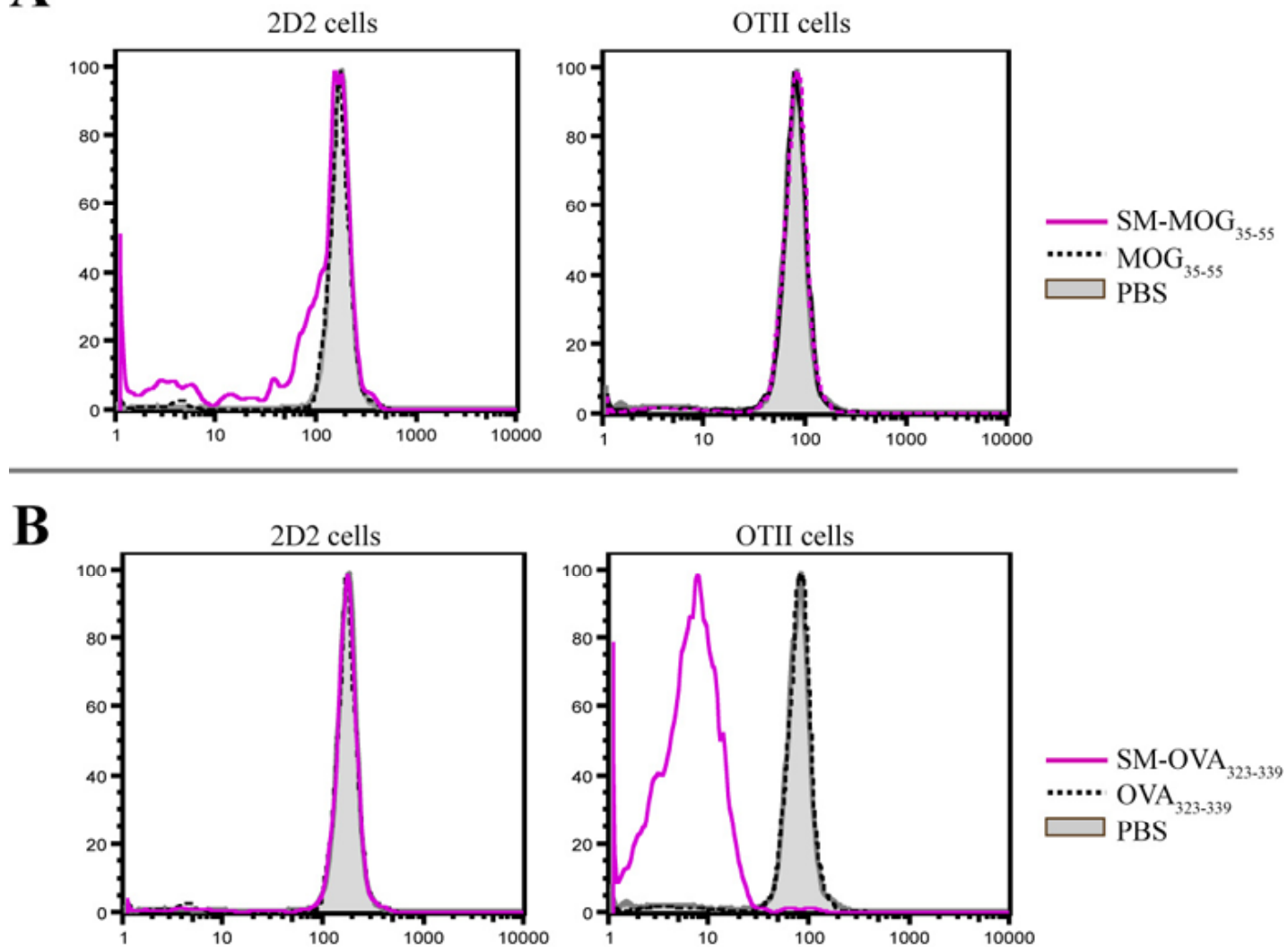

Figure 3.3 The peptides conjugated to SM were efficiently presented to $\mathrm{CD}^{+} \mathrm{T}$ cells in vivo

CFSE labelled OTII or 2D2 splenocytes were respectively transferred into congenic CD45. $1^{+}$recipient as indicated $\left(5 \times 10^{6}\right.$ cells per mouse). On day one, the recipient mice were injected i.v. with one of the following: $\mathbf{A}, 2 \mu \mathrm{g}$ of $\mathrm{SM}-\mathrm{MOG}_{35-55}, 2 \mu \mathrm{g}$ of $\mathrm{MOG}_{35-55}\left(\sim 10\right.$ times excess molar dose of $\left.\mathrm{MOG}_{35-55}\right)$ or PBS. B, $2 \mu \mathrm{g}$ of SM-OVA $323-339,2 \mu \mathrm{g}$ of $\mathrm{OVA}_{323-339}\left(\sim 10\right.$ times excess molar dose of $\left.\mathrm{OVA}_{323-339}\right)$ or PBS. Proliferation of the CFSE-labelled $\mathrm{CD}^{+} \mathrm{T}$ cells was assessed by flow cytometry of lymph node on day five. Cells were gated on $\mathrm{CD} 45.2^{+} \mathrm{CD} 4^{+}$cells.

\subsubsection{SM-MOG $35-55 /$ IFA treatment did not suppress EAE}

After confirming that the newly conjugated $\mathrm{SM}-\mathrm{MOG}_{35-55}$ was able to induce $\mathrm{T}$ cell proliferation both in vitro and in vivo, I set out to determine whether this $\mathrm{SM}-\mathrm{MOG}_{35-55}$ could suppress EAE via the CD25 ${ }^{+}$Tregs as reported in the previous study ${ }^{168}$.

\subsubsection{SM-MOG S5-55 $_{\text {IFA treatment did not suppress EAE via Foxp3 }}{ }^{+}$Tregs}

To determine whether $\mathrm{SM}_{-\mathrm{MOG}_{35-55}} / \mathrm{IFA}$ treatment suppressed EAE via Foxp ${ }^{+}$nTregs, I performed an adoptive transfer experiment using the Foxp3-GFP knock-in (KI) mice. The Foxp3-GFP KI mice harbour a GFP-Foxp3 fusion protein reporter knock-in 
allele ${ }^{182}$ and therefore, the Foxp $3^{+}$Tregs can be identified by their GFP expression. As $\mathrm{SM}_{-\mathrm{MOG}_{35-55} \text { was }} 100$ times more potent than $\mathrm{MOG}_{35-55}$ to elicit $\mathrm{T}$ cell expansion in vitro (Figure 3.2), Foxp3-GFP KI mice were treated with either $20 \mu \mathrm{g}$ of $\mathrm{MOG}_{35-55} / \mathrm{IFA}$

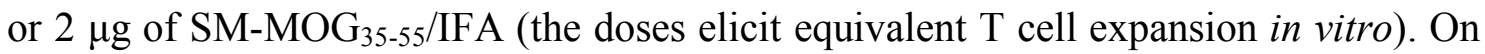
day five, the Foxp3-GFP ${ }^{+}$cells were purified by FACS sorting from either $\mathrm{MOG}_{35-55}$ or SM-MOG $35-55$ treated mouse draining lymph nodes (DLNs) and adoptively transferred into littermate recipient mice $\left(2 \times 10^{5} \mathrm{Foxp}^{+}\right.$cells per mouse). Six days after the adoptive transfer, EAE was induced in the recipient mice. This experimental set-up was similar to the previous study, except that the previous study transferred CD $25^{+} \mathrm{T}$ cells into $\mathrm{C} 57 \mathrm{BL} / 6 \mathrm{~J}$ mice.

As shown in Figure 3.4, adoptive transfer of the Foxp3 ${ }^{+}$cells from both $\mathrm{SM}-\mathrm{MOG}_{35-55}$ and $\mathrm{MOG}_{35-55}$ treated mice failed to protect the recipient mice from EAE. This raised the question of whether the lack of EAE suppression was associated with the inability

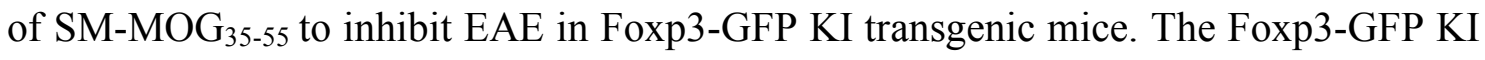
transgenic mice utilised in this study had been backcrossed with C57BL/6J mice for more than 10 generations. However, it was possible that genetic material remnant of the original Foxp3-GFP KI mouse strain could be responsible for the failure of the

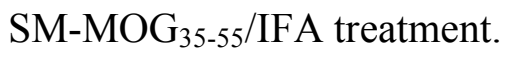
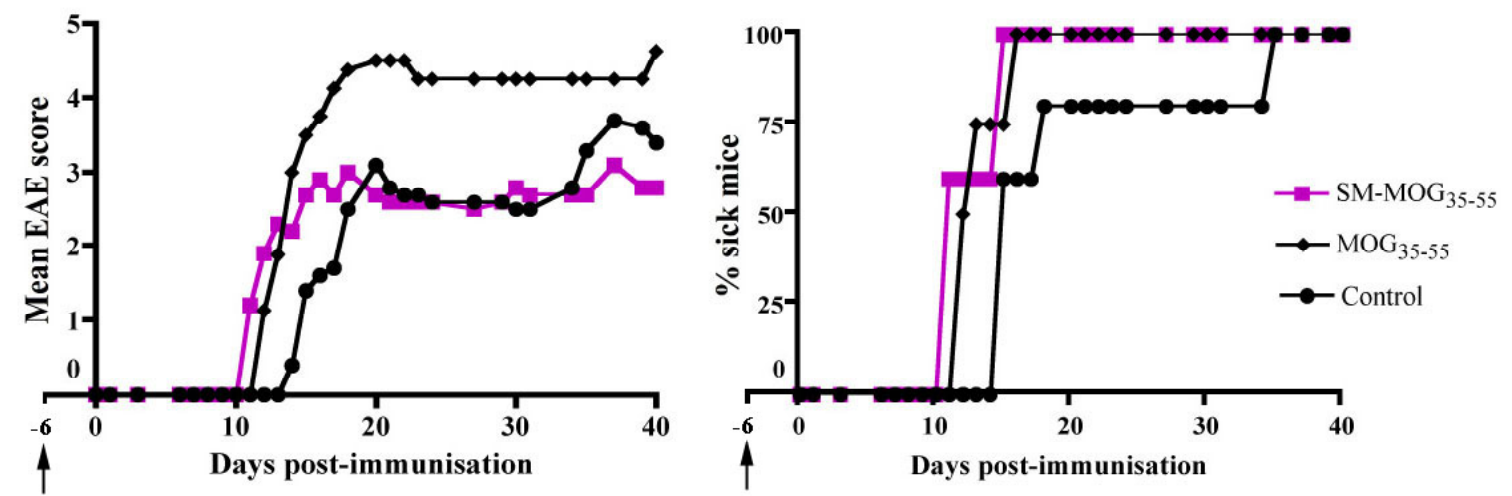

Figure 3.4 Adoptive transfer of the $\mathrm{Foxp}^{+}$cells from $\mathrm{SM}^{+} \mathrm{MOG}_{35-55} / \mathrm{IFA}$ treated mice did not suppress $\mathrm{EAE}$ in the recipient mice

The Foxp3-GFP KI mice were treated with either $20 \mu \mathrm{g}$ of $\mathrm{MOG}_{35-55} / \mathrm{IFA}$ or $2 \mu \mathrm{g}$ of SM-MOG $\mathrm{MO}_{35-55} / \mathrm{IFA}$. On day five, $2 \times 10^{5}$ purified Foxp $3^{+}$cells isolated from the DLNs of the treated mice were adoptively transferred into littermate recipient mice. Age- and sex- matched Foxp3-GFP KI mice were injected with $200 \mu \mathrm{l}$ PBS i.v. as the experimental controls. Six days after the adoptive transfer, the recipient mice were induced with EAE. The $\mathrm{MOG}_{35-55}$ group contained four mice and the other two groups contained five mice each. Left graph, mean clinical scores. Right graph, \% sick mice. Arrows indicate the day of Foxp3-GFP ${ }^{+}$cell adoptive transfer. 


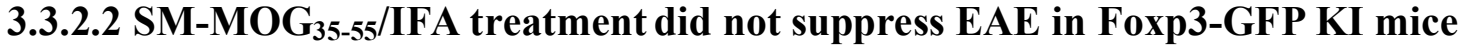

To address the question whether the $\mathrm{SM}-\mathrm{MOG}_{35-55} / \mathrm{IFA}$ treatment could suppress EAE in Foxp3-GFP KI mice, I induced EAE in Foxp3-GFP KI mice. On day five, these mice

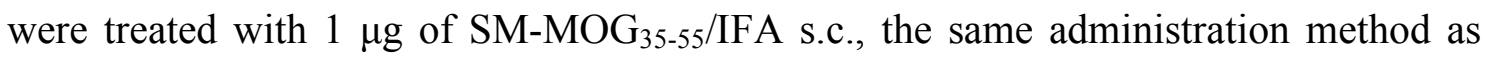
used in the previous study ${ }^{168}$. The Foxp3-GFP KI mice treated with SM-OVA $323-339 /$ IFA were used as the experimental controls. As shown in Figure 3.5, the mice treated with

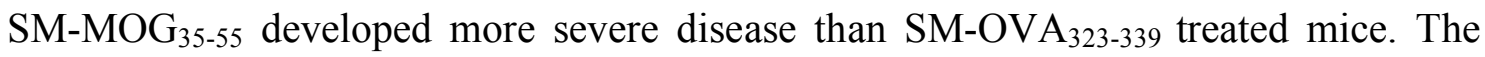
reason for the more severe disease may be due to experimental variability. Nevertheless, the treatment using SM-MOG $35-55 /$ IFA did not protect Foxp3-GFP KI mice from EAE. Therefore, the failure of EAE suppression by adoptive transfer of

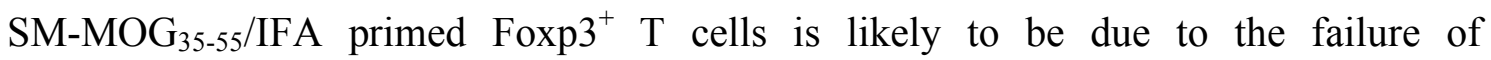
SM-MOG $35-55 /$ IFA to suppress EAE in Foxp3-GFP KI mice.
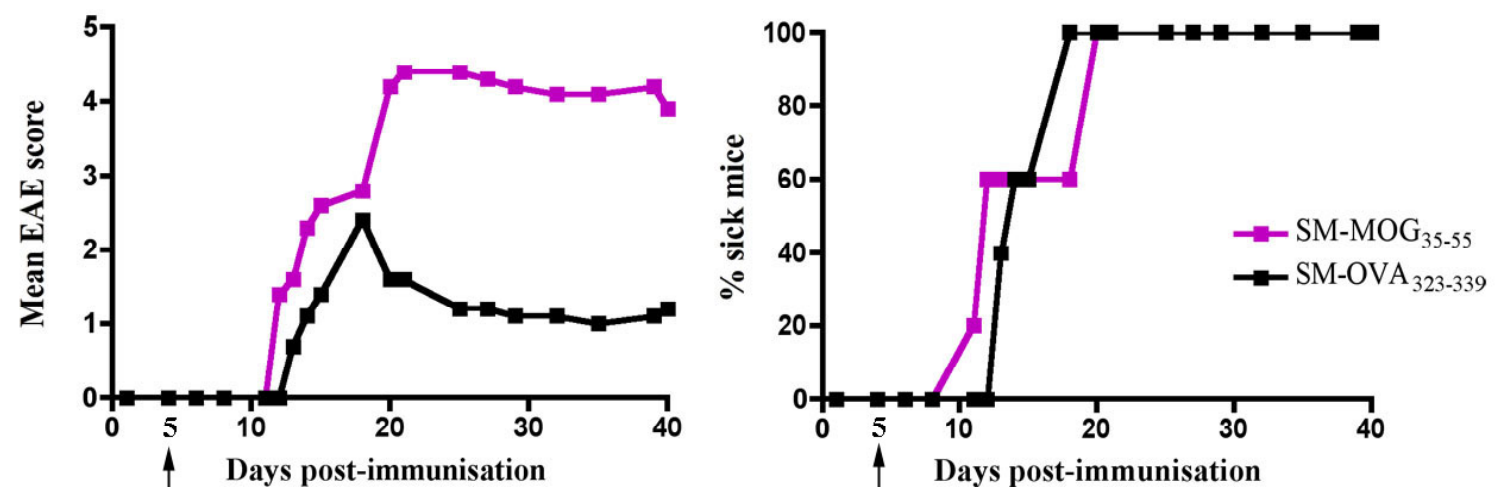

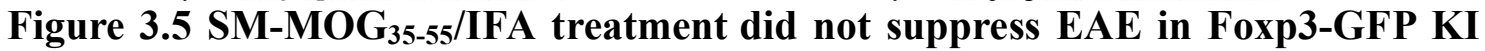
mice

Foxp3-GFP KI mice were induced with EAE. Five days after EAE induction, these mice were treated with either $1 \mu \mathrm{g}$ SM-MOG $35-55$ or $1 \mu \mathrm{g}$ SM-OVA $323-339$ in IFA s.c. Each group contained five mice. Left graph, mean EAE scores. Right graph, \% sick mice. Arrows indicate the day of SM-MOG $\mathrm{MO}_{35-55}$ or SM-OVA $323-339$ injection.

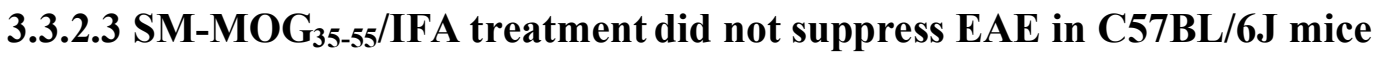

To determine whether the lack of EAE suppression by $\mathrm{SM}-\mathrm{MOG}_{35-55} / \mathrm{IFA}$ was specific to the Foxp3-GFP KI mouse strain or failure of the conjugate treatment, I tested if the

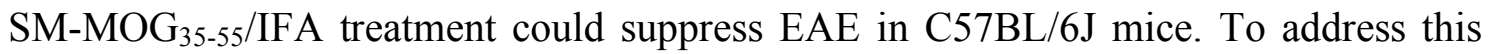
question, I treated C57BL/6J mice with $1 \mu \mathrm{g}$ of $\mathrm{SM}-\mathrm{MOG}_{35-55} / \mathrm{IFA}$ s.c. five days after EAE induction. Age- and sex-matched C57BL/6J mice left untreated were used as the experimental controls. As shown in Figure 3.6, the $\mathrm{SM}-\mathrm{MOG}_{35-55} / \mathrm{IFA}$ treatment did not significantly suppress EAE in C57BL/6J mice. This lack of EAE suppression by 
SM-MOG $_{35-55} / \mathrm{IFA}$ was not simply due to the dose, because doses up to $10 \mu \mathrm{g}$ of

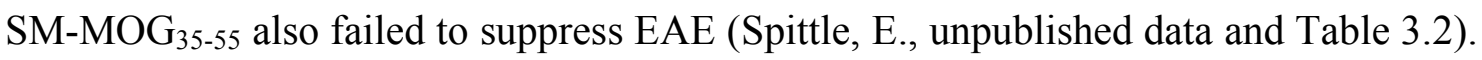
Therefore, the SM-MOG $35-55$ /IFA treatment failed to suppress EAE in contrast to the results reported previously ${ }^{168}$. This was due to the failure of the conjugate treatment and not due to differences between mouse strains.
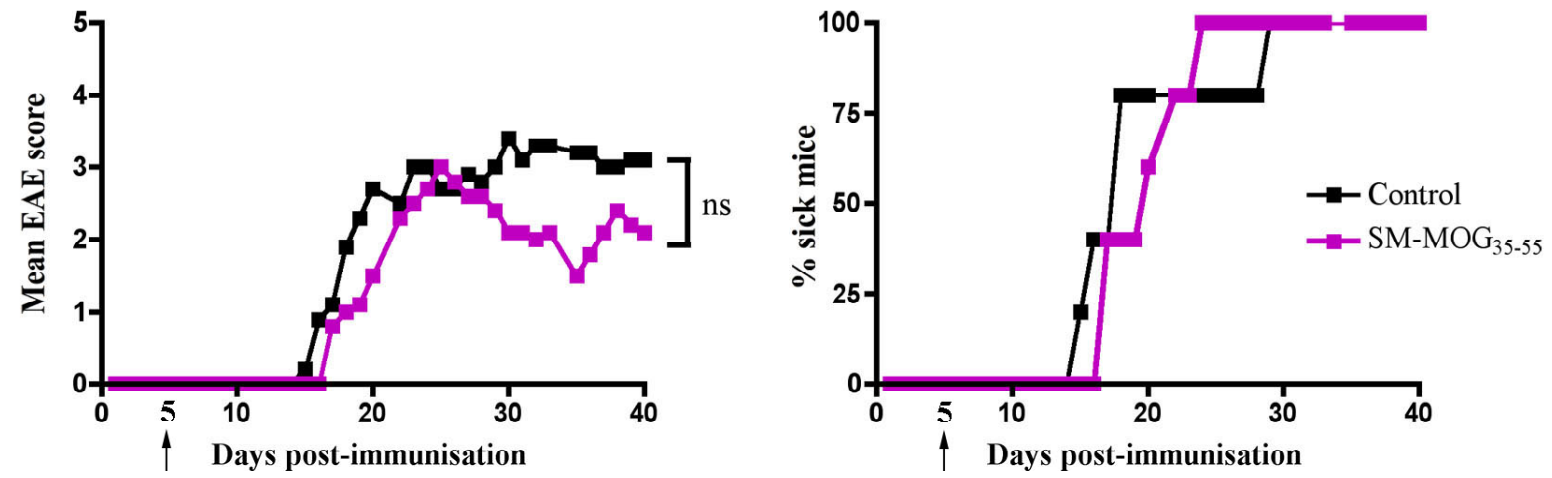

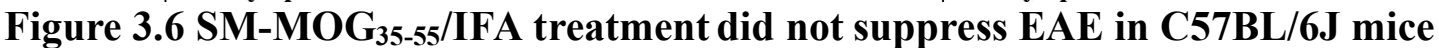
Five days after EAE induction, the $\mathrm{C} 57 \mathrm{BL} / 6 \mathrm{~J}$ mice were treated with either $1 \mu \mathrm{g}$ of SM-MOG $35-55$ in IFA s.c. or left untreated as the experimental controls. Each group contained five mice. Left graph, mean clinical scores. Right graph, \% sick mice. Significant differences are indicated, ns, not significant $(p>0.05)$. Analysis of statistical significance was performed using a one tailed Mann Whitney U test. Results are representative of more than three separate experiments. Arrows indicate the day of

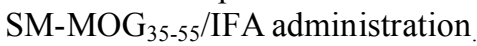

\subsubsection{Adoptive transfer of $\mathrm{CD}^{+}$cells or crude DLN cells from the}

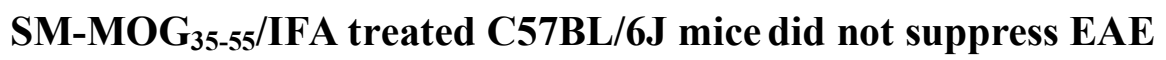

To understand why the $\mathrm{SM}-\mathrm{MOG}_{35-55} / \mathrm{IFA}$ treatment did not significantly suppress EAE, I decided to investigate whether adoptive transfer of the $\mathrm{CD} 25^{+}$cells from SM-MOG $35-55 /$ IFA treated mice could suppress EAE. I purified CD25 $5^{+}$cells from the DLNs of SM-MOG ${ }_{35-55} /$ IFA treated mice and adoptively transferred these cells into recipient mice $\left(2 \times 10^{5}\right.$ cells per mouse). Age- and sex-matched C57BL/6J mice without cell transfer were used as the experimental controls. Two days after adoptive transfer of $\mathrm{CD} 25^{+} \mathrm{T}$ cells, the control and recipient mice were induced with EAE. As shown in Figure $3.7 \mathrm{~A}$, the adoptive transfer of $\mathrm{CD} 25^{+}$cells from the SM-MOG $35-55 / \mathrm{IFA}$ treated mice did not protect the recipient mice from EAE. On day 15, all the recipient mice of $\mathrm{CD} 25^{+}$cells developed severe EAE and the experiment was terminated. Adoptive transfer of up to $2 \times 10^{7}$ crude DLN cells from the SM-MOG ${ }_{35-55} /$ IFA treated mice also failed to suppress EAE (Figure 3.7B). Together, the results from these EAE experiments suggest that the $\mathrm{SM}-\mathrm{MOG}_{35-55} / \mathrm{IFA}$ treatment could not suppress EAE via Tregs as reported previously ${ }^{168}$. Further studies need to be done to re-establish the 
SM-MOG $35-55$ mediated in vivo suppression of EAE before the protective cells that mediated this suppression can be identified.

A
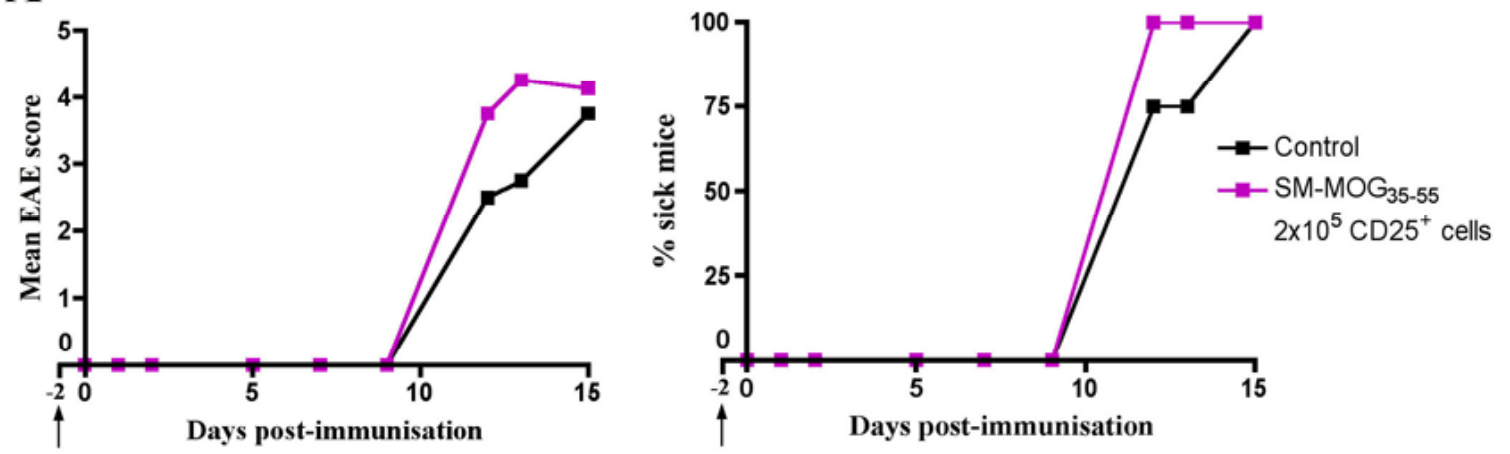

B
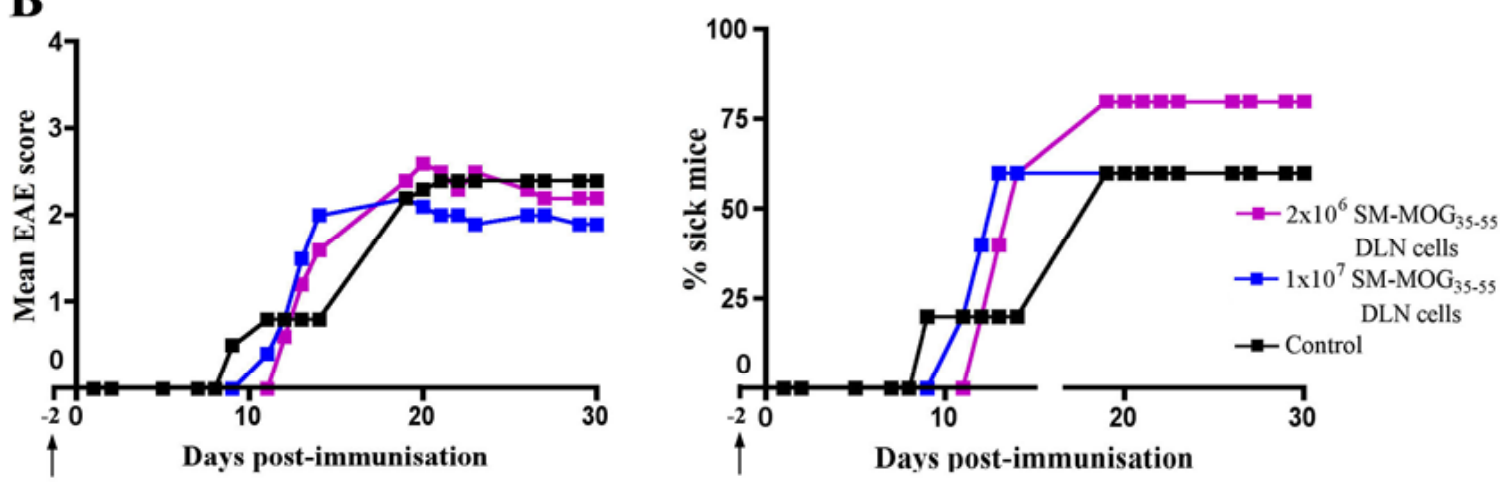

Figure 3.7 Adoptive transfer of neither $\mathrm{CD}^{+}$cells nor crude DLN cells from SM-MOG $35-55 /$ IFA treated mice conferred EAE suppression

C57BL/6J mice were treated with $1 \mu \mathrm{g}$ of SM-MOG $35-55 / \mathrm{IFA}$ s.c. On day five, $2 \times 10^{5}$ purified $\mathrm{CD}^{2} 5^{+}$cells (A) or crude cells $\left(2 \times 10^{6}\right.$ or $1 \times 10^{7}$ cells per mouse) from the DLNs (B) were adoptively transferred to the recipient mice. On day two, the recipient mice were induced with EAE. Age- and sex-matched mice were i.v. injected with $200 \mu \mathrm{l}$ PBS as the experimental controls. Left graph, mean clinical scores. Right graph, \% sick mice. Each group contained four (A) or five mice (B). Arrows indicate the days of cell adoptive transfer.

\subsubsection{Validation of the SM-MOG $35-55$ treatment for EAE}

Because the effect of the original SM- $\mathrm{MOG}_{35-55} / \mathrm{IFA}$ treatment of EAE could not be repeated, and the $\mathrm{SM}-\mathrm{MOG}_{35-55}$ conjugate used in the previous study was not available in our laboratory any longer, a range of experiments were carried out to try to re-establish the immunosuppressive activity of $\mathrm{SM}-\mathrm{MOG}_{35-55}$. Although $\mathrm{SM}-\mathrm{MOG}_{35-55}$ slightly suppressed EAE when it was mixed into the EAE inducing emulsion and administered to mice on day zero s.c. (Figure 3.8), in general, none of the administration protocols (Table 3.2) could suppress EAE to the same extent as had been observed in the previous study ${ }^{168}$. However, minor suppression was observed in a variety of administration routes, suggesting the suppressive activity by $\mathrm{SM}-\mathrm{MOG}_{35-55}$ in vivo. 
Table 3.2 Variables for the optimisation of the SM-MOG 35-55 treatment for EAE

\begin{tabular}{|c|l|}
\hline Dose of SM-MOG 35-55 & $100 \mathrm{ng}, 200 \mathrm{ng}, 500 \mathrm{ng}, 1 \mu \mathrm{g}, 2 \mu \mathrm{g}, 5 \mu \mathrm{g}$ \\
\hline Adjuvant & $\alpha$-galactosyl ceramide $(\alpha \mathrm{GalCer})$ Pam3Cys \\
\hline $\begin{array}{c}\text { Administration route } \\
\begin{array}{c}\text { Administration time (days } \\
\text { post EAE induction) }\end{array}\end{array}$ & $\begin{array}{l}\text { i.p. in CFA or IFA; s.c. in CFA or IFA; i.v; } \\
\text { epicutaneous injection }\end{array}$ \\
\hline PTxn administration & $\begin{array}{l}\text { Single injection of 250 ng PTxn per mouse at day one } \\
\text { Two injections of } 200 \mathrm{ng} \text { PTxn each time each mouse } \\
\text { at days 0 and two }\end{array}$ \\
\hline Others & $\begin{array}{l}\text { Using SM-MOG } \\
\text { MOG protein induced EAE }\end{array}$ \\
\hline
\end{tabular}
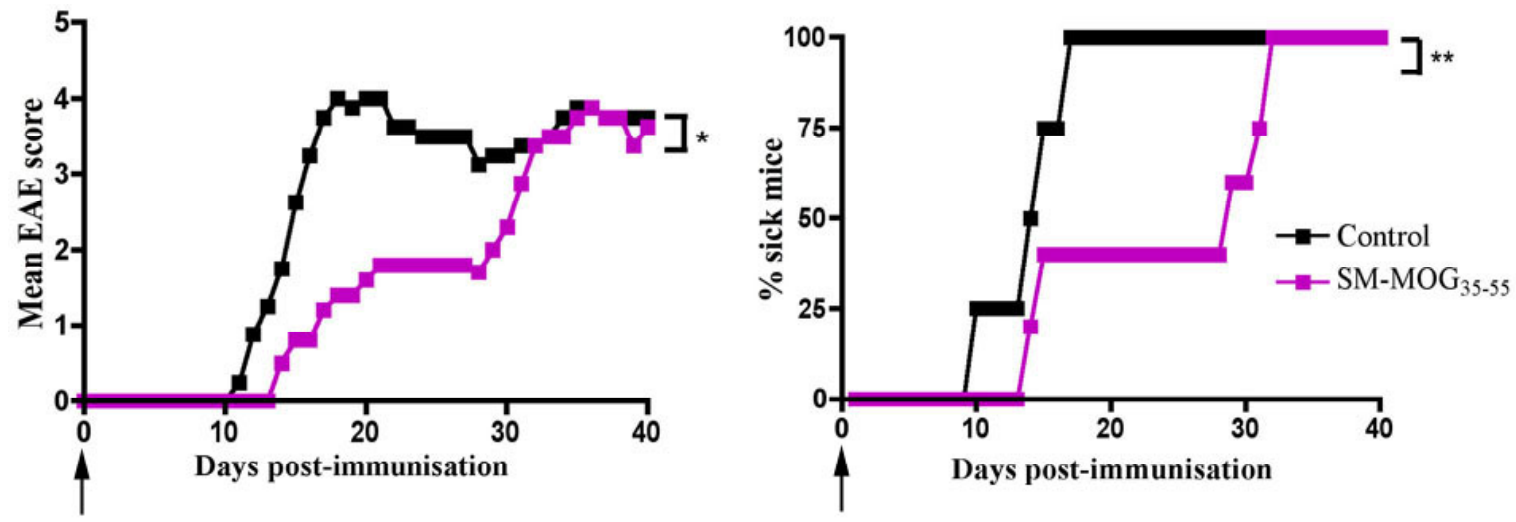

Figure 3.8 The addition of SM-MOG S5-55 $_{35}$ to the EAE inducing emulsion caused a slight suppression of EAE

C57BL/6J mice were immunised with $50 \mu \mathrm{g}$ of $\mathrm{MOG}_{35-55}$ emulsified in CFA plus $2 \mu \mathrm{g}$ of SM-MOG $35-55$. Mice immunised with $50 \mu \mathrm{g}$ of $\mathrm{MOG}_{35-55}$ emulsified in CFA were employed as the experimental controls. On day one, all the mice were treated with $250 \mathrm{ng}$ PTxn i.p. Left graph, mean clinical scores. Right graph, \% sick mice. The control group contained four mice and $\mathrm{SM}-\mathrm{MOG}_{35-55}$ group contained five mice. One of the mice from the $\mathrm{SM}-\mathrm{MOG}_{35-55}$ group was culled on day 30 due to peritonitis. Significant differences are indicated, ${ }^{*} \mathrm{p}<0.05,{ }^{* *} \mathrm{p}<0.01$. Analysis of statistical significance was performed using a one tailed Mann Whitney U test.

\subsubsection{Identification of the SM targeting cells}

Despite the apparent weaker effect of the new $\mathrm{SM}-\mathrm{MOG}_{35-55}$ compared to the previous study ${ }^{168}$, suppression of EAE was still observed. As adoptive transfer of neither CD $25^{+}$ nor Foxp $3^{+}$cells conferred EAE suppression (Section 3.3.2), I hypothesised that other cell types may be responsible for the minor suppression of EAE mediated by the administration of $\mathrm{SM}-\mathrm{MOG}_{35-55}$. In order to identify the cellular source of the

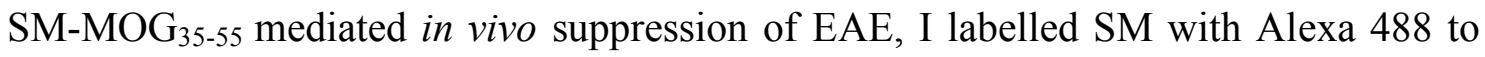
distinguish the SM-associating cells. The labelling of SM is described in detail in Section 2.14.3. 


\subsubsection{SM bound to $\mathrm{MHCII}^{+}$cells in vitro and in vivo}

The staining of SM-Alexa 488 was first examined on the splenocytes from C57BL/6J mice in vitro. As shown in Figure 3.9, when SM-Alexa 488 was incubated with the spleen or lymph node cells (LN cells) at room temperature for 10 minutes, extensive staining of SM-Alexa 488 was observed on $\mathrm{MHCII}^{+}$Thy $1.2^{-}$cells.
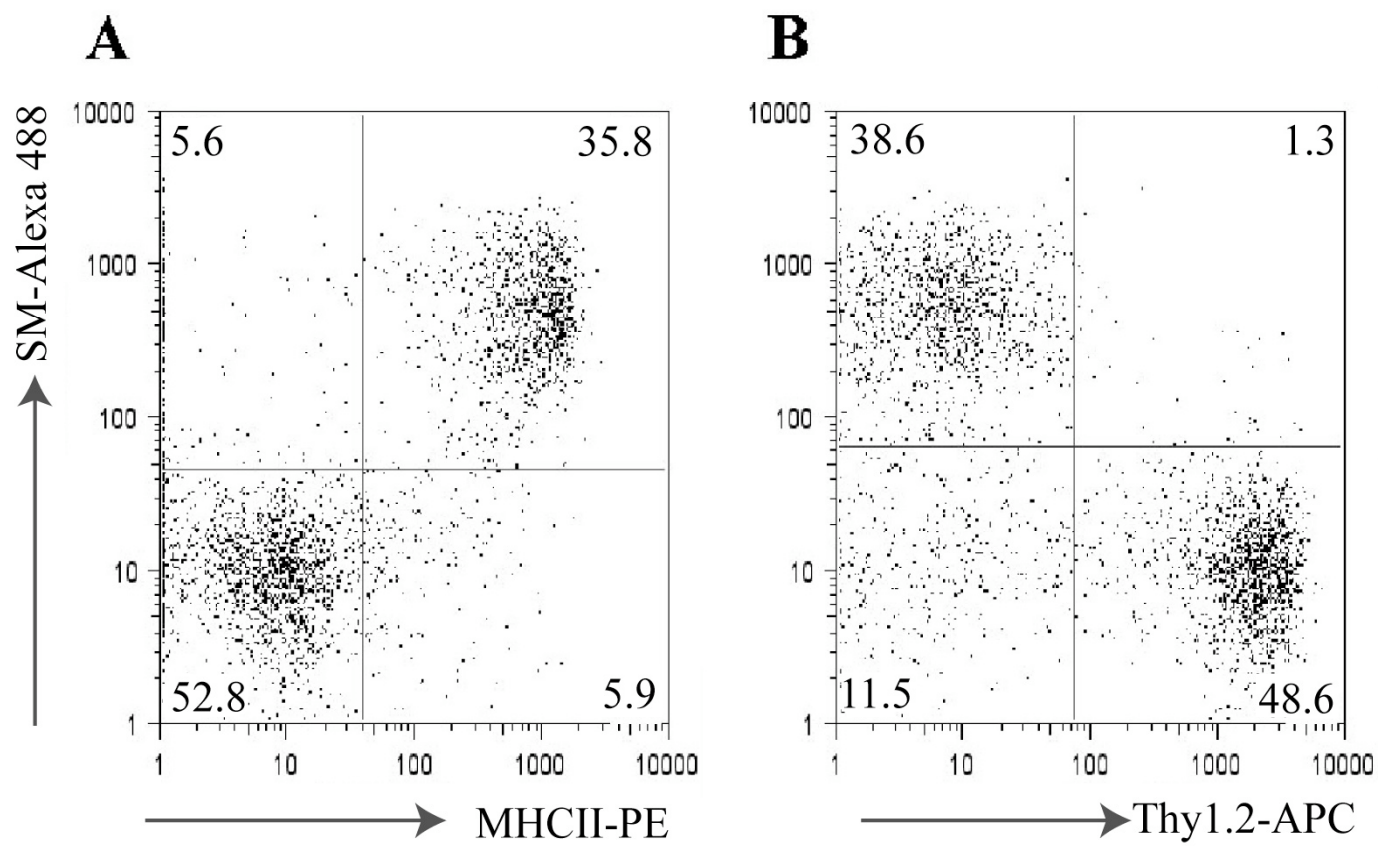

Figure 3.9 SM-Alexa 488 was captured by the $\mathrm{MHCII}^{+}$Thy1.2- ${ }^{-}$splenocytes in vitro The C57BL/6J mouse splenocytes were incubated with SM-Alexa 488 at room temperature for 10 minutes and analysed by flow cytometry. The $\mathrm{SM}^{+}$cells were (A) $\mathrm{MHCII}^{+}$, and (B) Thy $1.2^{-}$. Numbers indicate percent of total cells in each quadrant. Results are representative of more than two separate experiments.

To confirm that the same population was targeted by $\mathrm{SM}_{-} \mathrm{MOG}_{35-55}$ in vivo, SM-Alexa 488 was injected into mice i.v. to positively identify the SM-associating cells. Age- and sex-matched mice injected with the same molar dose of OVA-Alexa 488 or PBS were used as the experimental controls. Extensive Alexa 488 fluorescence was observed on $\mathrm{MHCII}^{+}$Thy $1.2^{-}$cells in the spleens (Figure 3.10), lymph nodes (data not shown) and blood (data not shown) of the C57BL/6J mice injected with SM-Alexa 488. The Alexa 488 fluorescence was significantly reduced in the MHCII deficient (MHCII-/-) mice injected with SM-Alexa 488, indicating the MHCII-dependent uptake of SM. Minimal staining was observed on $\mathrm{MHCII}^{+}$cells when OVA-Alexa 488 or PBS was used. 

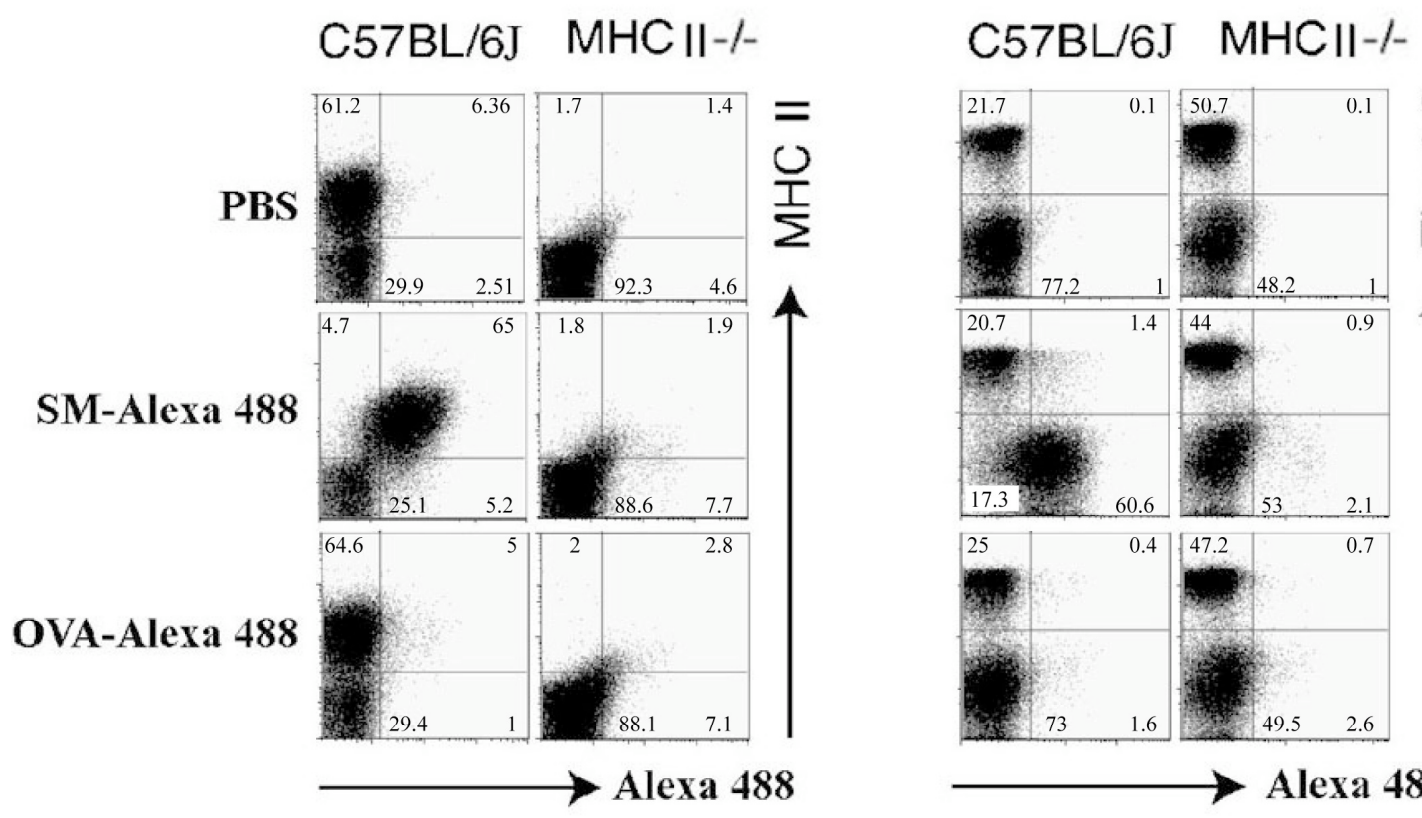

Figure 3.10 SM was captured by $\mathrm{MHCII}^{+}$cells in vivo

SM or OVA proteins were labelled with Alexa 488 dye and injected into either C57BL/6J or MHCII-/mice i.v. (50 $\mu \mathrm{g}$ of SM-Alexa 488 per mouse and $75 \mu \mathrm{g}$ of OVA-Alexa 488). Three hours after the injections, splenocytes from the injected mice were collected and analysed by flow cytometry for the indicated proteins. Numbers indicate percent of total cells in each quadrant. Results are representative of more than three separate experiments.

As shown in Figure 3.11, the MHCII ${ }^{+}$cells targeted by SM-Alexa 488 included B cells $\left(\mathrm{B} 220^{+} \mathrm{CD} 11 \mathrm{c}^{-}\right)$, macrophages $\left(\mathrm{F} 4 / 80^{+} \mathrm{CD} 11 \mathrm{c}^{-}\right)$, DCs $\left(\mathrm{CD} 11 \mathrm{c}^{+}\right)$and Langerin $^{+}$cells (data not shown), indicating SM does not preferentially bind to a particular APC population. Significant SM-Alexa 488 staining was observed on all major splenic DC subsets $\left(\mathrm{CD}^{+} \mathrm{CD} 8^{-} \mathrm{DC}, \mathrm{CD} 4^{-} \mathrm{CD} 8^{+} \mathrm{DC}\right.$ and $\left.\mathrm{CD} 4^{-} \mathrm{CD} 8^{-} \mathrm{DC}\right)$. Expression of $\mathrm{CD} 40, \mathrm{CD} 80$ and CD86 was unchanged on the DCs targeted by SM-Alexa 488 (Figure 3.12), suggesting the interaction of SM with MHCII does not induce the maturation of $\mathrm{SM}^{+}$ DCs during this period. Interestingly, injection of SM-Alexa 488 also resulted in weak fluorescence on an $\mathrm{MHCII}^{-}$cell population in the blood (will be discussed in the next section). This fluorescence was not observed in mice injected with PBS and only marginal fluorescence was observed in mice injected with OVA-Alexa 488. These results reveal an MHC-independent uptake mechanism of SM by these cells. 


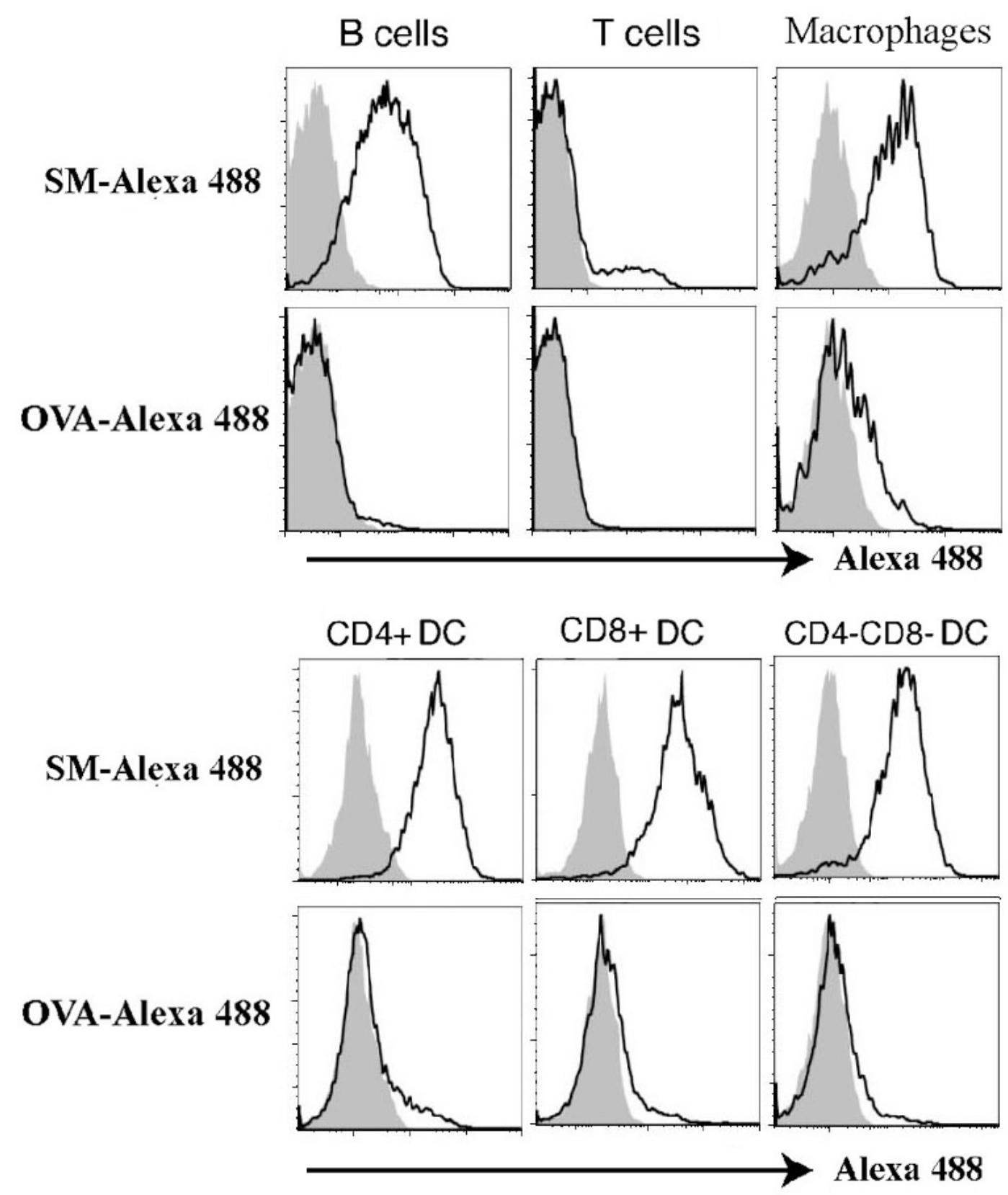

Figure 3.11 SM targeted all the major $\mathrm{MHCII}^{+}$cells in the spleen

SM-Alexa 488 or OVA-Alexa 488 was injected into C57BL/6J mice i.v. $(50 \mu \mathrm{g}$ of SM-Alexa 488 or $75 \mu \mathrm{g}$ of OVA-Alexa 488 per mouse). Three hours after the injections, the splenocytes from injected mice were collected and analysed by flow cytometry. Cellular fluorescence was assessed on splenic B cells $\left(\mathrm{B} 220^{+}\right)$, $\mathrm{T}$ cells $\left(\right.$ Thy $\left.1.2^{+}\right)$, macrophages $\left(\mathrm{F} 4 / 80^{+}\right)$, and the major splenic DC subtypes $\left(\mathrm{CD} 11 \mathrm{c}^{+} \mathrm{CD} 4^{+}\right.$, $\left.\mathrm{CD} 11 \mathrm{c}^{+} \mathrm{CD} 8^{+} \mathrm{CD} 11 \mathrm{c}^{+} \mathrm{CD} 4^{-} \mathrm{CD} 8^{-}\right)$. Grey profiles are from mice injected with PBS only. Results are representative of more than two separate experiments. 


\section{Dendritic cells}

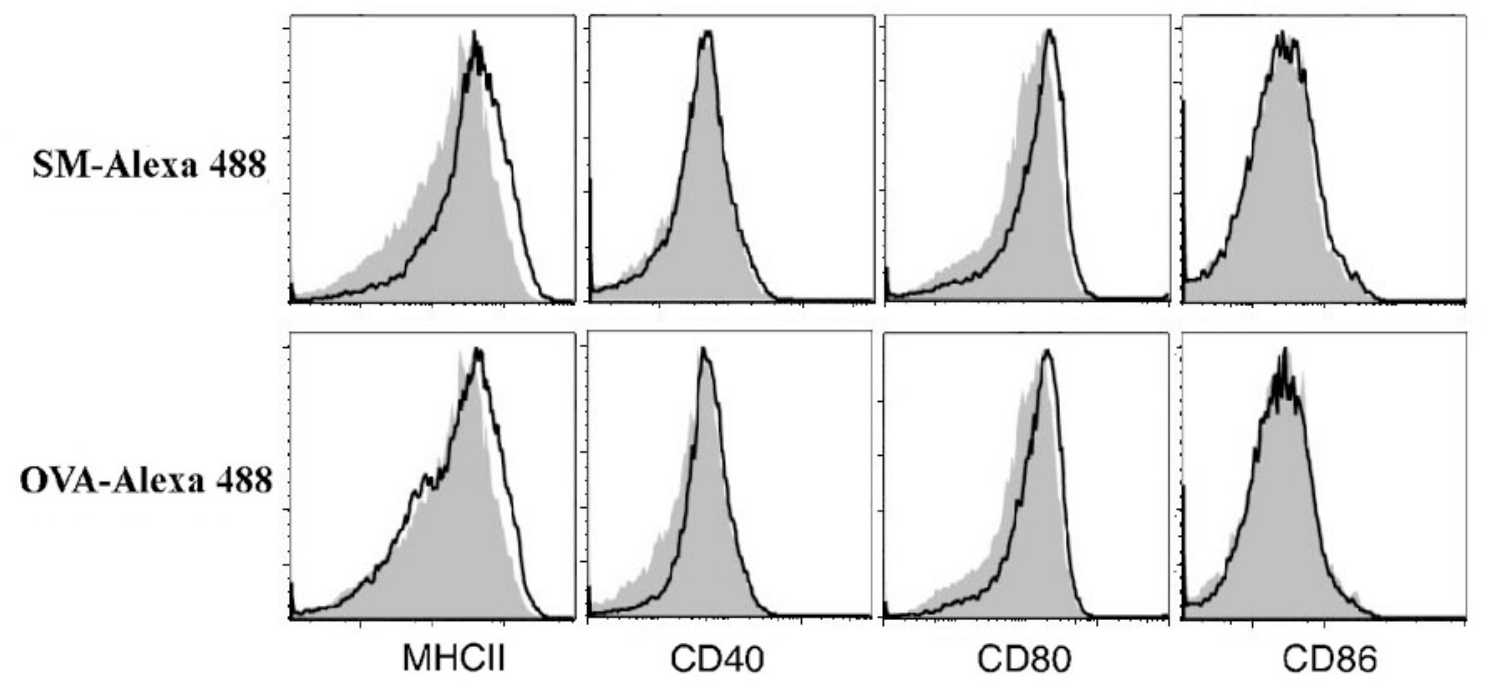

Figure 3.12 SM binding to DCs did not change the cells' maturation markers

SM-Alexa 488 or OVA-Alexa 488 was injected into C57BL/6J mice i.v. $(50 \mu \mathrm{g}$ of SM-Alexa 488 or $75 \mu \mathrm{g}$ of OVA-Alexa 488 per mouse). Three hours after the injections, the splenocytes from injected mice were collected and analysed by flow cytometry. Expression of maturation markers CD40, CD80 and CD86, and MHCII was assessed on gated splenic DC (CD11 ${ }^{+}$cells) from mice treated with the fluorescent molecules. Grey profiles are from mice injected with PBS only. Results are representative of more than two separate experiments.

\subsubsection{SM was captured by an $\mathrm{MHCII}^{-}$cell population in the blood}

To further confirm whether an MHCII-independent mechanism existed for uptake of SM, I looked at the uptake of SM-Alexa 488 in MHCII-/- mice compared to the wild type mice. As shown in Figure 3.13, a distinct $\mathrm{MHCII}^{-}$cell population in the blood of both MHCII-/- and wild type mice i.v. injected with SM-Alexa 488 showed positive staining of SM-Alexa 488. The staining of SM-Alexa 488 in MHCII-/- cells indicates an MHC-independent uptake mechanism for SM. The marginal staining observed in the mice injected with OVA-Alexa 488 excludes the possibility of SM uptake via antigen-non-specific phagocytosis. Weak SM-Alexa 488 staining was also observed on $\mathrm{MHCII}^{-}$cells in the spleens (Figure 3.10) but not in the lymph nodes (data not shown).

The $\mathrm{SM}^{+} \mathrm{MHCII}$ cells in the blood demonstrated a high level of CD11b and intermediate levels of F4/80 and Gr-1 staining (Figure 3.14) and did not express any $\mathrm{T}$ cell, B cell or DC markers including Thy1.2, B220 and CD11c (data not shown). The binding of SM to this blood $\mathrm{MHCII}^{-}$cell population raised the possibility that these $\mathrm{SM}^{+} \mathrm{MHCII}^{-}$cells may play a role in the $\mathrm{SM}-\mathrm{MOG}_{35-55}$ mediated suppression of EAE. Further investigations around the phenotype and function of this $\mathrm{SM}^{+} \mathrm{MHCII}$ blood population are presented in Chapter 4. 


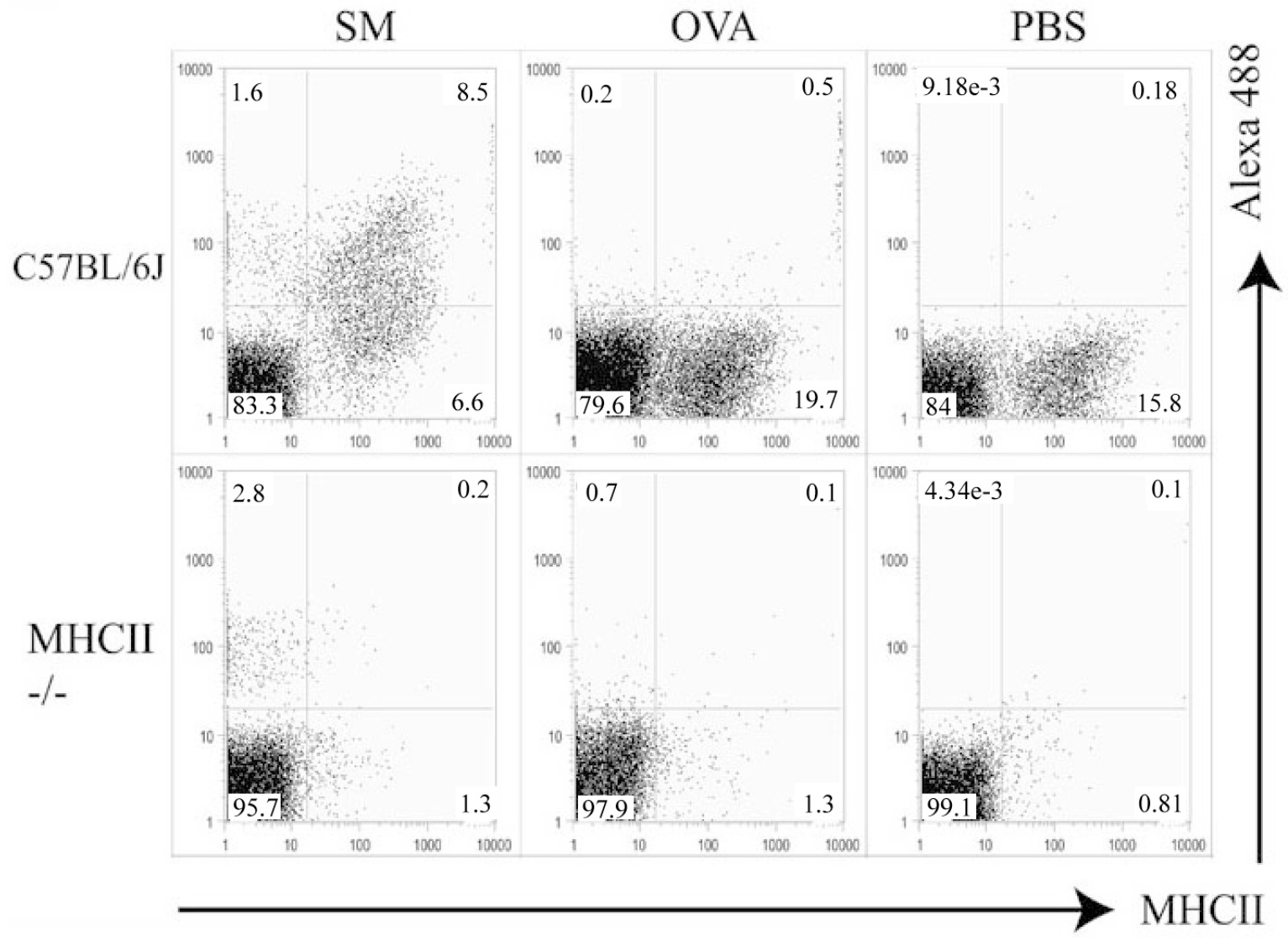

Figure 3.13 A blood-borne MHCII cell population captured SM-Alexa 488

SM-Alexa 488, OVA-Alexa 488 or PBS were injected into either C57BL/6J or MHCII-/- mice i.v. (50 $\mu \mathrm{g}$ of SM-Alexa 488 or $75 \mu \mathrm{g}$ of OVA-Alexa 488 per mouse). Three hours after the injections, blood cells from the injected mice were collected and analysed by flow cytometry for the staining of the indicated proteins. Numbers indicate percent of total cells in each quadrant. Results are representative of more than three separate experiments. 

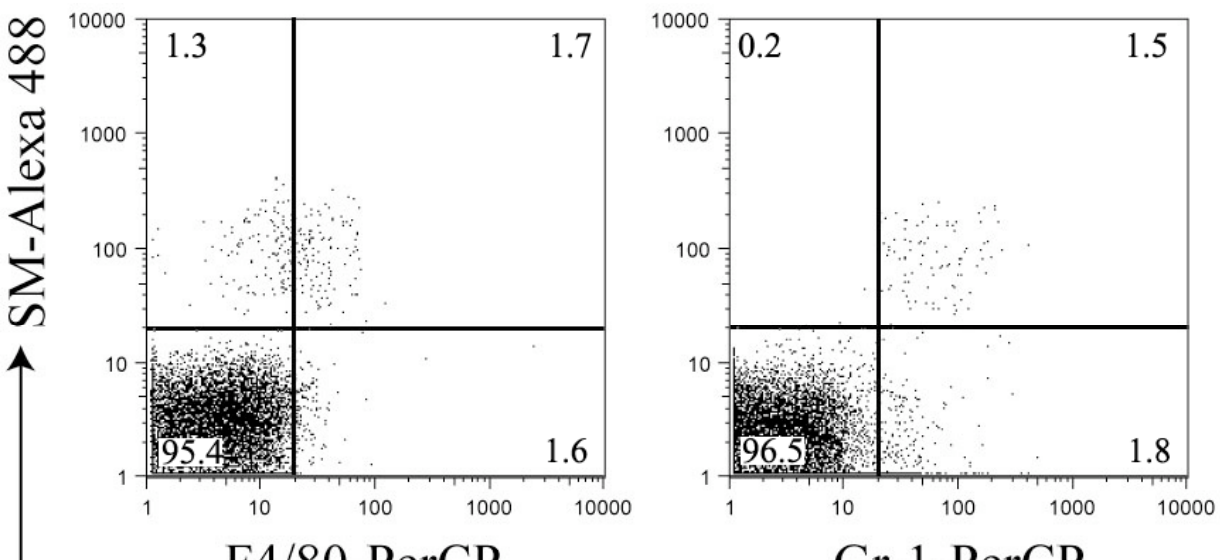

F4/80-PerCP

Gr-1-PerCP

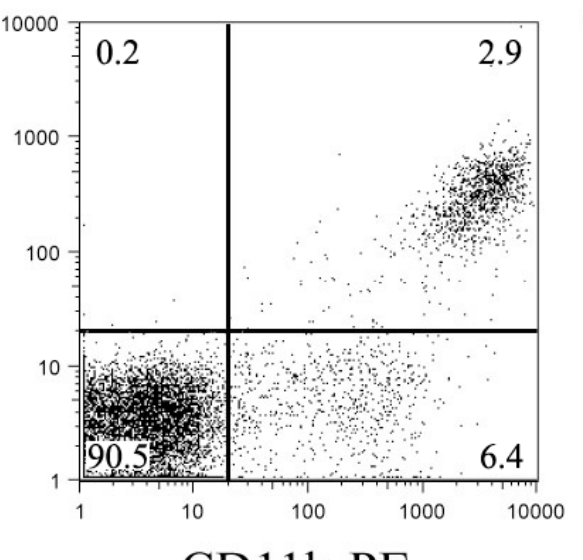

CD11b-PE

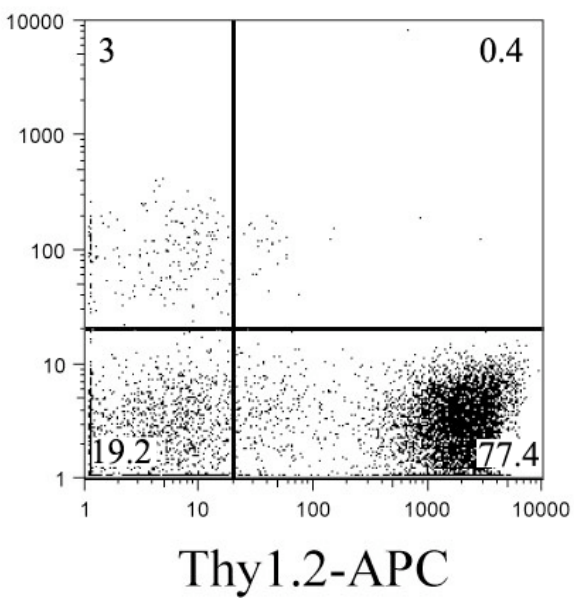

Figure 3.14 $\mathrm{SM}^{+} \mathrm{MHCII}^{-}$cells in the blood express $\mathrm{F4} / 80, \mathrm{Gr}-1$ and $\mathrm{CD} 11 \mathrm{~b}$

SM-Alexa 488 was injected into MHCII-/- mice i.v. (50 $\mu \mathrm{g}$ of SM-Alexa 488 per mouse). Three hours after the injections, blood cells from the injected mice were collected and analysed by flow cytometry for the staining of SM-Alexa 488. Numbers indicate percent of total cells in each quadrant. Data shown are representative from more than three separate experiments.

\subsection{Discussion}

The aim of the experiments presented in this chapter was to understand the cellular and molecular changes involved in the SM-MOG $\mathrm{MO}_{35-55} / \mathrm{IFA}$ mediated immune suppression of EAE, with the focus on the role of $\mathrm{MOG}_{35-55}$ specific Tregs in this process. However, treating mice with $\mathrm{SM}-\mathrm{MOG}_{35-55} / \mathrm{IFA}$ or adoptive transfer of either the Foxp3 ${ }^{+}$Tregs or $\mathrm{CD} 25^{+}$Tregs from SM-MOG $35-55 /$ IFA treated mice did not suppress EAE as expected.

In a previous $\operatorname{study}^{168}, \mathrm{SM}^{\mathrm{M}} \mathrm{MOG}_{35-55}$ was shown to be $\sim 100$ times more potent than $\mathrm{MOG}_{35-55}$ alone at activating 2D2 $\mathrm{T}$ cell response in vitro. However, SM-MOG $35-55$ failed to induce EAE in the $\mathrm{MOG}_{35-55}$ specific TCR transgenic 2D2 mice, leading to the hypothesis that $\mathrm{SM}-\mathrm{MOG}_{35-55}$ might activate suppressive mechanisms in vivo (e.g. $\mathrm{MOG}_{35-55}$ specific Tregs) and eventually the finding that SM-MOG $35-55 / \mathrm{IFA}$ 
administration markedly suppressed EAE. In this study, SM-MOG $35-55$ showed similar characteristics as described in the previous study, including the high-potency activation of 2D2 T cell response in vitro (Figure 3.2) and the failure to induce EAE in 2D2 mice (Spittle E., unpublished data). However, the $\mathrm{SM}_{-} \mathrm{MOG}_{35-55} / \mathrm{IFA}$ administration to mice did not significantly suppress EAE in C57BL/6J mice as previously reported.

Although a large number of experiments were carried out in our laboratory, the reason for the failure of EAE suppression by $\mathrm{SM}-\mathrm{MOG}_{35-55} / \mathrm{IFA}$ remains elusive. The failure of the $\mathrm{SM}_{-} \mathrm{MOG}_{35-55} / \mathrm{IFA}$ treatment to suppress EAE was not due to the dose or timing of administration (Table 3.2). Other possibilities for the lack of suppression include the unknown differences between the SM in this study and the one made for the previous study (from the University of Auckland), or difference in disease induction between the animal facilities where the initial study was done (at the University of Otago, Wellington School of Medicine and Health Sciences) compared to the institute's new facility (at Malaghan Institute of Medical Research).

In various experimental conditions for the optimisation of treating EAE using

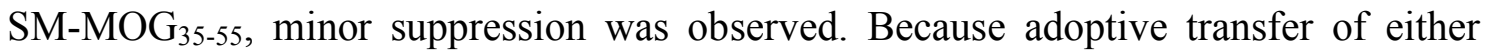
$\mathrm{CD}_{25} 5^{+}$or $\mathrm{Foxp}^{+}$Tregs from the $\mathrm{SM}-\mathrm{MOG}_{35-55}$ treated mice did not confer protection to recipient mice, I hypothesised other cell types were responsible for this minor suppression of EAE mediated by $\mathrm{SM}-\mathrm{MOG}_{35-55}$. This hypothesis was also supported by the experimental data from the previous study showing that depleting of Tregs prior to

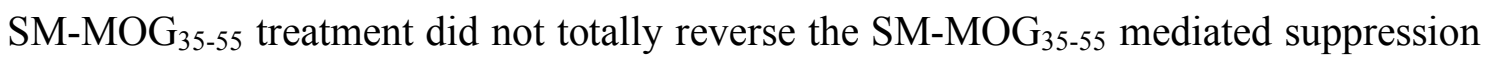
of $\mathrm{EAE}^{168}$.

In order to identify what cells might mediate the suppression of EAE by SM-MOG $35-55$, I injected SM-Alexa 488 into mice to fluorescently label the cells capturing SM. As shown in Figure 3.11, after i.v. injection, SM was captured by all the major APCs and the binding of SM to these APCs did not change the cells' maturation status during this period (Figure 3.12). Interestingly, an $\mathrm{MHCII}^{-}$cell population in the blood showed the staining of SM. The blood cells from mice injected with OVA-Alexa 488 only showed marginal fluorescence, indicating the uptake of SM was not due to antigen-non-specific phagocytosis. The uptake of superantigen by these blood $\mathrm{MHCII}^{-}$cells has not been reported before. The SM staining was also observed in the MHCII-/- mice injected i.v. 
with SM-Alexa 488, indicating an MHCII independent uptake mechanism by SM. The uptake of SM by these blood $\mathrm{MHCII}^{-}$cells was investigated further in Chapter 4.

The $\mathrm{SM}^{+} \mathrm{MHCII}^{-}$cells were shown to express F4/80, Gr-1 and CD11b in this chapter. In the literature, these cell surface antigens have been reported to be expressed by tumour myeloid-derived suppressor cells (MDSCs) that are potent suppressors of immune responses $^{45,75,76}$. However, MDSCs have not been identified in naïve mouse blood. It is possible that these blood $\mathrm{SM}^{+} \mathrm{MHCII}^{-}$cells are suppressor cells of the immune responses, i.e. a subpopulation of MDSCs that has not been described before and that the binding of $\mathrm{SM}_{-} \mathrm{MOG}_{35-55}$ to these cells enhanced their suppressive activity in vivo. This possibility was investigated in Chapter 4.

\subsection{Conclusions}

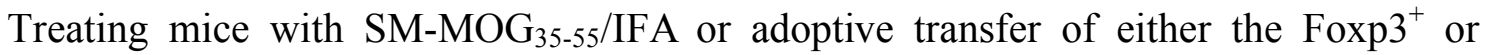
$\mathrm{CD}_{25^{+}}$Tregs from SM-MOG $35-55 /$ IFA treated mice did not significantly suppress EAE. However, the administration of $\mathrm{SM}-\mathrm{MOG}_{35-55}$ to mice using various methods repeatedly showed minor suppressive effect of EAE, suggesting the in vivo suppressive feature of SM-MOG $35-55$. After being injected into mice i.v., SM was captured by a blood $\mathrm{MHCII}^{-}$ $\mathrm{CD} 11 \mathrm{~b}^{+} \mathrm{F} 4 / 80^{+} \mathrm{Gr}-1^{+}$cell population. Cells expressing the same phenotype have been reported to be suppressor cells MDSCs in mice with tumours. This raised the possibility that the $\mathrm{SM}^{+} \mathrm{MHCII} C \mathrm{CD} 11 \mathrm{~b}^{+} \mathrm{F} 4 / 80^{+} \mathrm{Gr}-1^{+}$cells are suppressor cells and are important in the minor suppression of EAE observed in the $\mathrm{SM}-\mathrm{MOG}_{35-55}$ treated mice. This possibility was investigated in Chapter 4 and Chapter 5 . 


\section{Chapter 4:}

\section{DM Binds to Blood Myeloid-Derived Suppressor Cells}




\subsection{Introduction}

Myeloid-derived suppressor cells (MDSCs) are a population of $\mathrm{Gr}-1^{+} \mathrm{CD} 11 \mathrm{~b}^{+}$ suppressive myeloid cells that have been described in different diseases and in particular, have been extensively studied in tumours ${ }^{45,71-76}$. As suggested by their name, MDSCs are potent suppressors of immune responses and are considered critical in assisting tumours to escape immune recognition ${ }^{45,75,76}$. MDSCs are a heterogenous population of myeloid cells, which include immature macrophages, monocytes, neutrophils and $\mathrm{DCs}^{45,76}$ and have been identified in most patients and mice bearing tumours ${ }^{76}$.

Various mechanisms have been reported to be utilised by MDSCs to suppress $\mathrm{T}$ cell responses. For example, in mice, MDSCs have been shown to suppress T cell activation via up-regulation of NO production ${ }^{78,79}$ and/or arginase 1 induction ${ }^{80-83,194}$. NO inhibits $\mathrm{T}$ cell activation via reversible disruption of the Jak/STAT5 signalling pathway ${ }^{79}$; whereas arginase 1 suppresses T cell activation by depleting extracellular L-arginine, a conditionally essential amino acid that is critical for $\mathrm{T}$ cell function ${ }^{80-83}$. It has also been reported that the suppressive activity of MDSCs is mediated by Tregs ${ }^{74}$. The reason for the diverse suppressive mechanisms of MDSCs is likely due to the suppression performed by different cells within the heterogenous MDSC population, e.g. mononuclear MDSCs vs. granulocytic MDSCs.

The lack of precision in identifying the suppressive population(s) within the heterogenous MDSCs has been problematic. In mice, MDSCs are defined by the co-expression of CD11b and Gr-1, however, both mononuclear cells and granulocytes express these two markers. The mononuclear MDSCs and granulocytic MDSCs are functionally different and use distinct mechanisms to suppress immune responses. For example, in the EG7 lymphoma model, mononuclear MDSCs preferentially suppress T cell responses via up-regulation of NO production, whereas granulocytic MDSCs utilise arginase $1^{71}$. The identification and characterisation of discrete MDSC populations is therefore key in improving our understanding of MDSC function and targeting their use for immunotherapy.

In the previous chapter, I identified a small population of $\mathrm{MHCII}^{-}$cells in blood that stained positive for SM following i.v. injection of SM-Alexa 488. These $\mathrm{SM}^{+}$blood 
cells express CD11b, Gr-1 and F4/80, the markers that have previously been reported to be expressed by the MDSCs in tumours ${ }^{45,75,76}$. I therefore hypothesised that these $\mathrm{MHCII}^{-} \mathrm{SM}^{+}$blood cells were a population of MDSCs residing in the blood and that these cells played a role in $\mathrm{SM}_{-\mathrm{MOG}_{35-55}}$ mediated suppression of EAE.

However, SM predominantly bound to $\mathrm{MHCII}^{+}$APCs and only a small proportion of $\mathrm{SM}$ bound to the blood $\mathrm{MHCII} \mathrm{CD}^{-} 1 \mathrm{~b}^{+} \mathrm{F} 4 / 80^{+} \mathrm{Gr}-1^{+}$cells, the possible suppressor cells. To improve targeting SM to the blood $\mathrm{MHCII}^{-} \mathrm{CD} 11 \mathrm{~b}^{+} \mathrm{F} 4 / 80^{+} \mathrm{Gr}-1^{+}$cells, it would therefore be necessary to prevent SM-MHCII binding. Professor John Fraser from Auckland University kindly provided another mSAg, double mutant SMEZ-2 (DM), for this purpose. DM is a mutant toxoid of the wild-type SMEZ-2 isolated from S. pyogenes strain 2035, the same strain from which SM is derived. In contrast to SM, DM is defective at both the MHCII and TCR binding sites. In the absence of MHCII binding capability, it was hypothesised that DM would possess an enhanced binding capability to these blood $\mathrm{MHCII}^{-} \mathrm{CD} 11 \mathrm{~b}^{+} \mathrm{F} 4 / 80^{+} \mathrm{Gr}-1^{+}$cells. This feature would allow the identification and potential targeted manipulation of these proposed suppressor cells.

\subsection{Aims}

The overall aim of this chapter was to determine whether the $\mathrm{MHCII}^{-}$ $\mathrm{CD} 11 \mathrm{~b}^{+} \mathrm{F} 4 / 80^{+} \mathrm{Gr}-1^{+}$blood cells were functional suppressor cells and to investigate the potential of the mSAg (SM and DM), to be used for targeted manipulation of these cells.

The specific aims were as follows:

- To confirm whether the MHCII binding capacity of DM was abrogated

- To investigate whether DM was able to bind to the $\mathrm{MHCII}^{-} \mathrm{CD} 11 \mathrm{~b}^{+} \mathrm{F} 4 / 80^{+} \mathrm{Gr}-1^{+}$ blood cells in vivo

- To characterise the phenotype of the $\mathrm{MHCII}{ }^{-} \mathrm{CD} 11 \mathrm{~b}^{+} \mathrm{F} 4 / 80^{+} \mathrm{Gr}-1^{+}$blood cells

- To determine whether the $\mathrm{MHCII}^{-} \mathrm{CD} 11 \mathrm{~b}^{+} \mathrm{F} 4 / 80^{+} \mathrm{Gr}-1^{+}$blood cells were able to suppress $\mathrm{T}$ cell proliferation

- To investigate the mechanisms involved in the $\mathrm{MHCII}^{-} \mathrm{CD} 11 \mathrm{~b}^{+} \mathrm{F} 4 / 80^{+} \mathrm{Gr}-1^{+}$ blood cell-dependent immune suppression 
- To test the potential of mSAg DM for targeted manipulation of the $\mathrm{MHCII}^{-}$ $\mathrm{CD} 11 \mathrm{~b}^{+} \mathrm{F} 4 / 80^{+} \mathrm{Gr}-1^{+}$blood cells.

\subsection{Results}

\subsubsection{The MHCII binding capacity of DM is abrogated}

To confirm that DM is defective in its MHCII binding, two methods were used to examine the DM binding capacity to MHCII. First, DM was labelled with Alexa 488 dye and the DM-Alexa 488 staining was examined on $\mathrm{MHCII}^{+}$cells by flow cytometry. Second, peptides known to bind to MHCII (IA ${ }^{\mathrm{b}}$ ), $\mathrm{MOG}_{35-55}$ and $\mathrm{OVA}_{323-339}$, were conjugated to $\mathrm{DM}$ and the efficiency of the peptide presentation to $\mathrm{CD} 4^{+} \mathrm{T}$ cells was measured.

\subsubsection{1 $\mathrm{MHCII}^{+}$cells do not stain with $\mathrm{DM}$ in vitro or in vivo}

To verify whether the MHCII binding capacity of DM was abrogated, the staining of DM-Alexa 488 was examined on $\mathrm{MHCII}^{+}$cells both in vitro and in vivo. Wild type SMEZ-M2 (WT)-Alexa 488, SM-Alexa 488, OVA-Alexa 488 and PBS were used as experimental controls.

The Alexa 488 labelled proteins were incubated with C57BL/6J splenocytes in vitro at room temperature for 30 minutes. As shown in Figure 4.1A, the staining of DM and the control protein OVA was not observed on $\mathrm{MHCII}^{+}$cells. In contrast, $\mathrm{MHCII}^{+}$cells showed the staining of both WT and SM. The negative staining of DM on MHCII ${ }^{+}$cells indicated that the MHCII binding capacity of DM was abrogated.

Consistent with in vitro data, $\mathrm{MHCII}^{+}$cells only showed minor staining of $\mathrm{DM}$ in vivo. The Alexa 488 labelled proteins were injected into either C57BL/6J or MHCII-/- mice i.v. Three hours after the injection, splenocytes from these mice were collected and analysed by flow cytometry. As shown in Figure 4.1B, in the C57BL/6J mice injected with Alexa 488 labelled DM or OVA, the $\mathrm{MHCII}^{+}$cells only showed marginal fluorescence. In contrast, the $\mathrm{MHCII}^{+}$splenocytes showed extensive staining of SM. WT-Alexa 488 was not tested in vivo due to ethical consideration of its toxicity. Taken together, DM did not bind to $\mathrm{MHCII}^{+}$cells both in vitro and in vivo. 
Interestingly, the fluorescence detected on splenocytes from the C57BL/6J mouse injected with DM-Alexa 488 was greater than that observed after injecting with OVA-Alexa 488 or PBS. This weak DM staining was also observed in the MHCII-/mouse that received DM-Alexa 488, indicating an MHCII-independent uptake mechanism for DM. The staining of DM on the $\mathrm{MHCII}^{-}$cells will be further discussed in Section 4.3.2. 
A

WT

SM

DM
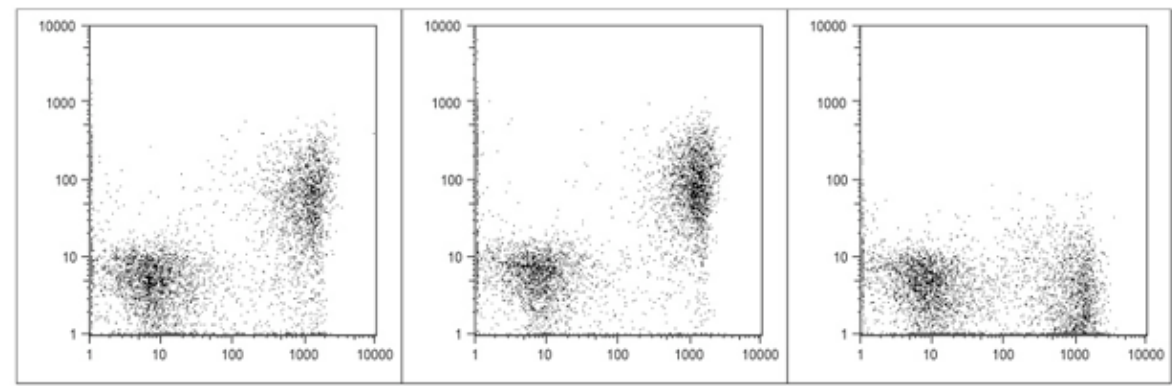

$\underset{\substack{+\underset{+}{\infty}}}{\infty}$

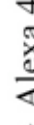

OVA

PBS
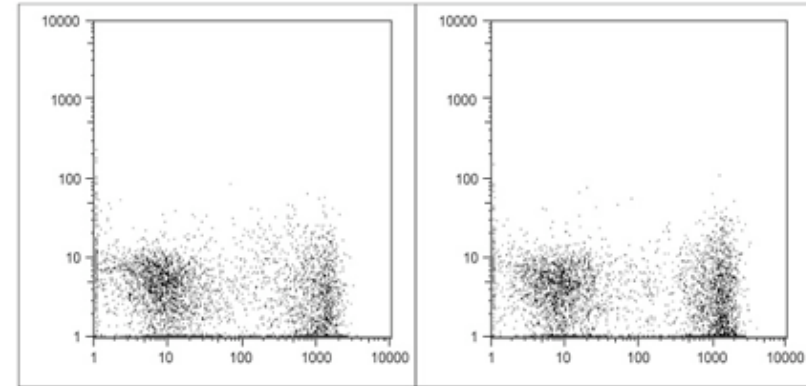

$\mathrm{MHCII}$

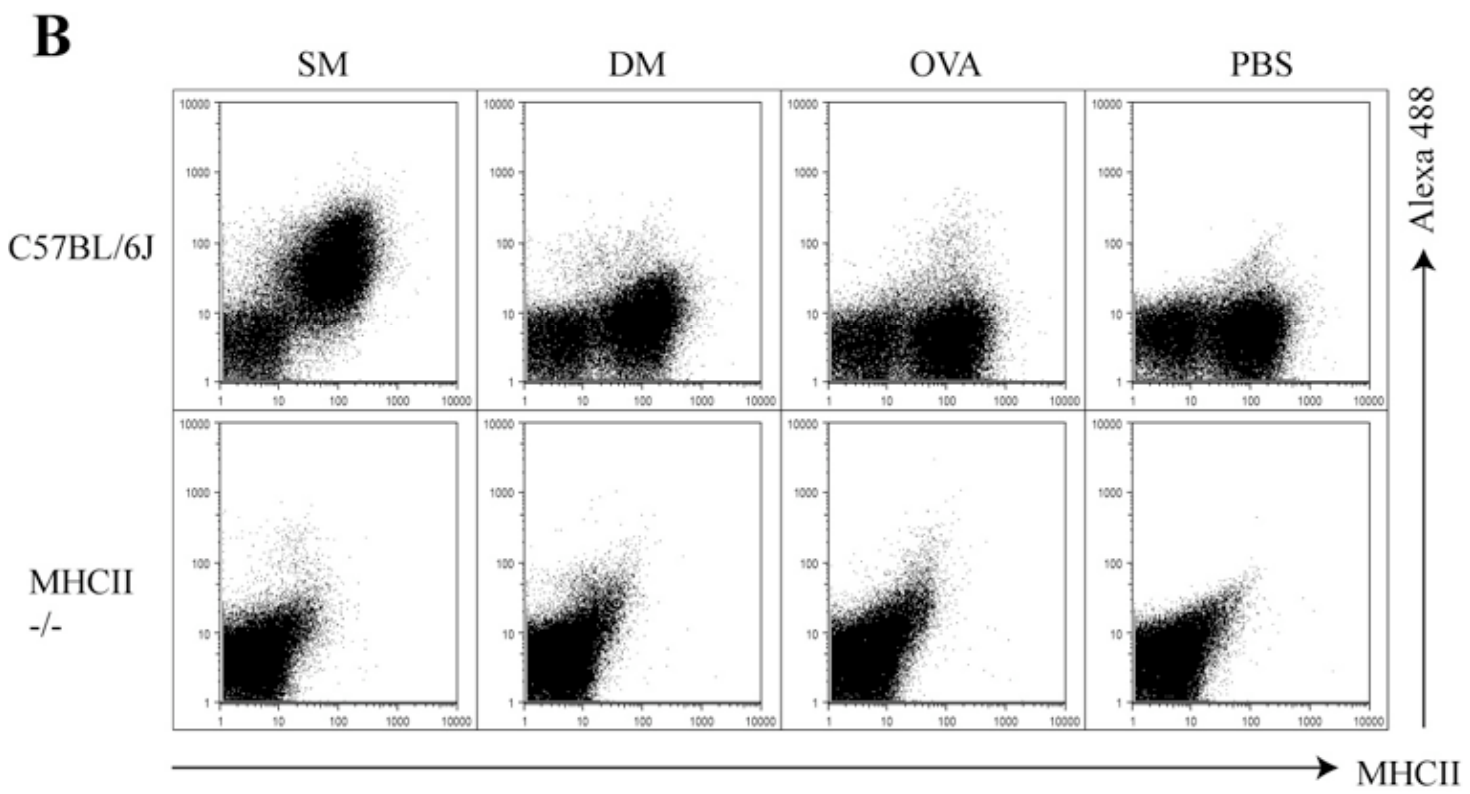

Figure 4.1 MHCII ${ }^{+}$cells did not stain with DM both in vitro and in vivo

WT, SM, DM or OVA proteins were labelled with Alexa 488 dye. A, In vitro, $\mathrm{MHCII}^{+}$cells did not stain with DM. The Alexa 488 labelled proteins or PBS as indicated were incubated with C57BL/6J mouse splenocytes at room temperature for 30 minutes $(0.02 \mathrm{mg} / \mathrm{ml} \mathrm{mSAg}$-Alexa 488 and $0.03 \mathrm{mg} / \mathrm{ml}$ OVA-Alexa 488). After incubation, the cells were analysed by flow cytometry for the staining of the indicated proteins. B, In vivo, $\mathrm{MHCII}^{+}$cells did not stain with DM. The Alexa 488 labelled proteins or PBS as indicated were injected into either C57BL/6J or MHCII-/- mice i.v. (50 $\mu \mathrm{g}$ of mSAg-Alexa 488 per animal and $75 \mu \mathrm{g}$ of OVA-Alexa 488). Three hours after the injections, the splenocytes from the injected mice were collected and analysed by flow cytometry for the staining of the indicated proteins. Results are representative of more than three separate experiments. 
4.3.1.2 Conjugation of DM to peptide did not enhance the peptide presentation to CD4+ cells

As shown in the previous chapter, the conjugation of SM to the peptide known to be presented by MHCII greatly enhanced peptide presentation to $\mathrm{CD}^{+} \mathrm{T}$ cells. To determine whether DM has lost this enhanced peptide presentation to $\mathrm{CD}^{+} \mathrm{T}$ cells due to a lack of MHCII binding, I conjugated DM with the peptides $\mathrm{MOG}_{35-55 \mathrm{c}}$ and OVA $_{323-339 c}$ (Figure 4.2A) and compared DM-peptide and SM-peptide in peptide presentation to $\mathrm{CD} 4^{+} \mathrm{T}$ cells.

In vitro $\mathrm{T}$ cell proliferation assays were performed to assess the mSAg-peptide's efficiency for the induction of $\mathrm{CD}^{+} \mathrm{T}$ cell proliferation. Different concentrations of conjugated or unconjugated peptides were incubated with splenocytes from either 2D2 or OTII transgenic mice. As shown in Figure 4.2B, approximately 100-1,000 fold less SM-peptide was required to achieve the levels of the $\mathrm{CD} 4^{+} \mathrm{T}$ cell proliferation observed with DM-peptide or non-conjugated peptide. This result provided further evidence that $\mathrm{DM}$ did not enhance peptide presentation to $\mathrm{CD} 4^{+} \mathrm{T}$ cells and that $\mathrm{DM}$ did not bind to MHCII. In summary, DM was unable to bind to MHCII and the conjugation of DM to peptides did not enhance the presentation of these peptides to $\mathrm{CD} 4^{+} \mathrm{T}$ cells. 


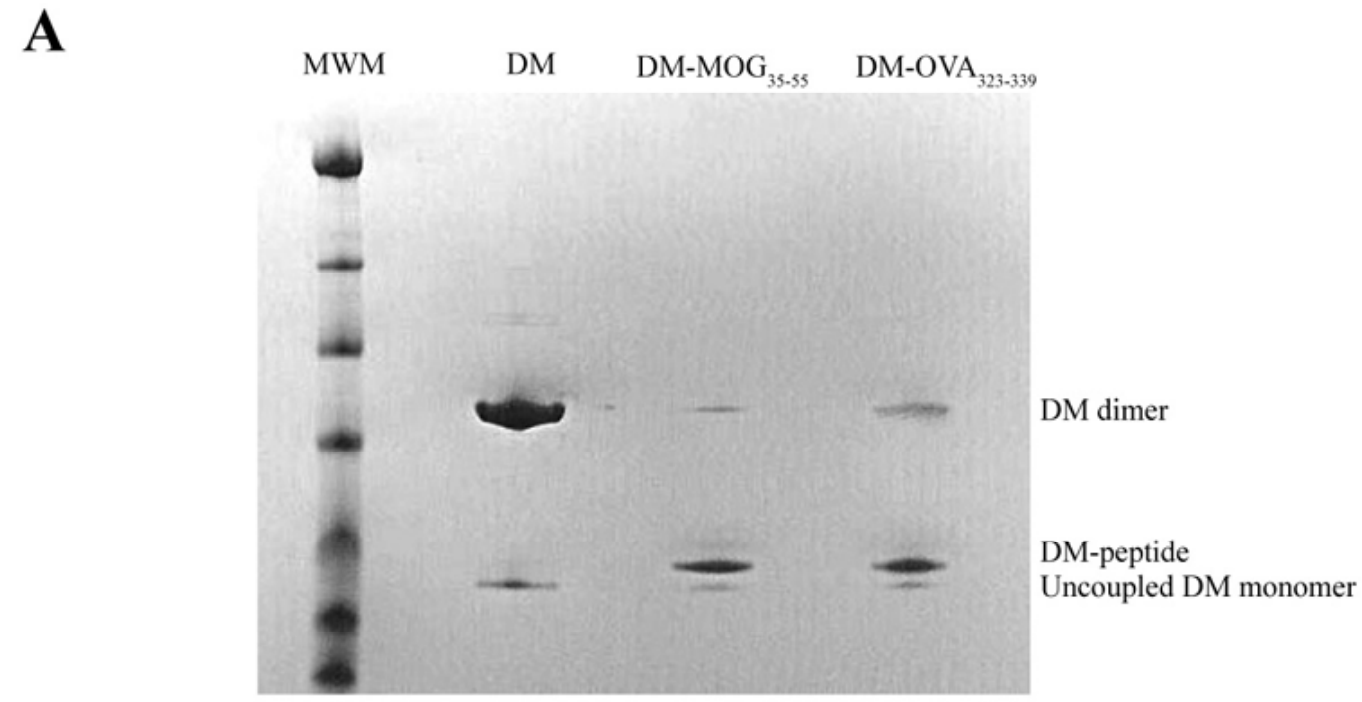

B

2D2

$\left(\mathrm{MOG}_{35.55}\right.$ specific)
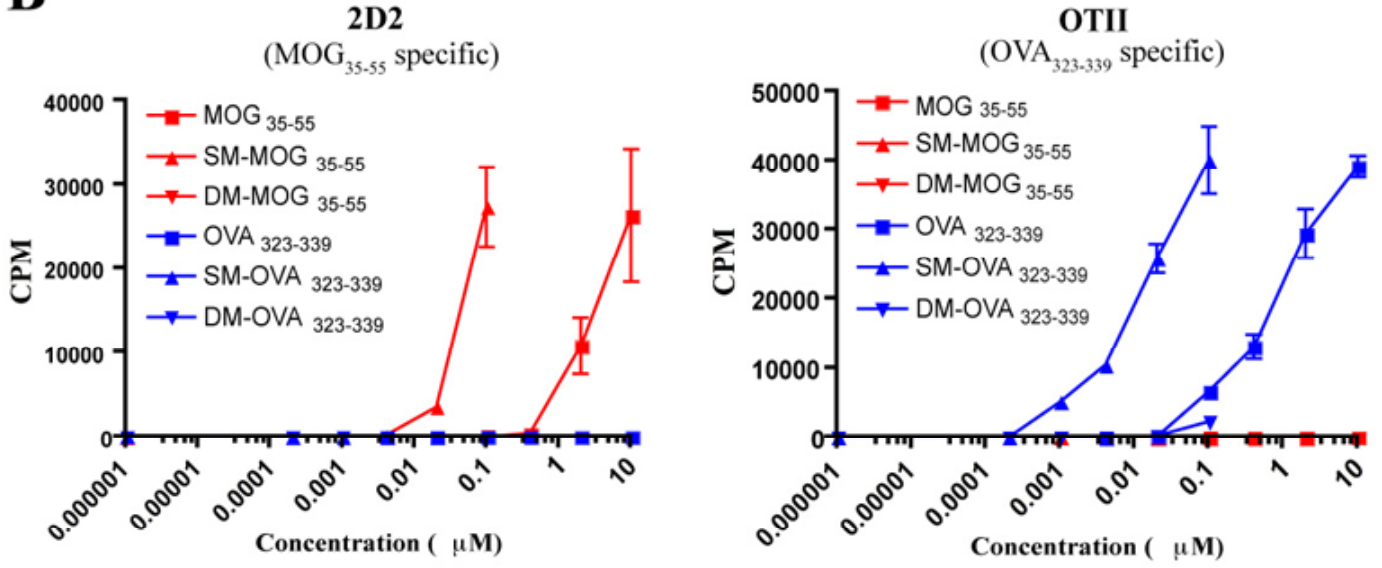

Figure 4.2 The peptide conjugated to DM and SM were differently presented to $\mathrm{CD4}^{+} \mathrm{T}$ cells in vitro

A, DM was conjugated with either the $\mathrm{MOG}_{35-55 \mathrm{c}}$ or $\mathrm{OVA}_{323-339 \mathrm{c}}$ peptide and the conjugation was assessed by a NuPage gel under a non-reducing condition. MWM: molecular weight marker. B, in vitro proliferation assay with the splenocytes from either 2D2 or OTII transgenic mice. The splenocytes were incubated with the indicated concentrations of antigens at $1 \times 10^{6}$ cells per well. Cell proliferation was assayed by measuring the incorporation of $\left[{ }^{3} \mathrm{H}\right]$-thymidine over the last 16 hours of a 72 -hour culture period. Due to limited availability of SM and DM, the doses of mSAg-peptides were used up to $0.1 \mu \mathrm{M}$. Results are shown as the mean \pm SEM of triplicate wells. Results are representative of more than three separate experiments.

\subsubsection{DM bound to the $\mathrm{MHCII}^{-} \mathrm{CD} 11 \mathrm{~b}^{+} \mathrm{F} 4 / \mathrm{80}^{+} \mathrm{Gr}-1^{+}$blood cells in vivo}

In order to verify whether DM also bound to the same $\mathrm{SM}^{+} \mathrm{MHCII}^{-}$blood cells as described in Chapter 3, Alexa 488 labelled DM was injected i.v. into C57BL/6J mice. SM-Alexa 488, OVA-Alexa 488 or PBS was injected into mice in parallel. Three hours after the injection, cells from the lymph nodes, spleens and blood of these mice were collected and analysed by flow cytometry. As shown in Figure 4.3A, DM staining was observed on a $\mathrm{MHCII}^{-}$cell population in the blood and this population was larger than 
the $\mathrm{SM}^{+} \mathrm{MHCII}$ population. This result was consistent with the fact that SM preferentially binds to MHCII molecules and thus most SM binds to $\mathrm{MHCII}^{+}$cells in vivo.

DM and SM also stained on this blood cell population in MHCII deficient (MHCII-/-) mice, indicating that the uptake of the mSAgs by these blood cells was independent of MHCII molecules. This uptake was not a result of antigen-non-specific phagocytosis, as in the mice injected with the same molar dose of OVA-Alexa 488 only marginal staining was observed. This observation reveals a possible unique MHCII-independent uptake mechanism for superantigen strain SMEZ-2.

DM-Alexa 488 staining was present but marginal in the spleens and absent in other tissues, such as the lymph node, spinal cord and liver e.g. Figure 4.3B. No staining was observed when DM-Alexa 488 was injected into mice s.c. (in IFA, CFA or PBS) or i.p. (in IFA or CFA). 
A

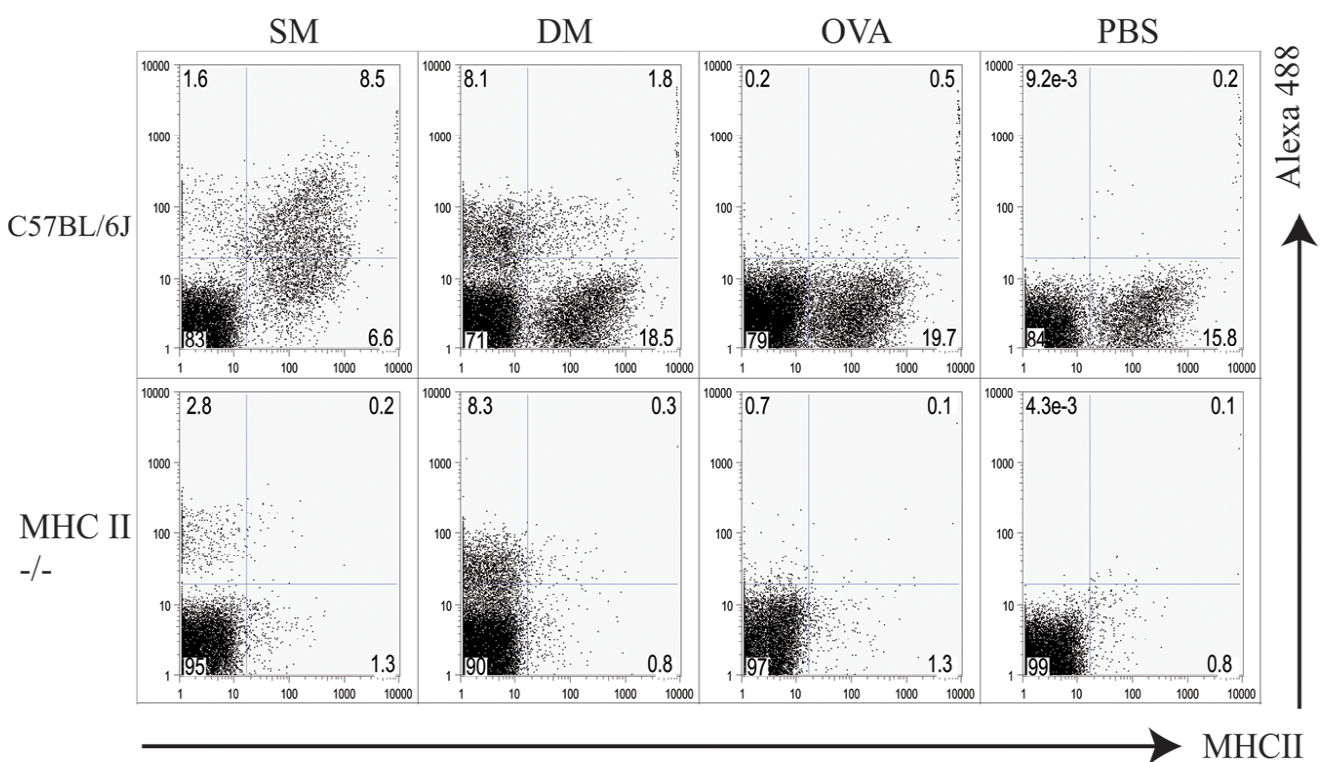

B

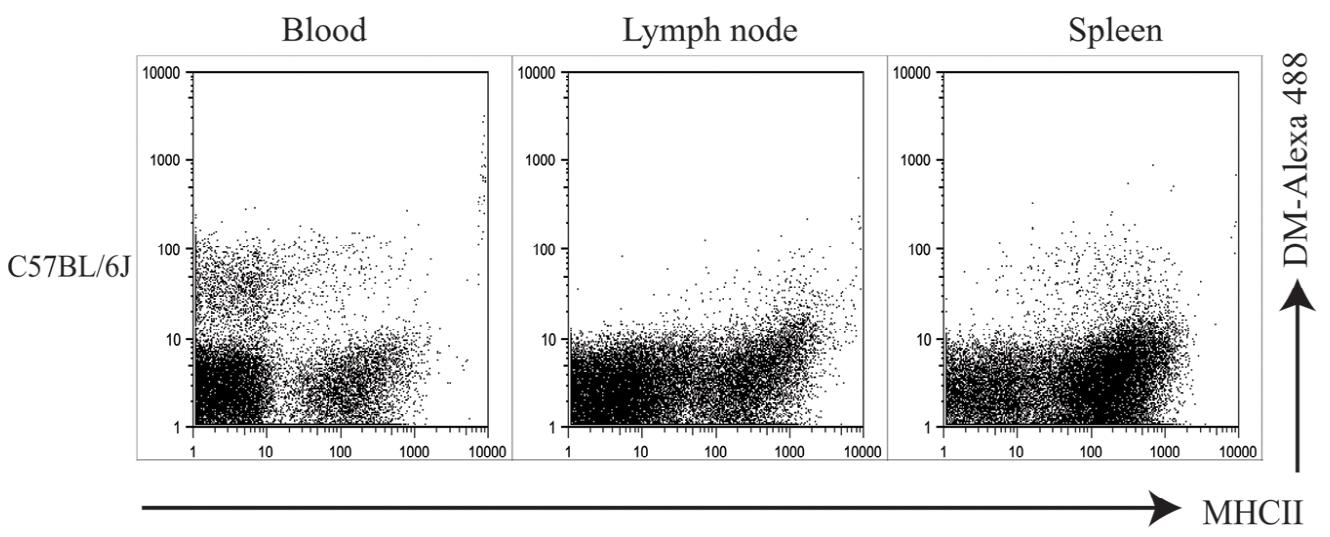

Figure 4.3 A blood-borne cell population stained with SM- and DM-Alexa 488

C57BL/6J and MHCII-/- mice were injected i.v. with $50 \mu \mathrm{g}$ SM-Alexa 488, DM-Alexa 488, $75 \mu \mathrm{g}$ OVA-Alexa 488 or PBS respectively. Three hours after the injections, cells from the blood, spleens and lymph nodes were collected and analysed. A, Alexa 488 staining on the blood cells from the mice injected with the indicated Alexa 488 labelled proteins. B, Alexa 488 staining on the blood cells, lymph node cells and splenocytes derived from the DM-Alexa 488 injected mice. Numbers indicate percent of total cells in each quadrant. Results are representative of more than three separate experiments.

\subsubsection{The binding to the blood MHCII- cells was not specific to superantigen}

In order to understand whether the MHCII independent uptake of superantigens was specific for the strain SMEZ-2, I injected C57BL/6J mice i.v. with another strain of superantigen, streptococcal pyrogenic exotoxin C (SPEC). The primary target for SPEC is the I-E molecule ${ }^{195}$. C57BL/6J mice lack expression of the I-E $\beta$ chain ${ }^{173}$, so SPEC is unable to interact with MHCII in these mice. Like DM, SPEC was also taken up by the blood MHCII- cell population in vivo (Figure 4.4A). In contrast, only marginal staining was observed in the mice injected with the Alexa 488 labelled control proteins, OVA (Figure 4.3A) or MOG (Figure 4.4B). These results indicate that the 
MHCII-independent protein uptake by the blood cells is not specific for the superantigen SMEZ-2 strain.

To exclude the possibility that protein size was important for the observed difference in uptake of superantigens $(25 \mathrm{KDa})$, OVA $(40 \mathrm{KDa})$ and MOG $(12 \mathrm{KDa})$, an unrelated protein trypsinogen (Sigma-Aldrich, USA) that had a similar molecular weight to DM of $\sim 25 \mathrm{KD}$ was labelled with Alexa 488 and injected into mice. As shown in Figure $4.4 \mathrm{C}$, trypsinogen was also taken up by the $\mathrm{MHCII}^{-}$blood cells. Therefore, the uptake of the proteins by these MHCII- blood cells is not superantigen-specific. Based on this finding, a separate project in our laboratory was carried out to investigate the potential mechanisms of the association between the above proteins and the blood cells (Mirmoeini S., unpublished data).

(A) SPEC

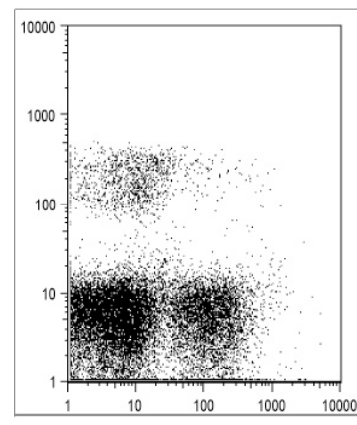

(B) MOG

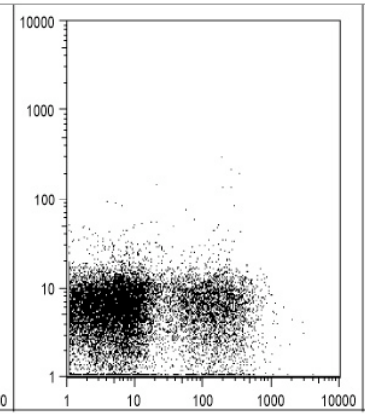

(C) Trypsinogen

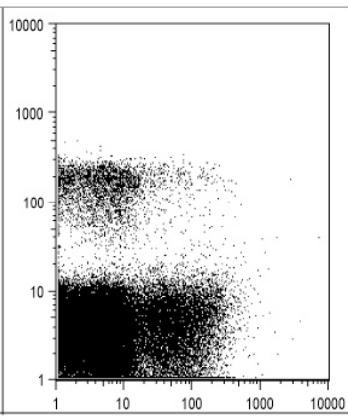

(D) DM

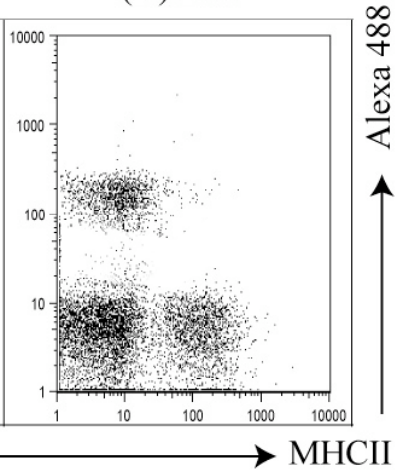

Figure 4.4 The binding to the blood $\mathrm{MHCII}^{-}$cells was not specific to superantigen C57BL/6J mice were injected i.v. with $50 \mu \mathrm{g}$ SPEC, MOG protein, trypsinogen or DM labelled with Alexa 488. Three hours after the injections, cells from the blood were collected and analysed by flow cytometry. Results are representative of two separate experiments.

\subsubsection{The $\mathrm{DM}^{+}$cells were myeloid cells}

The blood cells that bound to DM made up 5-20\% of all the leukocytes and $\sim 1-2 \%$ of the splenocytes in mice. The blood $\mathrm{DM}^{+}$cells demonstrated strong staining of CD11b, CD11a, CD45, CD49d and intermediate levels of Gr-1, F4/80 and CD80 staining (Table 4.1), whereas no CD86, MHCII, PDCA-1 or any classic B or T cell markers were detected on them. These $\mathrm{DM}^{+}$blood cells were heterogenous in their Ly6C expression, ranging from negative to high, but were negative for Ly6G expression, indicating that these cells were not neutrophils ${ }^{71,196,197}$. The expression of the classic myeloid cell surface markers such as F4/80 and CD11b on these $\mathrm{DM}^{+}$cells indicated their myeloid lineage. The $\mathrm{DM}^{+}$cells also present a myeloid-like morphology with a large, ovoid or 
kidney-shaped nucleus (Figure 4.5A), and DM was taken into cytoplasm rapidly after i.v. injection (within 20 minutes) (Figure 4.5B). Together with the fact that this uptake was not dependent on MHCII, it suggests the existence of a unique receptor for SMEZ-2 on these blood-borne cells.

Table 4.1 The surface staining of $\mathrm{DM}^{+} \mathrm{CD}_{11}{ }^{+}$cells in the blood

\begin{tabular}{ll}
\hline Myeloid lineage markers & + \\
\hline F4/80 & + \\
\hline Monocyte subset markers &,,-+++ \\
\hline Ly6C & - \\
Ly6G & + \\
Gr-1 & \\
\hline Adhesion molecules & ++ \\
\hline CD11a (LFA-1) & ++ \\
CD11b (MAC-1) & ++ \\
CD44 & - \\
CD54 (ICAM-1) & ++ \\
CD49d (VLA-4) & - \\
\hline Antigen presentation/costimulatory markers &,-+ \\
\hline MHCII & - \\
CD80 &,-+ \\
CD86 & - \\
CD40 & - \\
\hline Miscellaneous & - \\
\hline CD90.2 (Thy1.2) & - \\
CD4 & - \\
CD8 & - \\
CD45 & - \\
CD45R (B220) & - \\
CD62L & - \\
CD69 & - \\
24G2 & - \\
CD11c & - \\
CDCA-1 & - \\
\hline
\end{tabular}

Data have been assigned arbitrary symbols that represent "-" no staining and increasing amount of staining “,$+++"$. 
A
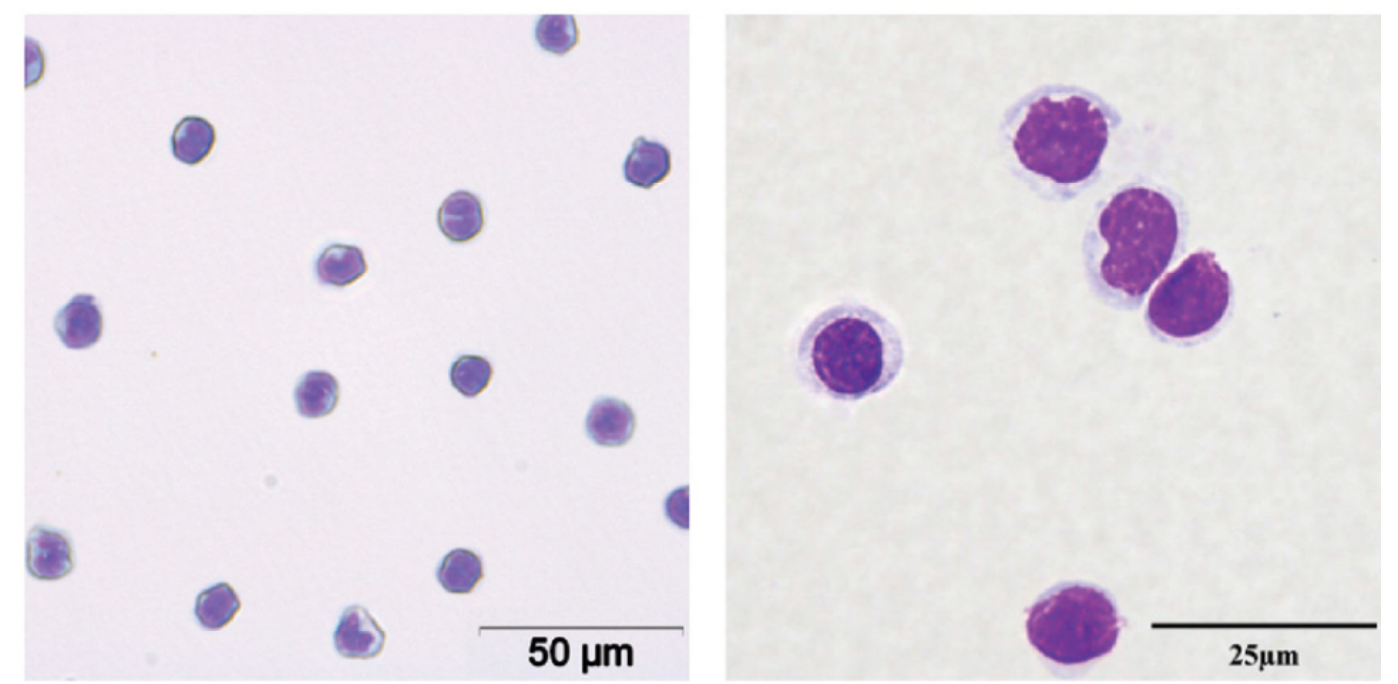

B
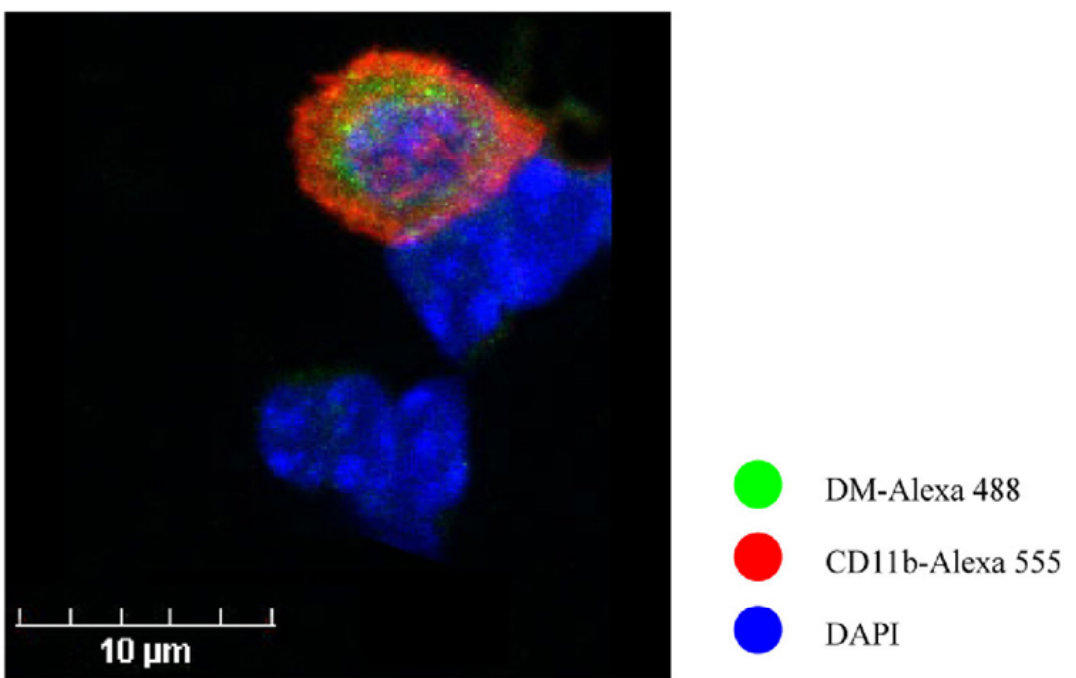

Figure 4.5 DM was taken into the cytoplasm of a myeloid cell population in the blood

C57BL/6J mice were injected with DM-Alexa 488 i.v. (50 $\mu$ g per mouse). Twenty minutes after injection, the blood cells were isolated. A, DM-Alexa $488^{+}$blood cells presented a myeloid-like morphology with a large, ovoid or kidney-shaped nucleus. Left image, DM-Alexa $488^{+}$cells were viewed by microscopy under a 400 times magnification and right image, 1000 times magnification. $100 \%$ pure DM-Alexa $488^{+}$cells were sorted by FACS sorting and stained by using a Diff-Quik stain set. B, Confocal microscopy image of the blood cells collected 20 minutes after i.v. injection of Alexa 488 labelled DM. The co-staining of CD11b (surface marker) and DAPI (intracellular marker) indicated that DM was located in the cytoplasm.

\subsubsection{The blood $\mathrm{MHCIICD} 11 \mathrm{~b}^{+} \mathrm{F} 4 / \mathrm{80}^{+} \mathrm{Gr}-1^{+}$cells were potent suppressors of $\mathrm{T}$ cell} response

The $\mathrm{DM}^{+}$cells identified in the blood expressed several features common to the MDSC phenotypes, including their monocytic morphology and co-expression of Gr-1, CD11b and $\mathrm{F} 4 / 80^{45}$, thereby raising the question whether these blood cells also exhibited a suppressor function. 


\subsubsection{1 $\mathrm{DM}^{+}$bound to the $\mathrm{CD}^{11 \mathrm{~b}^{+}}{ }^{\mathrm{Ly} 6 \mathrm{G}^{-} \mathrm{SSC}^{\text {low }}}$ cells in the blood}

In the mice injected with DM-Alexa 488 , all the $\mathrm{DM}^{+}$cells in the blood had a

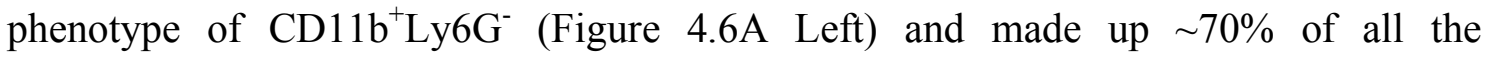
$\mathrm{CD}_{11 \mathrm{~b}^{+} \mathrm{Ly}_{6}}^{-}$cells in the blood (Figure 4.6A Right). The CD11b ${ }^{+} \mathrm{Ly}_{6 \mathrm{G}}^{-}$cells were present in the naïve mouse blood with a similar percentage (Figure 4.6B) and expressed a similar pattern of surface markers as listed in Table 4.1. It is noteworthy that the CD11 b ${ }^{+}$Ly6G $^{-}$cells in the mouse blood were located in the low side-scatter gate whereas the $\mathrm{CD} 11 \mathrm{~b}^{+} \mathrm{Ly}_{6 \mathrm{G}^{+}}$neutrophils were located in the high side scatter gate (Figure 4.7). This result indicated that the $\mathrm{CD}_{11} \mathrm{~b}^{+} \mathrm{Ly} 6 \mathrm{G}^{-}$cells had lower cellular granularity than neutrophils, and this finding is consistent with the morphological characteristics shown in Figure 4.5B.

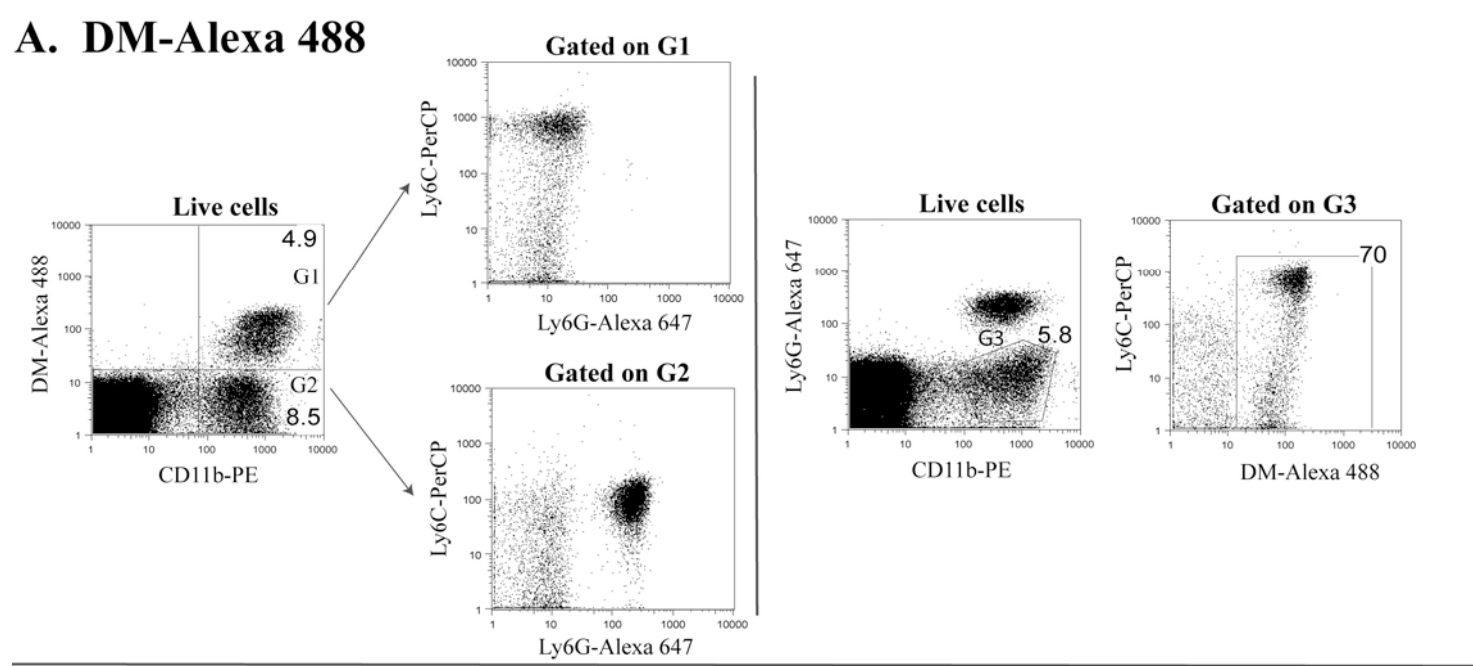

\section{B. PBS}
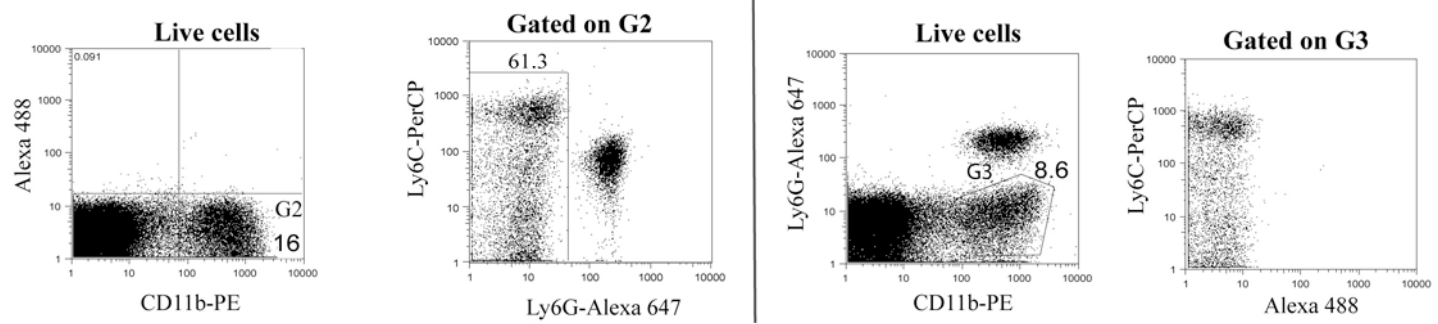

Figure 4.6 The $\mathrm{DM}^{+}$cells were $\mathrm{CD} 11 \mathrm{~b}^{+} \mathrm{Ly6G}^{-}$

DM-Alexa 488 was injected i.v. into a C57BL/6J mouse with a dose of $50 \mu \mathrm{g}$. Thirty minutes after injection, the blood cells were collected and analysed. An age- and sex-matched mouse was injected with PBS as the experimental control. A, the blood cells from the mouse injected with DM-Alexa 488. Left,

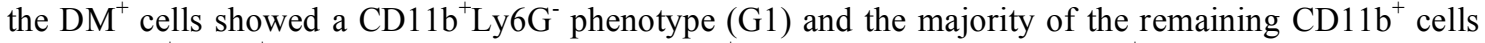
were $\mathrm{Ly}_{6 \mathrm{G}}^{+} \mathrm{Ly}_{6 \mathrm{C}}^{+}(\mathrm{G} 2)$. Right, $70 \%$ of the $\mathrm{CD} 11 \mathrm{~b}^{+} \mathrm{Ly}_{6 \mathrm{G}}{ }^{-}$blood cells were $\mathrm{DM}^{+}$. B, the blood cells from the control mouse. The numbers indicate the percentages of gated cells. 

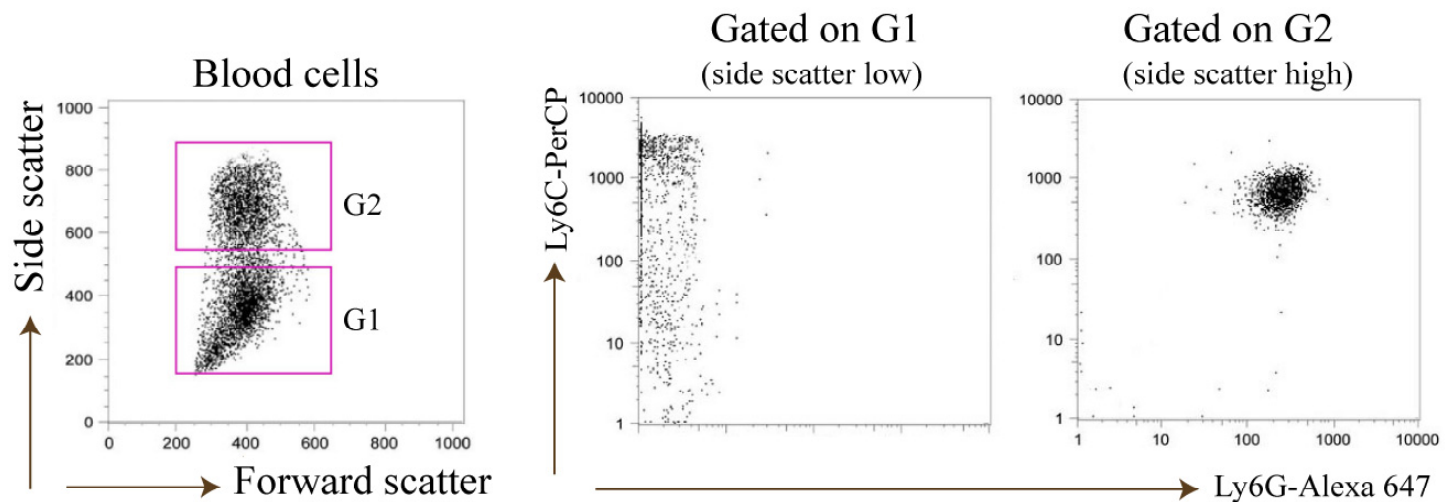

Figure 4.7 The CD11 ${ }^{+} \mathrm{Ly}^{-} \mathrm{G}^{-}$cells had a low side scatter intensity profile

Left graph, the blood cells from naïve mice were analysed by flow cytometry. G1 and G2 were gated on side-scatter high and low cells. Right graph, CD1 $1 b^{+}$cells located in G1 and G2 were analysed for the expression of Ly6C and Ly6G. The cells of the right graph were gated on $\mathrm{CD} 11 \mathrm{~b}^{+}$cells.

\subsubsection{2 $\mathrm{CD}^{11 \mathrm{~b}^{+}} \mathrm{Ly6G}^{-}$cell purification from the blood}

In order to obtain pure cell populations for functional assays, different cell purification methods were tested. The traditional method for cell purification is to use antibodies in combination with magnetic beads to positively select out the desired cell population. However, this approach was not possible for the $\mathrm{CD} 11 \mathrm{~b}^{+} \mathrm{Ly}_{6 \mathrm{G}^{-}}$cell population as the markers expressed by the $\mathrm{DM}^{+}$cells such as $\mathrm{CD} 11 \mathrm{~b}$, Gr-1 and Ly6C were also expressed on other cells including the $\mathrm{Ly}_{6 \mathrm{G}}^{+}$neutrophils. As a result, the $\mathrm{Ly}_{6 \mathrm{G}}^{+}$ neutrophils were required to be removed before enrichment of $\mathrm{CD} 11 \mathrm{~b}^{+}$cells.

Because the CD11b ${ }^{+}$Ly6G- cells have different granularity (Figure 4.7), a density gradient was used to eliminate the $\mathrm{Ly}_{6} \mathrm{G}^{+}$neutrophil population. Three density gradients were trialed, Percoll (Amersham Biosciences, Sweden), Histopaque 1083 (Sigma-Aldrich, USA) and Lympholyte-M (Cedarlane Ltd., USA). Of these three, Lympholyte-M provided the best performance for eliminating $\mathrm{Ly}_{6 \mathrm{G}}{ }^{+}$neutrophils (from $12 \%$ to $<1 \%$ ) (Figure 4.8 ) and high recovery of the $\mathrm{CD} 11 \mathrm{~b}^{+} \mathrm{Ly} 6 \mathrm{G}^{-}$cells.

After removal of $\mathrm{Ly}_{6 \mathrm{G}}{ }^{+}$neutrophils, a combination of $\mathrm{CD} 11 \mathrm{~b}^{+}$antibody with magnetic beads was used to positively select the $\mathrm{CD}_{11 \mathrm{~b}^{+} \mathrm{Ly} 6 \mathrm{G}^{-}}$cells. I compared the combinations of the $\mathrm{CD} 11 \mathrm{~b}$ antibody with three different magnetic cell separation systems including MACS (Miltenyi Biotec, Germany), Dynal (Invitrogen, USA) and Biomag (Bangs Laboratories Inc., USA). The MACS system exhibited the best performance with the $\mathrm{CD} 1 \mathrm{~b}^{+} \mathrm{Ly} 6 \mathrm{C}^{-}$cells being enriched to $80-90 \%$. This cell purification procedure is described in detail in Section 2.7.1.3. In conclusion, by using 
this two-step purification method, Lympholyte-M followed by CD11b antibody and MACS beads, the CD $11 b^{+}{\text {Ly } 6 G^{-}}^{-}$blood cells were enriched from $5-25 \%$ to $>80 \%$.

The purification method above was then used to determine the \%purified CD11 ${ }^{+} \mathrm{Ly}_{6 \mathrm{G}}^{-}$ cells that were $\mathrm{DM}^{+}$cells in the blood of DM-Alexa 488 injected mice. Approximately $70 \%$ of the purified cells were $\mathrm{DM}^{+}$cells (Figure 4.9 ). This number was consistent with

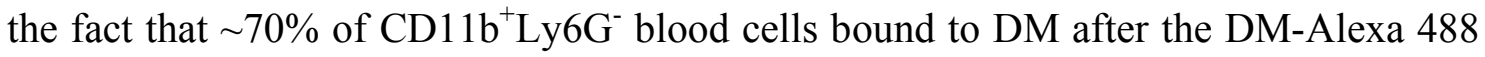
i.v. injection (Figure 4.6). Therefore, this two-step purification is an efficient method to purify the $\mathrm{CD} 11 \mathrm{~b}^{+} \mathrm{Ly}_{6 \mathrm{G}^{-}}$cells from the blood.

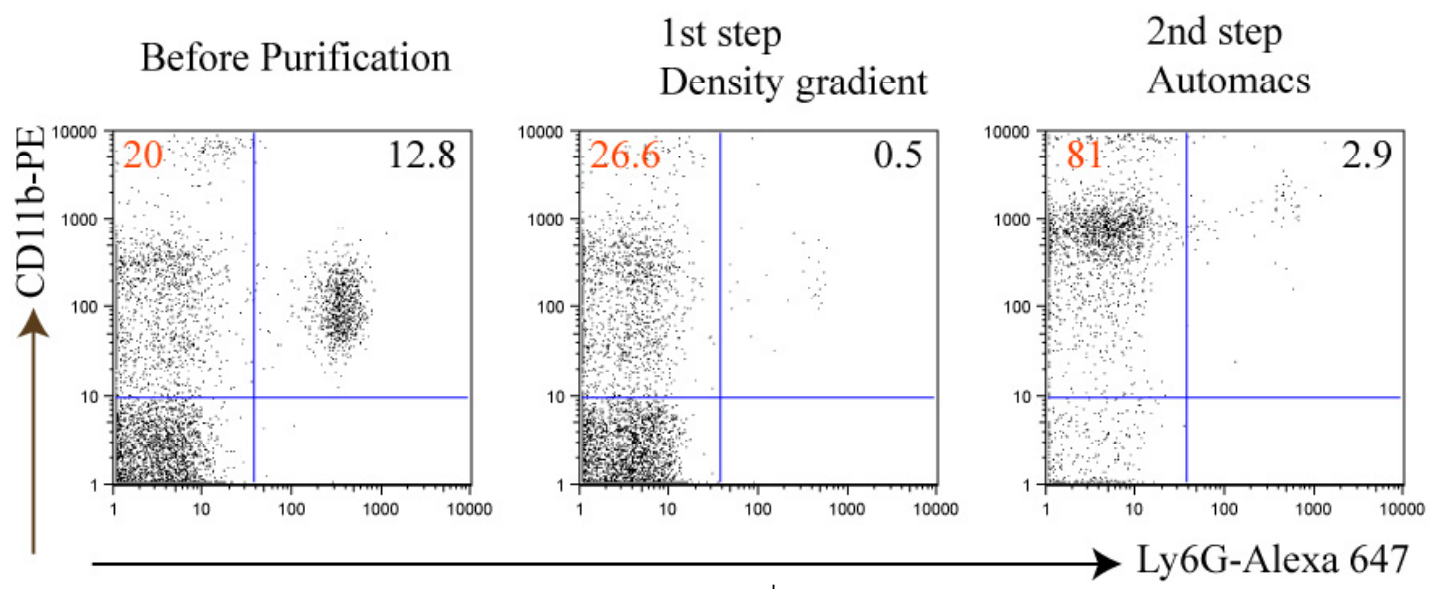

Figure 4.8 Two-step purification of $\mathrm{CD}^{11 b^{+}} \mathrm{Ly6G}^{-}$cells from the blood

A two-step purification method was established for the blood $\mathrm{CD} 11 \mathrm{~b}^{+} \mathrm{Ly} 6 \mathrm{G}^{-}$cell enrichment. In the first step, whole blood cell suspension was centrifuged with Lympholyte-M density gradient to eliminate the Ly $6 \mathrm{G}^{+}$neutrophils. In the second step, $\mathrm{CD} 11 \mathrm{~b}^{+} \mathrm{Ly} 6 \mathrm{G}^{-}$cells were positively selected by $\mathrm{CD} 11 \mathrm{~b}$ antibody in combination with MACS beads through an Automacs separator. The numbers indicate the percentages of the gated cells. The numbers in red indicate the percentages of target CD11 b ${ }^{+}$Ly6G $\mathrm{G}^{-}$blood cells in the cell suspension.

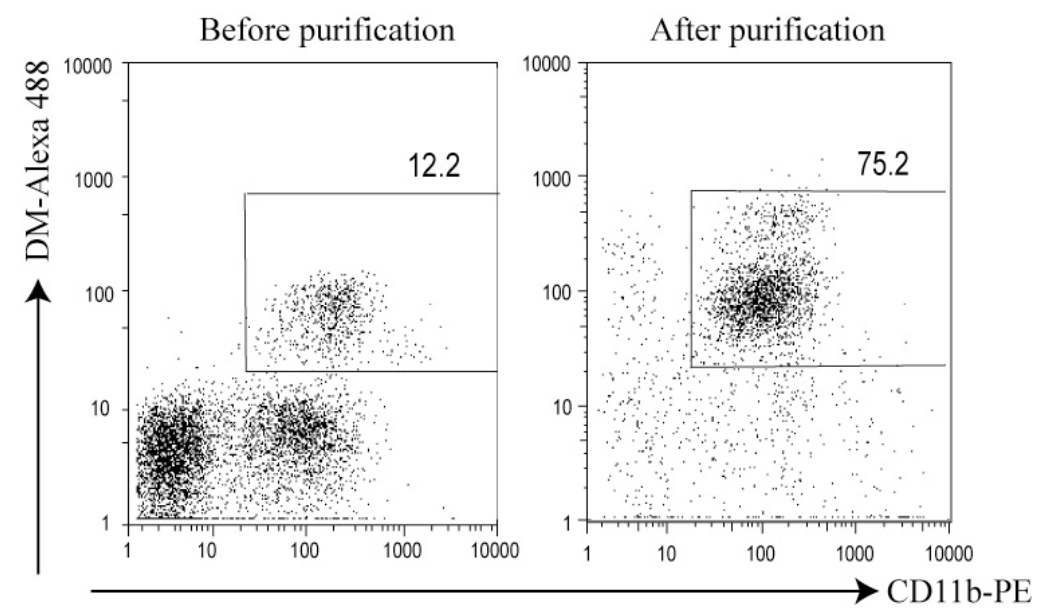

Figure 4.9 Purification of the $\mathrm{DM}^{+}$cells via the two-step purification procedure

DM-Alexa 488 was injected i.v. into a C57BL/6J mouse with a dose of $50 \mu \mathrm{g}$. Blood cells were isolated from the mouse three hours after the injection. Left, the original blood population; Right, after the two-step purification, $>70 \%$ of the final cell population were $\mathrm{DM}^{+}$cells. 


\subsubsection{Validation of a suppression assay for measuring the blood $\mathrm{CD}^{11 b^{+}} \mathrm{Ly}^{6} \mathrm{G}^{-}$ cells-mediated suppressive effect}

To determine whether the blood $\mathrm{CD} 11 \mathrm{~b}^{+} \mathrm{Ly} 6 \mathrm{G}^{-}$cells expressed a suppressive

phenotype, the purified blood $\mathrm{CD} 11 \mathrm{~b}^{+} \mathrm{Ly}_{6 \mathrm{G}}^{-}$cells were tested for their ability to suppress $\mathrm{T}$ cell proliferation in vitro using a traditional $\mathrm{T}$ cell suppression assay (described in Section 2.11.2.1). The assay involved stimulating the responder T cells in the presence of the proposed suppressor blood CD11 b ${ }^{+}$Ly $6 \mathrm{G}^{-}$cells. $\left[{ }^{3} \mathrm{H}\right]$-thymidine was added to the cell culture and incubated with the cells for a further 8-16 hours before the termination of the assay. The suppression of $\mathrm{T}$ cell proliferation was measured by the inhibition of thymidine incorporation.

Surprisingly, in many experiments, the suppression of cell proliferation was evident under a microscope (Figure 4.10A), but this suppression could not be detected by thymidine incorporation (Figure 4.10B). In order to investigate the reason for the conflicting results measured by microscopy and thymidine incorporation, and develop a reliable assay to evaluate the suppressive effect of the blood $\mathrm{CD} 11 \mathrm{~b}^{+} \mathrm{Ly}_{6 \mathrm{G}}^{-}$cells, I assessed $\mathrm{T}$ cell proliferation by CFSE dilution assay. In contrast to microscopy and thymidine incorporation, CFSE dilution captures difference in cell divisions from time point zero to the end of the assay ${ }^{188}$. As shown in Figure $4.10 \mathrm{C}$, using the same conditions described in Figure 4.10A and Figure 4.10B, the suppression of responder cell division was evident by measuring CFSE dilution. The suppression measured by CFSE dilution was in a clear dose-dependent manner, which was consistent with the effect when observed by microscopy (Figure 4.10A). 
A
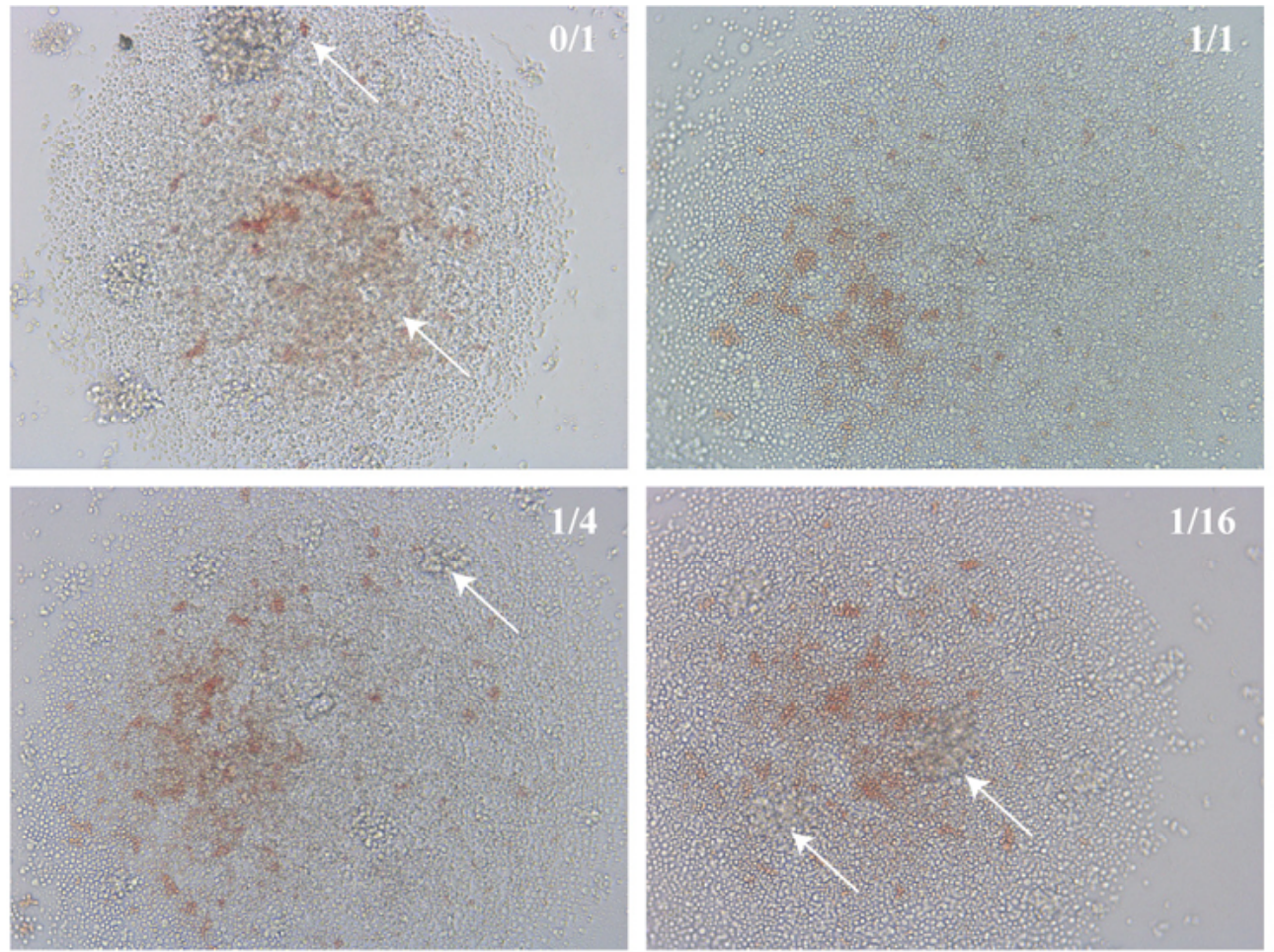

B

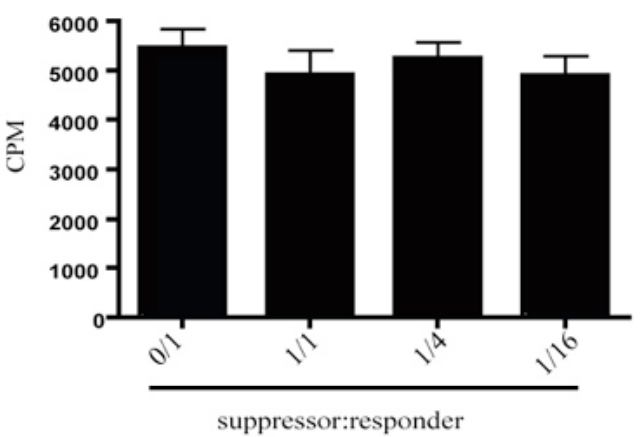

responter cells tmedium only

$\mathrm{C}$
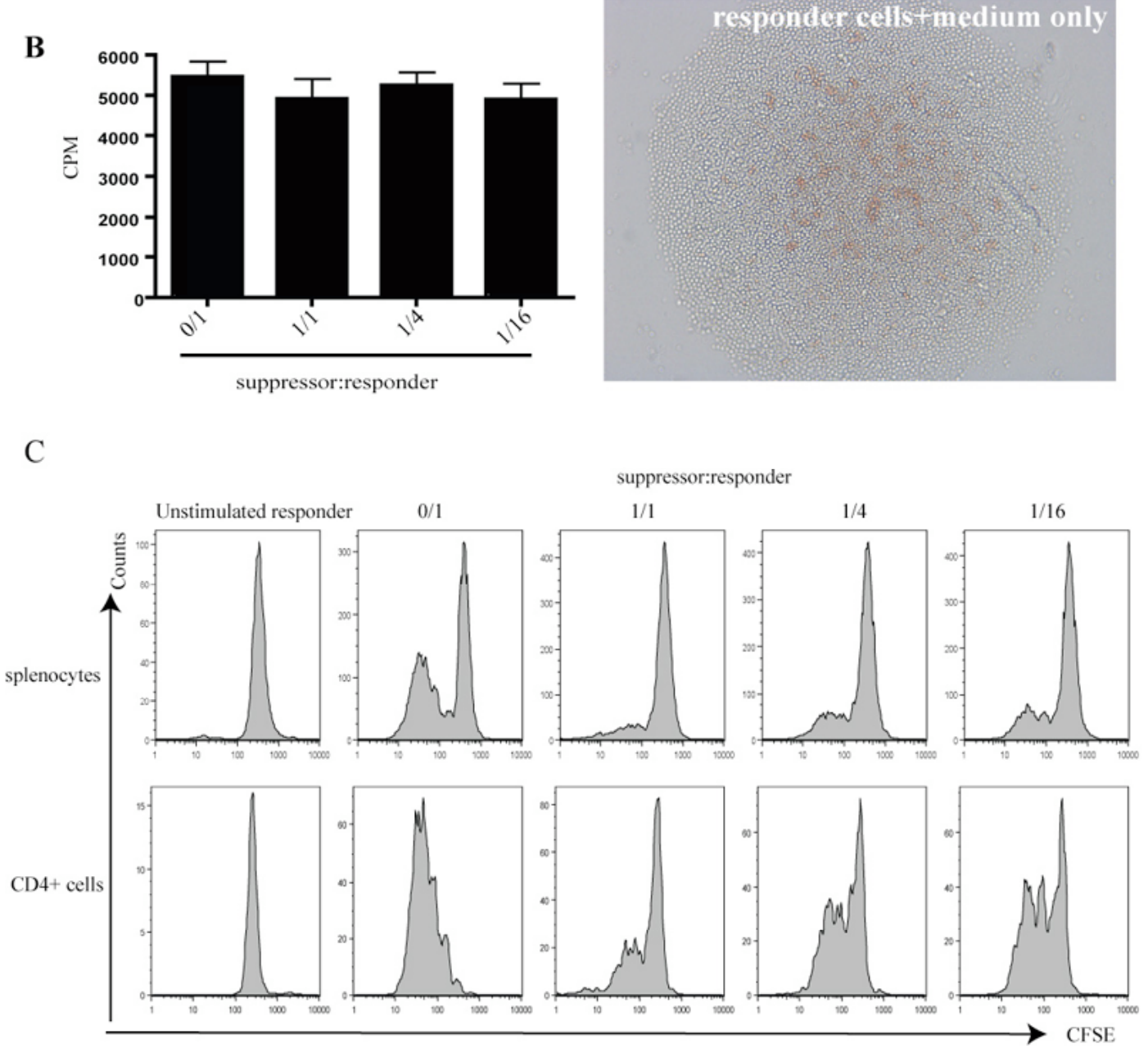

Figure 4.10 Comparison of thymidine incorporation and CFSE dilution based suppression assays

CFSE labelled 2D2 transgenic splenocytes were used as the responder cells and pre-incubated with $10 \mu \mathrm{g} / \mathrm{ml} \mathrm{MOG}_{35-55}$ for 24 hours. Purified blood CD11 b ${ }^{+} \mathrm{Ly}_{6} \mathrm{G}^{-}$cells were added to the cell culture at the 
indicated ratios to the responder cells and incubated for a further 40 hours. A, Microscopy pictures taken at 64 hours after the initiation of the cell culture. The suppression of the splenocyte proliferation by the blood $\mathrm{CD} 11 \mathrm{~b}^{+} \mathrm{Ly}_{6 \mathrm{G}^{-}}$cells was evident under a microscope and this suppression was dose dependent. Arrows indicate the proliferating cells formed clusters. The numbers indicate the ratios of suppressor:responder cells. B, The cells of graph A were tested for thymidine uptake. C top, CFSE dilution on overall responder cells. $\mathbf{C}$ bottom, CFSE dilution on gated $\mathrm{CD}^{+}$cells. Eight to 16 hours before the termination of the assay, $\left[{ }^{3} \mathrm{H}\right]$-thymidine was added to the cell culture. When the thymidine plate was harvested (B), the duplicate plate was analysed for CFSE dilution. Note in $\mathbf{C}$, the dose-dependent suppression was obvious but did not show in $\mathbf{B}$.

As the CFSE and thymidine based assays presented different readouts of the blood CD11b ${ }^{+}$Ly6G $^{-}$cells' suppressive activity (Figure 4.10), I hypothesised that the suppression of $\mathrm{T}$ cell proliferation by the blood $\mathrm{CD}_{11} \mathrm{~b}^{+} \mathrm{Ly}_{6 \mathrm{G}}^{-}$cells only lasted for a short period of time. Based on this theory, it was possible that before thymidine was added to the cell co-culture, this suppressive activity had already diminished. In contrast, CFSE dilution detected the suppression of cell division from time zero to the end of the assay, and therefore, was able to detect any difference in $\mathrm{T}$ cell proliferation before the addition of thymidine.

To test whether timing was important for the thymidine incorporation assay in detecting

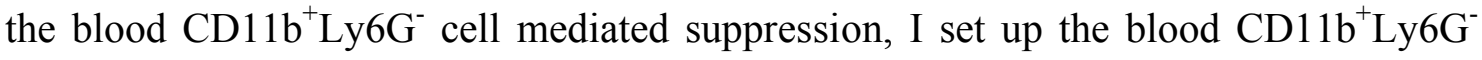
cell and $\mathrm{CD}^{+}$cell co-cultures and performed the thymidine incorporation assays at different time points. As shown in Figure 4.11, the blood CD11b ${ }^{+}$Ly $_{6} \mathrm{G}^{-}$cells' suppressive activity was maximal on day one and was absent by day three, indicating that the cell proliferation was suppressed only during the early phase of $\mathrm{T}$ cells expansion. This finding supports the above hypothesis.

Thymidine incorporation can give a picture of the DNA synthesis over the 8-16 hours pulse period, while CFSE dilution demonstrates the cell division from time point zero to the end of the assay. Thus, the two assays complement each other and both assays were used in subsequent experiments to test different aspects of blood CD11 ${ }^{+} \mathrm{Ly}_{6 \mathrm{G}}^{-}$cells' suppressive mechanisms. 

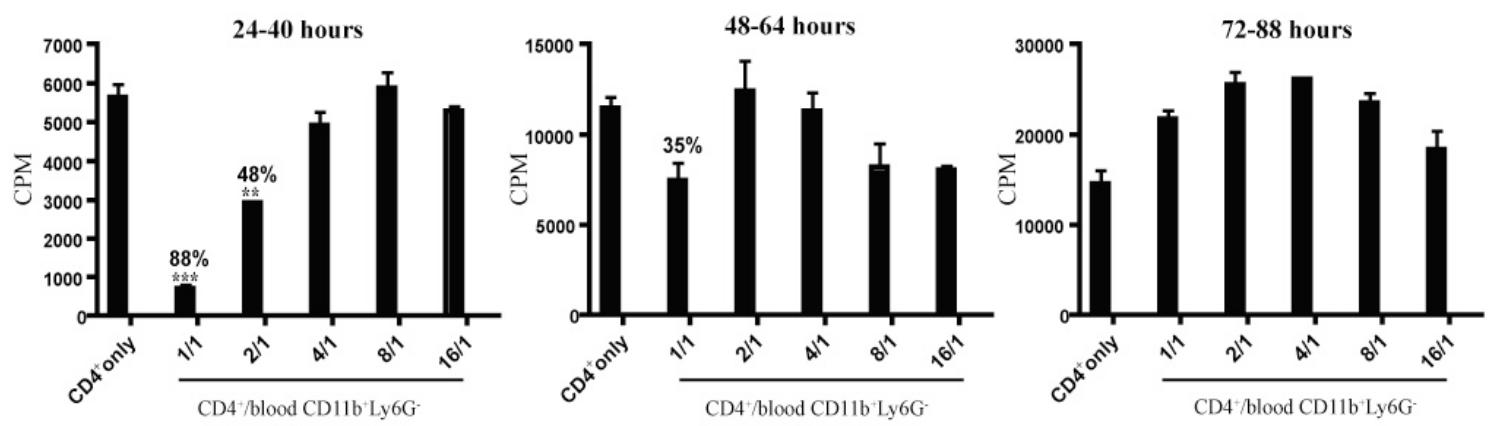

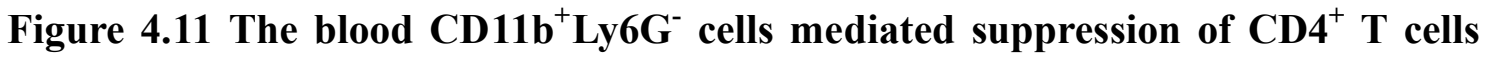
was temporary

Purified $\mathrm{CD} 4^{+}$cells $\left(50,000\right.$ cells/well) and blood $\mathrm{CD} 11 \mathrm{~b}^{+} \mathrm{Ly} 6 \mathrm{G}^{-}$cells were either cultured alone or mixed at different ratios in the presence of expander beads in a 96-well round bottom plate. The numbers indicate the period starting from the time thymidine was added to the wells to when the assays were terminated. The percentages indicate the percentages of suppression. One-way ANOVA followed by Bonferroni tests was used to analyse statistical significance, ${ }^{* * *} \mathrm{p}<0.001 ; * * \mathrm{p}<0.01$.

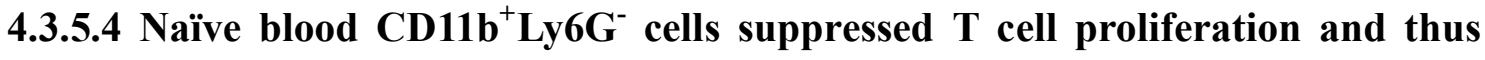 express MDSC phenotype}

To further confirm the blood $\mathrm{CD} 11 \mathrm{~b}^{+} \mathrm{Ly}_{6 \mathrm{G}}^{-}$cells express an MDSC suppressor phenotype, the purified blood $\mathrm{CD} 11 \mathrm{~b}^{+} \mathrm{Ly}_{6 \mathrm{G}}^{-}$cells were tested for their ability to suppress $\mathrm{T}$ cell proliferation in vitro in response to both antigen-non-specific and antigen-specific stimuli.

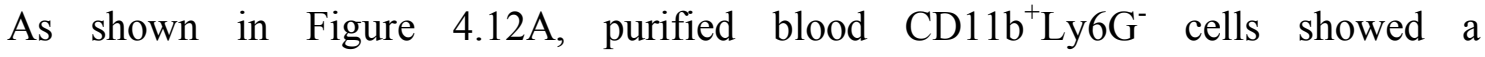
dose-dependent suppressive activity on the proliferation of splenocytes in response to the expander beads (Invitrogen, USA). Expander beads are polymer beads coated with an optimised mixture of monoclonal antibodies against the mouse CD3 and CD28 cell surface antigens, mimicking the stimulation by $\mathrm{APCs}^{198}$. The suppression of $\mathrm{T}$ cell proliferation by the blood $\mathrm{CD} 11 \mathrm{~b}^{+} \mathrm{Ly} 6 \mathrm{G}^{-}$cells was confirmed by both thymidine uptake (Figure 4.12A) and CFSE dilution of the responder cells (Figure 4.12B). The CFSE dilution assay showed that both $\mathrm{CD}^{+}$and $\mathrm{CD}^{+} \mathrm{T}$ cell proliferation was suppressed (Figure 4.12B). The blood CD11 ${ }^{+} \mathrm{Ly}_{6 \mathrm{G}^{-}}$cells were also potent suppressors of the 2D2 transgenic lymph node cell ( $\mathrm{LN}$ cell) proliferation in response to the $\mathrm{MOG}_{35-55}$ peptide (Figure 4.13). In summary, the blood $\mathrm{CD}_{11} \mathrm{~b}^{+} \mathrm{Ly}^{-} \mathrm{G}^{-}$cells appear to be potent suppressors of both antigen-non-specific and antigen-specific $\mathrm{T}$ cell responses. 
A

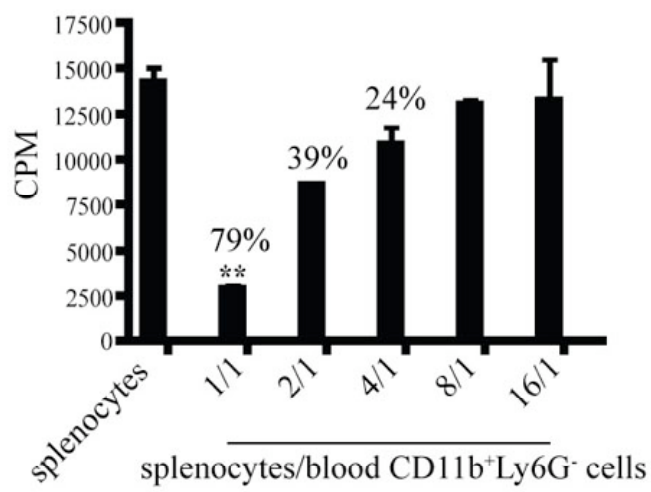

…..... splenocytes only

splenocytes+stimulation

spenocytes+blood CD11b+Ly6G- cells + stimulation

B
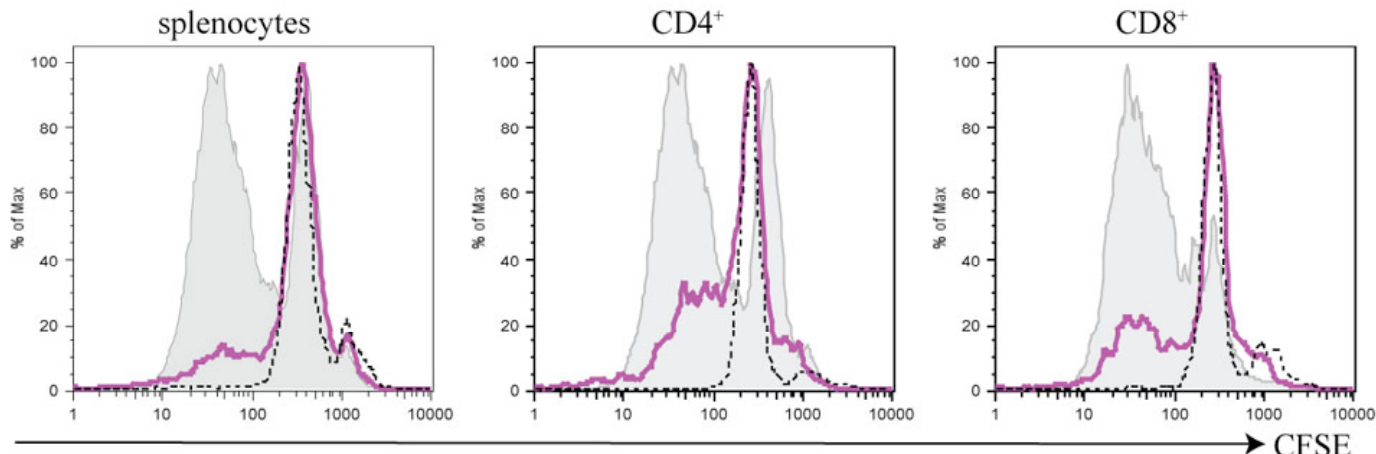

Figure 4.12 The blood $\mathrm{CD} 11 \mathrm{~b}^{+} \mathrm{Ly6G}^{-}$cells suppressed an antigen-non-specific $\mathrm{T}$ cell response

CFSE labelled 2D2 mouse splenocytes (50,000 cells/well) were either cultured alone or co-cultured with different numbers of the $\mathrm{CD} 11 \mathrm{~b}^{+} \mathrm{Ly} 6 \mathrm{G}^{-}$blood cells isolated from naïve mouse blood in a round-bottom 96-well plate. A, Cell proliferation was measured by thymidine uptake. $\left[{ }^{3} \mathrm{H}\right]$-thymidine was added to the cell culture at 24 hours, and the cells were incubated for an additional 16 hours. The numbers indicate the percentages of suppression. B, Cell proliferation was measured by CFSE dilution 72 hours after the initiation of the cell culture. $\mathrm{CD} 4^{+}$and $\mathrm{CD} 8^{+}$cell proliferation was also analysed by gating on the $\mathrm{CD} 4^{+}$ or $\mathrm{CD} 8^{+}$cell populations separately. In the cell co-culture, splenocytes and the blood $\mathrm{CD} 11 \mathrm{~b}^{+} \mathrm{Ly} 6 \mathrm{G}^{-}$were mixed at the ratio of 1:1. One-way ANOVA followed by Bonferroni tests was used to analyse statistical significance, ${ }^{* *} \mathrm{p}<0.01$. Results are representative of more than three separate experiments.

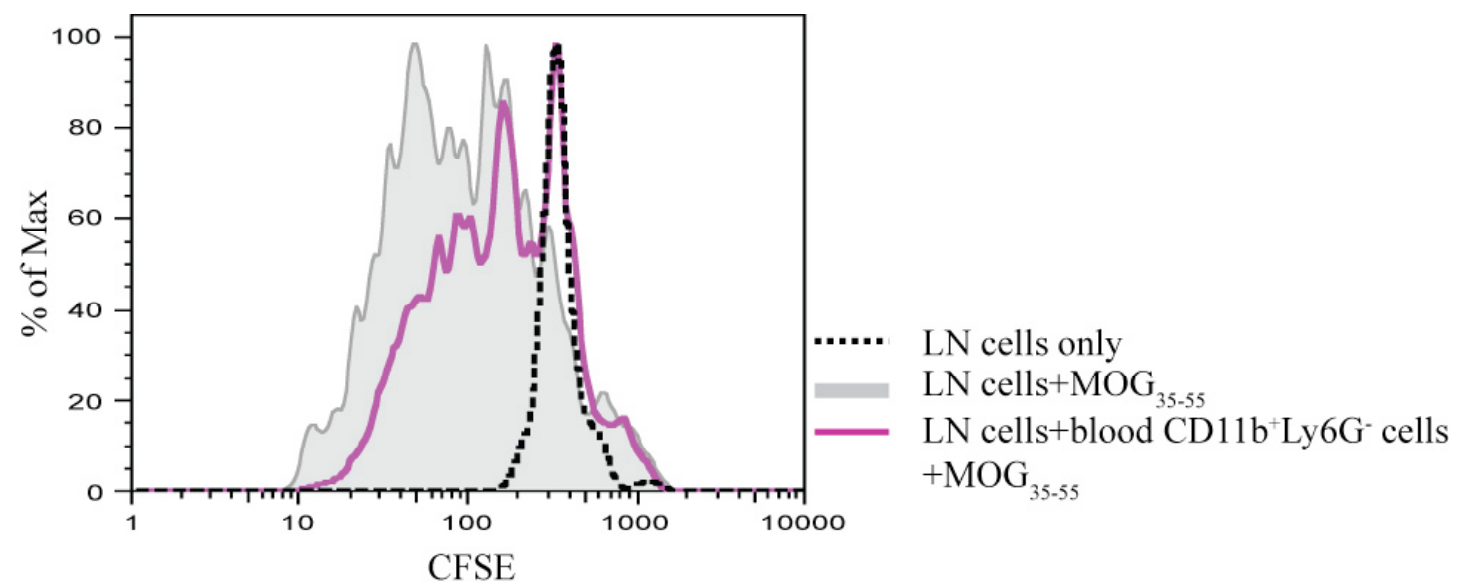

Figure 4.13 Blood CD11 ${ }^{+} \mathrm{Ly6G}^{-}$cells suppressed an antigen-specific response

CFSE labelled 2D2 transgenic LN cells $\left(50,000\right.$ cells/well) were pre-activated by $3 \mu \mathrm{g} / \mathrm{ml} \mathrm{MOG}_{35-55}$ peptide. Twenty-four hours after the incubation, purified blood $\mathrm{CD}^{1} 1 \mathrm{~b}^{+} \mathrm{Ly} 6 \mathrm{G}^{-}$cells $(50,000$ cells/well) from naïve mice were added to the culture. Cell proliferation was measured by CFSE dilution 48 hours after setting up the cell co-culture. Results are representative of more than three separate experiments. 
Bead engulfment was observed in the cell culture containing both blood CD $11 \mathrm{~b}^{+}$Ly $6 \mathrm{G}^{-}$ cells and splenocytes. In order to exclude the possibility that the observed suppression

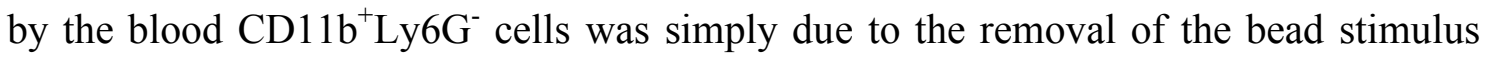
from the responder cells, the responder cells were cultured in the plate coated with anti-CD3 (Clone 145-2c11) and anti-CD28 (Clone 37.51) antibodies. As shown in Figure 4.14, the blood $\mathrm{CD} 11 \mathrm{~b}^{+} \mathrm{Ly} 6 \mathrm{G}^{-}$cells also suppressed the responder cell proliferation in this condition in a dose-dependent manner, confirming that the blood

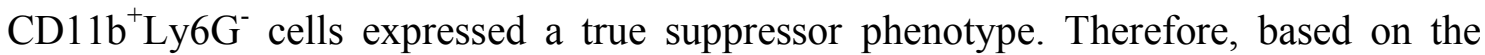
surface and functional suppressor phenotype, these blood CD11 ${ }^{+}{\text {Ly } 6 G^{-}}^{-}$cells were named blood MDSC (bMDSC).
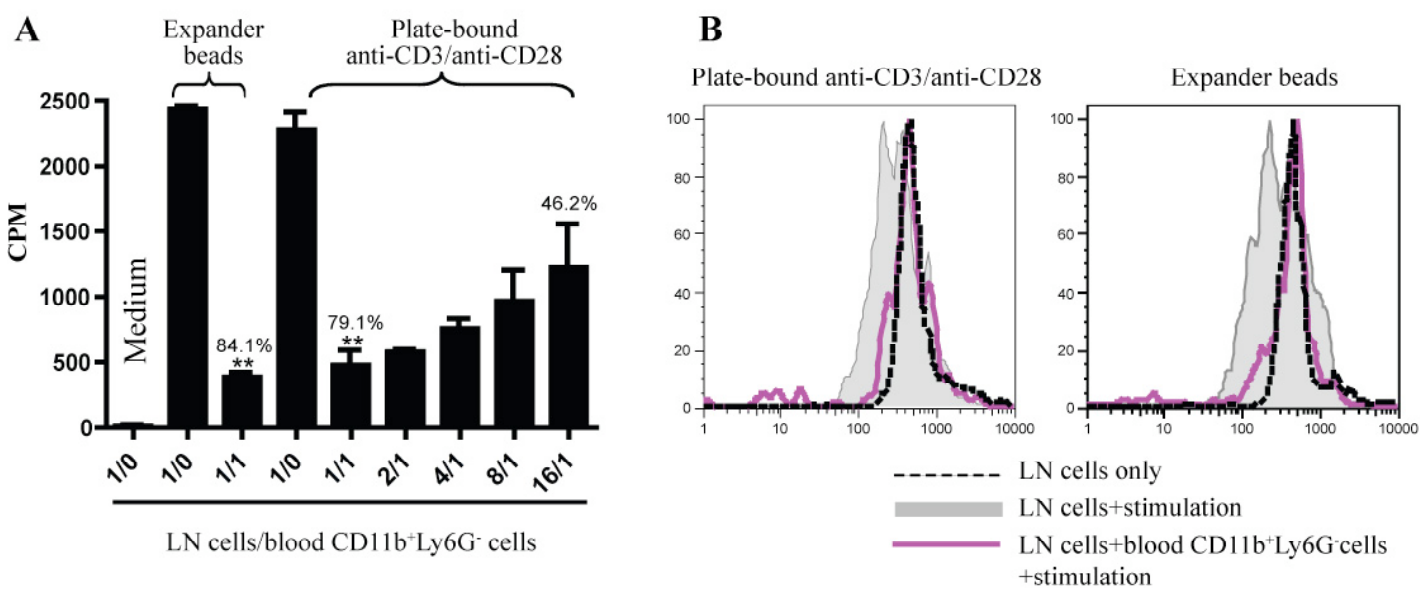

Figure 4.14 The blood $\mathrm{CD}^{11 b^{+}}{ }^{\mathrm{Ly6G}}{ }^{-}$cells suppressed the plate-bound anti-CD3 and anti-CD28 induced $T$ cell response

CFSE labelled lymph node (LN) cells $(50,000$ cells/well) were cultured with either expander beads $(25,000$ beads/well $)$ or plate bound anti-CD3 $(2 \mu \mathrm{g} / \mathrm{ml})$ and anti-CD28 $(3 \mu \mathrm{g} / \mathrm{ml})$ antibodies. Purified blood $\mathrm{CD} 11 \mathrm{~b}^{+} \mathrm{Ly} 6 \mathrm{G}^{-}$cells were added at different ratios to $\mathrm{LN}$ cells to the plates as indicated. A, $\left[{ }^{3} \mathrm{H}\right]$-thymidine was added to the plate 16 hours after initiation of the assay and the plate was harvested at 24 hours. The numbers equate to the percentage of suppression. B, CFSE dilution was measured 40 hours after the initiation of the assay. The purple line showed CFSE dilution of the LN cells incubated with the blood $\mathrm{CD} 11 \mathrm{~b}^{+} \mathrm{Ly} 6 \mathrm{G}^{-}$cells at 1:1 ratio. Cells were gated on $\mathrm{CD} 4^{+}$cells. One-way ANOVA followed by Bonferroni tests was used to analyse statistical significance, ${ }^{* *} p<0.01$. Results are representative of more than three separate experiments.

\subsubsection{Mechanisms for bMDSC-mediated suppression of $\mathrm{CD4}^{+} \mathrm{T}$ cell proliferation}

The following section provides detailed results of the investigations undertaken to understand the cellular and molecular mechanisms utilised by naïve bMDSCs to suppress $\mathrm{CD}^{+} \mathrm{T}$ cell proliferation. 


\subsubsection{1 $\mathrm{CD4}^{+}$cells were direct targets of the bMDSC-mediated suppression}

I posed the question whether the bMDSC-mediated suppression of $\mathrm{CD}^{+} \mathrm{T}$ cell proliferation acted directly on $\mathrm{CD}^{+} \mathrm{T}$ cells or indirectly by impairing APC's antigen presentation. To exclude APCs' involvement in the suppressive process, purified CD4 ${ }^{+}$ cells ( $>95 \%$ pure) were used as the responder cells and the expander beads were used as the stimuli for the suppression assay. The bMDSCs showed a robust dose-dependent suppressive activity of the purified $\mathrm{CD}^{+}$cells in vitro (Figure 4.15 ), indicating that the bMDSCs suppress $\mathrm{CD} 4^{+}$cells directly and this suppression is not dependent on APCs.

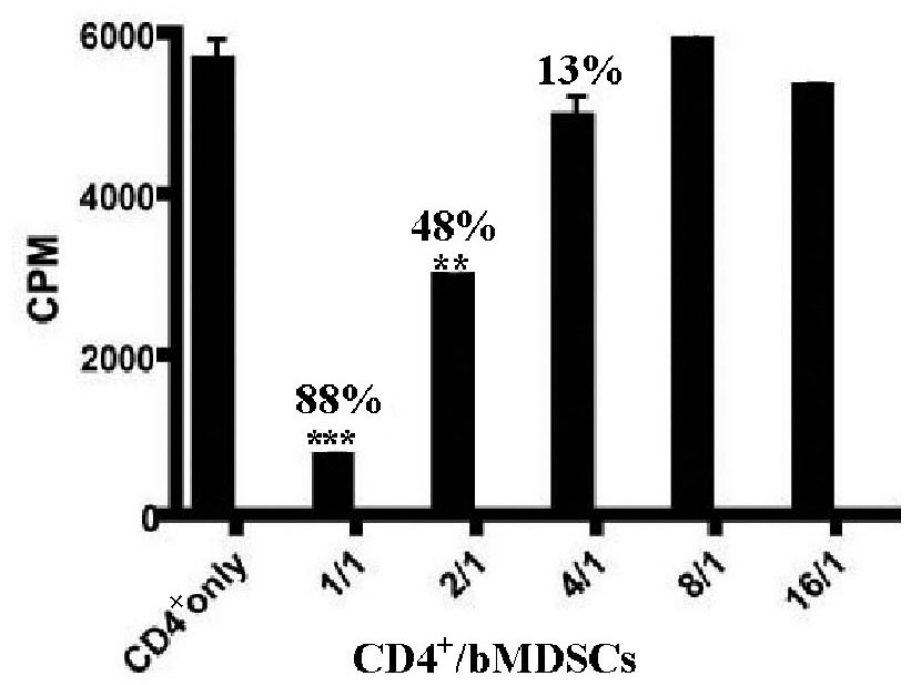

Figure 4.15 bMDSCs suppressed the proliferation of purified $\mathrm{CD4}^{+} \mathrm{T}$ cells

Purified $\mathrm{CD}^{+}$cells $(50,000$ cells/well) and bMDSCs from naïve C57BL/6J mice were either cultured alone or co-cultured at different ratios in the presence of expander beads in a 96-well round bottom plate. $\left[{ }^{3} \mathrm{H}\right]$-thymidine was added to the wells at 24 hours and incubated for additional 16 hours. The numbers indicate the percentages of suppression. One-way ANOVA followed by Bonferroni tests was used to analyse statistical significance, $* * * p<0.001 ; * * p<0.01$. Results are representative of more than three separate experiments.

\subsubsection{2 bMDSCs suppressed $\mathrm{CD}^{+} \mathrm{T}$ cell proliferation in a cell-contact dependant} manner

In order to determine whether cell contact was important for the bMDSCs' suppression of $\mathrm{T}$ cell proliferation, a transwell experiment was performed. Purified $\mathrm{CD} 4{ }^{+}$cells or unfractioned splenocytes from naïve C57BL/6J mice were incubated with the bMDSCs in the same or separate chambers of a transwell system ( $3 \mu \mathrm{m}$ pore size, Corning, USA). The suppression of $\mathrm{T}$ cell proliferation by bMDSCs was abolished when the bMDSCs and responder cells were in different chambers (Figure 4.16). This result indicates that the bMDSC-mediated suppression of $\mathrm{T}$ cell proliferation is cell contact dependent. 
To investigate the possible molecules involved in the cell-contact suppression, blocking antibodies targeting the co-stimulatory molecules CD40 (Clone 1C10), CD80 (Clone 16-10A1) and CD86 (Clone PO3.1), and the Fc $\gamma$ receptors II and III (Clone 24G2) were added to the cell co-culture. However, none of these antibodies blocked the ability of bMDSCs to suppress $\mathrm{T}$ cell proliferation (data not shown).

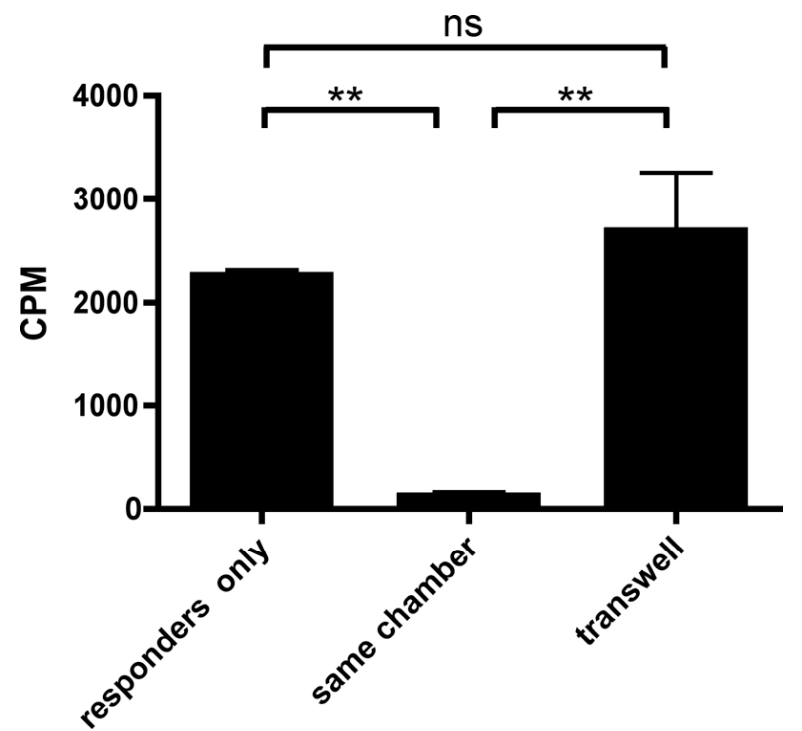

Figure 4.16 The bMDSC-mediated suppression was cell contact dependent

Unfractioned splenocytes from naïve C57BL/6J mouse were used as the responder cells $(50,000$ cells/well) and were incubated with purified bMDSCs at the ratio of 1:1 in the presence of expander beads. $\left[{ }^{3} \mathrm{H}\right]$-thymidine was added to the wells at 24 hours and incubated for an additional 16 hours. The bMDSCs and the responder cells were cultured either in the same chamber or in separate chambers in a transwell plate ( $3 \mu \mathrm{m}$ pore size) (bMDSCs were placed in the upper chamber and responder cells were in the lower chamber). One-way ANOVA followed by Bonferroni tests was used to analyse statistical significance, ${ }^{* *} \mathrm{p}<0.01$; ns, not significant. Results are representative of more than two separate experiments.

\subsubsection{NOS but not arginase 1 was involved in the bMDSCs' suppression of $T$ cell proliferation}

The majority of published evidence on the MDSCs isolated from tumour-bearing mice points to a fundamental role of nitric oxide synthase (NOS) and arginase 1 in the MDSCs' mediated suppression of T cell proliferation ${ }^{71,73}$. To investigate the possible role of NOS and arginase 1 in mediating the suppression of $\mathrm{T}$ cell proliferation by the bMDSCs, the inhibitor of NOS, $\mathrm{N}^{\mathrm{G}}$-monomethyl-L-arginine (L-NMMA) (Sigma-Aldrich, USA) and the inhibitor of arginase 1, $\mathrm{N}^{\omega}$-hydroxy-nor-L-arginine (nor-NOHA) (Calbiochem, Germany) were added to the suppression assay at $500 \mu \mathrm{M}$, the concentration previously reported to completely block NOS and arginase $1^{71,199,200}$. 
The addition of NOS inhibitor L-NMMA partially blocked the bMDSCs' suppression of $\mathrm{T}$ cell proliferation (Figure 4.17), indicating a nitric oxide (NO)-dependent mechanism. The failure of L-NMMA to completely reverse the suppression indicated the existence of an additional NO-independent suppressive mechanism. In contrast, arginase 1 inhibitor nor-NOHA failed to reduce the bMDSCs' suppression. Thus, arginase 1 activity did not contribute to the bMDSCs' suppression of $\mathrm{T}$ cell proliferation. In addition, adding nor-NOHA to the cell co-culture containing L-NMMA did not further reduce the bMDSCs' suppressive activity indicating that there was no synergistic effect of NOS and arginase 1 in this bMDSC-dependent suppression. In conclusion, NOS but not arginase 1 plays a role in bMDSC-mediated suppression of $\mathrm{T}$ cell proliferation.

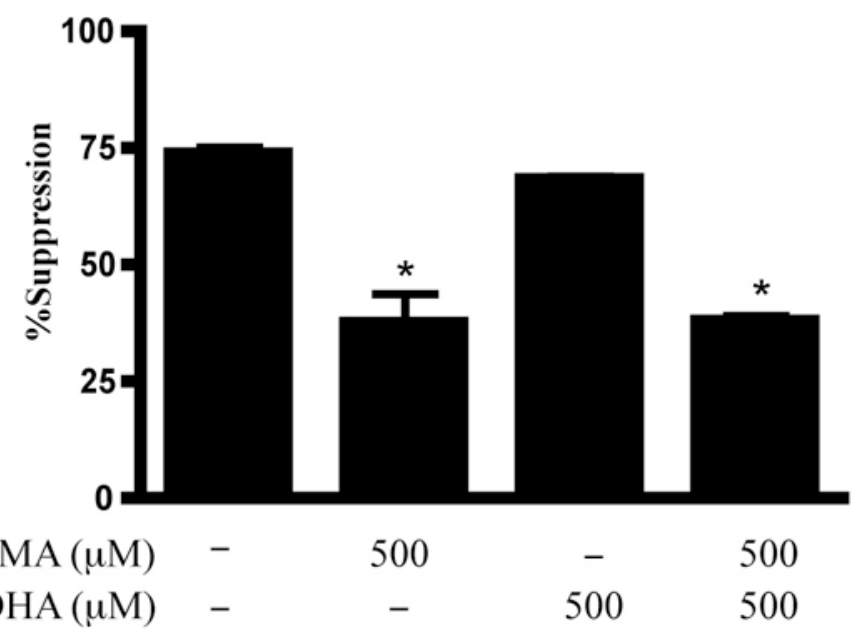

Figure 4.17 The bMDSCs' suppression of $T$ cell proliferation was mediated by NOS but not arginase 1

C57BL/6J splenocytes and purified bMDSCs were co-cultured at 1:1 ratio with expander beads. L-NMMA or nor-NOHA were supplemented to the cell culture as indicated. $\left[{ }^{3} \mathrm{H}\right]$-thymidine was added to the plate 24 hours after the initiation of the assay and the plate was harvested after another 16 hours. One-way ANOVA followed by Bonferroni tests was used to analyse statistical significance, ${ }^{*} \mathrm{p}<0.05$.

\subsubsection{The role of IFN- $\gamma$ in the bMDSC-mediated suppression of $T$ cell proliferation}

A number of studies have shown that IFN- $\gamma$ plays an important role in the MDSC-mediated suppression of $\mathrm{T}$ cell responses ${ }^{71,169,201}$. It is therefore possible that IFN- $\gamma$ also plays a role in naïve bMDSC-dependent suppression of $\mathrm{T}$ cell proliferation. To determine whether IFN- $\gamma$ is required for bMDSCs to suppress $\mathrm{T}$ cell proliferation, and if it is required, the cellular source of IFN- $\gamma$, IFN- $\gamma$ deficient (IFN $\gamma-/-$ ) cells and the IFN- $\gamma$ neutralising antibody (Clone An-18) were tested in the suppression assay. As 
shown in Figure 4.18, the bMDSCs derived from IFN $\gamma-/-$ mice suppressed both wild type and IFN $\gamma$-/- responder cells at levels comparable to that of the wild type bMDSCs. Importantly, the IFN $\gamma-/-$ bMDSCs strongly suppressed the IFN $\gamma-/-$ responder cells. IFN- $\gamma$ was absent in this condition, indicating that bMDSCs suppress $T$ cells in an IFN- $\gamma$ independent manner.

Although the difference in suppression between IFN $\gamma$-/- and wild type bMDSCs was not significant, there was a trend towards reduced suppression in the IFN $\gamma-/-$ MDSCs. The observed difference in suppression may be due to the anti-proliferative effects of IFN- $\gamma$ in the co-culture ${ }^{187,202-205}$, or a result of the inherent differences in two distinctly different populations i.e. the bMDSCs derived from a wild type environment versus bMDSCs derived from an IFN- $\gamma$ devoid environment. To understand whether this marginal difference was due to IFN- $\gamma$ in the cell co-culture, IFN- $\gamma$ neutralising antibody was added to block IFN- $\gamma$-mediated responses. The addition of the IFN- $\gamma$ antibody slightly reversed the suppression by the bMDSCs (Figure 4.18B). Again, the difference in suppression between the cell cultures was not statistically significant, indicating that although IFN- $\gamma$ maybe involved to a small degree, IFN- $\gamma$ is not the key mediator in the suppressive activity of naïve bMDSCs on T cell proliferation.

A

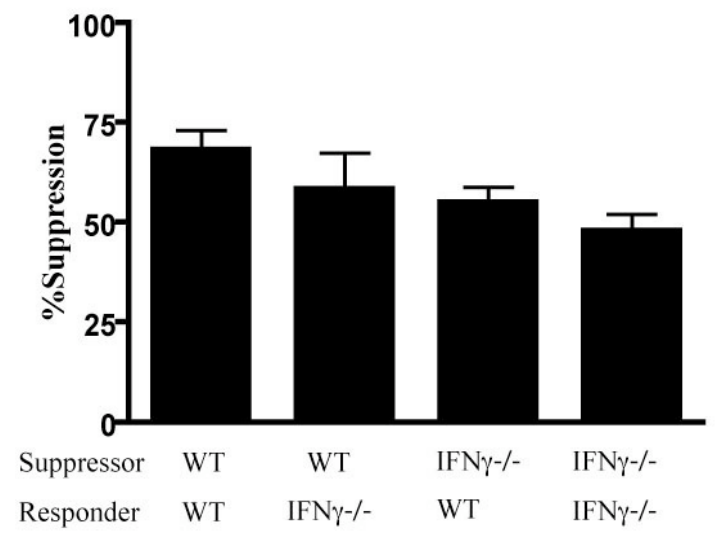

B

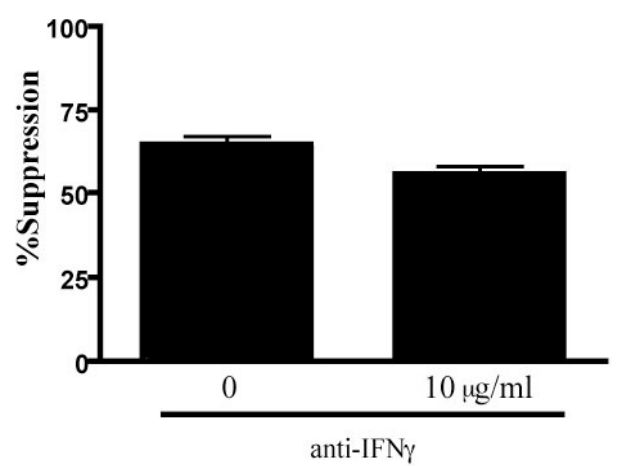

Figure 4.18 The involvement of IFN- $\gamma$ in the bMDSCs' suppression of $T$ cell proliferation

A, Splenocytes derived from either C57BL/6J or IFN-/- mice were incubated with purified bMDSCs from either $\mathrm{C} 57 \mathrm{BL} / 6 \mathrm{~J}$ or IFN $\gamma-/-$ mice at 1:1 ratio in the presence of expander beads. B, C57BL/6J splenocytes were incubated with the bMDSCs purified from C57BL/6J mice at 1:1 ratio in the presence of neutralising IFN- $\gamma$ antibody at $10 \mu \mathrm{g} / \mathrm{ml}$. \%suppression $=(1$-proliferation with the bMDSCs in the presence of anti-IFN- $\gamma /$ proliferation without the bMDSCs in the presence of anti-IFN- $\gamma$ ) $\times 100 \%$. $\left[{ }^{3} \mathrm{H}\right]$-thymidine was added to the plate 24 hours after the initiation of the assay and the plate was harvested after another 16 hours. WT, wild type. 


\subsubsection{5 bMDSCs' suppression of $T$ cell proliferation was not dependent on IDO}

Indoleamine-2,3-dioxygenase (IDO) is an enzyme that degrades the essential amino acid L-tryptophan ${ }^{186,206-210}$, and this degradation halts growth of $\mathrm{T}$ cells ${ }^{208}$. IDO has been reported to be induced in macrophages ${ }^{208}$ and DCs ${ }^{209,210}$ after exposure to IFN- $\gamma$. Since the observed suppression by the bMDSCs was partially mediated by IFN- $\gamma$, it was possible that IDO played a role in the bMDSC-mediated suppression. Therefore, the bMDSCs from IDO deficient mice (IDO-/-) were tested for their suppressive effect. The bMDSCs purified from wild type and IDO-/- mice exhibited comparable suppressive activity of $\mathrm{T}$ cell proliferation and the IDO-/- bMDSCs strongly suppressed the IDO-/responder cells (Figure 4.19). Therefore, the bMDSC-mediated suppression was not IDO dependent.

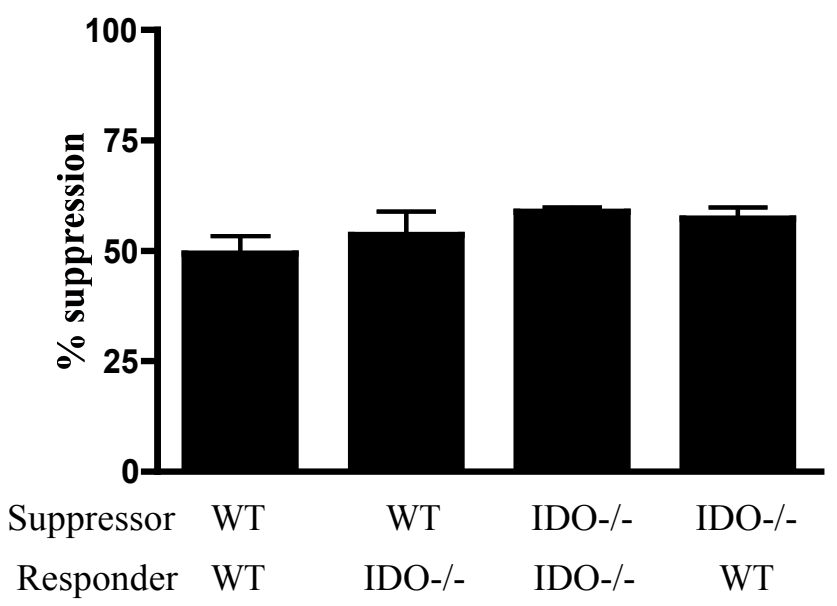

Figure 4.19 IDO was not involved in the bMDSC-mediated suppression of $T$ cell proliferation

Splenocytes derived from either C57BL/6J or IDO-/- mice were incubated with the expander beads in the presence of purified bMDSCs from either $\mathrm{C} 57 \mathrm{BL} / 6 \mathrm{~J}$ or IDO-/- mice at $1: 1$ ratio. $\left[{ }^{3} \mathrm{H}\right]$-thymidine was added to the plate 24 hours after the initiation of the assay and the plate was harvested after another 16 hours. Results are representative of more than two separate experiments. WT, wild type.

\subsubsection{The bMDSCs did not enhance responder cell apoptosis}

To determine whether the bMDSC-mediated suppression of $\mathrm{T}$ cell proliferation could be due to the induction of apoptosis on the responder cells, bMDSCs were cultured with CFSE labelled splenocytes for 48 hours and the apoptotic marker Annexin V was analysed on the splenocytes at 4, 8, 16, 32 and 48 hours. As shown in Figure 4.20, the addition of bMDSCs did not tend to increase the responder cell apoptosis. In contrast, in the presence of bMDSCs, the splenocytes had a reduced percentage of Annexin $\mathrm{V}^{+}$cells 
e.g. at 48 hours (Figure 4.20). The higher percentage of Annexin $\mathrm{V}^{+}$cells in the cell culture that did not contain bMDSCs can be explained by over-activation related apoptosis of the responder $\mathrm{T}$ cells ${ }^{211}$. Because the splenocytes cultured with the bMDSCs did not proliferate as much, over-activation related apoptosis might not have occurred. In conclusion, bMDSCs did not enhance apoptosis in the responder cells.

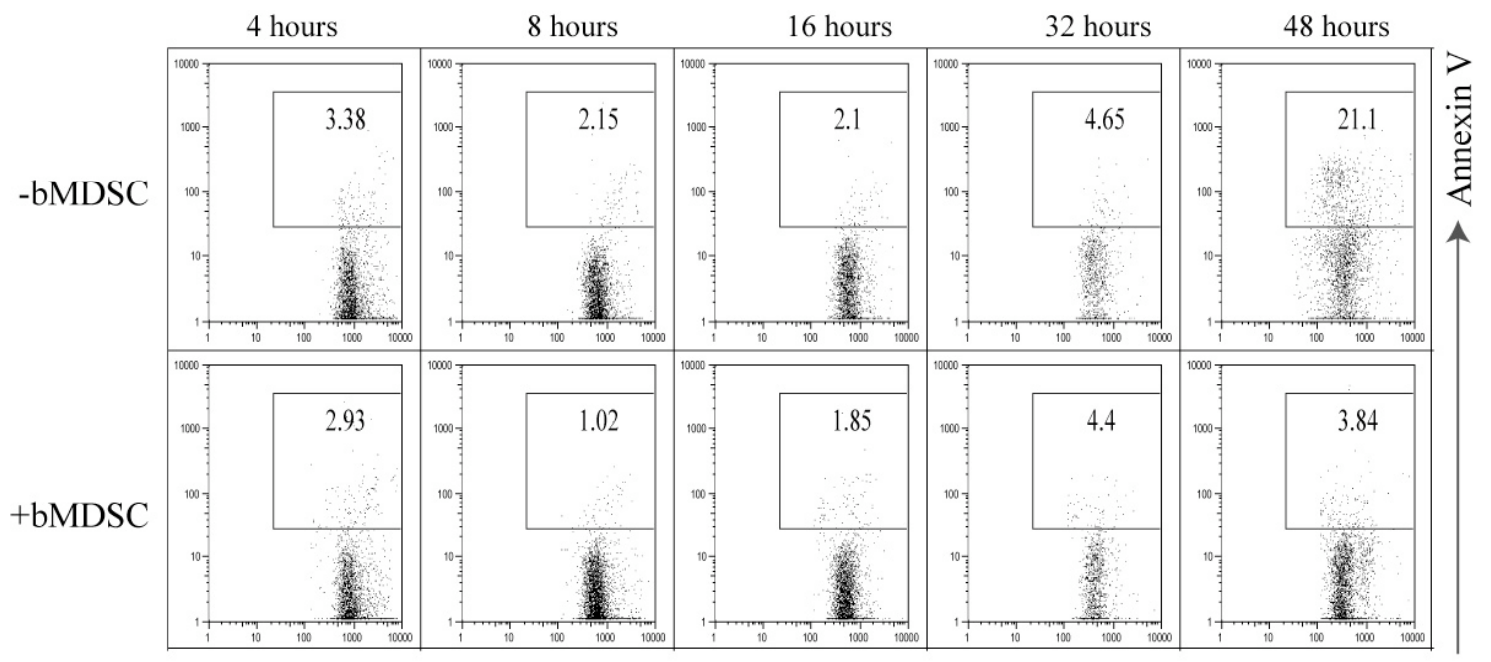

Figure 4.20 The addition of the bMDSCs did not enhance the splenocyte apoptosis Splenocytes derived from C57BL/6J mice (50,000 cells/well) were labelled with CFSE and incubated with expander beads in the presence of purified bMDSCs at 1:1 ratio. At 4,8,16,32 and 48 hours, the cells were stained with Annexin V and analysed by flow cytometry. Cells were gated on the CFSE ${ }^{+}$responder cells. The numbers indicate the percentages of Annexin $\mathrm{V}^{+}$cells of the $\mathrm{CFSE}^{+}$cells.

\subsubsection{The bMDSC-mediated suppression of $T$ cell proliferation was not dependent on Tregs}

It has been reported that the MDSCs derived from tumour-bearing mice could induce the expansion of Foxp3 $3^{+}$Tregs $^{73}$. It was therefore possible that bMDSCs-mediated suppression of $\mathrm{T}$ cell proliferation was an indirect result of the expansion of Tregs from responder cell population. To assess whether Tregs were important in the bMDSCs' suppressive activity, Foxp3-GFP KI male mice were injected with anti-CD25 antibody (Clone PC61) two days prior to the isolation of responder cells. PC61 antibody has been reported to partially neutralise Tregs and functionally inactivate the remaining Tregs in vivo ${ }^{162}$. The neutralising of Tregs in PC61-treated mice was confirmed by analysing surface CD25 (Clone 7D4) and Foxp3-GFP expression on $\mathrm{CD}^{+}$cells. As shown in Figure 4.21A, PC61 administration resulted in the neutralisation of CD25 $5^{+}$cells (from $6-7 \%$ to $1-2 \%$ ), and a significant decrease in Foxp $3^{+}$cells (from $9-10 \%$ to $4-6 \%$ ). 
$\mathrm{CD}^{+}$cells were purified from either PC61 treated mice as described above or naïve mice and co-incubated with bMDSCs derived from naïve mice. As shown in Figure $4.21 \mathrm{~B}$, the $\mathrm{CD}^{+}$cells from PC61 treated mice were equally suppressed by bMDSCs as the $\mathrm{CD}^{+}$cells derived from naïve mice, indicating that bMDSCs do not depend on nTregs to suppress $\mathrm{CD} 4^{+} \mathrm{T}$ cell proliferation.
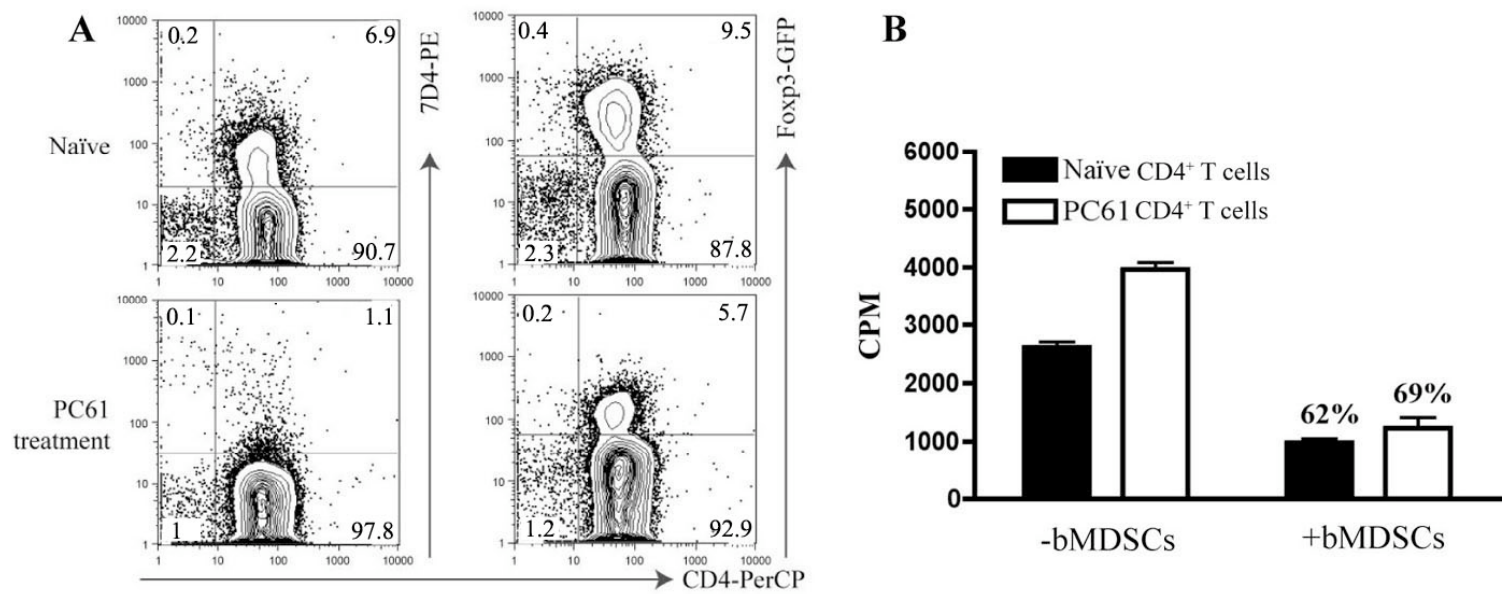

Figure 4.21 bMDSCs-mediated suppression of $\mathrm{CD}^{+} \mathrm{T}$ cell proliferation did not depend on Tregs

Foxp3-GFP KI male mice were injected i.p. once with anti-CD25 antibody (Clone PC61) at a dose of $200 \mu \mathrm{g}$ or left untreated as the experimental controls. $\mathrm{CD}^{+}$cells were purified from the lymph nodes two days after the injections. The purified $\mathrm{CD}^{+}$cells were analysed by flow cytometry for $\mathrm{CD} 4$, Foxp3 and $\mathrm{CD} 25$ expression. A, Staining of the purified $\mathrm{CD}^{+}$cells analysed for $\mathrm{CD} 4, \mathrm{CD} 25$ (Clone 7D4) and Foxp3-GFP expression. Numbers indicate percent of total cells in each quadrant. B, The purified $\mathrm{CD}^{+}$ cells were co-cultured with bMDSCs at a ratio of 1:1 in the presence of expander beads for 24 hours. $\left[{ }^{3} \mathrm{H}\right]$-thymidine was added to the cell culture and incubated for a further 16 hours. The numbers indicate the percentages of suppression by bMDSCs. Results are representative of more than two separate experiments.

\subsubsection{Binding of DM with bMDSCs in vivo enhanced the bMDSCs' suppression of}

\section{T cell proliferation in vitro}

After confirming bMDSCs' suppressive phenotype, I next investigated whether the uptake of DM by bMDSCs altered the cells' suppressive activity. Three hours after i.v. injection of DM-Alexa 488, bMDSCs were isolated from the injected mice for an in vitro suppression assay. The uptake of DM by bMDSCs was confirmed by flow cytometric analysis (Figure 4.3). As shown in Figure 4.22A, compared with the naïve bMDSCs, the $\mathrm{DM}^{+}$bMDSCs exhibited enhanced suppressive activity in vitro and retained this suppressive activity for longer (Figure 4.22B). The enhanced suppressor function of the bMDSCs loaded with DM highlights the potential for DM to be utilised as an immunosuppressive therapy for autoimmune diseases by targeted manipulation of bMDSCs in vivo. 
A

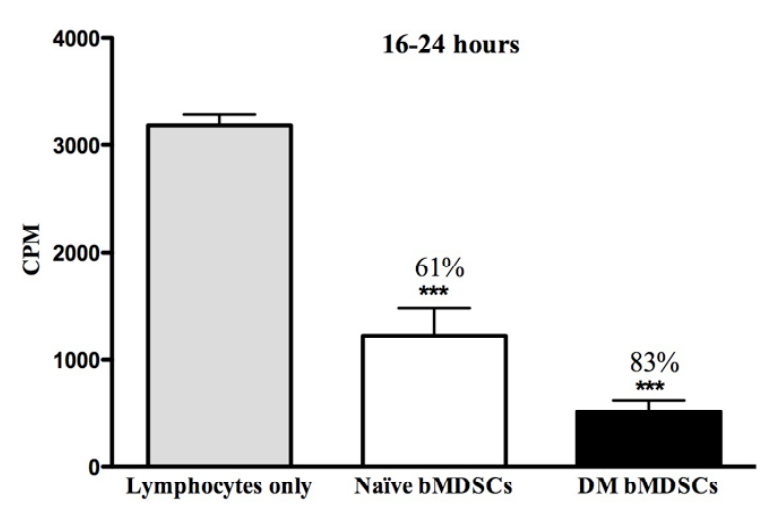

B

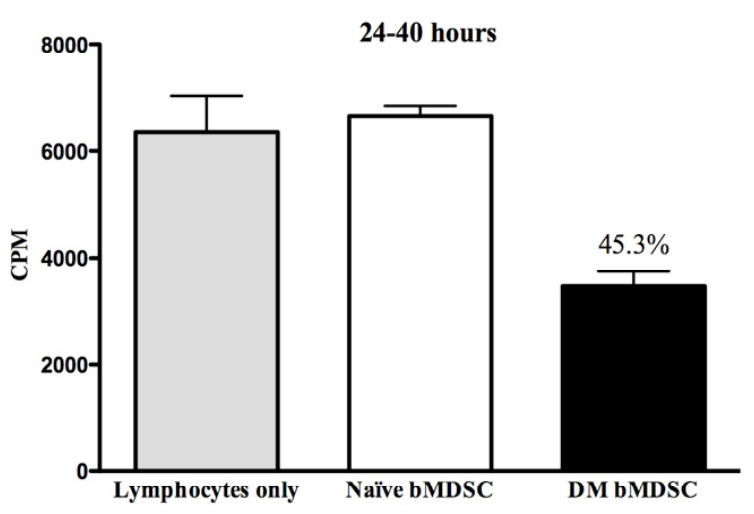

Figure 4.22 The binding of DM with bMDSCs in vivo enhanced the bMDSC-mediated suppression of $T$ cell proliferation in vitro

DM-Alexa $488(50 \mu \mathrm{g})$ was injected into C57BL/6J mice i.v. Three hours after the injection, the binding of DM to bMDSCs was verified by flow cytometry analysis on the blood cells. Purified bMDSCs were co-cultured with the splenocytes derived from naïve $\mathrm{C} 57 \mathrm{BL} / 6 \mathrm{~J}$ mice at $1: 1$ ratio in the presence of plate-bound anti-CD3 $(2 \mu \mathrm{g} / \mathrm{ml})$ and anti-CD28 $(3 \mu \mathrm{g} / \mathrm{ml})$ antibodies in a 96-well plate. The bMDSCs purified from naïve mice were used as the experimental control. The suppression of $\mathrm{T}$ cell proliferation was measured at A, 16-24 hours; B, 24-40 hours after the initiation of the assay by thymidine incorporation. The numbers on the graph indicate \%suppression. One-way ANOVA followed by Bonferroni tests was used to analyse statistical significance, $* * * \mathrm{p}<0.0001$. Results are representative of more than two separate experiments.

\subsubsection{DM did not bind to bMDSCs in vitro}

As demonstrated in the previous section, the binding of DM with bMDSCs in vivo strongly enhanced the bMDSCs' ability to suppress T cell proliferation. Given this and that experimental procedures are easier in an in vitro set-up, I attempted to load bMDSCs with DM in vitro. Surprisingly, isolated bMDSCs did not bind to the bMDSCs in vitro regardless of the culture environment (Table 4.2). The inability of the isolated bMDSCs to bind to DM points towards the involvement of a yet unknown in vivo mechanism for DM uptake by bMDSCs. 
Table 4.2 Methods utilised to investigate binding of DM-Alexa 488 to bMDSCs in vitro

\begin{tabular}{|c|c|}
\hline Cells types isolated & 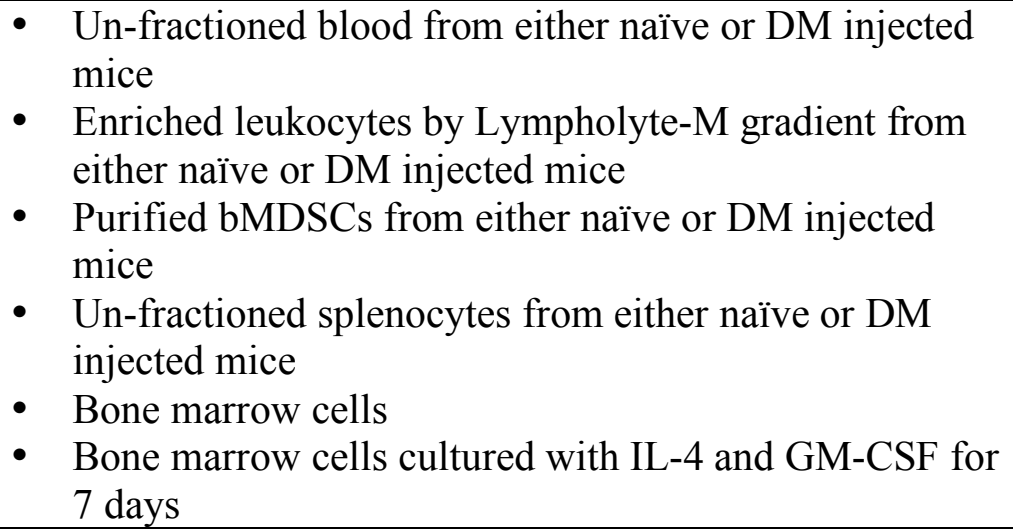 \\
\hline Anti-aggregation methods & $\begin{array}{ll}- & \text { Heparin } \\
\text { - } & \text { Alsevers solution } \\
\end{array}$ \\
\hline Staining buffer & $\begin{array}{ll}\text { - } & \text { cIMDM } \\
\text { - } & \text { IMDM } \\
\text { - } & \text { IMDM containing mouse blood serum } \\
\text { - } & \text { IMDM containing the blood serum extracted from DM } \\
& \text { injected mice } \\
\text { - } & \text { Naïve mouse blood serum } \\
\text { - } & \text { Mouse blood serum extracted from DM injected mice } \\
\text { - } & \text { HBSS } \\
\text { - } & \text { PBS } \\
\text { - } & \text { FACS buffer } \\
\text { - } & \text { PBS containing } 0.2 \mathrm{mM} \mathrm{ZnCl}_{2} \\
\text { - } & \text { PBS containing } 0.2,1 \text { or } 5 \mathrm{mM} \mathrm{MgSO}_{4} \\
\text { - } & \text { PBS containing } 0.02,0.1 \text { or } 0.5 \mathrm{mM} \mathrm{MnCl} \mathrm{mM}_{2} \\
\text { - } & \text { PBS containing } 0.2 \mathrm{mM} \mathrm{MgSO}_{4} \mathrm{MgS}_{4} \text { and } 0.02 \mathrm{mM} \mathrm{M} \mathrm{MnCl}_{2} \\
\text { - } & \text { PBS containing } 5 \mathrm{mM} \mathrm{MgSO}_{4} \text { and } 0.5 \mathrm{mM} \mathrm{MnCl}_{2} \\
\end{array}$ \\
\hline Stimulation & $\begin{array}{ll}- & \text { LPS } \\
\text { - } & \text { Pam3Cys } \\
\text { - } & \text { PMA/ionomycin } \\
\text { - } & \text { Anti-CD3 antibody (Clone } 145-2 \mathrm{c} 11) \\
\end{array}$ \\
\hline
\end{tabular}

\subsection{Discussion}

The overall aim of the experiments presented in this chapter was to investigate whether the blood cells that DM binds to in vivo are able to suppress immune responses and if so, to investigate the cellular and molecular mechanisms these cells utilised in this process. In this chapter, I found that the $\mathrm{DM}^{+}$blood cells are mononuclear in

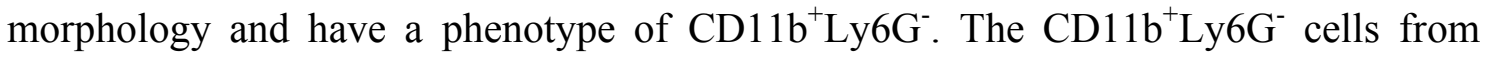
both naïve mice and DM-injected mice expressed the common surface markers for MDSCs such as CD11b, F4/80 and Gr-1 (Table 4.1) and potently suppressed T cell 
proliferation in vitro. Based on their myeloid phenotype and highly suppressive nature, I have classified these cells as "bMDSCs".

In recent years, the $\mathrm{Gr}-1^{+} \mathrm{CD} 11 \mathrm{~b}^{+}$MDSCs have been extensively studied in tumour models and are considered critical in assisting tumours to escape immune recognition ${ }^{45,75,76}$. In tumour models, MDSCs aberrantly expand during the process of tumour growth ${ }^{71}$ and tumour environment greatly influences MDSCs' characteristics $^{71,212}$. For example, the splenic MDSCs isolated from tumour-bearing mice exhibit a marked reduction in interferon regulatory factor-8 (IRF) expression compared to the $\mathrm{Gr}-1^{+} \mathrm{CD} 11 \mathrm{~b}^{+}$splenocytes derived from naïve mice. IRF-8 plays an integral role in the regulation of cell death and the reduction of IRF-8 leads to increased MDSC survival ${ }^{213}$. Although it is known that the $\mathrm{Gr}-1^{+} \mathrm{CD} 11 \mathrm{~b}^{+}$MDSCs from tumour-bearing mice are potent suppressors of immune responses, and the Gr- $1^{+} \mathrm{CD} 11 \mathrm{~b}^{+}$cells also exist in naïve mice ${ }^{71,169}$, there had been no evidence that freshly isolated $\mathrm{Gr}-\mathrm{1}^{+} \mathrm{CD} 11 \mathrm{~b}^{+}$cells from the naïve mouse blood were able to suppress a $\mathrm{T}$ cell response before this study.

The bMDSCs described in this thesis are different from the $\mathrm{Gr}-1^{+} \mathrm{CD} 11 \mathrm{~b}^{+}$splenic MDSCs accumulated in tumour models in that bMDSCs were isolated from the blood of naïve mice. Although cells sharing the same cell surface markers (CD11b ${ }^{+}$Ly6G ${ }^{-}$ $\mathrm{F} 4 / 80^{+}$) were also present in the spleens of naïve mice, and these cells could also bind to

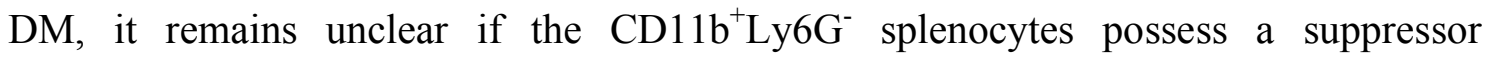
phenotype. In the literature, results of measuring the in vitro suppressive activity of the Gr- $1^{+} \mathrm{CD} 11 \mathrm{~b}^{+}$splenocytes from tumour-free mice are not consistent. Most reports indicate that the Gr- $1^{+} \mathrm{CD} 11 \mathrm{~b}^{+}$splenocytes are not suppressive $\mathrm{e}^{71,74,84-86}$, except that one paper shows that these cells are able to suppress $\mathrm{CD}^{+} \mathrm{T}$ cell responses ${ }^{87}$. However, the Gr- $1^{+} \mathrm{CD} 11 \mathrm{~b}^{+}$splenocytes used in that study express very high level of Gr-1, and are likely to be $\mathrm{Ly} 6 \mathrm{G}^{+}$neutrophilic cells. In contrast, this thesis focused on the $\mathrm{Ly}^{-} \mathrm{G}^{-}$ mononuclear bMDSCs freshly isolated from the blood of naïve mice. The existence of the bMDSC population in a natural disease-free condition suggests that bMDSCs are a suppressive mechanism that has evolved to maintain immune homeostasis and limit uncontrolled immune responses. 
The bMDSCs described in this thesis are also different from the $\mathrm{Gr}-1^{+} \mathrm{CD} 11 \mathrm{~b}^{+}$bone marrow suppressor cells reported in other studies ${ }^{214-216}$. It is shown that after the Gr- $1^{+} \mathrm{CD} 11 \mathrm{~b}^{+}$and other non-adherent bone marrow cells are cultured with different cytokines such as IFN- $\gamma$, GM-CSF and IL-3, these cells are able to suppress T cell responses $^{214-216}$. The plasticity of myeloid cells has been well documented ${ }^{217}$ and thus, it is likely that the in vitro stimulation by the above cytokines results in the differentiation of these myeloid cells and endows them a suppressive capability. In contrast, bMDSCs are derived from the blood and are able to suppress $\mathrm{T}$ cell proliferation without any additional stimulation. Therefore, bMDSCs are different from the reported bone marrow suppressor cells.

Two reported suppressor cell populations have many features similar to the bMDSCs described in this thesis ${ }^{71,169}$. In the first study, Zhu et al reported a population of inflammatory monocytes (IMC) in EAE mouse spleens that exhibit many features in common with the bMDSCs described in this chapter, e.g. monocytic morphology ${ }^{169}$. However, IMCs are different from bMDSCs in their functional phenotype. Zhu et al observed a strong induced apoptosis in proliferating $\mathrm{CD}^{+} \mathrm{T}$ cells co-cultured with IMCs, and the IMC derived from IFN $\gamma-/$ - mice could not suppress T cell proliferation. In contrast, bMDSCs did not induce $\mathrm{T}$ cell apoptosis and the IFN $\gamma$-/- bMDSCs were almost equally suppressive as the wild type ones. In the second study, the mononuclear MDSCs (MO-MDSCs) described by Movahedi et $a l^{71}$ in tumours also suppress $\mathrm{T}$ cell responses in a NOS-dependent manner. However, MO-MDSCs vary in their abilities to suppress $\mathrm{T}$ cell activation depending on which tumours they are derived from, indicating that tumour microenvironments have changed these cells' suppressive function. It remains unclear if these MO-MDSCs and the bMDSCs described in this chapter arise from the same progenitors. It is noteworthy that bMDSCs are derived from naïve mouse blood and MO-MDSCs are from the spleens of tumour-bearing mice.

It is of interest that the in vivo "loading" of DM to bMDSCs enhanced the cells' suppressive activity, in aspects of both potency and duration. Since naïve bMDSCs used NO to suppress $\mathrm{T}$ cell proliferation, it was possible that the observed increase of suppression was associated with an enhanced NO secretion by bMDSCs. However, no significant difference in NO level was observed in the supernatant of the cell cultures 
containing DM-loaded or empty bMDSCs (data not shown). It was likely that the observed enhancement of bMDSCs' suppression by DM loading was caused by other mechanisms rather than the increased NO secretion. Further studies need to be done to understand the mechanisms DM utilised to enhance bMDSCs' suppression. This enhanced suppression reveals DM's inhibitory ability and highlights its potential to be utilised as an immunosuppressive agent targeting bMDSCs in vivo.

Although great effort has been made, the binding of DM and bMDSCs has not been achieved in vitro. Further studies are required to understand possible explanations for the failure of the binding between DM and bMDSCs in vitro.

The findings from this chapter underscore a previously unknown role of bMDSCs in limiting $\mathrm{CD}^{+} \mathrm{T}$ cell responses and highlight the potential for developing an immunosuppressive therapy through the manipulation of bMDSCs. The description of bMDSCs in naïve mice provides a mechanistic insight into the body's immunological tolerance. Moreover, $\mathrm{CD}^{+} \mathrm{T}$ cells are the pathogenic cells in EAE model ${ }^{111,13,137,144}$, and the understanding of bMDSCs' suppression of $\mathrm{CD}^{+}$cell proliferation may be important for developing potential immunotherapies for EAE, and hence MS. Nevertheless, the finding that the binding of DM to bMDSCs greatly enhanced these cells' suppressive activity identifies the potential for DM to be utilised as an immunosuppressive agent targeting bMDSCs in vivo.

\subsection{Conclusions}

In this chapter, I discovered and defined a specific suppressor cell population residing in the blood and named them "bMDSCs". These cells are potent suppressors of T cell responses in vitro. The existence of this bMDSC population in naïve mice suggests that these cells are part of the body's regulatory mechanism and may have evolved to maintain immune homeostasis and limit immune responses. In addition, the binding of DM to bMDSCs strongly enhances these cells' suppression of T cell proliferation. This enhanced suppression caused by the DM-bMDSCs binding highlights the potential for using DM as an immunosuppressive agent targeting bMDSCs in vivo. The therapeutic potential of DM was investigated in Chapter 5 using the mouse EAE model. 


\section{Chapter 5:}

\section{DM-MOG $_{35-55}$ Suppresses EAE in a MOG $_{35-55}$ Specific Manner}




\subsection{Introduction}

Theoretically, autoimmune diseases such as MS could be inhibited using therapies that specifically suppress the activation of auto-reactive responses. However, there has been limited success in developing highly specific treatments to suppress autoimmune responses without impairing the body's global immunity. In Chapter 3, I showed that adding SM-MOG $35-55$ to the EAE inducing emulsion brought about a slight suppression of the disease. However, it was unclear whether SM-MOG $35-55$ mediated EAE suppression was $\mathrm{MOG}_{35-55}$ specific. Additionally, SM-MOG $35-55$ did not activate or expand Tregs as hypothesised, but instead, SM bound to a suppressive subset of cells, which were described and termed as bMDSCs in Chapter 4. This raised the possibility that the bMDSCs played a role in $\mathrm{SM}_{-} \mathrm{MOG}_{35-55}$ mediated EAE suppression.

In Chapter 4, I also showed that DM possessed an enhanced binding capability to bMDSCs compared to SM. Surprisingly, the in vivo binding of DM to bMDSCs improved the bMDSCs' suppression of T cell proliferation in vitro. Given the enhanced binding of DM to bMDSCs compared to SM and the ability of DM to augment bMDSC-mediated $\mathrm{T}$ cell suppression, $\underline{\mathrm{I} \text { hypothesised that } \mathrm{DM}-\mathrm{MOG}_{35-55}} \underline{\text { could suppress }}$ EAE, and that bMDSCs play a key role in DM-MOG $35-55$ mediated suppression of EAE.

\subsection{Aims}

The experiments described in this chapter aimed to test the above hypothesis and develop a successful immunotherapy for EAE as a potential treatment for MS.

The specific aims were as follows:

- To determine if the conjugate $\mathrm{DM}-\mathrm{MOG}_{35-55}$ could protect mice from developing EAE

- To investigate if the $\mathrm{MOG}_{35-55}$ peptide conjugated to DM was necessary for $\mathrm{DM}_{-} \mathrm{MOG}_{35-55}$ mediated EAE suppression

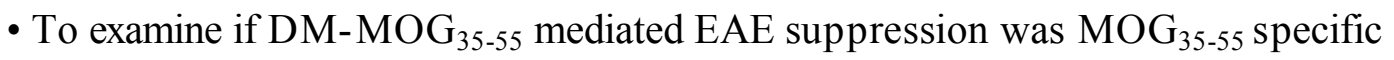

- To determine the role of bMDSCs in $\mathrm{DM}-\mathrm{MOG}_{35-55}$ mediated EAE suppression

- To optimise $\mathrm{DM}-\mathrm{MOG}_{35-55}$ treatment delivery 


\subsection{Results}

\subsubsection{DM-MOG $35-55$ suppressed MOG $_{35-55}$ induced EAE in C57BL/6J mice}

In order to examine whether the administration of $\mathrm{DM}-\mathrm{MOG}_{35-55}$ could suppress EAE, $\mathrm{DM}_{-} \mathrm{MOG}_{35-55}$ was added to the EAE inducing emulsion and injected into mice s.c. On day one, the recipient mice were injected with PTxn at a dose of 250 ng per mouse i.p. (this is subject to a similar administration regime as $\mathrm{SM}-\mathrm{MOG}_{35-55}$ used in Figure 3.8). As shown in Figure 5.1, DM-MOG $\mathrm{DO}_{35-55}$ treatment suppressed EAE in a dose-dependent manner. A low dose $(1 \mu \mathrm{g})$ of $\mathrm{DM}^{-M_{0}} \mathrm{G}_{35-55}$ only reduced the severity of EAE during the early stage. However, higher doses $(2 \mu \mathrm{g}$ and $5 \mu \mathrm{g})$ reduced both the disease severity and incidence.

From Figure 5.1, it can be seen that doses using 2 and $5 \mu \mathrm{g}$ of $\mathrm{DM}-\mathrm{MOG}_{35-55}$ resulted in similar levels of EAE suppression. Therefore, I used $2 \mu \mathrm{g}$ to treat mice in subsequent studies for the investigation of $\mathrm{DM}^{-} \mathrm{MOG}_{35-55}$ mediated EAE suppression mechanisms. Hereafter, the term "DM-MOG ${ }_{35-55}$ treatment" will refer to "2 $\mu \mathrm{g}$ of $\mathrm{DM}-\mathrm{MOG}_{35-55}$ added to the EAE inducing emulsion and delivered s.c. into mice on day zero".

As shown in Table 5.1, the DM-MOG $35-55$ treatment greatly reduced the incidence of EAE in the recipient mice. Mice treated with $\mathrm{DM}_{-} \mathrm{MOG}_{35-55}$ had a $20-50 \%$ EAE incidence in comparison with $100 \%$ disease incidence in the control mice $(50-80 \%$ protection). Although the $\mathrm{DM}-\mathrm{MOG}_{35-55}$ treatment did not protect all the recipient mice from EAE, as shown in Table 5.2, the mortality was markedly reduced in the treated mice that developed EAE. The DM-MOG $35-55$ treatment may have also slightly delayed the onset and reduced the maximum severity of EAE in the DM-MOG $35-55$ treated sick mice (although not statistically significant). 

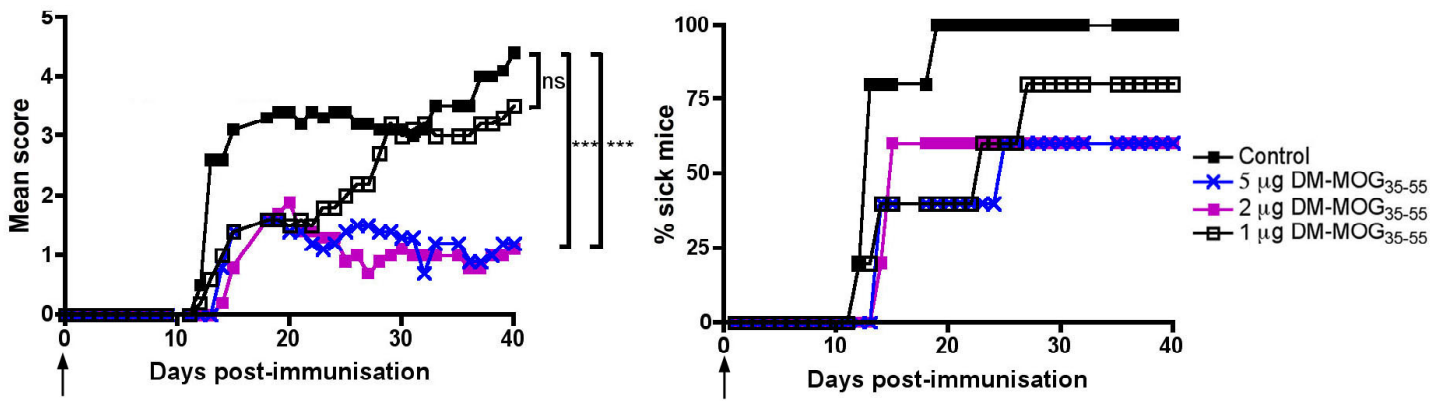

Figure 5.1 DM-MOG D5-55 suppressed EAE in a dose-dependent manner

Left graph, mean EAE scores of the C57BL/6J mice immunised with $\mathrm{MOG}_{35-55}$ emulsified in CFA plus 1,2 or $5 \mu \mathrm{g}$ of DM-MOG ${ }_{35-55}$. Mice immunised without the addition of DM-MOG ${ }_{35-55}$ were employed as the experimental controls. Right graph, \%sick mice. Significant differences are indicated, ${ }^{* * *} \mathrm{p}<0.0001$. ns, not significant. Analysis of statistical significance was performed using a one tailed Mann Whitney U test. Each group contained five mice. Arrows indicate the days of DM-MOG $\mathrm{MS}_{35}$ administration.

Table 5.1 Reproducibility of the treatment using DM-MOG $\mathbf{D S}_{35-55}$

$\mathrm{C} 57 \mathrm{BL} / 6 \mathrm{~J}$ mice were treated with EAE inducing emulsion plus $2 \mu \mathrm{g}$ of $\mathrm{DM}-\mathrm{MOG}_{35-55}$. Age- and sex-matched mice left untreated were employed as the experimental controls.

\begin{tabular}{|c|c|c|c|c|}
\hline $\begin{array}{c}\text { Experimental } \\
\text { Number }\end{array}$ & $\begin{array}{c}\text { Treated mice } \\
\text { (Sick/total) }\end{array}$ & $\begin{array}{c}\text { \% Sick of } \\
\text { treated mice }\end{array}$ & $\begin{array}{c}\text { Control mice } \\
\text { (Sick/total) }\end{array}$ & $\begin{array}{c}\text { \% Sick of } \\
\text { control mice }\end{array}$ \\
\hline $\mathbf{1}$ & $2 / 4$ & $50 \%$ & $5 / 5$ & $100 \%$ \\
\hline $\mathbf{2}$ & $2 / 5$ & $40 \%$ & $5 / 5$ & $100 \%$ \\
\hline $\mathbf{3}$ & $1 / 5$ & $20 \%$ & $5 / 5$ & $100 \%$ \\
\hline Total & $5 / 14$ & $35.71 \%$ & $15 / 15$ & $100 \%$ \\
\hline
\end{tabular}

Table 5.2 DM-MOG D5-55 treatment suppressed EAE

C57BL/6J mice were treated with EAE inducing emulsion plus $2 \mu \mathrm{g}$ of DM-MOG $\mathrm{M}_{35-55}$. Age- and sex-matched mice left untreated were employed as the experimental controls. The incidence of EAE, mortality, mean maximum score of each group \pm SD and mean day of onset \pm SD are shown. Significant differences are indicated, ns, not significant $(\mathrm{p}>0.05)$. Analysis of statistical significance was performed using a student $t$ test.

\begin{tabular}{|c|c|c|c|c|}
\hline Treatment & $\begin{array}{c}\text { Incidence of } \\
\text { EAE (\%) }\end{array}$ & $\begin{array}{c}\text { Mortality } \\
\mathbf{( \% )}\end{array}$ & $\begin{array}{c}\text { Mean maximum } \\
\text { severity of sick } \\
\text { mice }\end{array}$ & $\begin{array}{c}\text { Mean day of } \\
\text { onset of sick } \\
\text { mice }\end{array}$ \\
\hline Control & $15 / 15(100 \%)$ & $5 / 15(33.33 \%)$ & $4.04 \pm 0.74$ & $16.57 \pm 6.03$ \\
\hline DM-MOG $_{35-55}$ & $5 / 14(35.71 \%)$ & $0 / 14(0 \%)$ & $3.25 \pm 0.61^{\mathrm{ns}}$ & $18.33 \pm 6.56^{\mathrm{ns}}$ \\
\hline
\end{tabular}




\subsubsection{DM-MOG D5-55 treatment reduced cell infiltration into the CNS}

Next I investigated whether the $\mathrm{DM}-\mathrm{MOG}_{35-55}$ treatment prevented EAE by decreasing cellular infiltration into the CNS or by suppressing the disease after the cell entry into the CNS.

To assess CNS cellular infiltration, 40 days after EAE induction, the spinal cords of mice were sectioned and H\&E stained. As shown in Figure 5.2, DM-MOG $35-55$ treatment greatly reduced cell infiltration into the CNS. The spinal cords isolated from control mice showed massive cell infiltration in foci around the edges of the spinal cords (Figure 5.2B). In comparison, there was no obvious cellular infiltration into the spinal cords from $\mathrm{DM}-\mathrm{MOG}_{35-55}$ treated mice (Figure 5.2D). Thus, DM-MOG $35-55$ treatment prevented cell infiltration and/or accumulation into the CNS.
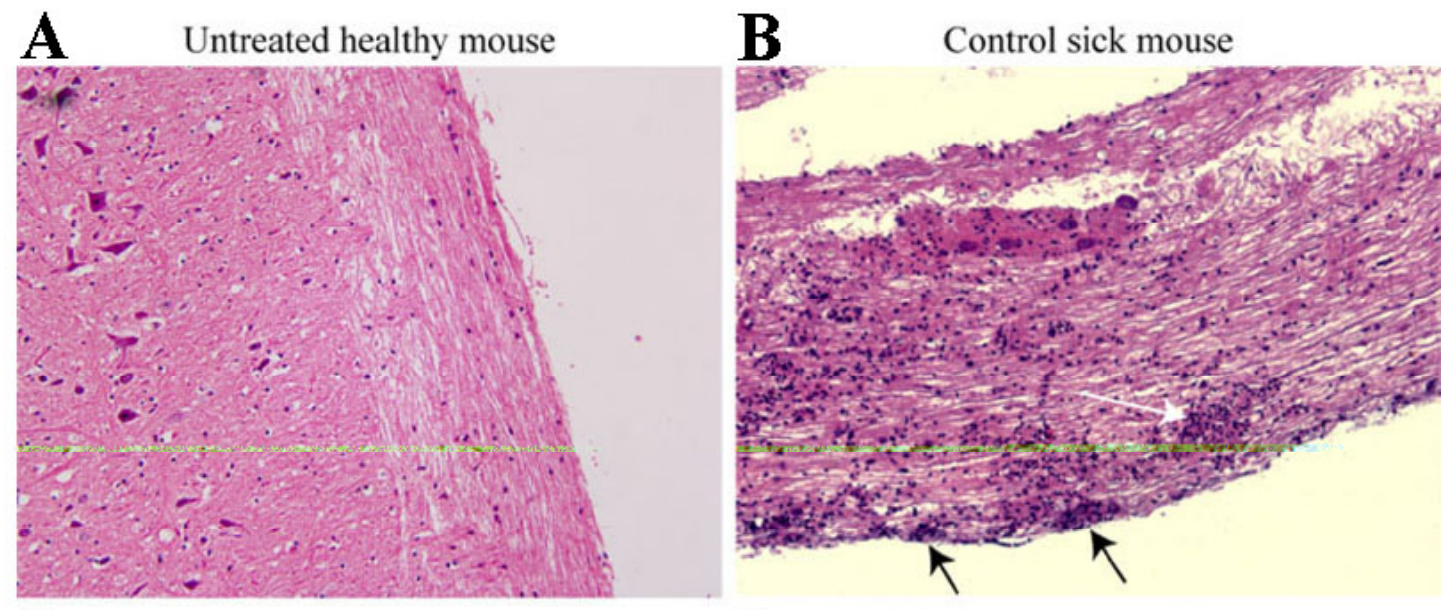

C DM-MOG $_{35.55}$ treated non-sick mouse
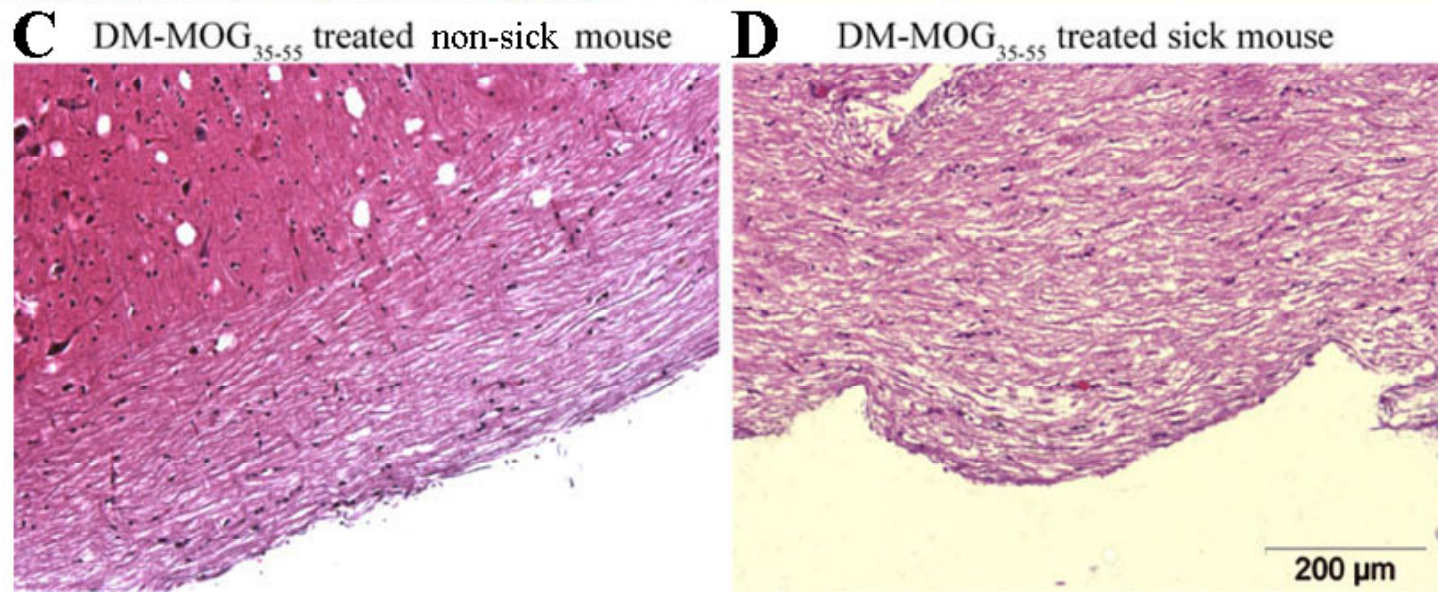

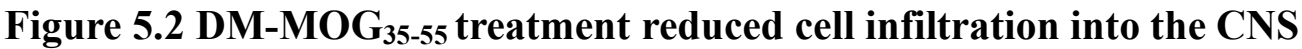

H\&E stained spinal cord sections from untreated healthy mouse (naïve mouse), control sick mouse, $\mathrm{DM}-\mathrm{MOG}_{35-55}$ treated (non-sick and sick) on day 40. One representative slide from each group is shown $(A=5, B=5, C=9, D=5)$. Arrows indicate the infiltrating lymphocytes. On the day of spinal cord removal, EAE scores of all the mice in the control group's were four, and that of $\mathrm{DM}-\mathrm{MOG}_{35-55}$ treated sick mice were between 1-2. 


\subsubsection{The $M O_{35-55}$ peptide conjugated to DM was necessary for EAE suppression}

Although DM-MOG $35-55$ exhibited a suppressive effect on EAE, it was not clear whether the $\mathrm{MOG}_{35-55}$ peptide conjugated to DM was necessary for this $\mathrm{EAE}$ suppression. To address this question, unconjugated DM was added to the EAE inducing emulsion and injected into mice s.c. on day zero. As shown in Figure 5.3, the administration of unconjugated DM did not suppress EAE, indicating the $\mathrm{MOG}_{35-55}$ peptide conjugated to DM was required for EAE suppression.

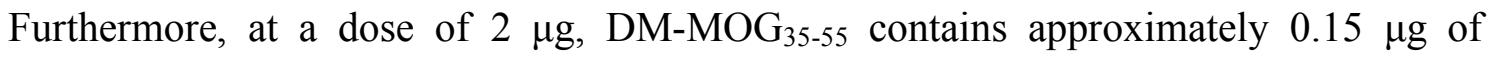
$\mathrm{MOG}_{35-55}$ peptide conjugated to DM. Mixing this dose of the $\mathrm{MOG}_{35-55}$ peptide to the EAE inducing emulsion that contained $50 \mu \mathrm{g}$ of $\mathrm{MOG}_{35-55}$ peptide did not alter either EAE scores or incidence in the recipient mice (data not shown) indicating that it was not the additional $\mathrm{MOG}_{35-55}$ peptide that induced disease suppression. Therefore, the conjugation of $\mathrm{DM}$ to the $\mathrm{MOG}_{35-55}$ peptide was required for the suppression of EAE.
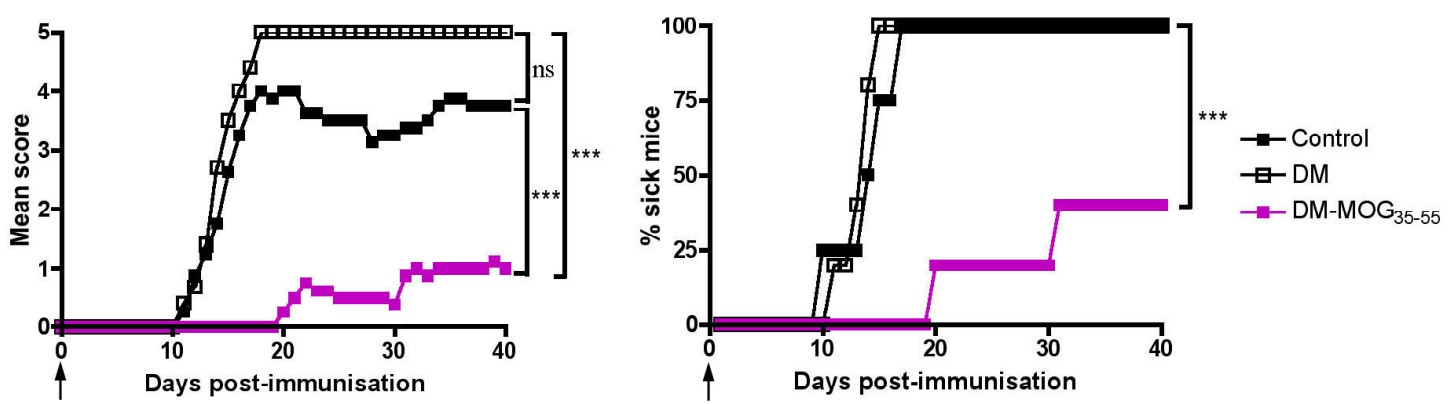

Figure 5.3 MOG $_{35-55}$ peptide conjugated to DM was necessary for EAE suppression Left graph, mean clinical scores of C57BL/6J mice immunised with $\mathrm{MOG}_{35-55}$ emulsified in CFA plus $2 \mu \mathrm{g}$ of DM or DM-MOG $35-55$. Right graph, \%sick mice. The development of clinical signs of EAE was monitored daily. Significant differences are indicated, $* * * p<0.0001$; ns, not significant. Analysis of statistical significance was performed using a one tailed Mann Whitney $U$ test. The DM and DM-MOG ${ }_{35-55}$ groups contained five mice and the control group contained four mice. SM-MOG $35-55$ treatment as shown in Figure 5.10 was also included in this experiment. However, results are not indicated here for clarity. Arrows indicate the days of $\mathrm{DM}-\mathrm{MOG}_{35-55}$ or DM administration.

\subsubsection{DM-MOG D5-55 $_{3}$ mediated suppression of EAE was MOG $_{35-55}$ specific}

To determine whether DM-MOG $35-55$ mediated suppression of EAE was $\mathrm{MOG}_{35-55}$ specific, 40 days after DM-MOG $35-55$ treatment, draining lymph node (DLN) cells of the treated mice were pooled and restimulated in vitro with different stimuli including $\mathrm{MOG}_{35-55}$. The cells' proliferative responses (Section 5.3.4.1) and cytokine production (Section 5.3.4.2) were measured. In order to investigate why DM-MOG $35-55$ treatment protected some mice, but not others, the DM-MOG $35-55$ treated mice were further 
divided into sick and non-sick groups according to their disease status. DLN cells from the untreated immunised mice, all of which demonstrated EAE symptom, were used as the experimental controls.

\subsubsection{DM-MOG D5-55 treatment specifically reduced DLN cells' proliferation in response to $\mathrm{MOG}_{35-55}$ restimulation in vitro}

As shown in Figure 5.4, DLN cells from the DM-MOG $35-55$ treated mice exhibited reduced proliferation in response to $\mathrm{MOG}_{35-55}$ restimulation in vitro compared to that from the control mice. In particular, DLN cells from the DM-MOG $\mathrm{DOG}_{35-55}$ treated non-sick mice showed a marked reduction in cell proliferation in response to $\mathrm{MOG}_{35-55}$. In contrast, the proliferative responses to anti-CD3 antibody (Clone 145-2c11) and mycobacterium-derived purified protein derivative (PPD), which was contained in CFA, were similar for all the mice. None of the DLN cells responded to the irrelevant control peptide $\mathrm{OVA}_{323-339}$.
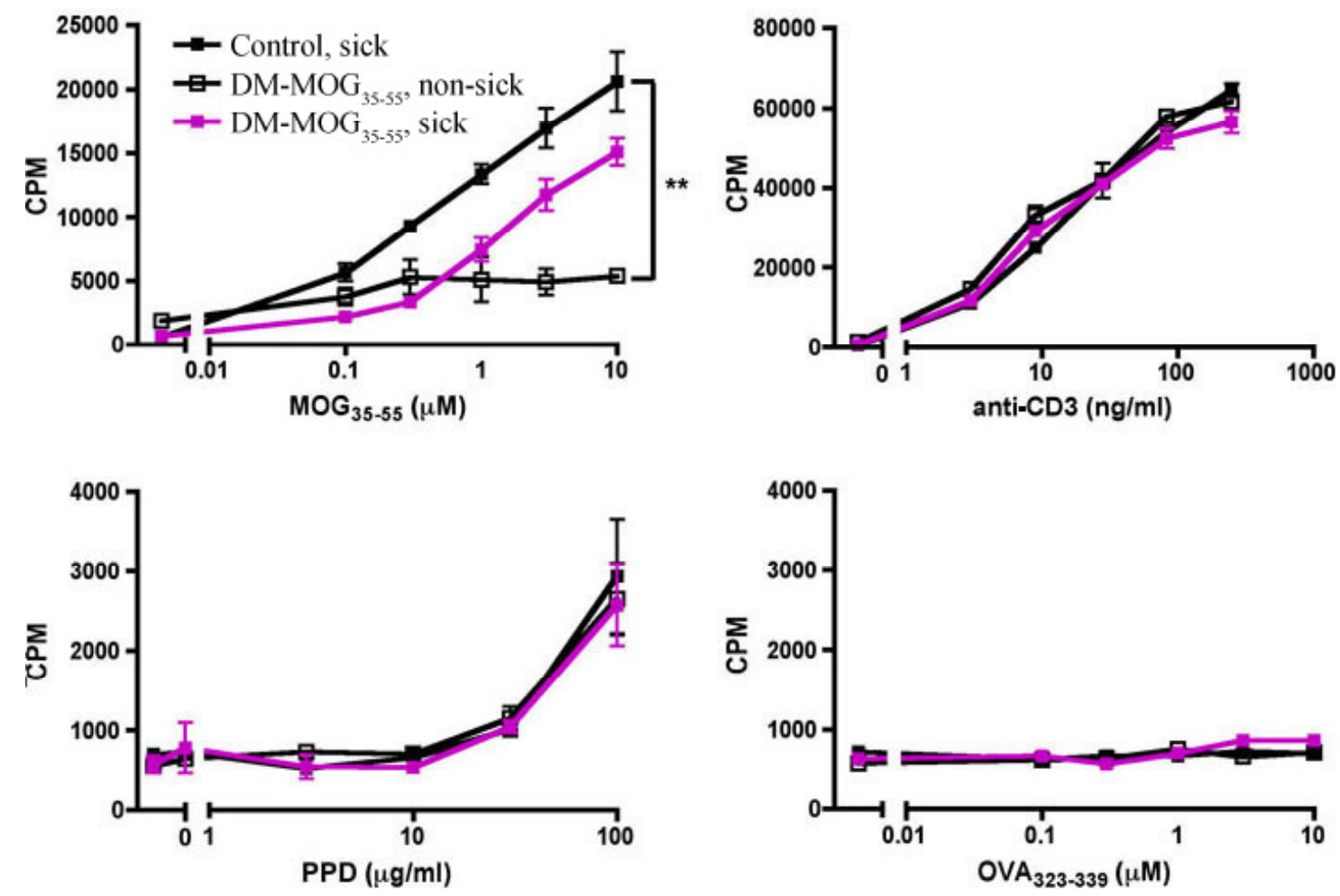

Figure 5.4 DM-MOG D5-55 treatment specifically reduced DLN cells' proliferation in response to $\mathrm{MOG}_{35-55}$ restimulation in vitro

DLNs (inguinal lymph nodes) were collected 40 days after EAE induction. The DM-MOG $35-55$ treated mice were divided into sick and non-sick groups. Untreated immunised mice were employed as the experimental controls. Cells were pooled and restimulated with either $\mathrm{MOG}_{35-55}, 2 \mathrm{c} 11$, PPD or OVA $323-339$ for 72 hours. $\left[{ }^{3} \mathrm{H}\right]$-thymidine was added to the culture and incubated for a further 16 hours. Significant differences are indicated, $* * \mathrm{p}<0.01$. Analysis of statistical significance was performed using a one tailed Mann Whitney $U$ test. Results are representative of two independent experiments. 


\subsubsection{DM-MOG D5-55 treatment reduced DLN cells' production of IL-17 and IFN- $\gamma$} in response to $\mathrm{MOG}_{35-55}$ restimulation in vitro

Supernatant from the cell cultures described in Figure 5.4 were collected and the cytokine levels were assessed. As shown in Figure 5.5, DLN cells from the DM-MOG ${ }_{35-55}$ treated mice showed reduced production of the cytokines IFN- $\gamma$ and IL-17 in response to $\mathrm{MOG}_{35-55}$ restimulation in vitro, compared to that from the control mice. In particular, DLN cells from the DM-MOG $35-55$ treated non-sick mice showed a marked reduction in the production of IFN- $\gamma$ and IL-17 in response to $\mathrm{MOG}_{35-55}$. The production of these two cytokines in response to PPD and anti-CD3 antibody was similar in all the mice. The levels of TNF- $\alpha$, IL-4 and IL-10 were low in all the samples and there was no difference observed between groups (Figure 5.6). The specific impairment of DLN cells' effector function in response to $\mathrm{MOG}_{35-55}$ but not to anti-CD3 or PPD indicated that the $\mathrm{DM}_{-} \mathrm{MOG}_{35-55}$ treatment induced an in vivo $\mathrm{MOG}_{35-55}$-specific suppression of $\mathrm{T}$ cell response and this antigen-specific suppression did not compromise the immune system to respond to other stimuli. 


\section{IL-17}
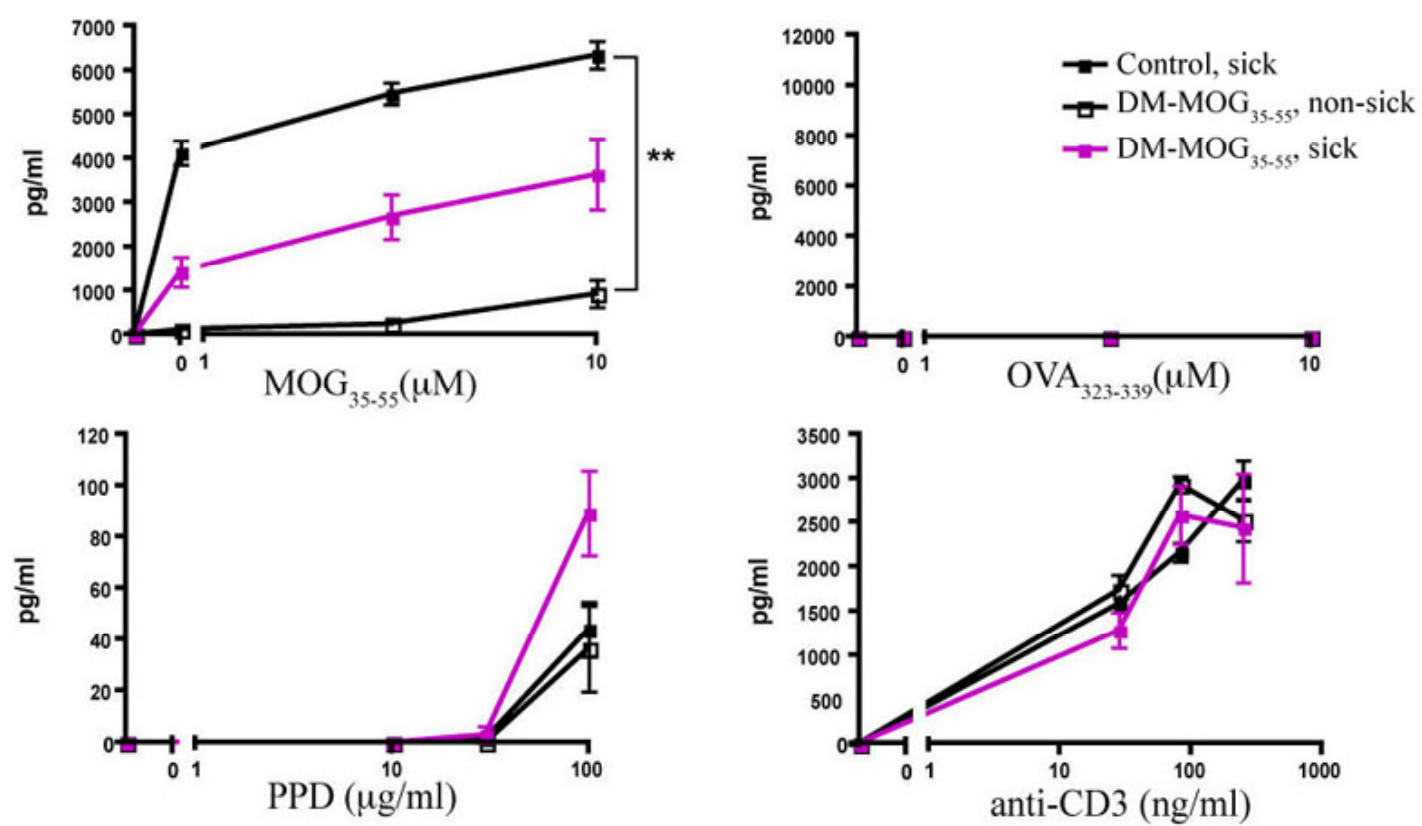

\section{IFN- $\gamma$}
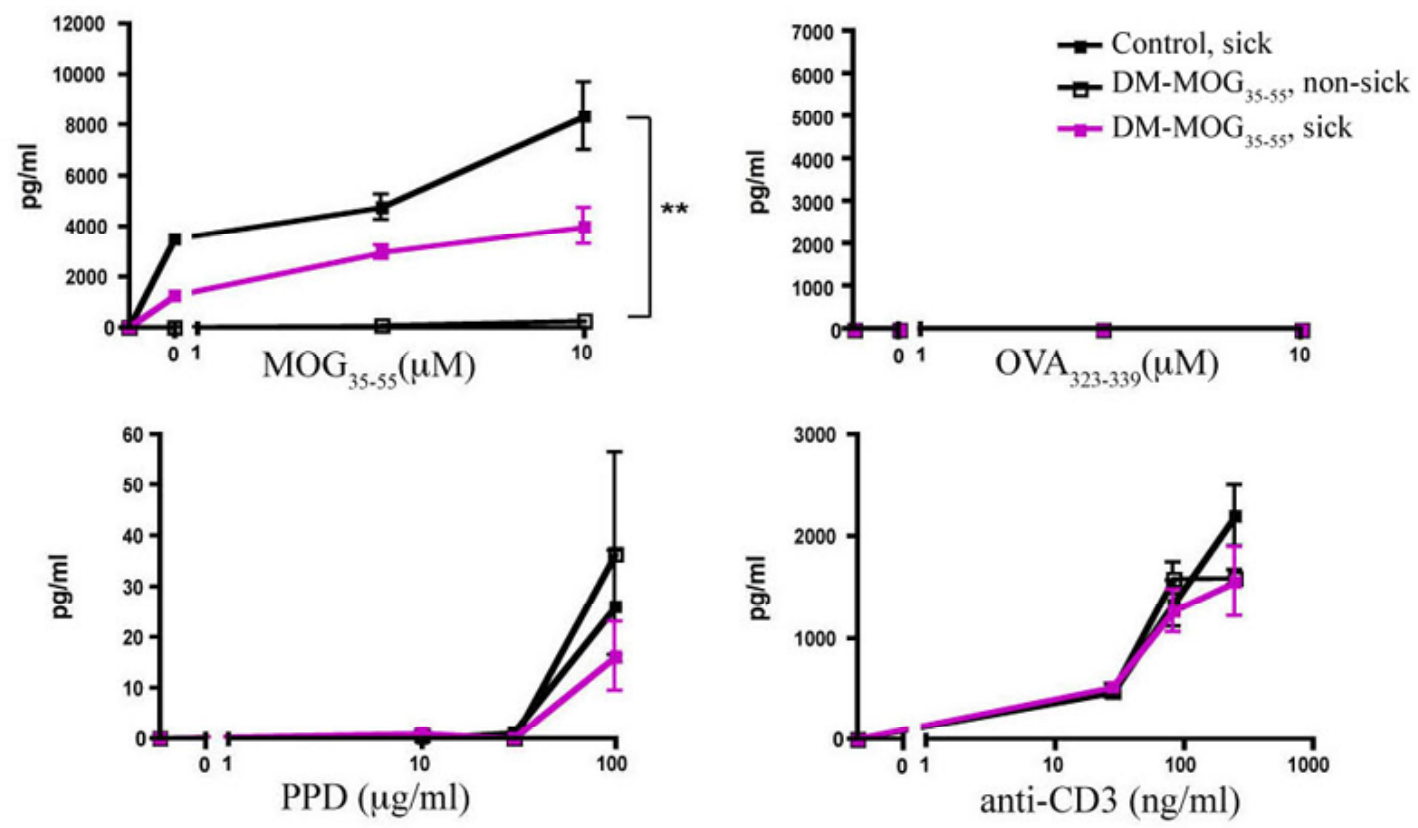

Figure 5.5 DM-MOG D5-55 treatment reduced the DLN cells' production of IL-17 and IFN- $\gamma$ in response to $M O_{35-55}$ restimulation in vitro

From the cell cultures as shown in Figure 5.4, supernatant was collected at 72 hours, and the levels of IL-17 and IFN- $\gamma$ were measured. Results are shown as the mean of triplicate wells \pm SEM. Significant differences are indicated, ${ }^{* *} \mathrm{p}<0.01$. Analysis of statistical significance was performed using a one tailed Mann Whitney U test. Results are representative of two independent experiments. 

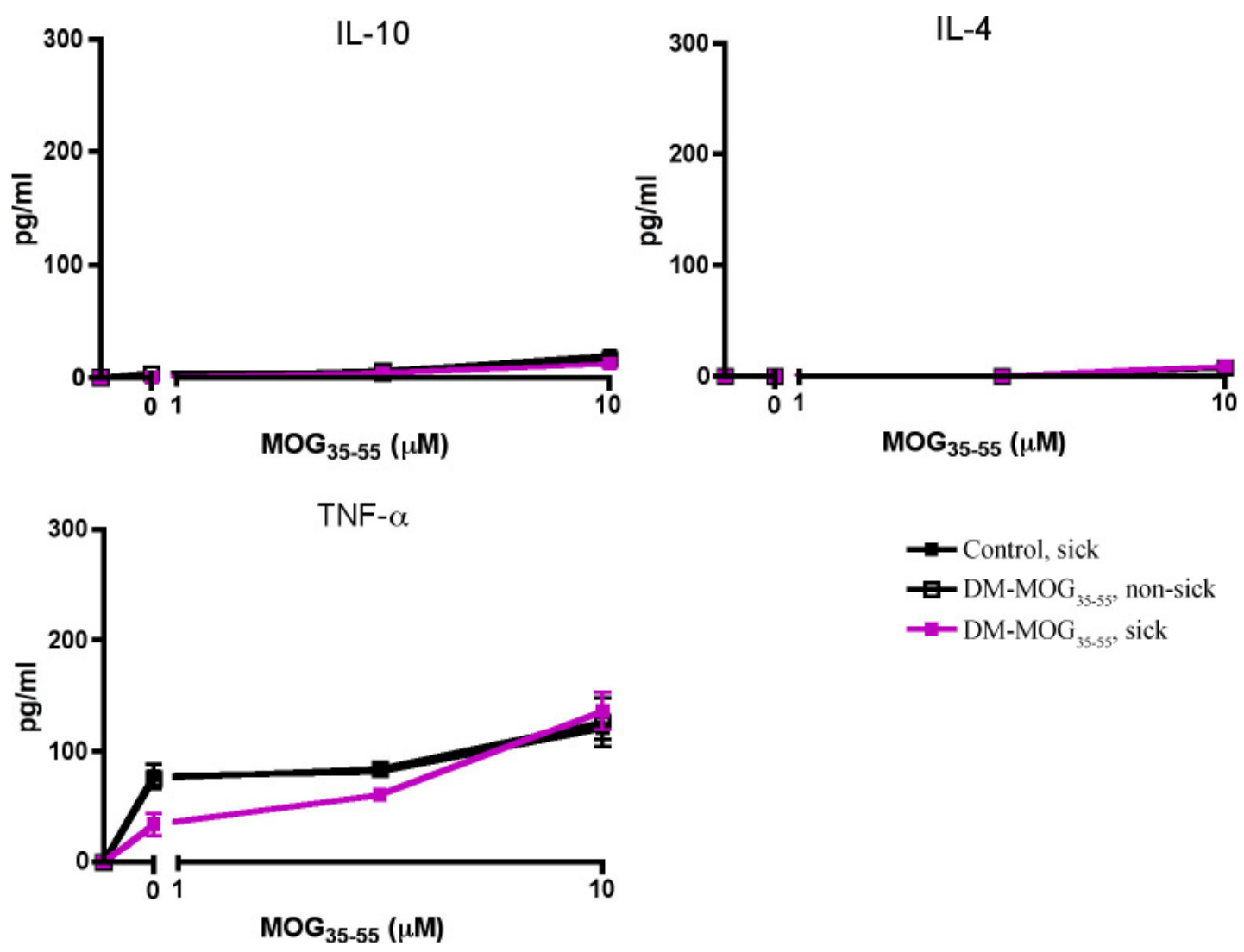

Figure 5.6 DM-MOG DS-55 $_{3}$ treatment did not alter IL-10, IL-4 or TNF- $\alpha$ production by DLN cells in response to the $\mathrm{MOG}_{35-55}$ restimulation in vitro

From the cell culture as shown in Figure 5.4, supernatant was collected at 72 hours, and the levels of IL-10, IL-4 and TNF- $\alpha$ were measured. Results are shown as the mean of triplicate wells \pm SEM. Results are representative of two independent experiments.

\subsection{5 bMDSCs played a key role in $\mathrm{DM}^{-M_{0 G}} \mathrm{G}_{35-55}$-mediated suppression of EAE}

Although DM-MOG $35-55$ treatment endowed a $\mathrm{MOG}_{35-55}$ specific suppression of $\mathrm{T}$ cell

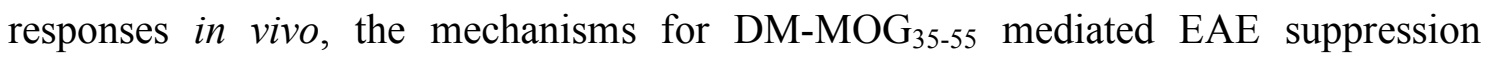
remained to be determined. Because DM bound to the blood suppressor cells bMDSCs in vivo, I hypothesised that bMDSCs play an important role in $\mathrm{DM}-\mathrm{MOG}_{35-55}$ mediated EAE suppression.

\subsubsection{Adoptive transfer of the bMDSCs from DM-MOG $_{35-55}$ treated mice suppressed EAE}

A direct way to verify whether bMDSCs were important for DM-MOG $35-55$ mediated EAE suppression was to examine whether adoptive transfer of the bMDSCs from DM-MOG ${ }_{35-55}$ treated mice could suppress the disease in the recipient mice. As shown in Figure 5.7, adoptive transfer of bMDSCs from $\mathrm{DM}-\mathrm{MOG}_{35-55}$ treated mice conferred protection to the recipients. In contrast, the bMDSCs from naïve mice or the mice 
treated with DM did not transfer EAE protection. Together, these results confirmed that

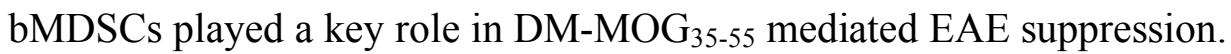
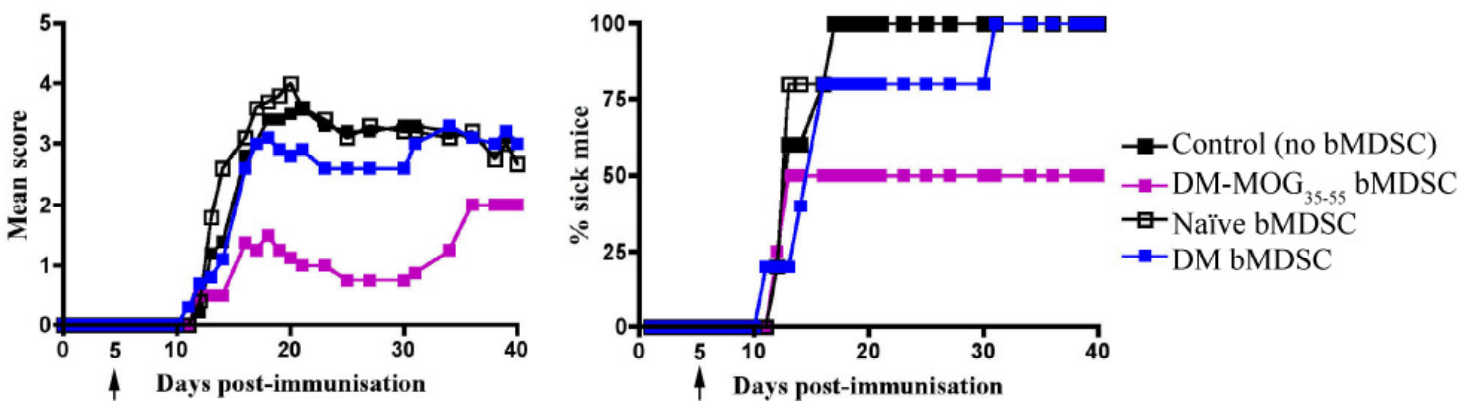

Figure 5.7 Adoptive transfer of the bMDSCs from DM-MOG 35-55 treated mice suppressed EAE

Mice were either treated with $2 \mu \mathrm{g}$ of DM- $\mathrm{MOG}_{35-55}$ or $2 \mu \mathrm{g}$ of DM mixed in EAE inducing emulsion, or left untreated. On day five, bMDSCs were purified from these treated mice (DM-MOG ${ }_{35-55}$ MDSC or DM MDSC) or naïve mice (Naïve MDSC), and adoptively transferred into the mice induced with EAE five days earlier ( $2 \times 10^{5}$ bMDSCs per mouse). As the experimental control, mice were injected with PBS i.v. on day five after EAE induction (No bMDSC). Left graph, clinical scores. Right graph, \%sick mice. The adoptive transfer of the bMDSCs from DM- $\mathrm{MOG}_{35-55}$ treated or naïve mice for EAE suppression have been done twice and that from DM has been done once. Arrows indicate the days of bMDSC adoptive transfer.

\subsubsection{2 bMDSCs depletion methods}

The traditional way to examine whether a treatment is dependent on a population of cells is to deplete the cells prior to the treatment. I considered three methods for bMDSC depletion: the administration of cytochrome c, neutralising antibodies (including CD11b, Gr-1 and Ly6C) or liposome-encapsulated dichloromethylene disphosphonate (clodronate liposome).

Cytochrome $\mathrm{c}$ is a soluble mitochondrial protein and is capable of recycling through the cytosol and killing cells by apoptotic protease-activating factor 1 (Apaf-1)-dependent apoptosis $^{218}$. Once released into the cytosol, cytochrome $\mathrm{c}$ binds to its partner protein Apaf-1 to form an apoptosome and causes a series of downstream effector caspases, eventually resulting in cell death ${ }^{218}$. Injecting mice with $1.67,5$ or $15 \mathrm{mg}$ horse cytochrome c i.v. (Sigma-Aldrich, USA) resulted in a decrease in $\mathrm{CD} 8^{+} \mathrm{DCs}$ and inhibition of cross-presentation as observed previously ${ }^{218}$. However, bMDSCs were not depleted (unpublished data, Dickgreber N.), indicating that the bMDSC population is different from $\mathrm{CD} 8^{+} \mathrm{DCs}$ for antigen cytosolic transfer. 
I also considered depleting bMDSCs using antibodies. However, as bMDSCs and neutrophils have many common surface markers, using neutralising antibodies such as anti-CD11b, anti-Gr-1 or anti-Ly6C as the bMDSC depleting agent may be confounded by the unintended depletion of neutrophils. As described in Chapter 4, neutrophils express high levels of all the above three antigens. Using any of these antibodies would lead to a depletion of neutrophils, which are critical for EAE development ${ }^{219-222}$. Although anti-Gr-1 antibody has been used to deplete splenic MDSCs in a tumour model $^{72}$, it is inappropriate to apply this method to the current study, as bMDSCs express much lower levels of Gr-1 than the MDSCs described in tumour models $^{71,72,223,224}$. Therefore, depleting bMDSCs by using antibodies was not pursued.

Intravenous injection of clodronate, an intracellular toxin, using liposome as vehicles has been widely used to deplete phagocytotic cells such as macrophages in vivo ${ }^{225-229}$. Once taken up by phagocytes, the liposomal membranes are digested by phospholipases in the lysosomes and clodronate is released triggering cell death ${ }^{225}$. As shown in Figure 5.8, 18 hours after i.v. administration of clodronate liposomes, the majority of the bMDSCs (defined as $\mathrm{SSC}^{\text {low }} \mathrm{CD} 11 \mathrm{~b}^{+} \mathrm{F} 4 / 80^{+}$) were depleted from the blood whereas the neutrophil numbers (defined as $\mathrm{SSC}^{\mathrm{hi}} \mathrm{CD} 11 \mathrm{~b}^{+}$) were unchanged. 
A

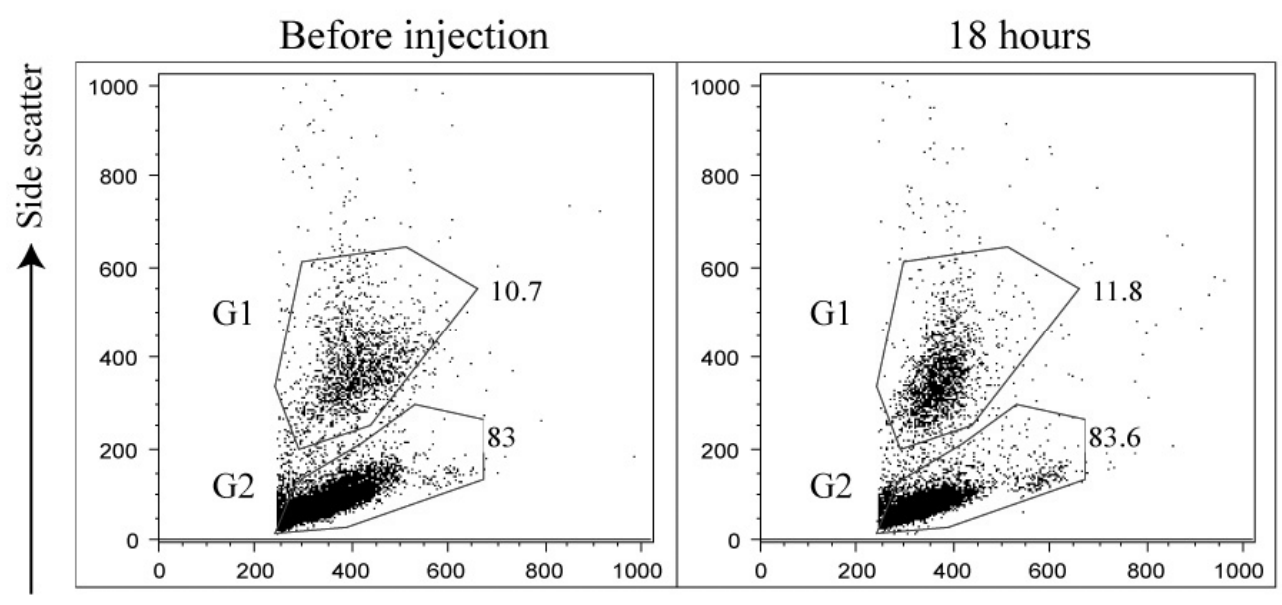

Forward scatter

B

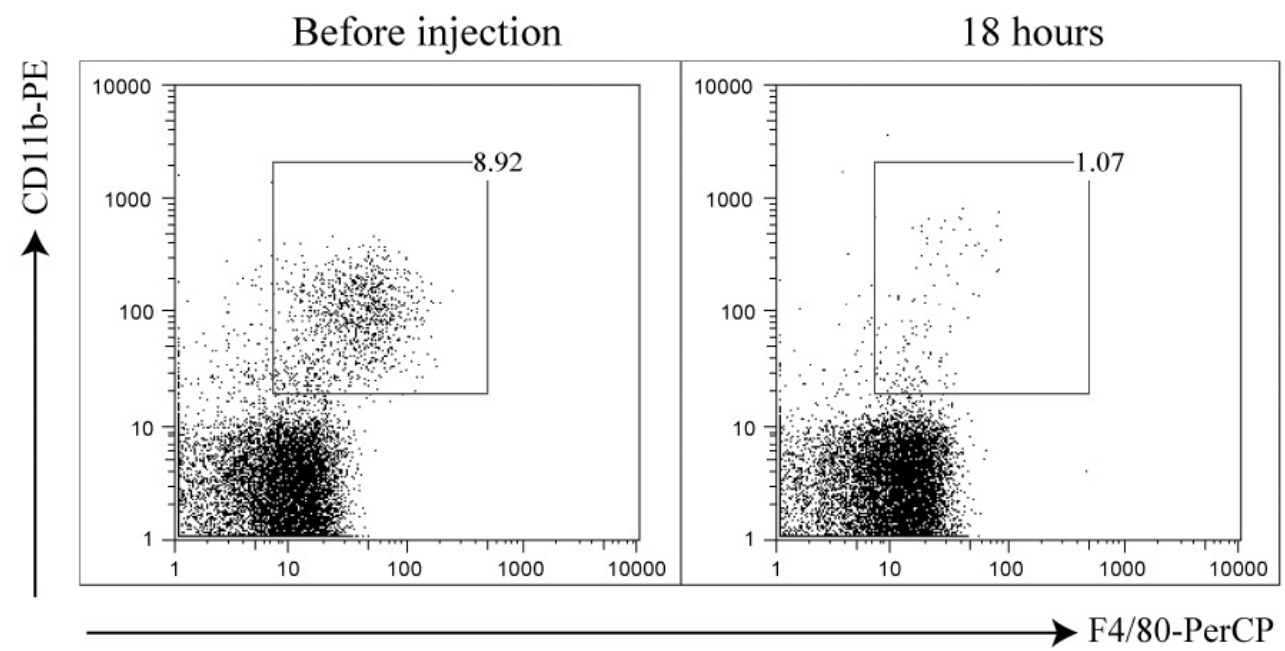

Figure 5.8 Depletion of the bMDSCs by i.v. injection of clodronate liposomes

Clodronate liposomes were i.v. injected into C57BL/6J mice. Before and 18 hours after the clodronate liposomes injection, tail blood cells from the mice were analysed by flow cytometry. A, blood cell profiles of forward and side scatter. Neutrophils were located in G1. bMDSCs and other leukocytes such as $\mathrm{T}$ cells are located in G2. B, CD11b and F4/80 profiles of the cells gated in G2. The bMDSCs were shown as the $\mathrm{CD} 11 \mathrm{~b}^{+} \mathrm{F} 4 / 80^{+}$cells indicated in the gates. The numbers indicate the percentages of gated cells.

After confirming depletion of bMDSCs by clodronate liposomes, I investigated whether the depletion of bMDSCs enhanced the $\mathrm{MOG}_{35-55}$ specific $\mathrm{CD}^{+} \mathrm{T}$ cell response in vivo. CFSE labelled 2D2 transgenic lymph node cells were adoptively transferred into CD45.1 congenic recipients with or without clodronate liposome treatment. One day after the lymph node cell transfer, the recipient mice were injected with the $\mathrm{MOG}_{35-55}$ peptide. Proliferation of the transferred $2 \mathrm{D} 2 \mathrm{CD}^{+} \mathrm{T}$ cells was assessed by flow cytometry on day five. As shown in Figure 5.9, unexpectedly, in mice treated with clodronate liposomes, $\mathrm{CD} 4^{+} \mathrm{T}$ cells proliferation was markedly reduced. This indicates that the injection of clodronate liposomes may also deplete APCs as observed 
previously $^{225,226}$ or have $\mathrm{T}$ cell toxicity, which downregulated $\mathrm{CD}^{+}$lymphocyte responses in vivo.

In conclusion, although the administration of clodronate liposomes depleted bMDSCs, this administration also markedly reduced $\mathrm{CD}^{+} \mathrm{T}$ cell responses in vivo, which is key for EAE development ${ }^{112}$. Therefore, using clodronate liposomes to investigate the role of bMDSCs in DM-MOG $35-55$ mediated EAE suppression was abandoned.

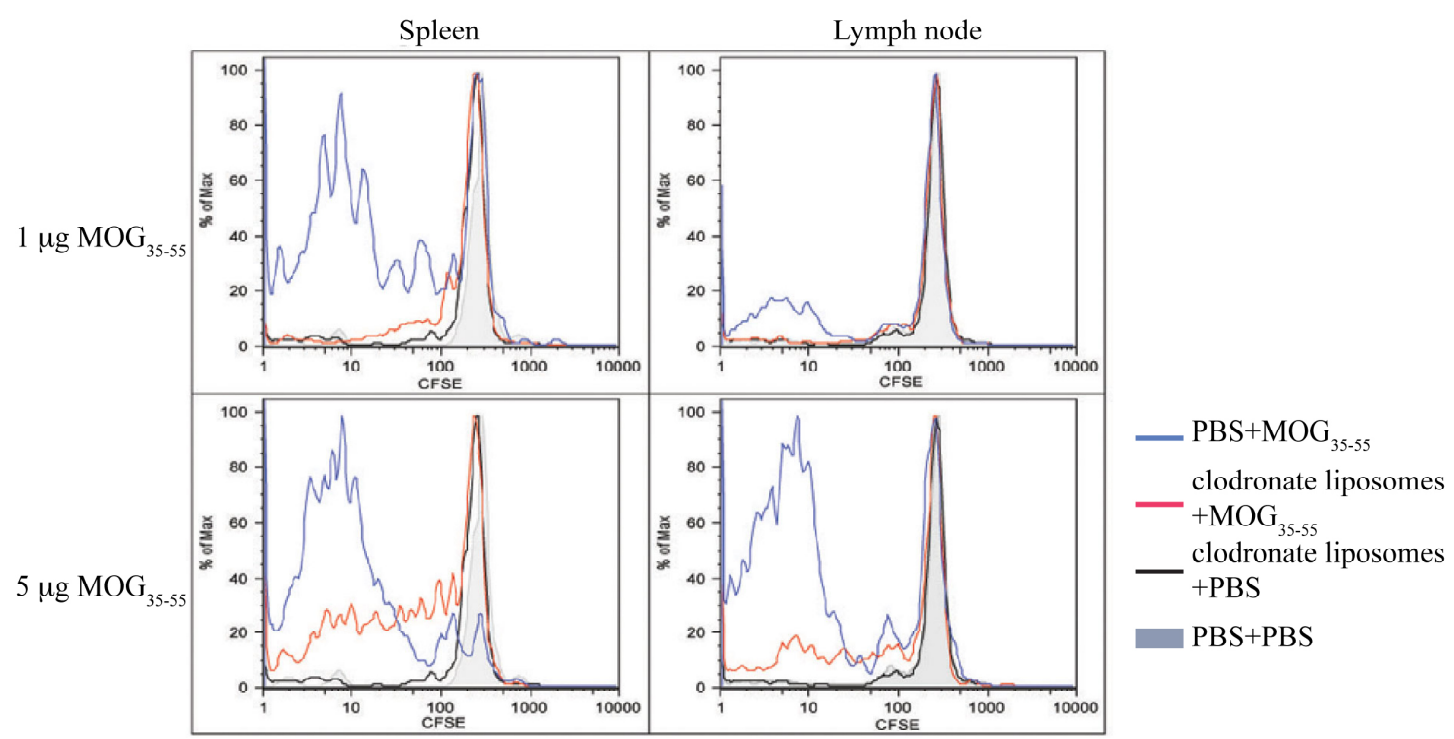

Figure 5.9 The administration of clodronate liposomes diminished $2 \mathrm{D} 2 \mathrm{CD4}^{+}$cell proliferation to $\mathrm{MOG}_{35-55}$ in vivo

CFSE labelled 2D2 transgenic lymph node cells were adoptively transferred into congenic CD45.1 recipients $\left(5 \times 10^{6}\right.$ cells per mouse). Clodronate liposomes or PBS were i.v. injected into the other side of the tail vein of the recipient mice. Eighteen hours after cell transfer, the recipient mice were injected with 1 or $5 \mu \mathrm{g}$ of $\mathrm{MOG}_{35-55}$ as indicated. Proliferation of the CFSE labelled $\mathrm{CD}^{+} \mathrm{T}$ cells was assessed by flow cytometry five days after the initiation of the experiment. Cells were gated on $\mathrm{CD} 4{ }^{+} \mathrm{CD} 45.2^{+}$cells.

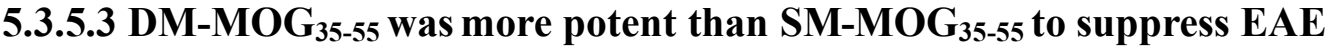

On the basis that DM demonstrated an enhanced binding capability to bMDSCs

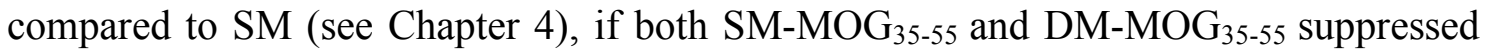
EAE via bMDSCs, DM-MOG $35-55$ would be expected to be more potent than

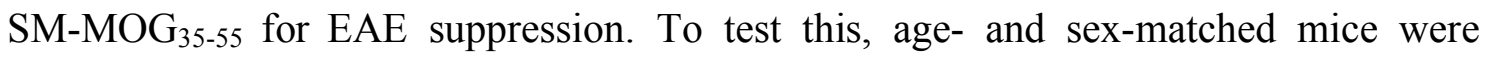
induced with EAE and administered with either $\mathrm{DM}-\mathrm{MOG}_{35-55}$ or $\mathrm{SM}-\mathrm{MOG}_{35-55}$ in parallel. As shown in Figure 5.10, DM-MOG ${ }_{35-55}$ exhibited enhanced EAE suppression compared to $\mathrm{SM}-\mathrm{MOG}_{35-55}$. This also provided evidence for the role of bMDSCs in mSAg-MOG $35-55$ mediated EAE suppression. 

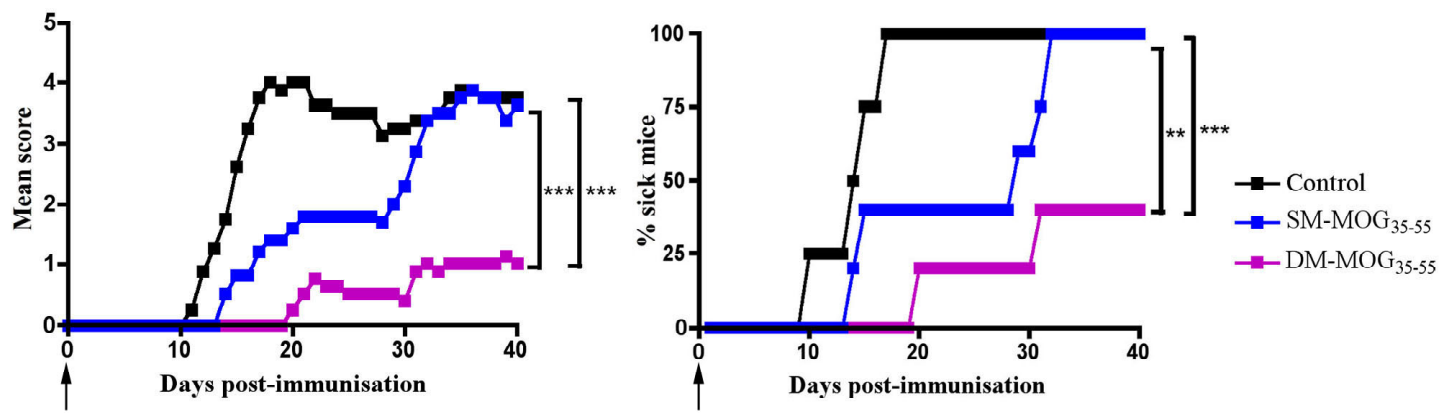

Figure 5.10 DM-MOG D5-55 was more potent than $\mathrm{SM}^{-M_{0}} \mathrm{G}_{35-55}$ to suppress $M_{35-55}$ induced EAE in C57BL/6J mice

C57BL/6J mice were immunised with $50 \mu \mathrm{g}$ of $\mathrm{MOG}_{35-55}$ emulsified in CFA plus $2 \mu \mathrm{g}$ of DM-MOG $\mathrm{MOG}_{35-55}$ or $2 \mu \mathrm{g}$ of SM-MOG ${ }_{35-55}$. Mice immunised with $50 \mu \mathrm{g}$ of $\mathrm{MOG}_{35-55}$ emulsified in CFA were employed as the experimental controls. On day one, all the mice were treated with $250 \mathrm{ng}$ PTxn i.p. Left graph, mean clinical scores. Right graph, \% sick mice. Significant differences are indicated, $* * * p<0.0001,{ }^{* *} p<0.01$. Analysis of statistical significance was performed using a one tailed Mann Whitney $U$ test. The SM-MOG $35-55$ and $\mathrm{DM}-\mathrm{MOG}_{35-55}$ groups contained five mice, and the control group contained four mice. One of the mice from the $\mathrm{SM}-\mathrm{MOG}_{35-55}$ group was culled on day 30 due to peritonitis. DM treatment as shown in Figure 5.3 was also included in this experiment; however, results are not indicated here for clarity. Arrows indicate the days for $\mathrm{DM}-\mathrm{MOG}_{35-55}$ or $\mathrm{SM}-\mathrm{MOG}_{35-55}$ administration.

\subsubsection{DM-MOG D5-55 treatment restored bMDSCs' in vitro suppressive capability}

To test whether $\mathrm{DM}^{-\mathrm{MOG}_{35-55}}$ treatment had a direct effect on bMDSCs' suppressive capability, I immunised mice with either the EAE inducing emulsion plus $2 \mu \mathrm{g}$ of

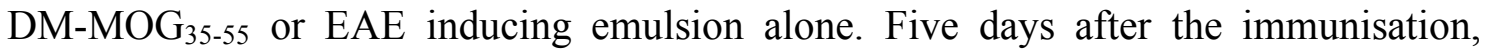
bMDSCs were purified and a $\mathrm{T}$ cell suppression assay was set up to compare the bMDSCs' suppressive capability. As shown in Figure 5.11, surprisingly, the bMDSCs from mice immunised with EAE inducing emulsion showed a reduced in vitro suppression of $\mathrm{T}$ cell proliferation compared to the bMDSCs derived from naïve mice. In contrast, the addition of $\mathrm{DM}-\mathrm{MOG}_{35-55}$ to the EAE inducing emulsion restored this suppression. This result confirmed that $\mathrm{DM}-\mathrm{MOG}_{35-55}$ treatment had a direct effect on bMDSCs' suppressive capability. 


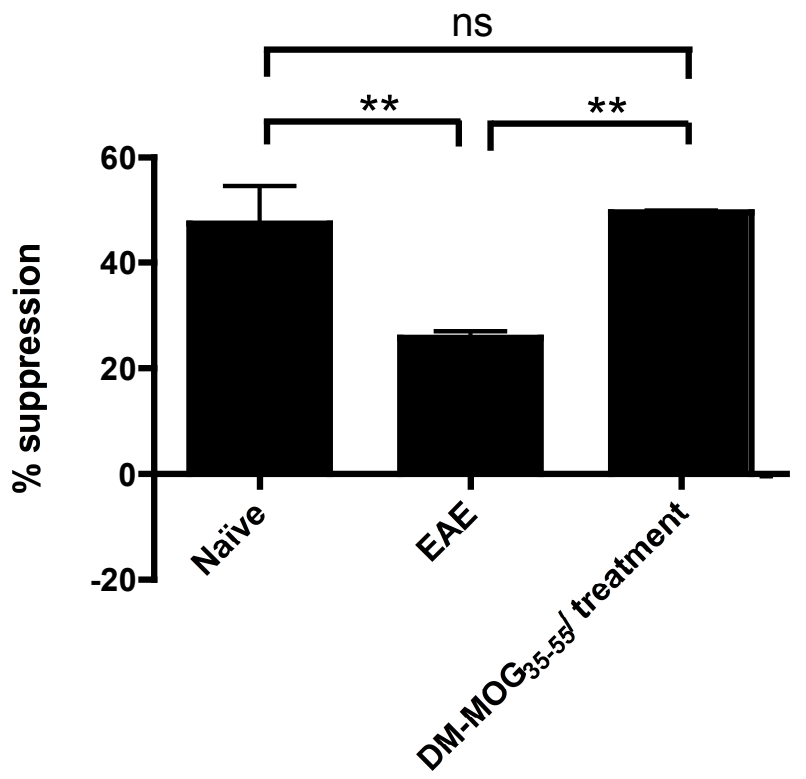

Figure 5.11 DM-MOG D5-55 treatment restored the bMDSCs' in vitro suppressive capability

Mice were treated with either EAE inducing emulsion only (EAE), emulsion plus $2 \mu \mathrm{g}$ of DM-MOG $35-55$ (DM-MOG $35-55 /$ treatment) or left untreated (Naïve). On day one, $250 \mathrm{ng}$ PTxn was injected into mice i.p. On day five, bMDSCs were purified to set up a T cell suppression assay. Fifty thousand lymph node cells from naïve mice were incubated with or without bMDSCs from the three groups (at a 1:1 ratio) in the presence of 25,000 expander beads in a 96-well round-bottom plate. Twenty-four hours later, $\left[{ }^{3} \mathrm{H}\right]$-thymidine was added to the cell culture and incubated for a further 16 hours. Results are shown as the average of triplicate wells \pm SEM. Significant differences are indicated, $* * p<0.01$. ns, not significant. Analysis of statistical significance was performed using a one tailed Mann Whitney U test.

\subsubsection{Optimisation of ${\mathrm{DM}-M O \mathrm{G}_{35-55}}_{\text {treatment delivery }}$}

In order to optimise therapeutic outcomes in EAE by the DM-MOG $\mathrm{B}_{35-55}$ treatment, different combinations of routes, doses and timing for the delivery of DM-MOG $35-55$ were examined.

\subsubsection{1 i.v. injection of DM-MOG ${ }_{35-55}$ did not suppress EAE}

Because i.v. administration of DM enhanced the in vitro suppressive performance of the bMDSCs (Figure 4.22), it brought the hope that $\mathrm{DM}^{-\mathrm{MOG}_{35-55}}$ i.v. treatment might suppress EAE. However, as shown in Figure 5.12, i.v. injection of $5 \mu \mathrm{g}$ of DM-MOG $35-55$ one day before EAE induction did not protect mice against the disease.

Next, I trialed different administration regimes (timing, dosage, administrative route and adjuvant) as listed in Appendix 2. From these trials, I determined that s.c. delivery of DM-MOG ${ }_{35-55}$ with EAE inducing emulsion was the most efficient administration route. 

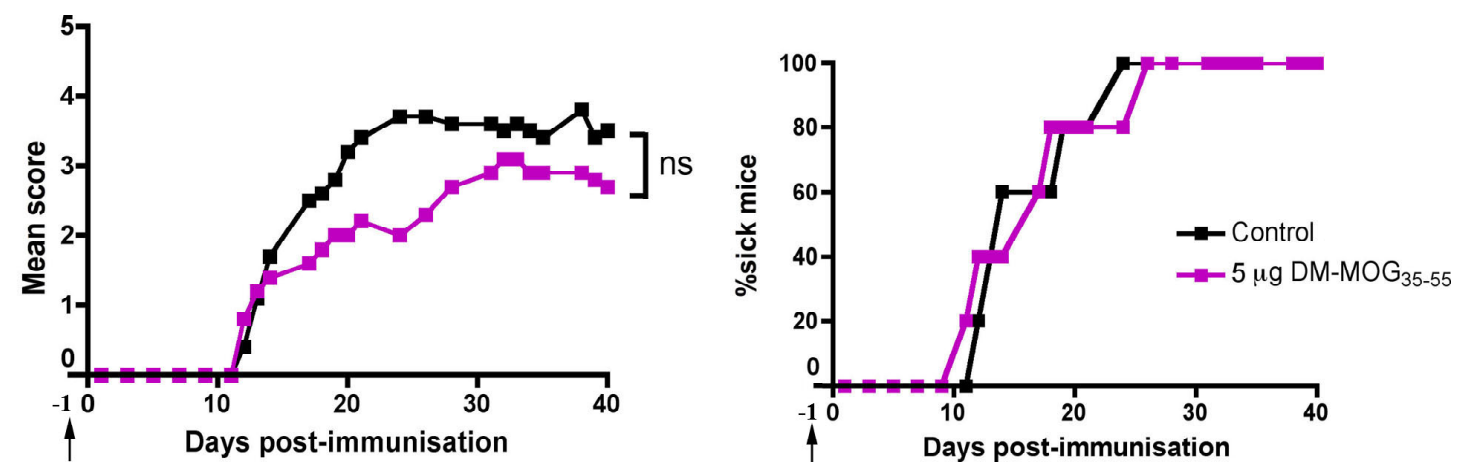

Figure 5.12 i.v. injection of DM-MOG ${ }_{35-55}$ did not suppress EAE

C57BL/6J mice were either i.v. injected with $5 \mu \mathrm{g}$ of DM-MOG $35-55$ or left untreated (control group) one day before EAE induction. Left graph, mean scores of EAE. Right graph, \%sick mice. Each group contained five mice. Significant difference is indicated, ns, not significant. Analysis of statistical significance was performed using a one tailed Mann Whitney U test.

\subsubsection{2 s.c. injection of DM-MOG ${ }_{35-55}$ in CFA or IFA on the neck protected mice against EAE}

As optimal treatment with $\mathrm{DM}-\mathrm{MOG}_{35-55}$ required s.c. administration at the site of initial immunisation, it was possible that the $\mathrm{DM}-\mathrm{MOG}_{35-55}$ treatment affected the priming of encephalitogenic $\mathrm{T}$ cells directly. In order to develop a clinically relevant therapy and investigate whether the $\mathrm{DM}-\mathrm{MOG}_{35-55}$ treatment affected the priming of encephalitogenic $\mathrm{T}$ cells, mice were treated with $2 \mu \mathrm{g}$ of $\mathrm{DM}-\mathrm{MOG}_{35-55}$ emulsified in CFA on the back of the neck. As shown in Figure 5.13, s.c. injection of $2 \mu \mathrm{g}$ of DM-MOG ${ }_{35-55}$ emulsified in CFA on the back of the neck suppressed EAE in the recipient mice, indicating that the suppression mediated by $\mathrm{DM}-\mathrm{MOG}_{35-55}$ was systemic. However, the suppression of EAE by this administration route was not as marked as the coimmunisation delivery system, suggesting the involvement of localised effects.
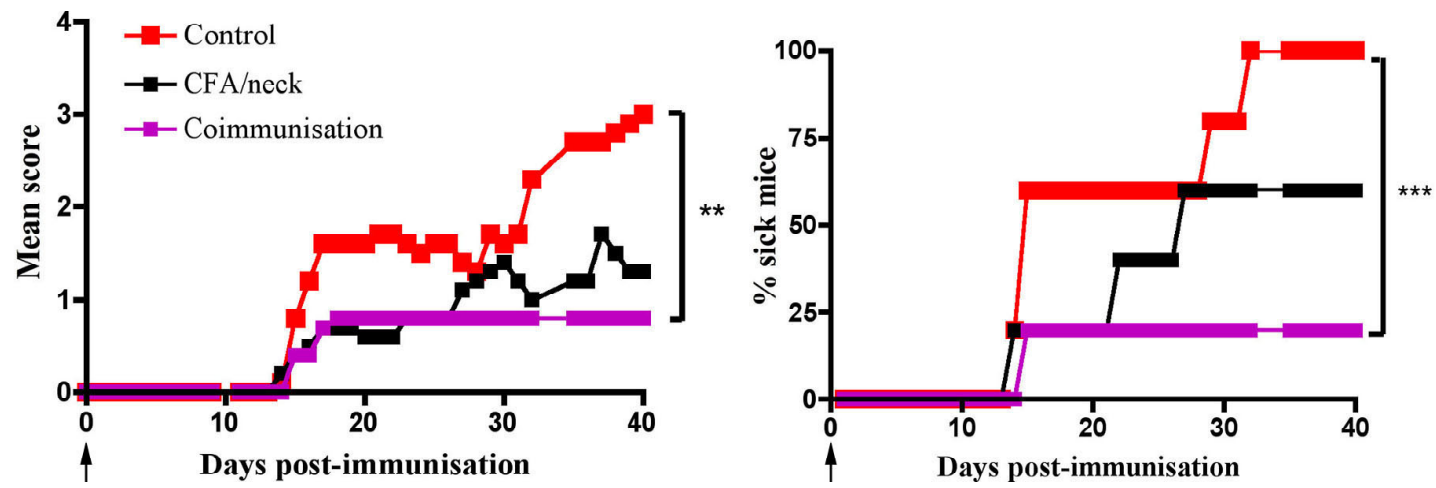

Figure 5.13 s.c. injection of $\mathrm{DM}^{-M} \mathrm{OG}_{35-55}$ in CFA on the neck protected mice against EAE

C57BL/6J were immunised with $2 \mu \mathrm{g}$ of $\mathrm{DM}-\mathrm{MOG}_{35-55}$ in CFA on the neck at day zero. As experimental control, mice were immunised with EAE inducing emulsion emulsified with (coimmunisation) or without 
(control) $2 \mu \mathrm{g}$ of $\mathrm{DM}-\mathrm{MOG}_{35-55}$. Left graph, mean EAE scores. Right graph, \%sick mice. Significant differences are indicated, $* * \mathrm{p}<0.01, * * * \mathrm{p}<0.001$. Analysis of statistical significance was performed using a one tailed Mann Whitney $U$ test. Each group contained five mice. DM-MOG $35-55 /$ IFA treatment as shown in Figure 5.14 was also included in this experiment. However, results are not indicated here for clarity.

To enhance therapeutic outcomes and reduce adverse effects of the DM-MOG $35-55$ treatment, DM-MOG $35-55$ was also tested in an IFA emulsion. Unlike CFA, IFA does not contain heat killed mycobacterium and was shown previously to perform better with $\mathrm{SM}_{-\mathrm{MOG}_{35-55} \text { for EAE suppression in mice }}{ }^{168}$. As shown in Figure 5.14, DM-MOG $35-55$ mixed in IFA also protected mice against EAE. Although it has been widely accepted that the heat-killed mycobacterium contained in CFA is an effective means of potentiating cellular response to the injected immunogens ${ }^{230}$, it did not affect the suppressive effect mediated by the DM-MOG $35-55$ treatment compared with IFA. The equal suppressive effect of CFA and IFA suggests that both of the adjuvants might work simply as slow release depot. In conclusion, s.c. injection of DM-MOG $35-55$ in CFA or IFA on the neck, which is remote from the site of EAE induction (at both flanks), protected mice against EAE.
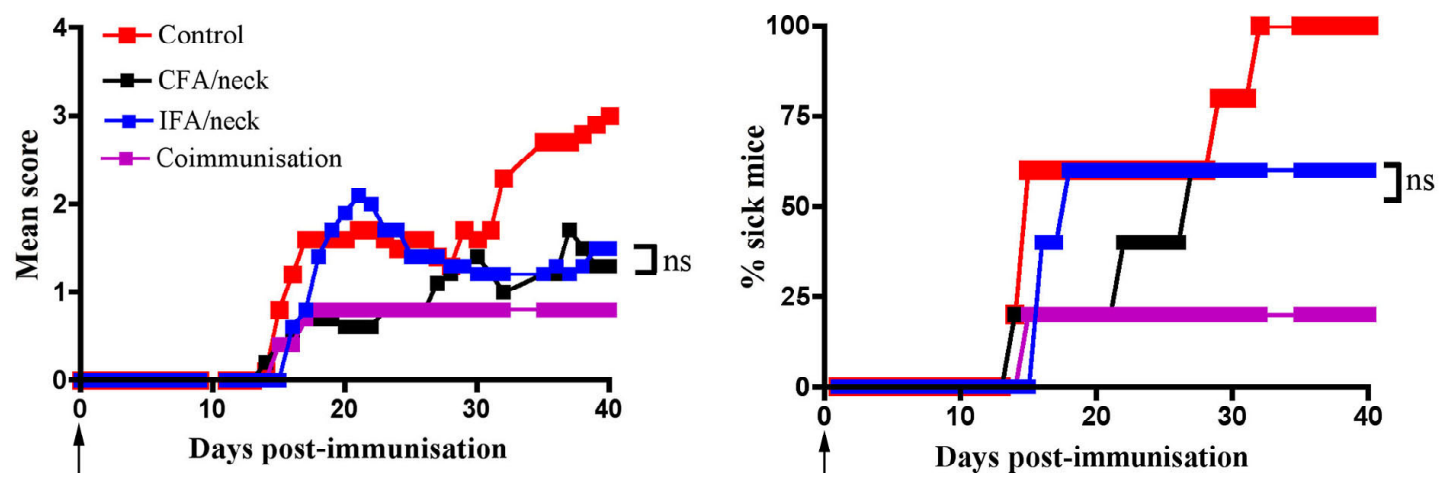

Figure 5.14 s.c. injection of ${\mathrm{DM}-M O G_{35-55}}$ in IFA on the neck protected mice against EAE

C57BL/6J were immunised with $2 \mu \mathrm{g}$ of $\mathrm{DM}^{-\mathrm{MOG}_{35-55}}$ in IFA on the neck at day zero. As experimental control, mice were immunised with EAE inducing emulsion mixed with (coimmunisation) or without (control) $2 \mu \mathrm{g}$ of $\mathrm{DM}-\mathrm{MOG}_{35-55}$. Left graph, mean EAE scores. Right graph, \%sick mice. Significant differences are indicated, ns, not significant. Analysis of statistical significance was performed using a one tailed Mann Whitney $U$ test. Each group contained five mice. Arrows indicate the days of $\mathrm{DM}-\mathrm{MOG}_{35-55}$ administration. 


\subsubsection{3 s.c. injection of $D^{D M-M O G}{ }_{35-55}$ in $P B S$ on the neck protected mice against} EAE

Although s.c. treatment with $\mathrm{DM}-\mathrm{MOG}_{35-55}$ in $\mathrm{CFA}$ or IFA protected the recipient mice against EAE, these preparations are not ideal for treating humans due to the side effects caused by Freund's adjuvant, such as pain and tissue damage. Therefore, I looked to develop a more clinically acceptable administration regime. Based on the idea that slow release may be required for $\mathrm{DM}-\mathrm{MOG}_{35-55}$ mediated EAE suppression, mice were treated on the neck with DM-MOG $35-55$ in PBS s.c. each day continuously for seven days before EAE induction. As shown in Figure 5.15, daily treatment with DM-MOG $35-55$ but not DM greatly suppressed EAE, supporting the proposal that continuous release is required for $\mathrm{DM}-\mathrm{MOG}_{35-55}$ mediated EAE suppression.
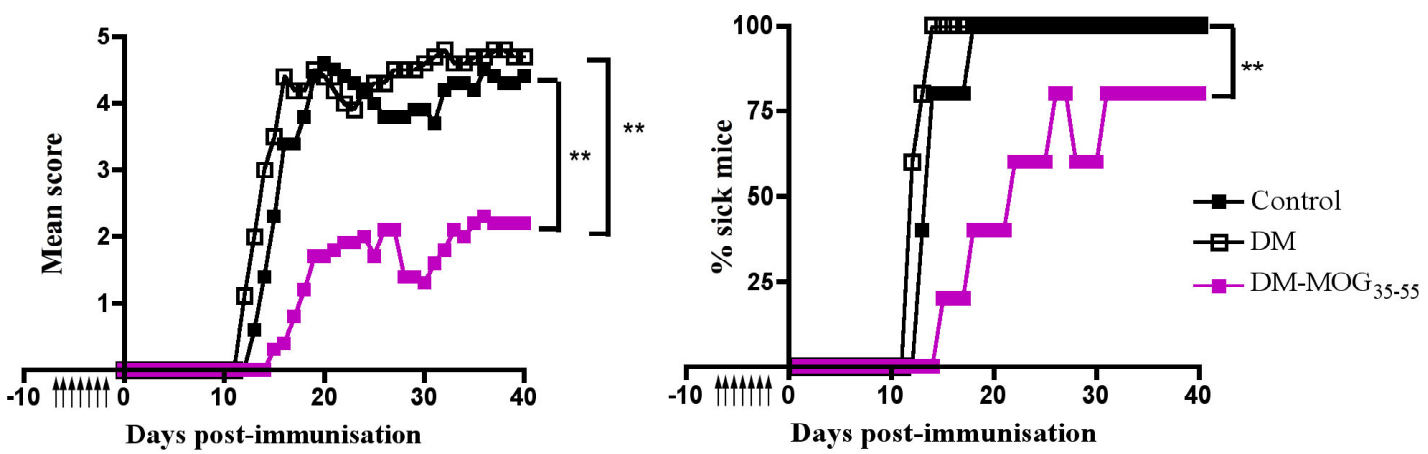

Figure 5.15 Multiple s.c. injection of DM-MOG ${ }_{35-55}$ in PBS suppressed EAE

Mice were treated with $1 \mu \mathrm{g}$ of DM or DM-MOG $35-55$ in PBS on the neck continuously for seven days before EAE induction (days -7 to -1 ). Mice treated with PBS on the neck continuously for seven days were used as the experimental controls. On day zero, EAE was induced in all the groups of mice. Left graph, mean clinical scores. Right graph, \%sick mice. Significant differences are indicated, ${ }^{* *} \mathrm{p}<0.01$. Analysis of statistical significance was performed using a one tailed Mann Whitney U test. Each group contained five mice. Arrows indicate the days of DM or DM-MOG $35-55$ injections.

\subsection{7 i.v. injection of DM did not suppress EAE}

As shown in Chapter 4, the binding of DM to bMDSCs greatly enhanced the bMDSC-dependent suppression of $\mathrm{T}$ cell proliferation in vitro (see Figure 4.22). Therefore, it was possible that the administration of DM could suppress EAE by enhancing bMDSC-dependent suppression of $\mathrm{T}$ cell proliferation in vivo.

To test the above hypothesis, up to $5 \mu \mathrm{g}$ of DM was i.v. injected into mice one day before EAE induction. However, as shown in Figure 5.16, the administration of DM i.v. did not protect mice against EAE. This lack of suppression may have been due to the 
dose of DM in the experiment. However, due to limited availability of DM, higher doses of DM could not be tested.
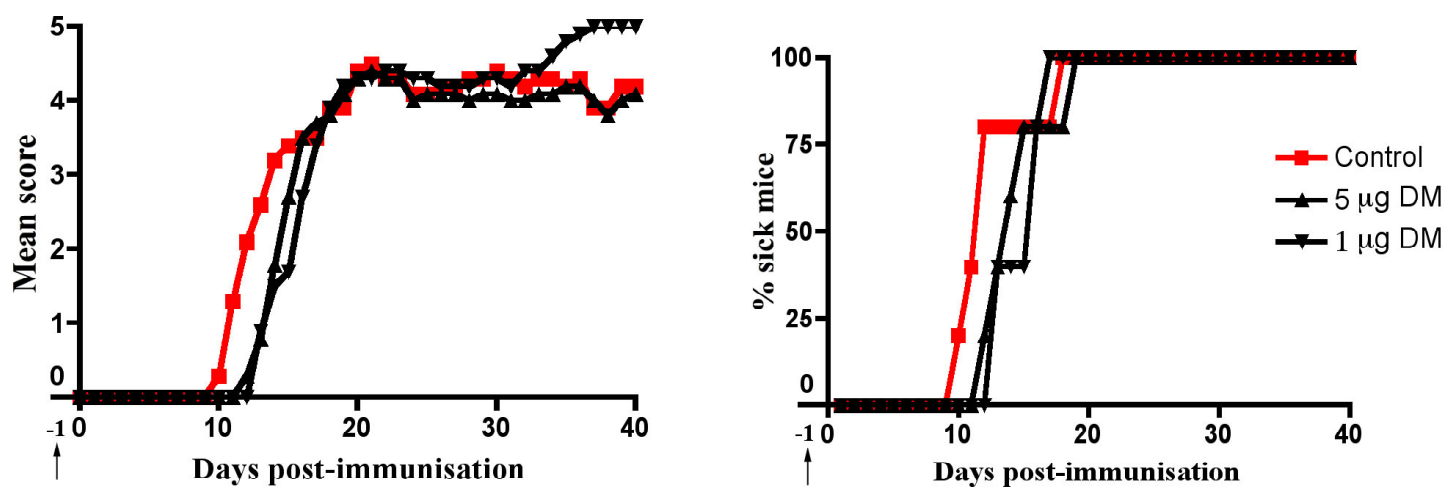

Figure 5.16 i.v. injection of DM did not suppress EAE

$\mathrm{DM}$ at the dose of $1 \mu \mathrm{g}$ or $5 \mu \mathrm{g}$ was injected into C57BL/6J mice one day before EAE induction. Ageand sex-matched mice were i.v. injected with $200 \mu \mathrm{l}$ PBS as the experimental controls. Left graph, mean clinical scores. Right graph, \%sick mice. Each group contained five mice. Arrows indicate the days for DM i.v. injection.

\subsection{Discussion}

The ideal treatments for MS and other autoimmune diseases are ones that target the disease-causing auto-antigen specific responses without compromising the ability of the immune system to respond to infections. However, most of the treatments for MS and other autoimmune diseases available today are antigen non-specific and have toxic side effects $^{41,114,120}$. In this chapter, I have shown that s.c. administration of DM-MOG $35-55$ resulted in effective suppression of EAE in mice via a unique $M_{30 G}$ s5 specific mechanism. This treatment has great potential to treat MS.

In Chapter 3, I showed that adding $\mathrm{SM}-\mathrm{MOG}_{35-55}$ to EAE inducing emulsion slightly reduced the disease scores of the recipient mice, and SM bound to bMDSCs in vivo when administered i.v.. This raised the possibility that bMDSCs played an important

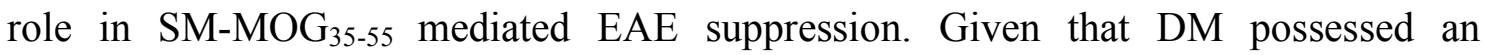
enhanced binding capability to bMDSCs compared to SM, I hypothesised that

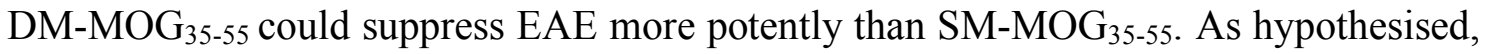
the experimental results showed that the addition of $\mathrm{DM}-\mathrm{MOG}_{35-55}$ to the EAE inducing emulsion greatly suppressed EAE in the recipient mice, and this suppression was even stronger than the one mediated by SM-MOG $35-55$. 
Importantly, DM-MOG $35-55$ treatment targeted the disease-causing $\mathrm{MOG}_{35-55}$ specific response and did not compromise the immune responses to other antigens. This conclusion was drawn from the following evidence. The DLNs isolated from mice treated with $\mathrm{DM}-\mathrm{MOG}_{35-55}$ showed an impaired response to $\mathrm{MOG}_{35-55}$ restimulation in vitro, and this impairment was particularly strong for the $\mathrm{DM}-\mathrm{MOG}_{35-55}$ treated mice that did not develop EAE. The impaired response was reflected by the reduced cell proliferation and cytokine production, i.e. the production of the two key cytokines for EAE, IL-17 and IFN- $\gamma^{137,144,146,148,149,231}$, were reduced. Importantly, T cell proliferation and cytokine production were not impaired when the DLN cells were restimulated in vitro with anti-CD3 antibody and PPD (the mycobacterium protein derivative contained in CFA), indicating that this suppression of $\mathrm{T}$ cell responses was $\mathrm{MOG}_{35-55}$ specific.

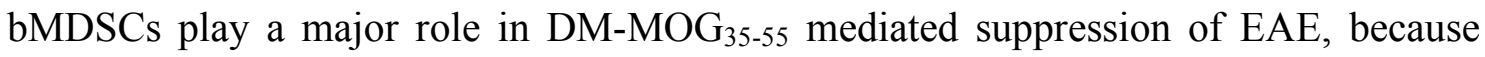
the bMDSCs from mice treated with $\mathrm{DM}^{-M_{0}} \mathrm{G}_{35-55}$ could transfer EAE suppression. Moreover, the bMDSCs from mice induced with EAE exhibited a reduced suppressive capability in vitro, and the $\mathrm{DM}-\mathrm{MOG}_{35-55}$ treatment restored the cells' in vitro suppression. Together, these results provide further evidence that bMDSCs play a key role in $\mathrm{DM}-\mathrm{MOG}_{35-55}$ mediated EAE suppression.

Although it is clear that bMDSCs are involved in $\mathrm{DM}-\mathrm{MOG}_{35-55}$ mediated EAE suppression, at this stage, the exact mechanisms involved are unclear. The following evidence indicates that besides bMDSC-medicated direct suppression of $\mathrm{T}$ cells, other suppressive mechanisms may be involved in $\mathrm{DM}-\mathrm{MOG}_{35-55}$ mediated EAE suppression. First, although the bMDSCs from naïve mice demonstrated similar suppressive

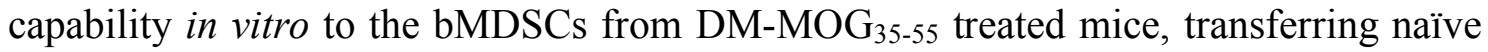
bMDSCs did not suppress EAE. The lack of EAE suppression following adoptive transfer of naïve bMDSCs indicates the involvement of additional suppressive mechanisms to the bMDSC-dependent direct $\mathrm{T}$ cell suppression alone. Second, DM-MOG $35-55$ but not DM suppressed EAE, indicating the involvement of MHCII presentation in the suppressive system. However, as shown in Chapter 4, most of the bMDSCs did not express MHCII on the surface at the time of isolation. The MHCII presentation involved in this suppression may be from host APCs. Alternatively, MHCII may be induced at later time points following DM-MOG $35-55$ exposure. Third, current experimental results could not exclude the involvement of other suppressor cells 
including Tregs. MDSCs have been reported to induce Tregs in vivo ${ }^{74}$ and thus, further studies are required to elucidate the involvement of Tregs in this system. The hypothesised mechanisms for $\mathrm{DM}-\mathrm{MOG}_{35-55}$ and bMDSCs mediated suppression in the EAE model are shown in Figure 5.17.

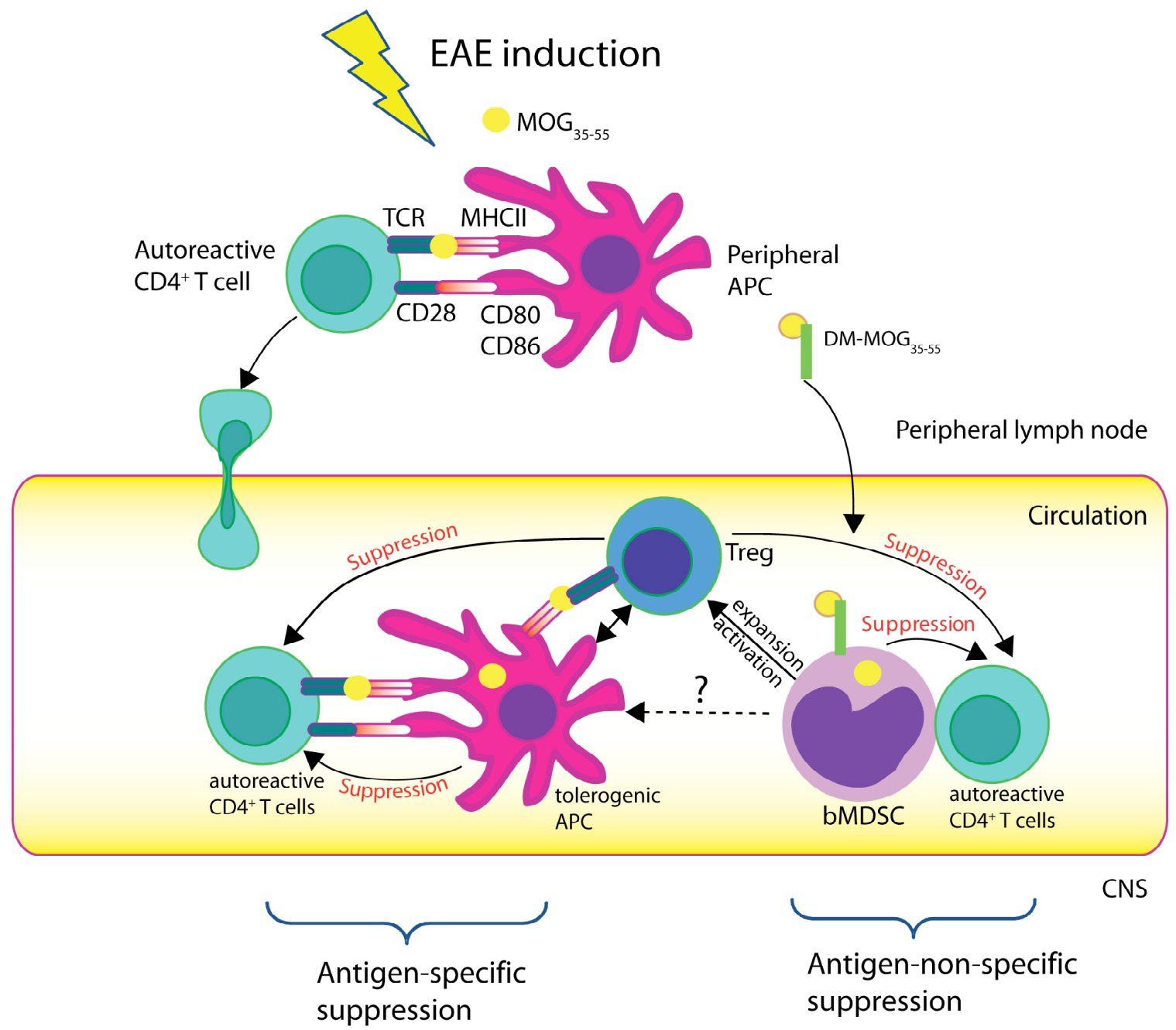

Figure 5.17 Hypothesised mechanisms for $\mathrm{DM}^{-M_{0}} \mathrm{G}_{35-55}$ and $\mathrm{bMDSCs}$ mediated suppression of EAE

When EAE is induced in mice, the APCs reside in DLNs processed and presented $\mathrm{MOG}_{35-55}$ to the $\mathrm{MOG}_{35-55}$ specific autoreactive $\mathrm{CD} 4^{+} \mathrm{T}$ cells. Once activated, these autoreactive $\mathrm{CD} 4^{+} \mathrm{T}$ cells migrate to the periphery and encounter the suppressive bMDSCs. There may be a few different mechanisms involved in bMDSCs mediated suppression of T cell responses in vivo. Firstly, bMDSCs could suppress $\mathrm{T}$ cells in an antigen-non-specific manner using NO as described in Chapter 4. The binding of DM to bMDSCs could enhance this suppressive effect. Secondly, the $\mathrm{MOG}_{35-55}$ peptide attached to DM is delivered to bMDSCs, and this may transform the bMDSCs, which are known as progenitors of APCs and have great plasticity, to tolerogenic APCs ${ }^{232}$. These tolerogenic APCs in turn suppress $\mathrm{T}$ cells via a $\mathrm{MOG}_{35-55}$ specific manner. This antigen-specific suppression could also be carried out directly or indirectly via the $\mathrm{MOG}_{35-55}$-specific Tregs. Alternatively, bMDSCs may transfer $\mathrm{MOG}_{35-55}$ to the host tolerogenic APCs, which could suppress $\mathrm{T}$ cells in a $\mathrm{MOG}_{35-55}$-specific manner. Thirdly, bMDSCs possibly activated or expanded Tregs, and these Tregs could suppress the autoreactive $\mathrm{CD} 4^{+} \mathrm{T}$ cells in either an antigen-specific or a non-specific manner. 
The location where EAE suppression takes place still remains to be identified. It has been reported that superantigens attract mononuclear cell infiltration ${ }^{233,234}$. Intramuscular injection of recombinant SEA, SEB and toxic shock syndrome toxin (TSST) induced marked mononuclear cell infiltration in muscle tissues ${ }^{233}$, and intracutaneous injection of SEB induced localised cutaneous inflammatory responses

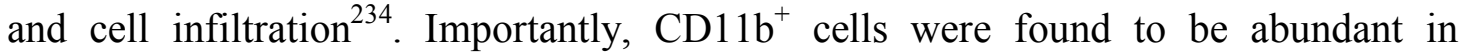
superantigen-induced inflammatory foci ${ }^{233}$. Although bMDSCs were never detected in the DLNs of the DM or DM-MOG $35-55$ injection sites, the possibility that bMDSCs were attracted to the tissues such as skin cannot be excluded. When mice were treated with $\mathrm{DM}_{-} \mathrm{MOG}_{35-55}$ at a remote site, the suppression of EAE was still effective, but not as strong as the suppression caused by coimmunisation. This suggests that both global and local suppression took place in the model. Further studies are required to elucidate this phenomenon.

In this chapter, I also attempted to develop a more clinically relevant therapy by

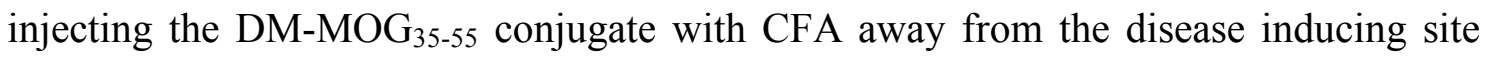
and the results were encouraging. However, due to the side effects of CFA such as pain and tissue damage, the $\mathrm{DM}-\mathrm{MOG}_{35-55} / \mathrm{CFA}$ formulation was considered unsuitable for human use $\mathrm{s}^{230}$. Therefore, I replaced CFA using IFA, which did not contain heat-killed mycobacterium. The treatment of $\mathrm{DM}-\mathrm{MOG}_{35-55} / \mathrm{IFA}$ also gave protection of EAE to the recipient mice. Both CFA and IFA provided similar levels of EAE suppression, suggesting that they might function as a slow release depot in this treatment regime. This possibility was supported by suppression of EAE following daily s.c. injection with $\mathrm{DM}-\mathrm{MOG}_{35-55}$ in PBS. Future studies are required to compare different adjuvant and drug carriers to achieve maximal suppression with minimum detrimental side effects. Furthermore, once this goal is achieved, the next step would be to test this therapy on mice at different stages of EAE.

In summary, in this chapter I demonstrated that the $\mathrm{DM}-\mathrm{MOG}_{35-55}$ treatment targeted the suppressor cell population, bMDSCs, and suppressed EAE in a $\mathrm{MOG}_{35-55}$ specific manner. This finding highlights the potential of the treatments targeting immunosuppressive cells to prevent autoimmune diseases or reduce their severity. In light of this study, there are still many questions to be answered in order to determine 


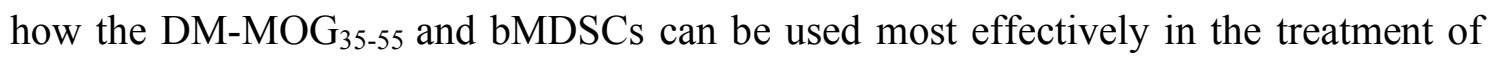
MS or other autoimmune diseases.

\subsection{Conclusions}

I have developed a treatment for EAE by using DM-MOG $35-55$. When DM-MOG $35-55$ was administered s.c. into the mice immunised with EAE, the disease was inhibited.

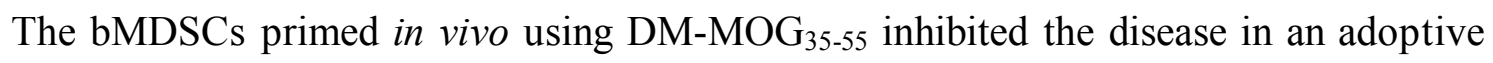
transfer model of EAE, indicating that bMDSCs play an important role in this EAE suppression. Moreover, this $\mathrm{DM}-\mathrm{MOG}_{35-55}$ treatment specifically targeted the $\mathrm{MOG}_{35-55}$ specific response but did not inhibit the immune responses to other stimuli. Thus, this antigen-specific therapy has great potential for the treatment of human MS. 


\section{Chapter 6:}

\section{General Discussion}




\subsection{Overview}

MS is an autoimmune disease of the CNS that causes a variety of symptoms including cognitive dysfunction, abnormal sensations, impairment of motor function, and severe paralysis $^{88,98}$. The ideal treatments for MS and other autoimmune diseases are ones that specifically target the disease-causing autoimmune responses without inhibiting the immune system's ability to respond to pathogens and infections. However, most of the treatments for MS and other autoimmune diseases available today are antigen non-specific ${ }^{41,100,120,130}$. Thus, this thesis was carried out aiming to develop an antigen-specific immunotherapy for EAE, with the ultimate goal of taking this research forward to develop a treatment for MS.

Based on the previous finding that the injection of SM-MOG $35-55 / \mathrm{IFA}$ s.c. into mice suppressed EAE in a $\mathrm{MOG}_{35-55}$-specific manner, and Tregs were the key players in this EAE suppression ${ }^{168}$, I hypothesised that the administration of SM-MOG $35-55 /$ IFA expanded and/or activated a population of $\mathrm{MOG}_{35-55}$-specific Tregs in vivo, which inhibited EAE. Thus, the overall aim of Chapter 3 was to test this hypothesis and understand the cellular and molecular changes during the SM-MOG $35-55 /$ IFA mediated EAE suppression in vivo.

Surprisingly, the experimental results from Chapter 3 showed that treating mice with

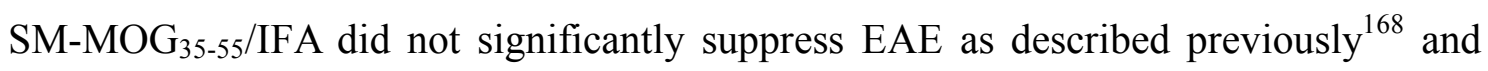
adoptive transfer of neither the Foxp $3^{+}$nor $\mathrm{CD}_{2} 5^{+}$Tregs from SM-MOG $35-55 / \mathrm{IFA}$ treated mice protected recipient mice against EAE. Although a large number of experiments have been carried out in our laboratory, the reason for the failure of EAE

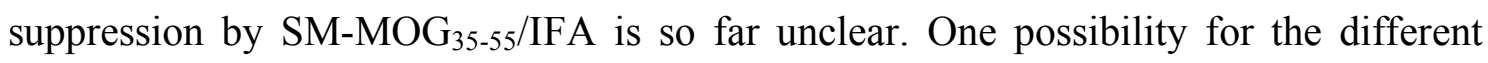
outcomes of this and the previous study lies in the difference in experimental conditions between the animal facilities where the initial study was done (at the University of Otago, Wellington School of Medicine and Health Sciences) compared to the institute's new facility (at Malaghan Institute of Medical Research). It has long been recognised that different animal facility environments often result in varying autoimmune disease susceptibility $^{133,235,236}$. In fact, the EAE incidence and severity observed in the current study was higher than that reported in the previous study, and the varying environmental conditions may have also played a role in the effectiveness of the SM-MOG $35-55 /$ IFA treatment. With greater EAE severity and incidence, it may be 
harder to induce an equivalent immune suppression response. The other possibility is the unknown differences between the SM in this study and the one prepared for the previous study (from the University of Auckland). Because the SM used in the previous study was no longer available, this possibility could not be tested.

Although a fully suppressive effect of $\mathrm{SM}-\mathrm{MOG}_{35-55} / \mathrm{IFA}$ treatment could not be repeated, the administration of $\mathrm{SM}-\mathrm{MOG}_{35-55}$ to mice using various methods repeatedly showed a minor suppressive effect on EAE, suggesting an in vivo suppressive feature of

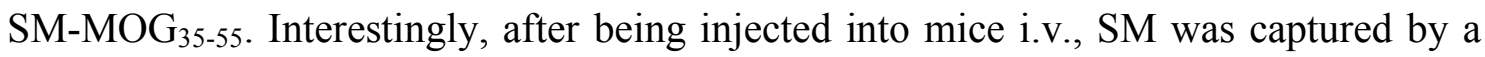
blood $\mathrm{MHCII} C \mathrm{CD} 11 \mathrm{~b}^{+} \mathrm{F} 4 / 80^{+} \mathrm{Gr}-1^{+}$cell population. Cells expressing the same surface markers have been reported to be suppressor cells called MDSCs in tumours ${ }^{45,75,76}$. This finding raised the possibility that the $\mathrm{SM}^{+} \mathrm{MHCII}{ }^{-} \mathrm{CD} 11 \mathrm{~b}^{+} \mathrm{F} 4 / 80^{+} \mathrm{Gr}-1^{+}$cells were suppressor cells e.g. a subpopulation of MDSCs, and were important in the minor suppression of EAE mediated by $\mathrm{SM}-\mathrm{MOG}_{35-55}$. This possibility was investigated in Chapter 4 and Chapter 5.

Because SM predominantly bound to $\mathrm{MHCII}^{+}$APCs and only a small proportion of SM bound to the blood $\mathrm{MHCII}^{-} \mathrm{CD} 11 \mathrm{~b}^{+} \mathrm{F} 4 / 80^{+} \mathrm{Gr}-1^{+}$cells, it was necessary to prevent SM-MHCII binding for the study of the blood MHCII'CD $11 \mathrm{~b}^{+} \mathrm{F} 4 / 80^{+} \mathrm{Gr}-1^{+}$cells using SM. To achieve this purpose, I used DM. In contrast to SM, DM is defective at both MHCII and TCR binding sites. It was hypothesised in Chapter 4 that in the absence of MHCII binding, DM would possess an enhanced binding capability to the blood $\mathrm{MHCII}^{-} \mathrm{CD} 11 \mathrm{~b}^{+} \mathrm{F} 4 / 80^{+} \mathrm{Gr}-1^{+}$cells, which were suppressor cells. The overall aim of Chapter 4 was to determine whether the $\mathrm{MHCII}^{-} \mathrm{CD} 11 \mathrm{~b}^{+} \mathrm{F} 4 / 80^{+} \mathrm{Gr}-1^{+}$blood cells were functional suppressor cells and to investigate the potential of DM to be used for targeted manipulation of these cells.

The experimental results from Chapter 4 showed that the blood $\mathrm{MHCII}^{-}$ $\mathrm{CD} 11 \mathrm{~b}^{+} \mathrm{F} 4 / 80^{+} \mathrm{Gr}-1^{+}$cells are potent suppressors of $\mathrm{T}$ cell responses in vitro, and thus, I named them the "bMDSCs". bMDSCs are different from MDSCs reported by others in three aspects. First, MDSCs are in the spleens, bone marrow and within primary and

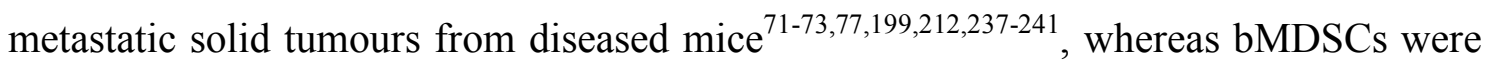
identified in the blood of naïve mice. Second, MDSCs are a heterogenous cell population consisting of neutrophilic cells and mononuclear cells ${ }^{45,71}$, whereas bMDSCs 
exclusively showed a monocytic morphology. Although one report demonstrated that the mononuclear cells within MDSC population (MO-MDSCs) are functional suppressor cells ${ }^{71}$, these MO-MDSCs are different from bMDSCs in various cell surface antigens. For example, MO-MDSCs express a high level of Ly6C and intermediate level of CD1d, whereas bMDSCs are heterogenous in Ly6C expression, ranging from negative to high, and do not have CD1d on their surface. Third, MDSCs suppress $\mathrm{T}$ cell proliferation in vitro using a variety of mechanisms, including up-regulation of NO production ${ }^{78,79}$ and arginase 1 induction $^{80-83,194}$, depending on what tumours they are derived from ${ }^{71}$. In contrast, bMDSCs suppress $\mathrm{T}$ cell response in a NOS-dependent but arginase-independent pathway. These results reveal that bMDSCs are a unique cell population different from MDSCs in tumour models. The existence of the bMDSC population in a natural disease-free condition suggests that these cells constitute a suppressive mechanism that has evolved to maintain immune homeostasis and limit uncontrolled immune responses.

Interestingly, the binding of DM to bMDSCs strongly enhanced the cells' suppressive activity in vitro. This enhancement has not been reported before and it highlighted the potential for utilising DM to develop an immunosuppressive treatment that targets bMDSCs. The enhanced suppressive activity of bMDSCs caused by the binding of mSAg raised the possibility that bMDSCs played a role in $\mathrm{SM}-\mathrm{MOG}_{35-55}$ mediated EAE suppression. This possibility and the therapeutic potential of DM for treating EAE were investigated in Chapter 5.

Because DM showed an enhanced binding over SM to the suppressor cells bMDSCs, and $\mathrm{SM}-\mathrm{MOG}_{35-55}$ suppressed EAE, in Chapter 5, I hypothesised that DM-MOG $35-55$ could suppress EAE, and bMDSCs played a key role in this EAE suppression. The overall aim of Chapter 5 was to test the above hypothesis and develop a successful immunotherapy for EAE as a potential treatment for MS. The experimental results from Chapter 5 showed that when $\mathrm{DM}^{-} \mathrm{MOG}_{35-55}$ was administered s.c. into the mice immunised with EAE, the disease was inhibited. Importantly, this DM-MOG $35-55$ treatment specifically targeted the $\mathrm{MOG}_{35-55}$ specific response but did not inhibit the immune responses to other stimuli. Thus, this antigen-specific therapy has great potential be be developed as the treatment for human MS. 
Currently there are six treatments available for MS, and three of them are fully subsidised by the New Zealand government (see Section 1.6.4) ${ }^{119}$. However, all of these treatments are antigen non-specific and their efficacy and side effect profile leaves ample room for improvement ${ }^{41}$. Although some antigen-specific therapies have been shown to successfully inhibit EAE, none of them have demonstrated clinical benefit for MS. For example, oral administration of MBP can suppress EAE in different species of $\operatorname{animals}^{242}$, however, the phase III trial using oral MBP did not show clinical benefit ${ }^{243}$. The other example is using the strategy called altered peptide ligands (APLs), the peptides that have an altered ability to bind to TCR or MHC, and result in T cell anergy in vivo. The MBP-derived APL has been shown to prevent and suppress the development of $\mathrm{EAE}^{244,245}$, but the development of MBP-derived APL for treatment of MS has been suspended owing to unexpected disease exacerbation and hypersensitivity reactions ${ }^{246,247}$. The $\mathrm{DM}^{-\mathrm{MOG}_{35-55}}$ treatment described in this thesis provides an alternative potential antigen-specific therapy for MS (see further discussion in Section 6.2). In addition, no obvious side effects were observed in the $\mathrm{DM}-\mathrm{MOG}_{35-55}$ treated mice.

Because adoptive transfer of the bMDSCs from $\mathrm{DM}-\mathrm{MOG}_{35-55}$ treated mice could transfer EAE suppression, bMDSCs are confirmed to be the key players in this

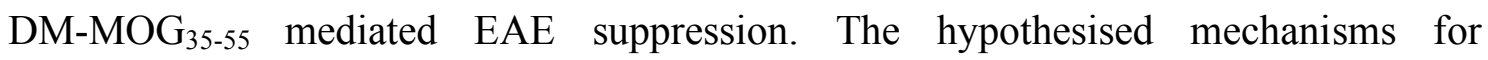
DM-MOG $35-55$ and bMDSCs mediated suppression of EAE are described in Figure 5.17. Although the exact mechanisms DM-MOG $35-55$ used to suppress EAE via bMDSCs are unclear, bMDSCs undoubtedly have great potential to be utilised in developing a therapy for the induction of antigen-specific suppression in patients with autoimmunity. This study provides evidence for the first time that bMDSCs could be used therapeutically to deactivate immune responses and induce immune tolerance in an animal model of autoimmune disease.

In summary, the results from this thesis underscore a previously unknown role of bMDSCs in limiting immune responses and indicate that harnessing bMDSCs in vivo could suppress autoimmune diseases. The findings of this thesis highlight the potential for targeting bMDSCs to develop an antigen-specific therapy for human MS and potentially other autoimmune diseases. 


\subsection{Clinical applications}

In this thesis, it was shown that the administration of $\mathrm{DM}-\mathrm{MOG}_{35-55}$ suppressed $\mathrm{MOG}_{35-55}$ induced EAE in a $\mathrm{MOG}_{35-55}$ specific manner. This DM-based antigen-specific treatment is certainly not solely restricted to the highly immunogenic $\mathrm{MOG}_{35-55}$ peptide. As in MS, the immune responses are not only against one, but a heterogenous group of CNS peptide antigens, the use of DM with a mixture of CNS peptides or proteins, may provide a broader and more biologically relevant effect. Moreover, this work also raised the possibility that the DM-autoantigen conjugates could also be applied and adapted to treat other autoimmune diseases.

The high affinity of DM to bind to bMDSCs and the internalisation of DM into the cells also raised a possibility that DM can be used to deplete bMDSCs by toxin delivery. For example, cytochrome c or diphtheria toxin could be conjugated with DM and delivered to bMDSCs to eliminate them in vivo. The application of DM-toxin conjugation has potential to be used as a therapeutic strategy to subvert the tumour-MDSC induced tolerogenic microenvironment and used in fundamental research on bMDSCs.

Moreover, DM-peptide or DM-protein can be used in combination with adjuvants to induce bMDSC differentiation into functional APCs. This could be an effective strategy for developing antigen-specific tumour therapies. The differentiation of bMDSCs will not only remove their suppressive effect, but also evoke an immune response against the antigen conjugated to DM. A variety of chemicals and proteins have been shown to alter myeloid cell or tumour MDSC differentiation and thus, have potential to be used as the adjuvants in this strategy. For example, the administration of all-trans-retinoic acid differentiated immature myeloid cells in vivo into mature DCs, macrophages and granulocytes $^{248}$, and the injection of IFN- $\gamma$ plus TNF- $\alpha$ altered MDSC differentiation into mature macrophages ${ }^{249}$.

In summary, with ongoing research into this field (see Section 6.3), it is expected that further advances towards effective clinical treatments for MS, other autoimmune diseases and cancer will be developed. 


\subsection{Implications for future research}

The work in this thesis conclusively demonstrated the involvement of bMDSCs in DM-MOG $35-55$-mediated suppression of EAE, but also raised questions on the precise mechanisms of action. The following two questions remain to be addressed. First, is the DM-MOG $35-55$ mediated suppression of $\mathrm{MOG}_{35-55}$ specific response caused by deletion or anergy of the $\mathrm{MOG}_{35-55}$ specific $\mathrm{T}$ cells? One potential solution is to investigate changes in the numbers, activation markers and apoptotic markers of the $\mathrm{MOG}_{35-55}$ specific $\mathrm{CD}^{+} \mathrm{T}$ cells after $\mathrm{DM}-\mathrm{MOG}_{35-55}$ treatment. However, it would be potentially difficult to develop the $\mathrm{MOG}_{35-55}$ tetramers for the detection of $\mathrm{MOG}_{35-55}$ specific $\mathrm{T}$ cells ex vivo. One way to overcome this problem is to use an adoptive transfer system in which CFSE labelled 2D2 CD45. $2^{+} \mathrm{CD} 4^{+} \mathrm{T}$ cells are transferred into CD45.1 congenic mice. After treating the recipient mice with the $\mathrm{DM}-\mathrm{MOG}_{35-55}$ treatment, the 2D2 $\mathrm{CD} 45.2^{+} \mathrm{CD}^{+} \mathrm{T}$ cells could be isolated at various time points and analysed for the above features. Second, are bMDSCs the only suppressor mechanism in the DM-MOG $35-55$ mediated EAE suppression? The direct way to answer this question is to deplete bMDSCs from mice prior to the $\mathrm{DM}-\mathrm{MOG}_{35-55}$ treatment. Although attempts were made to establish a method to deplete bMDSCs, unfortunately, none of them were feasible as these depletion methods also depleted or reduced the responses of other cell types important for EAE development. However, if this bMDSC depletion issue can be remedied, it would be of great interest to determine whether DM-MOG $35-55$ treatment also activate or induce other suppressor mechanisms in this EAE suppression.

It has not been determined by this work whether $\mathrm{DM}-\mathrm{MOG}_{35-55}$ treatment could induce bystander suppression, a process whereby the administration of one antigen can be used to prevent the development of disease with another antigen ${ }^{250}$. Bystander suppression is an important property because it would allow treatment of autoimmune diseases where the initiating antigen is unknown and where epitope spreading plays a pathological role. Epitope spreading refers to the process where an immune response to the epitope on a self-antigen triggers immune responses to neighbouring epitopes on the same antigen or other epitopes on nearby molecules ${ }^{251,252}$. However, the EAE model used in this thesis does not allow for the study of bystander suppression as the C57BL/6J mice are only susceptible to EAE induced by the $\mathrm{MOG}_{35-55}$ peptide $^{134}$. F1 crosses of mice or mice that are susceptible to the induction of EAE with more than one peptide could be used to study this mechanism. 
Furthermore, the EAE model used in this study is an induced disease, in that mice did not develop the disease spontaneously. It is therefore necessary to test this DM-autoantigen treatment in a spontaneous disease model system such as the autoimmune diabetes in non-obese diabetic (NOD) mice.

Further studies could be undertaken to determine the molecules involved in DM-bMDSC's binding and the mechanisms DM utilised to enhance bMDSCs' suppression. Because DM was taken into the cytoplasm of bMDSCs quickly after being injected into mice (within 20 minutes), it is likely that the DM uptake by bMDSCs is receptor-mediated. To characterise this receptor for DM, bMDSC lysate containing DM could be prepared, and a western blot analysis in combination with proteomics techniques could be applied to identify the protein to which DM binds. The identification of the receptor for DM would also help answer the question why DM did not bind to bMDSCs in vitro and understand the mechanisms utilised by DM to enhance the bMDSCs' in vitro suppressive activity.

It is also of interest, although beyond the scope of autoimmune diseases, whether tumour-MDSCs described by others, in particular, the MO-MDSCs ${ }^{71}$ are generated from bMDSCs. Further studies could be undertaken to determine the relationship between the tumour-MDSCs and bMDSCs. This may provide insight into the mechanisms tumours use to escape immune recognition.

Future studies are also required to compare different adjuvants and drug carriers to achieve maximal suppression with minimum detrimental side effect. Once this goal is achieved, the next step would be to test this therapy on mice at different stages of EAE. The long-term goal will be to improve the efficacy of the treatment without increasing the risks of side effects. This $\mathrm{DM}-\mathrm{MOG}_{35-55}$ treatment holds great promise to one day effectively treat MS and other autoimmune diseases with fewer side effects than current drugs. 


\section{References}


1. Kvell, K., Cooper, E.L., Engelmann, P., Bovari, J. \& Nemeth, P. Blurring borders: innate immunity with adaptive features. Clin Dev Immunol 2007, 83671 (2007).

2. Janeway, C.A., Jr. \& Medzhitov, R. Innate immune recognition. Annu Rev Immunol 20, 197-216 (2002).

3. Borghesi, L. \& Milcarek, C. Innate versus adaptive immunity: a paradigm past its prime? Cancer Res 67, 3989-3993 (2007).

4. Kabelitz, D. \& Medzhitov, R. Innate immunity--cross-talk with adaptive immunity through pattern recognition receptors and cytokines. Curr Opin Immunol 19, 1-3 (2007).

5. McDade, T.W. The Ecologies of Human Immune Function. Annual Review of Anthropology 34, 495-521 (2005).

6. Parish, C.R. Innate Immune Mechanisms: Nonself Recognition. Encyclopedia of Life Sciences Sep, 1-8 (2005).

7. Wong, F.S. \& Wen, L. Toll-Like Receptors and Diabetes. Annals of the New York Academy of Sciences 1150, 123-132 (2008).

8. Fischer, M. \& Ehlers, M. Toll-like Receptors in Autoimmunity. Annals of the New York Academy of Sciences 1143, 21-34 (2008).

9. Alberts, B., et al. Molecular Biology of the Cell, (Garland Science, New York and London, 2002).

10. Ahmed, R. \& Gray, D. Immunological memory and protective immunity: understanding their relation. Science 272, 54-60 (1996).

11. Stagg, A.J. \& Knight, S.C. Antigen-presenting Cells. Encyclopedia of Life Sciences, 1-8 (2001).

12. Nickoloff, B.J. \& Turka, L.A. Immunological functions of non-professional antigen-presenting cells: new insights from studies of T-cell interactions with keratinocytes. Immunol Today 15, 464-469 (1994).

13. Ferrero, I., Michelin, O. \& Luescher, I. Antigen Recognition by T Lymphocytes. Encyclopedia of Life Sciences, 1-10 (2007).

14. Stambas, J., et al. Killer T cells in influenza. Pharmacol Ther 120, 186-196 (2008).

15. Fouser, L.A., Wright, J.F., Dunussi-Joannopoulos, K. \& Collins, M. Th17 cytokines and their emerging roles in inflammation and autoimmunity. Immunological Reviews 226, 87-102 (2008). 
16. Ye Wang, D.C.M.J.A. The role of endoplasmic reticulum-associated protein degradation in MHC class I antigen processing. Immunological Reviews 172, 67-72 (1999).

17. Honey, K. \& Rudensky, A.Y. Lysosomal cysteine proteases regulate antigen presentation. Nat Rev Immunol 3, 472-482 (2003).

18. Chang, S.T., Ghosh, D., Kirschner, D.E. \& Linderman, J.J. Peptide length-based prediction of peptide-MHC class II binding. Bioinformatics 22, 2761-2767 (2006).

19. Nishio, J., Suzuki, M., Nanki, T., Miyasaka, N. \& Kohsaka, H. Development of TCRB CDR3 length repertoire of human T lymphocytes. Int Immunol 16, 423431 (2004).

20. Call, M.E., Pyrdol, J., Wiedmann, M. \& Wucherpfennig, K.W. The organizing principle in the formation of the $\mathrm{T}$ cell receptor-CD3 complex. Cell 111, 967979 (2002).

21. Garcia, K.C., et al. An alpha beta T Cell Receptor Structure at 2.5 A and Its Orientation in the TCR-MHC Complex. Science 274, 209-219 (1996).

22. Davis, M.M., et al. Ligand recognition by alpha beta T cell receptors. Annu Rev Immunol 16, 523-544 (1998).

23. Timmerman, L.A., Clipstone, N.A., Ho, S.N., Northrop, J.P. \& Crabtree, G.R. Rapid shuttling of NF-AT in discrimination of $\mathrm{Ca} 2+$ signals and immunosuppression. Nature 383, 837-840 (1996).

24. Lanzavecchia, A., Lezzi, G. \& Viola, A. From TCR engagement to T cell activation: a kinetic view of T cell behavior. Cell 96, 1-4 (1999).

25. van der Merwe, P.A. \& Davis, S.J. Molecular interactions mediating T cell antigen recognition. Annu Rev Immunol 21, 659-684 (2003).

26. Rudolph, M.G., Stanfield, R.L. \& Wilson, I.A. How TCRs bind MHCs, peptides, and coreceptors. Annu Rev Immunol 24, 419-466 (2006).

27. Konig, R. Interactions between MHC molecules and co-receptors of the TCR. Current Opinion in Immunology 14, 75-83 (2002).

28. Lenschow, D.J., Walunas, T.L. \& Bluestone, J.A. CD28/B7 system of T cell costimulation. Annu Rev Immunol 14, 233-258 (1996).

29. Freeman, G.J., et al. B7, a new member of the Ig superfamily with unique expression on activated and neoplastic B cells. J Immunol 143, 2714-2722 (1989). 
30. Lanier, L.L., et al. CD80 (B7) and CD86 (B70) provide similar costimulatory signals for T cell proliferation, cytokine production, and generation of CTL. $J$ Immunol 154, 97-105 (1995).

31. Linsley, P.S. \& Ledbetter, J.A. The role of the CD28 receptor during T cell responses to antigen. Annu Rev Immunol 11, 191-212 (1993).

32. Kyewski, B. \& Klein, L. A central role for central tolerance. Annu Rev Immunol 24, 571-606 (2006).

33. Starr, T.K., Jameson, S.C. \& Hogquist, K.A. Positive and negative selection of T cells. Annu Rev Immunol 21, 139-176 (2003).

34. Palmer, E. Negative selection--clearing out the bad apples from the T-cell repertoire. Nat Rev Immunol 3, 383-391 (2003).

35. Walker, L.S. \& Abbas, A.K. The enemy within: keeping self-reactive T cells at bay in the periphery. Nat Rev Immunol 2, 11-19 (2002).

36. Quill, H. Anergy as a mechanism of peripheral T cell tolerance. J Immunol 156, 1325-1327 (1996).

37. Schwartz, R.H. Models of $\mathrm{T}$ cell anergy: is there a common molecular mechanism? J Exp Med 184, 1-8 (1996).

38. Gaur, A., Wiers, B., Liu, A., Rothbard, J. \& Fathman, C.G. Amelioration of autoimmune encephalomyelitis by myelin basic protein synthetic peptideinduced anergy. Science 258, 1491-1494 (1992).

39. Zappia, E., et al. Mesenchymal stem cells ameliorate experimental autoimmune encephalomyelitis inducing T-cell anergy. Blood 106, 1755-1761 (2005).

40. Chatenoud, L. Anti-CD3 antibodies: towards clinical antigen-specific immunomodulation. Current Opinion in Pharmacology 4, 403-407 (2004).

41. Lopez-Diego, R.S. \& Weiner, H.L. Novel therapeutic strategies for multiple sclerosis [mdash] a multifaceted adversary. Nat Rev Drug Discov 7, 909-925 (2008).

42. Chang, T.T., Kuchroo, V.K. \& Sharpe, A.H. Role of the B7-CD28/CTLA-4 pathway in autoimmune disease. Curr Dir Autoimmun 5, 113-130 (2002).

43. Ishida, Y., Agata, Y., Shibahara, K. \& Honjo, T. Induced expression of PD-1, a novel member of the immunoglobulin gene superfamily, upon programmed cell death. EMBO J 11, 3887-3895 (1992). 
44. Sakaguchi, S., et al. Foxp3 $+\mathrm{CD} 25+\mathrm{CD} 4+$ natural regulatory $\mathrm{T}$ cells in dominant self-tolerance and autoimmune disease. Immunol Rev 212, 8-27 (2006).

45. Gabrilovich, D.I., et al. The Terminology Issue for Myeloid-Derived Suppressor Cells. Cancer Research 67, 425 (2007).

46. Fontenot, J.D. \& Rudensky, A.Y. A well adapted regulatory contrivance: regulatory $\mathrm{T}$ cell development and the forkhead family transcription factor Foxp3. Nat Immunol 6, 331-337 (2005).

47. Nishizuka, Y. \& Sakakura, T. Thymus and reproduction: sex-linked dysgenesia of the gonad after neonatal thymectomy in mice. Science 166, 753-755 (1969).

48. Jonuleit, H., et al. Identification and functional characterization of human $\mathrm{CD} 4(+) \mathrm{CD} 25(+) \mathrm{T}$ cells with regulatory properties isolated from peripheral blood. J Exp Med 193, 1285-1294 (2001).

49. Stephens, L.A., Mottet, C., Mason, D. \& Powrie, F. Human CD4(+)CD25(+) thymocytes and peripheral $\mathrm{T}$ cells have immune suppressive activity in vitro. Eur J Immunol 31, 1247-1254 (2001).

50. Kingsley, C.I., Karim, M., Bushell, A.R. \& Wood, K.J. CD25+CD4+ regulatory $\mathrm{T}$ cells prevent graft rejection: CTLA-4- and IL-10-dependent immunoregulation of alloresponses. J Immunol 168, 1080-1086 (2002).

51. Bruder, D., et al. Neuropilin-1: a surface marker of regulatory T cells. Eur $J$ Immunol 34, 623-630 (2004).

52. Asano, M., Toda, M., Sakaguchi, N. \& Sakaguchi, S. Autoimmune disease as a consequence of developmental abnormality of a $\mathrm{T}$ cell subpopulation. $J$ Exp Med 184, 387-396 (1996).

53. Allakhverdi, Z., et al. Expression of CD103 identifies human regulatory T-cell subsets. J Allergy Clin Immunol 118, 1342-1349 (2006).

54. Leithauser, F., et al. Foxp3-expressing CD103+ regulatory T cells accumulate in dendritic cell aggregates of the colonic mucosa in murine transfer colitis. Am J Pathol 168, 1898-1909 (2006).

55. Hori, S., Nomura, T. \& Sakaguchi, S. Control of regulatory T cell development by the transcription factor Foxp3. Science 299, 1057-1061 (2003).

56. Liu, W., et al. CD127 expression inversely correlates with FoxP3 and suppressive function of human CD4+ T reg cells. $J$ Exp Med 203, 1701-1711 (2006). 
57. Seddiki, N., et al. Expression of interleukin (IL)-2 and IL-7 receptors discriminates between human regulatory and activated T cells. $J$ Exp Med 203, 1693-1700 (2006).

58. Shimizu, J., Yamazaki, S., Takahashi, T., Ishida, Y. \& Sakaguchi, S. Stimulation of $\mathrm{CD} 25(+) \mathrm{CD} 4(+)$ regulatory $\mathrm{T}$ cells through GITR breaks immunological self-tolerance. Nat Immunol 3, 135-142 (2002).

59. Thornton, A.M. T regulatory cells. Curr Biol 15, R582 (2005).

60. Wing, K., et al. CTLA-4 control over Foxp3+ regulatory $\mathrm{T}$ cell function. Science 322, 271-275 (2008).

61. Cao, X., et al. Granzyme B and Perforin Are Important for Regulatory T CellMediated Suppression of Tumor Clearance. Immunity 27, 635-646 (2007).

62. Collison, L.W., et al. The inhibitory cytokine IL-35 contributes to regulatory Tcell function. Nature 450, 566-569 (2007).

63. Bluestone, J.A. \& Tang, Q. How do CD4+CD25+ regulatory T cells control autoimmunity? Curr Opin Immunol 17, 638-642 (2005).

64. Pandiyan, P., Zheng, L., Ishihara, S., Reed, J. \& Lenardo, M.J. $\mathrm{CD} 4+\mathrm{CD} 25+$ Foxp3+ regulatory $\mathrm{T}$ cells induce cytokine deprivation-mediated apoptosis of effector CD4+ T cells. Nat Immunol 8, 1353-1362 (2007).

65. Tang, Q., et al. Visualizing regulatory $\mathrm{T}$ cell control of autoimmune responses in nonobese diabetic mice. Nat Immunol 7, 83-92 (2006).

66. Onishi, Y., Fehervari, Z., Yamaguchi, T. \& Sakaguchi, S. Foxp3+ natural regulatory $\mathrm{T}$ cells preferentially form aggregates on dendritic cells in vitro and actively inhibit their maturation. Proc Natl Acad Sci U S A 105, 10113-10118 (2008).

67. Kryczek, I., et al. Cutting Edge: Induction of B7-H4 on APCs through IL-10: novel suppressive mode for regulatory T cells. J Immunol 177, 40-44 (2006).

68. Moseman, E.A., et al. Human plasmacytoid dendritic cells activated by CpG oligodeoxynucleotides induce the generation of $\mathrm{CD} 4+\mathrm{CD} 25+$ regulatory $\mathrm{T}$ cells. J Immunol 173, 4433-4442 (2004).

69. Cong, Y., et al. Generation of antigen-specific, Foxp3-expressing CD4+ regulatory $\mathrm{T}$ cells by inhibition of APC proteosome function. $J$ Immunol 174, 2787-2795 (2005).

70. Yamazaki, S., et al. Direct expansion of functional CD25+ CD4+ regulatory $\mathrm{T}$ cells by antigen-processing dendritic cells. $J$ Exp Med 198, 235-247 (2003). 
71. Movahedi, K., et al. Identification of discrete tumor-induced myeloid-derived suppressor cell subpopulations with distinct T cell-suppressive activity. Blood 111, 4233-4244 (2008).

72. Li, H., Han, Y., Guo, Q., Zhang, M. \& Cao, X. Cancer-expanded myeloidderived suppressor cells induce anergy of NK cells through membrane-bound TGF-beta1. J Immunol 182, 240-249 (2009).

73. Serafini, P., Mgebroff, S., Noonan, K. \& Borrello, I. Myeloid-derived suppressor cells promote cross-tolerance in B-cell lymphoma by expanding regulatory T cells. Cancer Res 68, 5439-5449 (2008).

74. Yang, R., et al. CD80 in immune suppression by mouse ovarian carcinomaassociated Gr-1+CD11b+ myeloid cells. Cancer Res 66, 6807-6815 (2006).

75. Krystal, G., et al. Re: the terminology issue for myeloid-derived suppressor cells. Cancer Res 67, 3986 (2007).

76. Yang, R. \& Roden, R.B.S. The Terminology Issue for Myeloid-Derived Suppressor Cells. Cancer Res 67, 426 (2007).

77. Ostrand-Rosenberg, S. \& Sinha, P. Myeloid-derived suppressor cells: linking inflammation and cancer. J Immunol 182, 4499-4506 (2009).

78. Mazzoni, A., et al. Myeloid suppressor lines inhibit T cell responses by an NOdependent mechanism. J Immunol 168, 689-695 (2002).

79. Bingisser, R.M., Tilbrook, P.A., Holt, P.G. \& Kees, U.R. Macrophage-derived nitric oxide regulates $\mathrm{T}$ cell activation via reversible disruption of the Jak3/STAT5 signaling pathway. J Immunol 160, 5729-5734 (1998).

80. Rodriguez, P.C., et al. Arginase I production in the tumor microenvironment by mature myeloid cells inhibits $\mathrm{T}$-cell receptor expression and antigen-specific Tcell responses. Cancer Res 64, 5839-5849 (2004).

81. Rodriguez, P.C., Quiceno, D.G. \& Ochoa, A.C. L-arginine availability regulates T-lymphocyte cell-cycle progression. Blood 109, 1568-1573 (2007).

82. Rodriguez, P.C. \& Ochoa, A.C. Arginine regulation by myeloid derived suppressor cells and tolerance in cancer: mechanisms and therapeutic perspectives. Immunol Rev 222, 180-191 (2008).

83. Rodriguez, P.C., et al. Arginase I in myeloid suppressor cells is induced by COX-2 in lung carcinoma. J Exp Med 202, 931-939 (2005).

84. Zhou, R., et al. Myeloid suppressor cell-associated immune dysfunction in CSA1M fibrosarcoma tumor-bearing mice. Cancer Sci 98, 882-889 (2007). 
85. Kusmartsev, S., Nefedova, Y., Yoder, D. \& Gabrilovich, D.I. Antigen-specific inhibition of CD8 $+\mathrm{T}$ cell response by immature myeloid cells in cancer is mediated by reactive oxygen species. J Immunol 172, 989-999 (2004).

86. Brys, L., et al. Reactive Oxygen Species and 12/15-Lipoxygenase Contribute to the Antiproliferative Capacity of Alternatively Activated Myeloid Cells Elicited during Helminth Infection. J Immunol 174, 6095-6104 (2005).

87. Sinha, P., Clements, V.K. \& Ostrand-Rosenberg, S. Reduction of MyeloidDerived Suppressor Cells and Induction of M1 Macrophages Facilitate the Rejection of Established Metastatic Disease. J Immunol 174, 636-645 (2005).

88. Lutton, J.D., Winston, R. \& Rodman, T.C. Multiple sclerosis: etiological mechanisms and future directions. Exp Biol Med (Maywood) 229, 12-20 (2004).

89. Volpé, R. Autoimmune Disease. Encyclopedia of Life Sciences 2001, 1-7 (2001).

90. Munz, C., Lunemann, J.D., Getts, M.T. \& Miller, S.D. Antiviral immune responses: triggers of or triggered by autoimmunity? Nat Rev Immunol 9, 246258 (2009).

91. Dyment, D.A., Sadovnick, A.D. \& Ebers, G.C. Genetics of multiple sclerosis. Hum Mol Genet 6, 1693-1698 (1997).

92. Gregersen, P.K. \& Olsson, L.M. Recent Advances in the Genetics of Autoimmune Disease. Annual Review of Immunology 27, 363-391 (2009).

93. Fujinami, R.S. \& Oldstone, M.B. Amino acid homology between the encephalitogenic site of myelin basic protein and virus: mechanism for autoimmunity. Science 230, 1043-1045 (1985).

94. Wucherpfennig, K.W. Mechanisms for the induction of autoimmunity by infectious agents. J Clin Invest 108, 1097-1104 (2001).

95. Olson, J.K., Ludovic Croxford, J. \& Miller, S.D. Innate and adaptive immune requirements for induction of autoimmune demyelinating disease by molecular mimicry. Mol Immunol 40, 1103-1108 (2004).

96. Gutcher, I. \& Becher, B. APC-derived cytokines and T cell polarization in autoimmune inflammation. J Clin Invest 117, 1119-1127 (2007).

97. El Behi, M., et al. New insights into cell responses involved in experimental autoimmune encephalomyelitis and multiple sclerosis. Immunol Lett 96, 11-26 (2005). 
98. Sospedra, M. \& Martin, R. Immunology of multiple sclerosis. Annu Rev Immunol 23, 683-747 (2005).

99. auckland, T.m.s.s.o. The multiple sclerosis society of auckland. (2006).

100. Vollmer, T.L., Preiningerova, J. \& Waxman, S.G. Multiple sclerosis. Encyclopedia of Life Sciences, 1-7 (2002).

101. Ebers, G.C., et al. A population-based study of multiple sclerosis in twins. $N$ Engl J Med 315, 1638-1642 (1986).

102. Dyment, D.A., et al. A multigenerational family with multiple sclerosis. Brain 125, 1474-1482 (2002).

103. Dyment, D.A., et al. Complex interactions among MHC haplotypes in multiple sclerosis: susceptibility and resistance. Hum Mol Genet 14, 2019-2026 (2005).

104. Norman, J.E., Jr., Kurtzke, J.F. \& Beebe, G.W. Epidemiology of multiple sclerosis in U.S. veterans: 2. Latitude, climate and the risk of multiple sclerosis. J Chronic Dis 36, 551-559 (1983).

105. Gold, S.M. \& Voskuhl, R.R. Estrogen treatment in multiple sclerosis. Journal of the Neurological Sciences In Press, Corrected Proof(2009).

106. Goebels, N., et al. Repertoire dynamics of autoreactive T cells in multiple sclerosis patients and healthy subjects: epitope spreading versus clonal persistence. Brain 123 Pt 3, 508-518 (2000).

107. Elisabetta, P., et al. HLA-DRB5*0101 and -DRB1*1501 expression in the multiple sclerosis-associated HLA-DR15 haplotype. Journal of Neuroimmunology 167, 108-119 (2005).

108. Krogsgaard, M., et al. Visualization of myelin basic protein (MBP) T cell epitopes in multiple sclerosis lesions using a monoclonal antibody specific for the human histocompatibility leukocyte antigen (HLA)-DR2-MBP 85-99 complex. J Exp Med 191, 1395-1412 (2000).

109. Oksenberg, J.R., et al. Selection for T-cell receptor V beta-D beta-J beta gene rearrangements with specificity for a myelin basic protein peptide in brain lesions of multiple sclerosis. Nature 362, 68-70 (1993).

110. Marta, M., Meier, U.C. \& Lobell, A. Regulation of autoimmune encephalomyelitis by toll-like receptors. Autoimmun Rev (2009).

111. Nagelkerken, L. Role of Th1 and Th2 cells in autoimmune demyelinating disease. Braz J Med Biol Res 31, 55-60 (1998). 
112. Gold, R., Linington, C. \& Lassmann, H. Understanding pathogenesis and therapy of multiple sclerosis via animal models: 70 years of merits and culprits in experimental autoimmune encephalomyelitis research. Brain 129, 1953-1971 (2006).

113. Sriram, S. \& Steiner, I. Experimental allergic encephalomyelitis: a misleading model of multiple sclerosis. Ann Neurol 58, 939-945 (2005).

114. Gold, R. Combination therapies in multiple sclerosis. Journal of Neurology 255, 51-60 (2008).

115. Tejada-Simon, M.V., Zang, Y.C., Hong, J., Rivera, V.M. \& Zhang, J.Z. Crossreactivity with myelin basic protein and human herpesvirus-6 in multiple sclerosis. Ann Neurol 53, 189-197 (2003).

116. Viglietta, V., Baecher-Allan, C., Weiner, H.L. \& Hafler, D.A. Loss of functional suppression by $\mathrm{CD} 4+\mathrm{CD} 25+$ regulatory $\mathrm{T}$ cells in patients with multiple sclerosis. J Exp Med 199, 971-979 (2004).

117. Honey, K. Reduction in regulation. Nat Rev Immunol 4, 322-322 (2004).

118. Sauter, E. \& Ono, M. A Potential New MS Treatment's Long and Winding Road. News and Views 9(2009).

119. Living with MS. Vol. 2009 (the Multiple Sclerosis Society of New Zealand, 2009).

120. Lam, S., Wang, S. \& Gottesman, M. Interferon-beta1b for the treatment of multiple sclerosis. Expert Opin Drug Metab Toxicol 4, 1111-1117 (2008).

121. Arnon, R. The development of Cop 1 (Copaxone), an innovative drug for the treatment of multiple sclerosis: personal reflections. Immunol Lett 50, 1-15 (1996).

122. Duda, P.W., Schmied, M.C., Cook, S.L., Krieger, J.I. \& Hafler, D.A. Glatiramer acetate (Copaxone) induces degenerate, Th2-polarized immune responses in patients with multiple sclerosis. J Clin Invest 105, 967-976 (2000).

123. Neuhaus, O., et al. Multiple sclerosis: comparison of copolymer-1- reactive T cell lines from treated and untreated subjects reveals cytokine shift from $\mathrm{T}$ helper 1 to T helper 2 cells. Proc Natl Acad Sci U S A 97, $7452-7457$ (2000).

124. Aharoni, R., Teitelbaum, D., Sela, M. \& Arnon, R. Copolymer 1 induces T cells of the $\mathrm{T}$ helper type 2 that crossreact with myelin basic protein and suppress experimental autoimmune encephalomyelitis. Proc Natl Acad Sci U S 94, 10821-10826 (1997). 
125. Aharoni, R., Teitelbaum, D., Arnon, R. \& Sela, M. Copolymer 1 acts against the immunodominant epitope $82-100$ of myelin basic protein by $\mathrm{T}$ cell receptor antagonism in addition to major histocompatibility complex blocking. Proc Natl Acad Sci U S A 96, 634-639 (1999).

126. Fridkis-Hareli, M., et al. Direct binding of myelin basic protein and synthetic copolymer 1 to class II major histocompatibility complex molecules on living antigen-presenting cells--specificity and promiscuity. Proc Natl Acad Sci U S A 91, 4872-4876 (1994).

127. Hong, J., Li, N., Zhang, X., Zheng, B. \& Zhang, J.Z. Induction of CD4+CD25+ regulatory $\mathrm{T}$ cells by copolymer-I through activation of transcription factor Foxp3. Proc Natl Acad Sci U S A 102, 6449-6454 (2005).

128. Jee, Y., et al. $\mathrm{CD} 4(+) \mathrm{CD} 25(+)$ regulatory $\mathrm{T}$ cells contribute to the therapeutic effects of glatiramer acetate in experimental autoimmune encephalomyelitis. Clin Immunol 125, 34-42 (2007).

129. Aharoni, R., et al. Demyelination arrest and remyelination induced by glatiramer acetate treatment of experimental autoimmune encephalomyelitis. Proc Natl Acad Sci U S A 105, 11358-11363 (2008).

130. Diem, R., Sattler, M.B. \& Bahr, M. Neurodegeneration and -protection in autoimmune CNS inflammation. J Neuroimmunol (2006).

131. Taneja, V. \& David, C.S. Lessons from animal models for human autoimmune diseases. Nat Immunol 2, 781-784 (2001).

132. Baxter, A.G. The origin and application of experimental autoimmune encephalomyelitis. Nat Rev Immunol 7, 904 (2007).

133. Racke, M.K. Experimental autoimmune encephalomyelitis (EAE). Curr Protoc Neurosci Chapter 9, Unit9 7 (2001).

134. Itzhack Mendel, N.K.d.R.A.B.-N. A myelin oligodendrocyte glycoprotein peptide induces typical chronic experimental autoimmune encephalomyelitis in $\mathrm{H}-2 \mathrm{~b}$ mice: Fine specificity and $\mathrm{T}$ cell receptor Vbeta expression of encephalitogenic T cells. European Journal of Immunology 25, 1951-1959 (1995).

135. Bernard, C.C., Leydon, J. \& Mackay, I.R. T cell necessity in the pathogenesis of experimental autoimmune encephalomyelitis in mice. Eur J Immunol 6, 655-660 (1976). 
136. Stromnes, I.M., Cerretti, L.M., Liggitt, D., Harris, R.A. \& Goverman, J.M. Differential regulation of central nervous system autoimmunity by $\mathrm{T}(\mathrm{H}) 1$ and T(H)17 cells. Nat Med 14, 337-342 (2008).

137. Aranami, T. \& Yamamura, T. Th17 Cells and autoimmune encephalomyelitis (EAE/MS). Allergol Int 57, 115-120 (2008).

138. Matthaei, K.I., Foster, P. \& Young, I.G. The role of interleukin-5 (IL-5) in vivo: studies with IL-5 deficient mice. Mem Inst Oswaldo Cruz 92 Suppl 2, 63-68 (1997).

139. Sanderson, C.J. Interleukin-5, eosinophils, and disease. Blood 79, 3101-3109 (1992).

140. Sabatino, J.J., Jr., Shires, J., Altman, J.D., Ford, M.L. \& Evavold, B.D. Loss of IFN-gamma enables the expansion of autoreactive CD4+ $\mathrm{T}$ cells to induce experimental autoimmune encephalomyelitis by a nonencephalitogenic myelin variant antigen. J Immunol 180, 4451-4457 (2008).

141. Afanasyeva, M., et al. Interleukin-12 receptor/STAT4 signaling is required for the development of autoimmune myocarditis in mice by an interferon-gammaindependent pathway. Circulation 104, 3145-3151 (2001).

142. Ivanov, II, et al. The orphan nuclear receptor RORgammat directs the differentiation program of proinflammatory IL-17+ T helper cells. Cell 126, 1121-1133 (2006).

143. Weaver, C.T., Harrington, L.E., Mangan, P.R., Gavrieli, M. \& Murphy, K.M. Th17: An Effector CD4 T Cell Lineage with Regulatory T Cell Ties. Immunity 24, 677-688 (2006).

144. Komiyama, Y., et al. IL-17 plays an important role in the development of experimental autoimmune encephalomyelitis. J Immunol 177, 566-573 (2006).

145. Chen, Y., et al. Anti-IL-23 therapy inhibits multiple inflammatory pathways and ameliorates autoimmune encephalomyelitis. J Clin Invest 116, 1317-1326 (2006).

146. Langrish, C.L., et al. IL-23 drives a pathogenic $\mathrm{T}$ cell population that induces autoimmune inflammation. J Exp Med 201, 233-240 (2005).

147. O'Connor, R.A., et al. Cutting edge: Th1 cells facilitate the entry of Th17 cells to the central nervous system during experimental autoimmune encephalomyelitis. J Immunol 181, 3750-3754 (2008). 
148. Tato, C.M. \& O'Shea, J.J. Immunology: What does it mean to be just 17? Nature 441, 166 (2006).

149. Furuzawa-Carballeda, J., Vargas-Rojas, M.I. \& Cabral, A.R. Autoimmune inflammation from the Th17 perspective. Autoimmunity Reviews 6, 169-175 (2007).

150. Veldhoen, M., Hocking, R.J., Flavell, R.A. \& Stockinger, B. Signals mediated by transforming growth factor-beta initiate autoimmune encephalomyelitis, but chronic inflammation is needed to sustain disease. Nat Immunol 7, 1151-1156 (2006).

151. Fossiez, F., et al. Interleukin-17. Int Rev Immunol 16, 541-551 (1998).

152. Bettelli, E., et al. Reciprocal developmental pathways for the generation of pathogenic effector TH17 and regulatory T cells. Nature 441, 235 (2006).

153. Veldhoen, M., Hocking, R.J., Atkins, C.J., Locksley, R.M. \& Stockinger, B. TGF $\beta$ in the context of an inflammatory cytokine milieu supports de novo differentiation of IL-17-producing T cells. Immunity 24, 179-189 (2006).

154. Mangan, P.R., et al. Transforming growth factor- $\beta$ induces development of the TH17 lineage. Nature 441, 231 (2006).

155. Cua, D.J., et al. Interleukin-23 rather than interleukin-12 is the critical cytokine for autoimmune inflammation of the brain. Nature 421, 744-748 (2003).

156. Harris, T.J., et al. Cutting edge: An in vivo requirement for STAT3 signaling in TH17 development and TH17-dependent autoimmunity. J Immunol 179, 43134317 (2007).

157. Yang, X.O., et al. STAT3 regulates cytokine-mediated generation of inflammatory helper T cells. J Biol Chem 282, 9358-9363 (2007).

158. McGeachy, M.J., et al. TGF-beta and IL-6 drive the production of IL-17 and IL10 by $\mathrm{T}$ cells and restrain $\mathrm{T}(\mathrm{H})-17$ cell-mediated pathology. Nat Immunol 8, 1390-1397 (2007).

159. Bettelli, E., Oukka, M. \& Kuchroo, V.K. T(H)-17 cells in the circle of immunity and autoimmunity. Nat Immunol 8, 345-350 (2007).

160. Kohm, A.P., Carpentier, P.A., Anger, H.A. \& Miller, S.D. Cutting edge: $\mathrm{CD} 4+\mathrm{CD} 25+$ regulatory $\mathrm{T}$ cells suppress antigen-specific autoreactive immune responses and central nervous system inflammation during active experimental autoimmune encephalomyelitis. J Immunol 169, 4712-4716 (2002). 
161. Gartner, D., Hoff, H., Gimsa, U., Burmester, G.-R. \& Brunner-Weinzierl, M.C. CD25 regulatory $\mathrm{T}$ cells determine secondary but not primary remission in EAE: Impact on long-term disease progression. Journal of Neuroimmunology 172, 73 (2006).

162. McNeill, A., Spittle, E. \& Backstrom, B.T. Partial depletion of CD69lowexpressing natural regulatory $\mathrm{T}$ cells with the anti-CD25 monoclonal antibody PC61. Scand J Immunol 65, 63-69 (2007).

163. Tarbell, K.V., Yamazaki, S., Olson, K., Toy, P. \& Steinman, R.M. CD25+ CD4+ T cells, expanded with dendritic cells presenting a single autoantigenic peptide, suppress autoimmune diabetes. J Exp Med 199, 1467-1477 (2004).

164. Tang, Q., et al. In vitro-expanded antigen-specific regulatory $\mathrm{T}$ cells suppress autoimmune diabetes. $J$ Exp Med 199, 1455-1465 (2004).

165. Ochoa-Reparaz, J., et al. Regulatory $\mathrm{T}$ cell vaccination without autoantigen protects against experimental autoimmune encephalomyelitis. J Immunol 178, 1791-1799 (2007).

166. Tischner, D., et al. Polyclonal expansion of regulatory $\mathrm{T}$ cells interferes with effector cell migration in a model of multiple sclerosis. Brain (2006).

167. Chen, X., Oppenheim, J.J., Winkler-Pickett, R.T., Ortaldo, J.R. \& Howard, O.M. Glucocorticoid amplifies IL-2-dependent expansion of functional FoxP3(+)CD4(+)CD25(+) T regulatory cells in vivo and enhances their capacity to suppress EAE. Eur J Immunol 36, 2139-2149 (2006).

168. McNeill, A.L. PhD Thesis: Suppression of experimental autoimmune encephalomyelitis by myelin-specific regulatory $\mathrm{T}$ cells. University of Otago, New Zealand (2005).

169. Zhu, B., et al. CD11b+Ly-6C(hi) suppressive monocytes in experimental autoimmune encephalomyelitis. J Immunol 179, 5228-5237 (2007).

170. Nicholson, L.B., Raveney, B.J. \& Munder, M. Monocyte dependent regulation of autoimmune inflammation. Curr Mol Med 9, 23-29 (2009).

171. Proft, T., et al. The Streptococcal superantigen SMEZ exhibits wide allelic variation, mosaic structure, and significant antigenic variation. J. Exp. Med. 191, 1765-1776 (2000).

172. Renno, T. Superantigens. Encyclopedia of Life Sciences, 1-5 (2001).

173. Arcus, V.L., et al. Conservation and variation in superantigen structure and activity highlighted by the three-dimensional structures of two new 
superantigens from Streptococcus pyogenes. Journal of Molecular Biology 299, 157 (2000).

174. Kamezawa, Y., et al. Streptococcal mitogenic exotoxin Z, a novel acidic superantigenic toxin produced by a T1 strain of Streptococcus pyogenes. Infect. Immun. 65, 3828-3833 (1997).

175. Proft, T., Louise Moffatt, S., Berkahn, C.J. \& Fraser, J.D. Identification and characterization of novel superantigens from Streptococcus pyogenes. J. Exp. Med. 189, 89-102 (1999).

176. Renno, T. \& Acha-Orbea, H. Superantigens in autoimmune diseases: still more shades of gray. Immunol Rev 154, 175-191 (1996).

177. Schiffenbauer, J., Johnson, H.M., Butfiloski, E.J., Wegrzyn, L. \& Soos, J.M. Staphylococcal enterotoxins can reactivate experimental allergic encephalomyelitis. Proceedings of the National Academy of Science, USA 90, 8543-8546 (1993).

178. Schiffenbauer, J., Soos, J. \& Johnson, H. The possible role of bacterial superantigens in the pathogenesis of autoimmune disorders. Immunol Today 19, 117-120 (1998).

179. Proft, T., et al. The streptococcal superantigen SMEZ exhibits wide allelic variation, mosaic structure, and significant antigenic variation. Journal of experimental medicine 191, 1765-1776 (2000).

180. Miller, C., Ragheb, J.A. \& Schwartz, R.H. Anergy and Cytokine-mediated Suppression as distinct superantigen-induced tolerance mechanisms in vivo. Journal of Experimental Medicine 190, 53-64 (1999).

181. Grundstrom, S., Cederbom, L., Sundstedt, A., Schiepers, P. \& Ivars, F. Superantigen-induced regulatory $\mathrm{T}$ cells display different suppressive functions in the presence or absence of natural $\mathrm{CD} 4+\mathrm{CD} 25+$ regulatory cells in vivo. the Journal of Immunology 170, 5008-5017 (2003).

182. Fontenot, J.D., et al. Regulatory $\mathrm{T}$ cell lineage specification by the forkhead transcription factor foxp3. Immunity 22, 329-341 (2005).

183. Petersen, T.R., et al. A chimeric T cell receptor with super-signaling properties. Int Immunol 16, 889-894 (2004).

184. Barnden, M.J., Allison, J., Heath, W.R. \& Carbone, F.R. Defective TCR expression in transgenic mice constructed using cDNA-based alpha- and beta- 
chain genes under the control of heterologous regulatory elements. Immunol Cell Biol 76, 34-40 (1998).

185. Kontgen, F., Suss, G., Stewart, C., Steinmetz, M. \& Bluethmann, H. Targeted disruption of the MHC class II Aa gene in C57BL/6 mice. Int Immunol 5, 957964 (1993).

186. Baban, B., et al. Indoleamine 2,3-dioxygenase expression is restricted to fetal trophoblast giant cells during murine gestation and is maternal genome specific. Journal of Reproductive Immunology 61, 67-77 (2004).

187. Dalton, D.K., et al. Multiple defects of immune cell function in mice with disrupted interferon-gamma genes. Science 259, 1739-1742 (1993).

188. Quah, B.J., Warren, H.S. \& Parish, C.R. Monitoring lymphocyte proliferation in vitro and in vivo with the intracellular fluorescent dye carboxyfluorescein diacetate succinimidyl ester. Nat Protoc 2, 2049-2056 (2007).

189. Trahtemberg, U., Atallah, M., Krispin, A., Verbovetski, I. \& Mevorach, D. Calcium, leukocyte cell death and the use of annexin V: fatal encounters. Apoptosis 12, 1769-1780 (2007).

190. Hoffmann, P., et al. Isolation of CD4+CD25+ regulatory $\mathrm{T}$ cells for clinical trials. Biol Blood Marrow Transplant 12, 267-274 (2006).

191. Proft, T. \& Fraser, J.D. Bacterial superantigens. Clin Exp Immunol 133, 299-306 (2003).

192. Li, H., Llera, A., Malchiodi, E.L. \& Mariuzza, R.A. The structural basis of T cell activation by superantigens. Annu Rev Immunol 17, 435-466 (1999).

193. Dickgreber, N., et al. Targeting antigen to MHC class II molecules promotes efficient cross-presentation and enhances immunotherapy. J Immunol 182, 1260-1269 (2009).

194. Gallina, G., et al. Tumors induce a subset of inflammatory monocytes with immunosuppressive activity on CD8+ T cells. J Clin Invest 116, 2777-2790 (2006).

195. Li, P.L., Tiedemann, R.E., Moffat, S.L. \& Fraser, J.D. The superantigen streptococcal pyrogenic exotoxin C (SPE-C) exhibits a novel mode of action. $J$ Exp Med 186, 375-383 (1997).

196. Lagasse, E. \& Weissman, I.L. Flow cytometric identification of murine neutrophils and monocytes. J Immunol Methods 197, 139-150 (1996). 
197. Daley, J.M., Thomay, A.A., Connolly, M.D., Reichner, J.S. \& Albina, J.E. Use of Ly6G-specific monoclonal antibody to deplete neutrophils in mice. $J$ Leukoc Biol 83, 64-70 (2008).

198. Invitrogen. Dynabeads ${ }^{\circledR}$ Mouse CD3/CD28 T Cell Expander. (Invitrogen, 2008).

199. Serafini, P., et al. Phosphodiesterase-5 inhibition augments endogenous antitumor immunity by reducing myeloid-derived suppressor cell function. $J$ Exp Med 203, 2691-2702 (2006).

200. Bronte, V., et al. IL-4-induced arginase 1 suppresses alloreactive $\mathrm{T}$ cells in tumor-bearing mice. J Immunol 170, 270-278 (2003).

201. Kusmartsev, S.A., Li, Y. \& Chen, S.H. Gr-1+ myeloid cells derived from tumorbearing mice inhibit primary $\mathrm{T}$ cell activation induced through $\mathrm{CD} 3 / \mathrm{CD} 28$ costimulation. J Immunol 165, 779-785 (2000).

202. Gajewski, T.F. \& Fitch, F.W. Anti-proliferative effect of IFN-gamma in immune regulation. IV. Murine CTL clones produce IL-3 and GM-CSF, the activity of which is masked by the inhibitory action of secreted IFN-gamma. $J$ Immunol 144, 548-556 (1990).

203. Gajewski, T.F., Joyce, J. \& Fitch, F.W. Antiproliferative effect of IFN-gamma in immune regulation. III. Differential selection of TH1 and TH2 murine helper T lymphocyte clones using recombinant IL-2 and recombinant IFN-gamma. $J$ Immunol 143, 15-22 (1989).

204. Gajewski, T.F., Goldwasser, E. \& Fitch, F.W. Anti-proliferative effect of IFNgamma in immune regulation. II. IFN-gamma inhibits the proliferation of murine bone marrow cells stimulated with IL-3, IL-4, or granulocytemacrophage colony-stimulating factor. J Immunol 141, 2635-2642 (1988).

205. Gajewski, T.F. \& Fitch, F.W. Anti-proliferative effect of IFN-gamma in immune regulation. I. IFN-gamma inhibits the proliferation of Th2 but not Th1 murine helper T lymphocyte clones. J Immunol 140, 4245-4252 (1988).

206. Mellor, A.L. \& Munn, D.H. Ido expression by dendritic cells: tolerance and tryptophan catabolism. Nat Rev Immunol 4, $762-774$ (2004).

207. Taylor, M.W. \& Feng, G.S. Relationship between interferon-gamma, indoleamine 2,3-dioxygenase, and tryptophan catabolism. FASEB $J \mathbf{5}, 2516$ $2522(1991)$. 
208. Munn, D.H., et al. Inhibition of $\mathrm{T}$ cell proliferation by macrophage tryptophan catabolism. J Exp Med 189, 1363-1372 (1999).

209. Hwu, P., et al. Indoleamine 2,3-dioxygenase production by human dendritic cells results in the inhibition of T cell proliferation. J Immunol 164, 3596-3599 (2000).

210. Grohmann, U., Fallarino, F. \& Puccetti, P. Tolerance, DCs and tryptophan: much ado about IDO. Trends in Immunology 24, 242-248 (2003).

211. Radvanyi, L.G., Mills, G.B. \& Miller, R.G. Religation of the T cell receptor after primary activation of mature $\mathrm{T}$ cells inhibits proliferation and induces apoptotic cell death. J Immunol 150, 5704-5715 (1993).

212. Bunt, S.K., et al. Reduced inflammation in the tumor microenvironment delays the accumulation of myeloid-derived suppressor cells and limits tumor progression. Cancer Res 67, 10019-10026 (2007).

213. Stewart, T.J., et al. Interferon Regulatory Factor-8 Modulates the Development of Tumor-Induced CD11b+Gr-1+Myeloid Cells. Journal of Cellular and Molecular Medicine 9999(2009).

214. Rossner, S., et al. Myeloid dendritic cell precursors generated from bone marrow suppress $\mathrm{T}$ cell responses via cell contact and nitric oxide production in vitro. Eur J Immunol 35, 3533-3544 (2005).

215. Moore, S.C., Shaw, M.A. \& Soderberg, L.S. Transforming growth factor-beta is the major mediator of natural suppressor cells derived from normal bone marrow. J Leukoc Biol 52, 596-601 (1992).

216. Brem-Exner, B.G., et al. Macrophages driven to a novel state of activation have anti-inflammatory properties in mice. J Immunol 180, 335-349 (2008).

217. Gordon, S. \& Taylor, P.R. Monocyte and macrophage heterogeneity. Nat Rev Immunol 5, 953-964 (2005).

218. Lin, M.L., et al. Selective suicide of cross-presenting CD8+ dendritic cells by cytochrome c injection shows functional heterogeneity within this subset. Proc Natl Acad Sci U S A 105, 3029-3034 (2008).

219. Maatta, J.A., Sjoholm, U.R., Nygardas, P.T., Salmi, A.A. \& Hinkkanen, A.E. Neutrophils secreting tumor necrosis factor alpha infiltrate the central nervous system of BALB/c mice with experimental autoimmune encephalomyelitis. $J$ Neuroimmunol 90, 162-175 (1998). 
220. Zehntner, S.P., et al. Neutrophils that infiltrate the central nervous system regulate T cell responses. J Immunol 174, 5124-5131 (2005).

221. McColl, S.R., et al. Treatment with anti-granulocyte antibodies inhibits the effector phase of experimental autoimmune encephalomyelitis. J Immunol 161, 6421-6426 (1998).

222. Nygardas, P.T., Maatta, J.A. \& Hinkkanen, A.E. Chemokine expression by central nervous system resident cells and infiltrating neutrophils during experimental autoimmune encephalomyelitis in the BALB/c mouse. Eur $J$ Immunol 30, 1911-1918 (2000).

223. Yanagisawa, K., et al. Hyporesponsiveness to natural killer T-cell ligand alphagalactosylceramide in cancer-bearing state mediated by CD11b+Gr-1+ cells producing nitric oxide. Cancer Res 66, 11441-11446 (2006).

224. Nagaraj, S., et al. Altered recognition of antigen is a mechanism of CD8+ T cell tolerance in cancer. Nat Med 13, 828-835 (2007).

225. Claassen, I., Van Rooijen, N. \& Claassen, E. A new method for removal of mononuclear phagocytes from heterogeneous cell populations in vitro, using the liposome-mediated macrophage 'suicide' technique. J Immunol Methods 134, 153-161 (1990).

226. Nikolic, T., Geutskens, S.B., van Rooijen, N., Drexhage, H.A. \& Leenen, P.J. Dendritic cells and macrophages are essential for the retention of lymphocytes in (peri)-insulitis of the nonobese diabetic mouse: a phagocyte depletion study. Lab Invest 85, 487-501 (2005).

227. Tran, E.H., Hoekstra, K., van Rooijen, N., Dijkstra, C.D. \& Owens, T. Immune invasion of the central nervous system parenchyma and experimental allergic encephalomyelitis, but not leukocyte extravasation from blood, are prevented in macrophage-depleted mice. J Immunol 161, 3767-3775 (1998).

228. van Rooijen, N. \& van Kesteren-Hendrikx, E. Clodronate liposomes: perspectives in research and therapeutics. J Liposome Res 12, 81-94 (2002).

229. Van Rooijen, N. \& Sanders, A. Liposome mediated depletion of macrophages: mechanism of action, preparation of liposomes and applications. J Immunol Methods 174, 83-93 (1994).

230. Rose, N.R. The adjuvant effect in infection and autoimmunity. Clin Rev Allergy Immunol 34, 279-282 (2008). 
231. Bettelli, E., Korn, T., Oukka, M. \& Kuchroo, V.K. Induction and effector functions of TH17 cells. Nature 453, 1051-1057 (2008).

232. Marx, J. Cancer immunology. Cancer's bulwark against immune attack: MDS cells. Science 319, 154-156 (2008).

233. Dow, S.W. \& Potter, T.A. Expression of bacterial superantigen genes in mice induces localized mononuclear cell inflammatory responses. J Clin Invest 99, 2616-2624 (1997).

234. Saloga, J., et al. Cutaneous exposure to the superantigen staphylococcal enterotoxin B elicits a T-cell-dependent inflammatory response. J Invest Dermatol 106, 982-988 (1996).

235. Brodnicki, T.C., Quirk, F. \& Morahan, G. A susceptibility allele from a nondiabetes-prone mouse strain accelerates diabetes in NOD congenic mice. Diabetes 52, 218-222 (2003).

236. Zorzella, S.F., Seger, J., Martins, D.R., Pelizon, A.C. \& Sartori, A. Resistance to experimental autoimmune encephalomyelitis development in Lewis rats from a conventional animal facility. Mem Inst Oswaldo Cruz 102, 931-936 (2007).

237. Dugast, A.S., et al. Myeloid-derived suppressor cells accumulate in kidney allograft tolerance and specifically suppress effector $\mathrm{T}$ cell expansion. $J$ Immunol 180, 7898-7906 (2008).

238. Ochoa, A.C., Zea, A.H., Hernandez, C. \& Rodriguez, P.C. Arginase, prostaglandins, and myeloid-derived suppressor cells in renal cell carcinoma. Clin Cancer Res 13, 721s-726s (2007).

239. Sinha, P., Clements, V.K., Bunt, S.K., Albelda, S.M. \& Ostrand-Rosenberg, S. Cross-Talk between Myeloid-Derived Suppressor Cells and Macrophages Subverts Tumor Immunity toward a Type 2 Response. The Journal of Immunology 179, 977 (2007).

240. Talmadge, J.E. Pathways mediating the expansion and immunosuppressive activity of myeloid-derived suppressor cells and their relevance to cancer therapy. Clin Cancer Res 13, 5243-5248 (2007).

241. Umemura, N., et al. Tumor-infiltrating myeloid-derived suppressor cells are pleiotropic-inflamed monocytes/macrophages that bear M1- and M2-type characteristics. J Leukoc Biol 83, 1136-1144 (2008).

242. Miller, A., Lider, O., al-Sabbagh, A. \& Weiner, H.L. Suppression of experimental autoimmune encephalomyelitis by oral administration of myelin 
basic protein. V. Hierarchy of suppression by myelin basic protein from different species. J Neuroimmunol 39, 243-250 (1992).

243. Clanet, M. \& Montalban, X. The Future of Multiple Sclerosis therapies. Proceedings of the MS Forum Modern Management Workshop Feb, 2006, 1-63 (2006).

244. Smilek, D.E., et al. A single amino acid change in a myelin basic protein peptide confers the capacity to prevent rather than induce experimental autoimmune encephalomyelitis. Proc Natl Acad Sci U S A 88, 9633-9637 (1991).

245. Weiner, H.L. Oral tolerance, an active immunologic process mediated by multiple mechanisms. J Clin Invest 106, 935-937 (2000).

246. Kappos, L., et al. Induction of a non-encephalitogenic type $2 \mathrm{~T}$ helper-cell autoimmune response in multiple sclerosis after administration of an altered peptide ligand in a placebo-controlled, randomized phase II trial. The Altered Peptide Ligand in Relapsing MS Study Group. Nat Med 6, 1176-1182 (2000).

247. Bielekova, B., et al. Encephalitogenic potential of the myelin basic protein peptide (amino acids 83-99) in multiple sclerosis: results of a phase II clinical trial with an altered peptide ligand. Nat Med 6, 1167-1175 (2000).

248. Kusmartsev, S., et al. All-trans-retinoic acid eliminates immature myeloid cells from tumor-bearing mice and improves the effect of vaccination. Cancer Res 63, 4441-4449 (2003).

249. Pak, A.S., Ip, G., Wright, M.A. \& Young, M.R. Treating tumor-bearing mice with low-dose gamma-interferon plus tumor necrosis factor alpha to diminish immune suppressive granulocyte-macrophage progenitor cells increases responsiveness to interleukin 2 immunotherapy. Cancer Res 55, 885-890 (1995).

250. Millington, O.R., Mowat, A.M. \& Garside, P. Induction of bystander suppression by feeding antigen occurs despite normal clonal expansion of the bystander T cell population. J Immunol 173, 6059-6064 (2004).

251. Vanderlugt, C.J. \& Miller, S.D. Epitope spreading. Curr Opin Immunol 8, 831836 (1996).

252. Vanderlugt, C.L. \& Miller, S.D. Epitope spreading in immune-mediated diseases: implications for immunotherapy. Nat Rev Immunol 2, 85-95 (2002). 


\section{Appendix 1: Antibodies Used for Flow Cytometry}

\begin{tabular}{|c|c|c|c|c|}
\hline Antigen & Clone & Source & Isotype & Fluorophore \\
\hline CD4 & GK1.5 & Pharmingen & Rat IgG2b, $\kappa$ & Alexa 647, PE, PerCP \\
\hline CD8 & 2.43 & homemade & Rat IgG2b, $\kappa$ & Alexa $647, \mathrm{PE}$ \\
\hline CD11b & $\mathrm{M} 1 / 70$ & Pharmingen & Rat IgG2b, $\kappa$ & PE, Alexa 647 \\
\hline CD18 & M18/2 & eBioscience & Rat IgG2a, $\kappa$ & Alexa 647 \\
\hline $\mathrm{IA}^{\mathrm{b}}$ & M5/114 & Pharmingen & Rat IgG2b, $\kappa$ & PE, Alexa 647 \\
\hline $\mathrm{IA}^{\mathrm{q}}$ & KH116 & Pharmingen & Mouse IgG2b, $\kappa$ & Biotin \\
\hline CD11c & N418 & homemade & $\begin{array}{l}\text { Armenian } \\
\text { hamster IgG }\end{array}$ & Biotin \\
\hline $\mathrm{F} 4 / 80$ & BM8 & eBioscience & Rat IgG2a, $\kappa$ & Biotin, Alexa 488 \\
\hline Gr-1 & RB6-8C5 & Pharmingen & Rat IgG2b, $\kappa$ & PE, Alexa 647 \\
\hline B220 & RA3-6B2 & homemade & Rat IgG2a, $\kappa$ & Alexa 647, Alexa 488 \\
\hline CD19 & eBio1D3 & eBioscience & Rat IgG2a, $\kappa$ & Biotin, Alexa 647 \\
\hline PDCA-1 & JF05-1C2. & Miltenyi & Rat IgG2b, $\kappa$ & APC \\
\hline CD40 & $3 / 23$ & Pharmingen & Rat IgG2a, $\kappa$ & $\overline{\mathrm{PE}}$ \\
\hline CD25 & $\begin{array}{l}\text { PC61 } \\
\text { 7D4 }\end{array}$ & $\begin{array}{l}\text { Pharmingen } \\
\text { Pharmingen }\end{array}$ & $\begin{array}{l}\text { Rat IgG1, } \lambda \\
\text { Rat IgG1, } \lambda\end{array}$ & $\begin{array}{l}\text { PE, Alexa } 488 \\
\text { PE }\end{array}$ \\
\hline Foxp3 & FJK-16s & eBioscience & Rat IgG2a, $\kappa$ & PE, APC, Alexa 488 \\
\hline Ly6C & AL-21 & $\mathrm{BD}$ & Rat IgM, $\kappa$ & Biotin \\
\hline Ly6G & $1 \mathrm{~A} 8$ & $\mathrm{BD}$ & Rat IgG2a, $\kappa$ & Alexa 647, Biotin \\
\hline CD80 & 16-10A1 & homemade & $\begin{array}{l}\text { Armenian } \\
\text { hamster IgG }\end{array}$ & Biotin \\
\hline CD86 & PO3.1 & eBioscience & Rat IgG2b, $\kappa$ & Alexa 647 \\
\hline CD45.2 & 104 & eBioscience & Mouse IgG2a, $\kappa$ & $\mathrm{PE}$ \\
\hline CD16/CD32 & $2.4 \mathrm{G} 2$ & homemade & Rat IgG2b, $\kappa$ & Alexa 647 \\
\hline VLA-4 & R1-2 & eBioscience & Rat IgG2b, $\kappa$ & FITC \\
\hline Thy1.2 & $53-2.1$ & homemade & Rat IgG2a,,$\kappa$ & Biotin, Alexa 647 \\
\hline CD45 & $30-F 11$ & Pharmingen & Rat IgG2b, $\kappa$ & PerCP \\
\hline$\overline{\text { CD62L }}$ & MEL-14 & eBioscience & Rat IgG2a, $\kappa$ & PE, Alexa 647 \\
\hline
\end{tabular}




\begin{tabular}{|lllll|}
\hline CD69 & H1.2F3 & eBioscience & $\begin{array}{l}\text { Armenian } \\
\text { hamster IgG }\end{array}$ & PE, Alexa 647 \\
\hline CD1d & 1B1 & Pharmingen & Rat IgG2b, $\kappa$ & PE \\
\hline
\end{tabular}




\section{Appendix 2: Validation of DM-MOG ${ }_{35-55}$ Treatment for EAE}

\begin{tabular}{|c|c|c|c|c|c|c|c|c|c|}
\hline $\begin{array}{l}\text { Exp. } \\
\text { No }\end{array}$ & Treatment & Carrier & $\begin{array}{l}\text { Injection } \\
\text { sites }\end{array}$ & $\begin{array}{l}\text { Injection } \\
\text { time (days) }\end{array}$ & Dose & Incidence $\%$ & $\begin{array}{c}\text { Mean day of } \\
\text { onset } \pm \text { SD }\end{array}$ & $\begin{array}{l}\text { Mean max. } \\
\text { severity } \pm \text { SD }\end{array}$ & Mortality \\
\hline \multirow{4}{*}{1} & PBS & - & - & - & - & $(5 / 5) 100 \%$ & $21 \pm 8.75$ & $3.5 \pm 0.35$ & $(0 / 5) 0 \%$ \\
\hline & $\mathrm{DM}^{-M} \mathrm{GG}_{35-55}$ & CFA & Flanks & 0 & $2 \mu \mathrm{g}$ & $(1 / 5) 20 \%$ & 15 & 4 & $(0 / 5) 0 \%$ \\
\hline & DM-MOG $_{35-55}$ & CFA & Neck & 0 & $2 \mu \mathrm{g}$ & $(3 / 5) 60 \%$ & $21 \pm 6.56$ & $3.17 \pm 0.76$ & $(0 / 5) 0 \%$ \\
\hline & DM-MOG $_{35-55}$ & IFA & Neck & 0 & $2 \mu \mathrm{g}$ & $(3 / 5) 60 \%$ & $16.67 \pm 1.15$ & $3.67 \pm 0.58$ & $(0 / 5) 0 \%$ \\
\hline \multirow{4}{*}{2} & PBS & - & - & - & - & $(5 / 5) 100 \%$ & $14 \pm 2.83$ & $4.6 \pm 0.41$ & $(2 / 5) 40 \%$ \\
\hline & DM-MOG $_{35-55}$ & CFA & Flanks & 0 & $1 \mu \mathrm{g}$ & $(4 / 5) 80 \%$ & $19 \pm 7.16$ & $4.625 \pm 0.48$ & $(2 / 5) 40 \%$ \\
\hline & $\mathrm{DM}^{-\mathrm{MOG}_{35-55}}$ & CFA & Flanks & 0 & $2 \mu \mathrm{g}$ & $(3 / 5) 60 \%$ & $14.67 \pm 0.58$ & $3.17 \pm 0.29$ & $(0 / 5) 0 \%$ \\
\hline & $\mathrm{DM}^{-\mathrm{MOG}_{35-55}}$ & CFA & Flanks & 0 & $5 \mu \mathrm{g}$ & $(3 / 5) 60 \%$ & $17.67 \pm 6.35$ & $3.17 \pm 1.04$ & $(0 / 5) 0 \%$ \\
\hline \multirow{3}{*}{3} & DM-OVA $_{323-339}$ & CFA & Flanks & 0 & $1 \mu \mathrm{g}$ & (3/4) $75 \%$ & $13.33 \pm 2.08$ & $4.33 \pm 0.58$ & (1/4) $25 \%$ \\
\hline & PBS & - & Flanks & - & - & $(5 / 5) 100 \%$ & $12.6 \pm 3.13$ & $4.6 \pm 0.55$ & $(3 / 5) 60 \%$ \\
\hline & $\mathrm{DM}^{-\mathrm{MOG}_{35-55}}$ & CFA & Flanks & 0 & $0.5 \mu \mathrm{g}$ & $(5 / 5) 100 \%$ & $14.4 \pm 2.70$ & $4.6 \pm 0.65$ & $(3 / 5) 60 \%$ \\
\hline \multirow{3}{*}{4} & PBS & - & - & - & - & (4/4) $100 \%$ & $14.25 \pm 2.5$ & $4.24 \pm 0.96$ & (2/4) 50\% \\
\hline & DM-MOG $_{35-55}$ & CFA & Flanks & 0 & $2 \mu \mathrm{g}$ & $(2 / 4) 50 \%$ & $25.5 \pm 7.78$ & $2.75 \pm 0.35$ & (0.4) 50\% \\
\hline & $\mathrm{DM}$ & CFA & Flanks & 0 & $2 \mu \mathrm{g}$ & (5/5) 100\% & $13.8 \pm 0.84$ & $5 \pm 0$ & $\begin{array}{c}(5 / 5) \\
100 \%\end{array}$ \\
\hline \multirow{3}{*}{5} & PBS & PBS & Neck & $-7 \sim-1$ & - & $(5 / 5) 100 \%$ & $14.4 \pm 2.07$ & $4.7 \pm 0.27$ & $(3 / 5) 60 \%$ \\
\hline & DM-MOG $_{35-55}$ & PBS & Neck & $-7 \sim-1$ & $1 \mu \mathrm{g} /$ day & $(4 / 5) 80 \%$ & $20.25 \pm 4.78$ & $4 \pm 0$ & $(0 / 5) 0 \%$ \\
\hline & DM & PBS & Neck & $-7 \sim-1$ & $1 \mu \mathrm{g} / \mathrm{day}$ & $(5 / 5) 100 \%$ & $12.6 \pm 0.89$ & $4.8 \pm 0.27$ & $(3 / 5) 60 \%$ \\
\hline \multirow{2}{*}{6} & PBS & - & - & - & - & $(4 / 5) 80 \%$ & $20 \pm 1.41$ & $4.25 \pm 0.65$ & $(1 / 5) 20 \%$ \\
\hline & DM-MOG $_{1-117}$ & IFA & Neck & 5 & $2 \mu \mathrm{g}$ & $(5 / 5) 100 \%$ & $22.2 \pm 4.71$ & $4.1 \pm 0.65$ & $(0 / 5) 0 \%$ \\
\hline \multirow[t]{2}{*}{7} & $\mathrm{IgG}$ & IFA & Neck & 5 & - & $(4 / 5) 80 \%$ & $25 \pm 6.98$ & $4 \pm 0$ & $(0 / 5) 0 \%$ \\
\hline & IgG+DM-MOG $35-55$ & IFA & Neck & 5 & $1 \mu \mathrm{g}$ & $(4 / 5) 80 \%$ & $23.6 \pm 1.95$ & $4.25 \pm 0.65$ & $(1 / 5) 20 \%$ \\
\hline
\end{tabular}




\begin{tabular}{|c|c|c|c|c|c|c|c|c|c|}
\hline $\begin{array}{c}\text { Exp. } \\
\text { No }\end{array}$ & Treatment & Carrier & $\begin{array}{l}\text { Injection } \\
\text { sites }\end{array}$ & $\begin{array}{l}\text { Injection } \\
\text { time (days) }\end{array}$ & Dose & Incidence $\%$ & $\begin{array}{c}\text { Mean day of } \\
\text { onset } \pm \text { SD }\end{array}$ & $\begin{array}{l}\text { Mean max. } \\
\text { severity } \pm \text { SD }\end{array}$ & Mortality \\
\hline & PC61 & IFA & Neck & 5 & - & (3/3) $100 \%$ & $21 \pm 6.28$ & $4.33 \pm 0.58$ & $\begin{array}{c}(1 / 3) \\
33.33 \%\end{array}$ \\
\hline & PC61+DM-MOG $35-55$ & IFA & Neck & 5 & $1 \mu \mathrm{g}$ & $(5 / 5) 100 \%$ & $17.33 \pm 2.89$ & $4.80 \pm 0.27$ & $(3 / 5) 60 \%$ \\
\hline \multirow{4}{*}{8} & PBS & - & Neck & - & - & $(4 / 5) 80 \%$ & $17 \pm 8.12$ & $4.13 \pm 0.25$ & $(0 / 5) 0 \%$ \\
\hline & $\mathrm{DM}^{-\mathrm{MOG}_{35-55}}$ & IFA & Neck & -1 & $100 \mathrm{ng}$ & $(3 / 5) 60 \%$ & $15.67 \pm 3.06$ & $3.33 \pm 1.15$ & $(0 / 5) 0 \%$ \\
\hline & $\mathrm{DM}^{-\mathrm{MOG}_{35-55}}$ & IFA & Neck & 1 & 100ng & $(5 / 5) 100 \%$ & $18.25 \pm 9.46$ & $4.38 \pm 0.48$ & $(1 / 5) 20 \%$ \\
\hline & $\mathrm{DM}^{-\mathrm{MOG}_{35-55}}$ & IFA & Neck & 5 & 100 ng & $(3 / 5) 60 \%$ & $16 \pm 6.73$ & $4 \pm 0$ & $(0 / 5) 0 \%$ \\
\hline \multirow{4}{*}{9} & PBS & - & - & - & - & $(5 / 5) 100 \%$ & $24 \pm 10.15$ & $4 \pm 0.71$ & $(1 / 5) 20 \%$ \\
\hline & DM-MOG $_{35-55}$ & IFA & Neck & 5 & $1 \mu \mathrm{g}$ & $(5 / 5) 100 \%$ & $15.2 \pm 2.49$ & $3.7 \pm 0.91$ & $(0 / 5) 0 \%$ \\
\hline & PC61 & IFA & - & - & - & $(5 / 5) 100 \%$ & $16.6 \pm 2.41$ & $4.6 \pm 0.55$ & $(3 / 5) 60 \%$ \\
\hline & PC61+ DM-MOG $35-55$ & IFA & Neck & 5 & $1 \mu \mathrm{g}$ & $(4 / 5) 80 \%$ & $17.25 \pm 2.06$ & $4.5 \pm 1.00$ & $(3 / 5) 60 \%$ \\
\hline \multirow{4}{*}{10} & PBS & - & - & - & - & $(5 / 5) 100 \%$ & $13.8 \pm 1.64$ & $4.2 \pm 0.45$ & $(1 / 5) 20 \%$ \\
\hline & DM-MOG $_{35-55}$ & IFA & Neck & 5 & 200ng & $(5 / 5) 100 \%$ & $14.2 \pm 0.84$ & $4.1 \pm 1.02$ & $(2 / 5) 40 \%$ \\
\hline & $\mathrm{DM}_{-\mathrm{MOG}_{35-55}}$ & IFA & Neck & 5 & $1 \mu \mathrm{g}$ & $(5 / 5) 100 \%$ & $12.2 \pm 0.45$ & $4.2 \pm 0.45$ & $(1 / 5) 20 \%$ \\
\hline & $\mathrm{DM}^{-\mathrm{MOG}_{35-55}}$ & IFA & Neck & 5 & $5 \mu \mathrm{g}$ & $(4 / 5) 80 \%$ & $13.75 \pm 2.21$ & $4.25 \pm 0.50$ & $(1 / 5) 20 \%$ \\
\hline \multirow{3}{*}{11} & PBS & - & - & - & - & $(5 / 5) 100 \%$ & $16.4 \pm 5.03$ & $4 \pm 1.00$ & $(2 / 5) 40 \%$ \\
\hline & $\mathrm{DM}^{-\mathrm{MOG}_{35-55}}$ & IFA & Neck & 5 & 100ng & $(5 / 5) 100 \%$ & $24.8 \pm 6.57$ & $3.3 \pm 1.30$ & $(0 / 5) 0 \%$ \\
\hline & $\mathrm{DM}^{-\mathrm{MOG}_{35-55}}$ & IFA & Neck & 5 & $1 \mu \mathrm{g}$ & $(5 / 5) 100 \%$ & $17.6 \pm 2.30$ & $3.5 \pm 0.50$ & $(0 / 5) 0 \%$ \\
\hline \multirow{3}{*}{12} & PBS & - & - & - & - & $(5 / 5) 100 \%$ & $13.8 \pm 2.77$ & $4.1 \pm 1.02$ & $(2 / 5) 40 \%$ \\
\hline & PC61+ DM-MOG $35-55$ & IFA & Neck & 5 & $1 \mu \mathrm{g}$ & $(5 / 5) 100 \%$ & $13 \pm 2$ & $4.8 \pm 0.45$ & $(4 / 5) 80 \%$ \\
\hline & IgG+DM-MOG $35-55$ & IFA & Neck & 5 & $1 \mu \mathrm{g}$ & $(5 / 5) 100 \%$ & $14.4 \pm 0.89$ & $4.2 \pm 0.45$ & $(1 / 5) 20 \%$ \\
\hline \multirow{2}{*}{13} & PBS & - & - & - & - & $(5 / 5) 100 \%$ & $12.6 \pm 0.55$ & $4.6 \pm 0.55$ & $(3 / 5) 60 \%$ \\
\hline & $\mathrm{DM}^{-\mathrm{MOG}_{35-55}}$ & IFA & Neck & 5 & $1 \mu \mathrm{g}$ & $(3 / 5) 60 \%$ & $16.33 \pm 4.04$ & $2.67 \pm 0.58$ & $(0 / 5) 0 \%$ \\
\hline \multirow[t]{4}{*}{14} & PBS & - & - & - & - & $(5 / 5) 100 \%$ & $18.4 \pm 8.44$ & $3.6+0.96$ & $(0 / 5) 0 \%$ \\
\hline & $\mathrm{DM}^{-M^{\prime}}$ & IFA & Neck & 5 & $1 \mu \mathrm{g}$ & $(5 / 5) 100 \%$ & $17.2 \pm 4.76$ & $2.8 \pm 1.60$ & $(0 / 5) 0 \%$ \\
\hline & IgG+DM-MOG $35-55$ & IFA & Neck & 5 & $1 \mu \mathrm{g}$ & $(5 / 5) 100 \%$ & $17.8 \pm 2.68$ & $3.2 \pm 0.76$ & $(0 / 5) 0 \%$ \\
\hline & 2.43 & - & - & - & - & $(4 / 5) 80 \%$ & $17 \pm 0$ & $2.38 \pm 1.60$ & $(0 / 5) 0 \%$ \\
\hline
\end{tabular}




\begin{tabular}{|c|l|c|c|c|c|c|c|c|c|}
\hline $\begin{array}{c}\text { Exp. } \\
\text { No }\end{array}$ & \multicolumn{1}{|c|}{ Treatment } & Carrier & $\begin{array}{c}\text { Injection } \\
\text { sites }\end{array}$ & $\begin{array}{c}\text { Injection } \\
\text { time (days) }\end{array}$ & Dose & Incidence\% & $\begin{array}{c}\text { Mean day of } \\
\text { onset } \pm \text { SD }\end{array}$ & $\begin{array}{c}\text { Mean max. } \\
\text { severity } \pm \text { SD }\end{array}$ & \begin{tabular}{c} 
Mortality \\
\hline
\end{tabular} \\
\hline \multirow{2}{*}{15} & $2.43+\mathrm{DM}_{-} \mathrm{MOG}_{35-55}$ & IFA & Neck & 5 & $1 \mu \mathrm{g}$ & $(5 / 5) 100 \%$ & $20.4 \pm 6.66$ & $2.8 \pm 1.20$ & $(0 / 5) 0 \%$ \\
\cline { 2 - 10 } & $\mathrm{DM}-\mathrm{MOG}_{35-55}$ & - & - & - & - & $(5 / 5) 100 \%$ & $16 \pm 3$ & $3.9 \pm 0.42$ & $(0 / 5) 0 \%$ \\
\hline
\end{tabular}

\section{Intravenous treatment}

\begin{tabular}{|c|l|c|c|c|c|c|c|}
\hline $\begin{array}{c}\text { Exp. } \\
\text { No }\end{array}$ & \multicolumn{1}{|c|}{ Treatment } & $\begin{array}{c}\text { Injection } \\
\text { time (days) }\end{array}$ & Dose & Incidence\% & $\begin{array}{c}\text { Mean day of onset } \\
\pm \text { SD }\end{array}$ & $\begin{array}{c}\text { Mean Max. } \\
\text { severity } \pm \text { SD }\end{array}$ & Mortality\% \\
\hline \multirow{3}{*}{1} & PBS & - & - & $(5 / 5) 100 \%$ & $16.4 \pm 5.03$ & $4 \pm 1.00$ & $(2 / 5) 40 \%$ \\
\cline { 2 - 8 } & DM-MOG $35-55$ & -1 & $100 \mathrm{ng}$ & $(5 / 5) 100 \%$ & $14 \pm 2.74$ & $4.1 \pm 0.55$ & $(1 / 5) 20 \%$ \\
\cline { 2 - 8 } & DM-MOG $_{35-55}$ & -1 & $5 \mu \mathrm{g}$ & $(5 / 5) 100 \%$ & $17 \pm 5.74$ & $3.6 \pm 0.89$ & $(1 / 5) 20 \%$ \\
\hline
\end{tabular}

\section{Intraperitoneal treatment}

\begin{tabular}{|c|c|c|c|c|c|c|c|}
\hline $\begin{array}{c}\text { Exp. } \\
\text { No }\end{array}$ & \multicolumn{1}{|c|}{ Treatment } & $\begin{array}{c}\text { Injection } \\
\text { time (days) }\end{array}$ & Dose & Incidence\% & $\begin{array}{c}\text { Mean day of onset } \\
\pm \text { SD }\end{array}$ & $\begin{array}{c}\text { Mean Max. } \\
\text { severity } \pm \text { SD }\end{array}$ & Mortality\% \\
\hline \multirow{2}{*}{1} & PBS & - & - & $(5 / 5) 100 \%$ & $22.2 \pm 11.23$ & $2.6 \pm 0.96$ & $(0 / 5) 0 \%$ \\
\cline { 2 - 8 } & DM-MOG $35-55$ & 4 & $100 \mathrm{ng}$ & $(5 / 5) 100 \%$ & $15.2 \pm 1.64$ & $4 \pm 0.50$ & $(0 / 5) 0 \%$ \\
\hline
\end{tabular}


Cell Adoptive transfer post DM-MOG $35-55$ immunisation

\begin{tabular}{|c|c|c|c|c|c|c|c|c|c|}
\hline $\begin{array}{l}\text { Exp. } \\
\text { No }\end{array}$ & Cell type & $\begin{array}{c}\text { Doses on } \\
\text { the host }\end{array}$ & $\begin{array}{c}\text { Cell } \\
\text { purification } \\
\text { from host } \\
\text { (days) }\end{array}$ & $\begin{array}{c}\text { Adoptive } \\
\text { transfer post } \\
\text { EAE induction } \\
\text { (days) }\end{array}$ & $\begin{array}{l}\text { Cell } \\
\text { NO. }\end{array}$ & Incidence $\%$ & $\begin{array}{c}\text { Mean day of } \\
\text { onset } \pm \text { SD }\end{array}$ & $\begin{array}{l}\text { Mean max. } \\
\text { severity } \pm \text { SD }\end{array}$ & Mortality\% \\
\hline \multirow{3}{*}{1} & PBS & - & 5 & 5 & - & $(4 / 5) 80 \%$ & $26 \pm 11.75$ & $3.75 \pm 1.5$ & $(2 / 5) 40 \%$ \\
\hline & $\begin{array}{l}\text { DM-MOG } 35-55 \\
\text { CD11b+ cells }\end{array}$ & $2 \mu \mathrm{g}$ & 5 & 5 & $2 \times 10^{5}$ & $(1 / 4) 25 \%$ & 12 & 4 & $(0 / 4) 0 \%$ \\
\hline & Naïve CD11b+ & - & 5 & 5 & $2 \times 10^{5}$ & $(3 / 5) 60 \%$ & $16.33 \pm 2.31$ & $4.16 \pm 0.76$ & $(1 / 5) 20 \%$ \\
\hline \multirow{3}{*}{2} & PBS & - & - & -1 & - & $(3 / 3) 100 \%$ & $12 \pm 1.73$ & $4.5 \pm 0.5$ & (1/3) 33.3\% \\
\hline & $\begin{array}{l}\text { DM-MOG } \\
\text { DLN-55 cells }\end{array}$ & $2 \mu \mathrm{g}$ & 5 & -1 & $1 \times 10^{7}$ & $(3 / 3) 100 \%$ & $13.33 \pm 2.52$ & $4.33 \pm 0.29$ & $(0 / 3) 0 \%$ \\
\hline & $\begin{array}{l}\text { DM-OVA }_{323-339} \\
\text { DLN cells }\end{array}$ & $2 \mu \mathrm{g}$ & 5 & -1 & $1 \times 10^{7}$ & (3/3) 100\% & $14.33 \pm 1.15$ & $4.5 \pm 0$ & $(0 / 3) 0 \%$ \\
\hline \multirow{4}{*}{3} & PBS & - & - & - & - & $(2 / 3) 66 \%$ & $15.50 \pm 0.71$ & $3.75 \pm 1.06$ & $(0 / 3) 100 \%$ \\
\hline & $\begin{array}{l}\text { DM-MOG } 35-55 \\
\text { CD25+ cells }\end{array}$ & $1 \mu \mathrm{g}$ & 5 & -2 & $2.5 \times 10^{5}$ & $(3 / 3) 100 \%$ & $20 \pm 7.81$ & $3.83 \pm 1.26$ & (1/3) 33.3\% \\
\hline & $\begin{array}{l}\text { DM-MOG } 35-55 \\
\text { CD11b+ cells }\end{array}$ & $1 \mu \mathrm{g}$ & 5 & -2 & $2.5 \times 10^{5}$ & $(3 / 3) 100 \%$ & $16.67 \pm 4.04$ & $5 \pm 0$ & (3/3) 100\% \\
\hline & $\begin{array}{l}\text { DM-MOG } 35-55 \\
\text { CD25+ cells } \\
\text { CD11b+ cells }\end{array}$ & $1 \mu \mathrm{g}$ & 5 & -2 & $\begin{array}{c}2.5 \times 10^{5} \\
\text { each }\end{array}$ & $(3 / 3) 100 \%$ & $16 \pm 3$ & $3.5 \pm 0.5$ & $(0 / 3) 100 \%$ \\
\hline \multirow{4}{*}{4} & PBS & - & - & - & - & $(1 / 2) 50 \%$ & 13 & 5 & $(1 / 2) 50 \%$ \\
\hline & $\begin{array}{l}\text { DM-MOG } \\
35-55 \\
\text { F4/80+ cells }\end{array}$ & $1 \mu \mathrm{g}$ & 5 & -2 & $2.5 \times 10^{4}$ & (2/2) $100 \%$ & $13.5 \pm 0.71$ & $5 \pm 0.00$ & $(2 / 2) 100 \%$ \\
\hline & $\begin{array}{l}\text { DM-MOG }_{35-55} \\
\text { CD25+ cells }\end{array}$ & $1 \mu \mathrm{g}$ & 5 & -2 & $2.5 \times 10^{4}$ & $(2 / 2) 100 \%$ & $15.5 \pm 3.54$ & $4 \pm 1.41$ & $(1 / 2) 50 \%$ \\
\hline & $\begin{array}{l}\text { DM-MOG } 35-55 \\
\text { CD25+ cells } \\
\text { F4/80+ cells }\end{array}$ & $1 \mu \mathrm{g}$ & 5 & -2 & $\begin{array}{c}2.5 \times 10^{4} \\
\text { each }\end{array}$ & $(2 / 2) 100 \%$ & $11.5 \pm 0.71$ & $4.25 \pm 1.06$ & $(1 / 2) 50 \%$ \\
\hline
\end{tabular}




\section{Appendix 3: Buffers and Reagents}

\section{Common buffers}

\subsection{PBS (PH 7.2)}

\begin{tabular}{|l|l|l|}
\hline Item & Volume & Supplier \\
\hline PBS $10 \mathrm{X}$ powder & 1 pack & Invitrogen, USA \\
\hline $\mathrm{DH}_{2} \mathrm{O}$ & $1 \mathrm{~L}$ & \\
\hline
\end{tabular}

Adjust $\mathrm{PH}$ to 7.2 by using $\mathrm{NaOH}$ or $\mathrm{HCl}$. The PBS was filter sterilised (Steritop ${ }^{\mathrm{TM}}$,

Millipore, Billerica, Ma, USA) to allow long-term storage. Store at $4^{\circ} \mathrm{C}$.

\subsection{5 M EDTA (PH 7.4)}

\begin{tabular}{|l|l|l|}
\hline Item & Volume & Supplier \\
\hline EDTA & $14.61 \mathrm{~g}(\mathrm{MW}=292.24)$ & Invitrogen, USA \\
\hline $\mathrm{DH}_{2} \mathrm{O}$ & $100 \mathrm{ml}$ & \\
\hline
\end{tabular}

Adjust $\mathrm{PH}$ to 7.4 by using $\mathrm{NaOH}$ or $\mathrm{HCl}$. The buffer was filter sterilised (Steritop ${ }^{\mathrm{TM}}$, Millipore) to allow long-term storage. Store at room temperature.

\subsection{5 M Tris-HCl (PH 6.8)}

\begin{tabular}{|l|l|l|}
\hline Item & Volume & Supplier \\
\hline Tris base & $60.57 \mathrm{~g}(\mathrm{MW}=121.14)$ & Invitrogen, USA \\
\hline $\mathrm{DH}_{2} \mathrm{O}$ & $1 \mathrm{~L}$ & \\
\hline
\end{tabular}

Adjust PH to 6.8 by HCl. The buffer was filter sterilised (Steritop ${ }^{\mathrm{TM}}$, Millipore) to allow long-term storage. Store at room temperature.

\subsection{Tris-HCl (PH 7.4)}

\begin{tabular}{|l|l|l|}
\hline Item & Volume & Supplier \\
\hline Tris base & $\begin{array}{l}242.28 \mathrm{~g} \\
(\mathrm{MW}=121.14)\end{array}$ & Invitrogen, USA \\
\hline $\mathrm{DH}_{2} \mathrm{O}$ & $1 \mathrm{~L}$ & \\
\hline
\end{tabular}

Adjust PH to 7.4 by using HCl. The buffer was filter sterilised (Steritop ${ }^{\mathrm{TM}}$, Millipore) to allow long-term storage. Store at room temperature.

\section{Buffers for tissue processing}

\subsection{Alsevers solution for blood processing}

\begin{tabular}{|l|l|l|}
\hline Item & Volume & Supplier \\
\hline D-glucose & $20.5 \mathrm{~g}(\mathrm{MW}=180.16)$ & AppliChem, Germany \\
\hline Sodium citrate $2 \mathrm{H}_{2} \mathrm{O}$ & $7.9 \mathrm{~g}$ & Sigma-Aldrich, USA \\
\hline $\mathrm{NaCl}$ & $4.2 \mathrm{~g}$ & BDH Laboratory supplies, UK \\
\hline $\mathrm{DH}_{2} \mathrm{O}$ & $1 \mathrm{~L}$ & \\
\hline
\end{tabular}

Adjust $\mathrm{PH}$ to 6.1 by using $1 \mathrm{M}$ citric acid. Store at $4{ }^{\circ} \mathrm{C}$. 


\subsection{Red blood cell lysis buffer}

\begin{tabular}{|l|l|l|}
\hline Item & Volume & Supplier \\
\hline $\mathrm{NH}_{4} \mathrm{Cl}$ & $3.74 \mathrm{~g}(\mathrm{MW}=53.49)$ & BDH Laboratory supplies, UK \\
\hline $2 \mathrm{M} \mathrm{Tris} \mathrm{HCl}(\mathrm{PH} \mathrm{7.4})$ & $4.25 \mathrm{ml}$ & Invitrogen, USA \\
\hline $\mathrm{DH}_{2} \mathrm{O}$ & $500 \mathrm{ml}$ & \\
\hline
\end{tabular}

The buffer was filter sterilised (Steritop ${ }^{\mathrm{TM}}$, Millipore) and stored at room temperature.

\subsection{8\% NaCl buffer for red blood cell lyse}

\begin{tabular}{|l|l|l|}
\hline Item & Volume & Supplier \\
\hline $\mathrm{NaCl}$ & $90 \mathrm{~g}(\mathrm{MW}=58.44)$ & BDH Laboratory supplies, UK \\
\hline $\mathrm{DH}_{2} \mathrm{O}$ & $500 \mathrm{ml}$ & \\
\hline
\end{tabular}

The diluent was filter sterilised (Steritop ${ }^{\mathrm{TM}}$, Millipore) and stored at room temperature.

\subsection{Complete IMDM}

\begin{tabular}{|l|l|l|}
\hline Item & Volume & Supplier \\
\hline FCS & $50 \mathrm{ml}$ & Invitrogen, USA \\
\hline Glutamine & $2 \mathrm{mM}$ & Invitrogen, USA \\
\hline Penicillin G & $100 \mathrm{U} / \mathrm{ml}$ & Invitrogen, USA \\
\hline Streptomycin sulphate & $100 \mu \mathrm{g} / \mathrm{ml}$ & Invitrogen, USA \\
\hline 2-mercaptoethanol & $5 \times 10^{-5} \mathrm{M}$ & Invitrogen, USA \\
\hline IMDM & $1 \mathrm{~L}$ & Invitrogen, USA \\
\hline
\end{tabular}

Store at $4^{\circ} \mathrm{C}$.

\subsection{Heparin solution for peritoneal wash}

\begin{tabular}{|l|l|l|}
\hline Item & Volume & Supplier \\
\hline $\begin{array}{l}\text { Heparin sodium (porcine } \\
\text { mucous) }\end{array}$ & $\begin{array}{l}50 \mu \mathrm{l}(5000 \mathrm{IU} \text { in } 0.2 \\
\mathrm{ml} \text { ampoule) }\end{array}$ & $\begin{array}{l}\text { Hospira Australia Pty Ltd, } \\
\text { Australia }\end{array}$ \\
\hline PBS & $50 \mathrm{ml}$ & As described in 1.1 \\
\hline $0.5 \mathrm{M}$ EDTA (PH 7.4) & $300 \mu \mathrm{l}$ & As described in 1.2 \\
\hline
\end{tabular}

Mix well and store at $4^{\circ} \mathrm{C}$.

\subsection{Hanks solution for cell processing}

\begin{tabular}{|l|l|l|}
\hline Item & Volume & Supplier \\
\hline $\begin{array}{l}\text { Hanks' balanced salt } \\
\text { solution }\end{array}$ & $50 \mathrm{ml}$ & Invitrogen, USA \\
\hline 0.5 M EDTA (PH 7.4) & $200 \mu \mathrm{l}$ & As described in 1.2 \\
\hline
\end{tabular}

Store at room temperature 


\subsection{Cell freezing medium}

\begin{tabular}{|l|l|l|}
\hline Item & Volume & Supplier \\
\hline FCS & $90 \mathrm{ml}$ & Invitrogen, USA \\
\hline Sterile DMSO & $10 \mathrm{ml}$ & Sigma-Aldrich, USA \\
\hline
\end{tabular}

Mix well and store at $-20^{\circ} \mathrm{C}$.

\subsection{Percoll diluent for cell isolation from spinal cord}

\begin{tabular}{|l|l|l|}
\hline Item & Volume & Supplier \\
\hline $10 \mathrm{X}$ sterile PBS & $45 \mathrm{ml}$ & Invitrogen, USA \\
\hline $3 \mathrm{ml} 0.6 \mathrm{M} \mathrm{HCl}$ & $3 \mathrm{ml}$ & Invitrogen, USA \\
\hline $\mathrm{DH}_{2} \mathrm{O}$ & $132 \mathrm{ml}$ & \\
\hline
\end{tabular}

The diluent was filter sterilised (Steritop ${ }^{\mathrm{TM}}$, Millipore) and stored at $4^{\circ} \mathrm{C}$.

\section{Buffers for FACS staining}

\subsection{FACS buffer}

\begin{tabular}{|l|l|l|}
\hline Item & Volume & Supplier \\
\hline Sodium azide & $0.1 \%$ & Invitrogen, USA \\
\hline FCS & $2 \%$ & Invitrogen, USA \\
\hline EDTA & $2 \mathrm{mM}$ & As described in 1.2 \\
\hline PBS & $1 \mathrm{~L}$ & As described in 1.1 \\
\hline
\end{tabular}

Store at room temperature.

\section{2 $\mathrm{ZnCl}_{2}$ buffer for superantigen staining}

\begin{tabular}{|l|l|l|}
\hline Item & Volume & Supplier \\
\hline PBS & $1 \mathrm{~L}$ & As described in 1.1 \\
\hline FCS & $1 \%$ & Invitrogen, USA \\
\hline $\mathrm{ZnCl}_{2}$ & $\begin{array}{l}0.2 \mathrm{mM}, \\
(\mathrm{MW}=136.28)\end{array}$ & Sigma-Aldrich, USA \\
\hline Sodium azide & $0.1 \%$ & Invitrogen, USA \\
\hline
\end{tabular}

The buffer was filter sterilised (Steritop ${ }^{\mathrm{TM}}$, Millipore) and stored at room temperature.

\subsection{X stock solution for DM staining}

\begin{tabular}{|l|l|l|}
\hline Item & Volume & Supplier \\
\hline $\mathrm{MgSO}_{4} \cdot 6 \mathrm{H}_{2} \mathrm{O}$ & $\begin{array}{l}100 \mathrm{mM}, \\
(\mathrm{MW}=223.46)\end{array}$ & $\begin{array}{l}\text { Merk, Whitehouse Station, NJ. } \\
\text { USA }\end{array}$ \\
\hline $\mathrm{MnCl}_{2} \cdot 4 \mathrm{H}_{2} \mathrm{O}$ & $10 \mathrm{mM},(\mathrm{MW}=197.9)$ & Malinchrodt Chemicals, USA \\
\hline $\mathrm{DH}_{2} \mathrm{O}$ & $50 \mathrm{ml}$ & \\
\hline
\end{tabular}

The buffer was filter sterilised (Steritop ${ }^{\mathrm{TM}}$, Millipore) and stored at room temperature. The stock solution was diluted 100X in cIMDM to make working solution. 
3.4 Fixation/Permeabilization buffer for Foxp3 intracellular staining

\begin{tabular}{|l|l|l|}
\hline Item & Volume & Supplier \\
\hline $\begin{array}{l}\text { Fixation/ } \\
\begin{array}{l}\text { Permeabilization } \\
\text { concentrate }\end{array}\end{array}$ & $3 \mathrm{ml}$ & eBioscience, US \\
\hline $\begin{array}{l}\text { Fixation/ } \\
\text { Permeabilization diluent }\end{array}$ & $9 \mathrm{ml}$ & eBioscience, US \\
\hline
\end{tabular}

This buffer was made fresh and was not stored for more than one day.

\subsection{Permeabilization buffer for Foxp3 intracellular staining}

\begin{tabular}{|l|l|l|}
\hline Item & Volume & Supplier \\
\hline $\begin{array}{l}\text { Permeabilization buffer } \\
(10 \mathrm{X})\end{array}$ & $1 \mathrm{ml}$ & eBioscience, US \\
\hline $\mathrm{DH}_{2} \mathrm{O}$ & $9 \mathrm{ml}$ & \\
\hline
\end{tabular}

This buffer was made fresh and was not stored for more than one day.

\subsection{Annexin V binding buffer}

\begin{tabular}{|l|l|l|}
\hline Item & Volume & Supplier \\
\hline HEPES & $10 \mathrm{mM}$ & Invitrogen, USA \\
\hline $\mathrm{NaCl}$ & $140 \mathrm{mM}$ & BDH Laboratory supplies, UK \\
\hline $\mathrm{DH}_{2} \mathrm{O}$ & $200 \mathrm{ml}$ & \\
\hline
\end{tabular}

Adjust $\mathrm{pH}$ to 7.4 and store at $4^{\circ} \mathrm{C}$. This buffer was not stored for more than one month.

\section{Buffers for superantigen coupling}

\subsection{Coupling buffer}

\begin{tabular}{|l|l|l|}
\hline Item & Volume & Supplier \\
\hline Tris- $\mathrm{HCl}(\mathrm{PH} 8.0)$ & $0.2 \mathrm{M}$ & Invitrogen, USA \\
\hline $\mathrm{CuSO}{ }_{4} \cdot 5 \mathrm{H}_{2} \mathrm{O}$ & $20 \mu \mathrm{M}$ & AJAX Chemicals, Australia \\
\hline $\mathrm{DH}_{2} \mathrm{O}$ & $100 \mathrm{ml}$ & \\
\hline
\end{tabular}

Adjust $\mathrm{PH}$ to 8.0 by using $\mathrm{NaOH}$ or $\mathrm{HCl}$. The buffer was filter sterilised with $0.22 \mu \mathrm{m}$ filter (Sartorius, Global Science and Technology Ltd, Germany) and stored at room temperature. 


\section{2 $20 \mathrm{mM}$ sodium phosphate buffer for superantigen storage}

\begin{tabular}{|l|l|l|}
\hline Item & Volume & Supplier \\
\hline $\mathrm{Na}_{2} \mathrm{HPO}_{4}$ & $10 \mathrm{mM}$ & BDH Laboratory supplies, UK \\
\hline $\mathrm{NaH}_{2} \mathrm{PO}_{4}$ & $10 \mathrm{mM}$ & BDH Laboratory supplies, UK \\
\hline $\mathrm{DH}_{2} \mathrm{O}$ & $100 \mathrm{ml}$ & \\
\hline
\end{tabular}

Adjust $\mathrm{PH}$ to 6.0 by using $\mathrm{NaOH}$ or $\mathrm{HCl}$. The buffer was filter sterilised with $0.22 \mu \mathrm{m}$ filter (Sartorius, Global Science and Technology Ltd, Germany) and stored at room temperature.

\section{3 $0.1 \%$ Acetic acid for peptide storage}

\begin{tabular}{|l|l|l|}
\hline Item & Volume & Supplier \\
\hline Acetic acid (99.5\% pure) & $0.1 \mathrm{ml}$ & AJAX Chemicals, Australia \\
\hline $\mathrm{DH}_{2} \mathrm{O}$ & $100 \mathrm{ml}$ & \\
\hline
\end{tabular}

Store at room temperature.

\section{Buffers for NuPAGE ${ }^{\circledR}$ gel}

\subsection{Coomassie Blue}

\begin{tabular}{|l|l|l|}
\hline Item & Volume & Supplier \\
\hline $0.1 \%$ Coomassie blue & $0.5 \mathrm{~g}$ & Bio-Rad, USA \\
\hline $10 \%$ acetic acid & $50 \mathrm{ml}$ & AJAX Chemicals, Australia \\
\hline $45 \%$ methanol & $225 \mathrm{ml}$ & BDH Laboratory supplies, UK \\
\hline $\mathrm{DH}_{2} \mathrm{O}$ & $225 \mathrm{ml}$ & \\
\hline
\end{tabular}

Store at room temperature.

\subsection{Destain solution}

\begin{tabular}{|l|l|l|}
\hline Item & Volume & Supplier \\
\hline $10 \%$ acetic acid & $75 \mathrm{ml}$ & AJAX Chemicals, Australia \\
\hline $45 \%$ methanol & $50 \mathrm{ml}$ & BDH Laboratory supplies, UK \\
\hline $\mathrm{DH}_{2} \mathrm{O}$ & $875 \mathrm{ml}$ & \\
\hline
\end{tabular}

Store at room temperature.

\section{Buffers and solutions for injection}

\subsection{PTxn buffer}

\begin{tabular}{|l|l|l|}
\hline Item & Volume & Supplier \\
\hline $\mathrm{NaCl}$ & $0.5 \mathrm{M}$ & BDH Laboratory supplies, UK \\
\hline Tris $\mathrm{HCl}(\mathrm{PH} 7.4)$ & $15 \mathrm{mM}$ & Invitrogen, USA \\
\hline Triton-X-100 & $0.017 \%$ & Sigma-Aldrich, USA \\
\hline $\mathrm{DH}_{2} \mathrm{O}$ & $1 \mathrm{~L}$ & \\
\hline
\end{tabular}

The buffer was filter sterilised (Steritop ${ }^{\mathrm{TM}}$, Millipore) and stored at room temperature. 


\subsection{Ketamine/Xylazine anaethetic}

\begin{tabular}{|l|l|l|}
\hline Item & Volume & Supplier \\
\hline Ketamine $(100 \mathrm{mg} / \mathrm{ml})$ & $10 \mathrm{ml}$ & $\begin{array}{l}\text { Phoenix Pharm } \\
\text { Distributors Ltd, New Zealand }\end{array}$ \\
\hline Xylazine $(20 \mathrm{mg} / \mathrm{ml})$ & $1.5 \mathrm{ml}$ & $\begin{array}{l}\text { Phoenix Pharm } \\
\text { Distributors Ltd, New Zealand }\end{array}$ \\
\hline PBS & $103 \mathrm{ml}$ & As described in 1.1 \\
\hline
\end{tabular}

The ketamine/xylazine were mixed to make a 10X stock of anaesthetic. The stock solution was stored at room temperature and diluted 10 fold prior to use.

\subsection{Pentobarbitone anaethetic}

\begin{tabular}{|l|l|l|}
\hline Item & Volume & Supplier \\
\hline Pentobarbitone & $80 \mu \mathrm{l}$ & $\begin{array}{l}\text { National Veterinary Supplies } \\
\text { Ltd, New Zealand }\end{array}$ \\
\hline PBS & $5 \mathrm{ml}$ & As described in 1.1 \\
\hline
\end{tabular}

The stock of pentobarbitone was stored at room temperature and diluted prior to use.

\section{Buffers for cell separation}

\subsection{Automacs running buffer}

\begin{tabular}{|l|l|l|}
\hline Item & Volume & Supplier \\
\hline FCS & $1 \%$ & Invitrogen, USA \\
\hline EDTA & $2 \mathrm{mM}$ & As described in 1.2 \\
\hline PBS & $1 \mathrm{~L}$ & As described in 1.1 \\
\hline
\end{tabular}

The buffer was filter sterilised (Steritop ${ }^{\mathrm{TM}}$, Millipore) and stored at $4^{\circ} \mathrm{C}$.

\subsection{Automacs rinse buffer}

\begin{tabular}{|l|l|l|}
\hline Item & Volume & Supplier \\
\hline EDTA & $2 \mathrm{mM}$ & As described in 1.2 \\
\hline PBS & $1 \mathrm{~L}$ & As described in 1.1 \\
\hline
\end{tabular}

The buffer was filter sterilised (Steritop ${ }^{\mathrm{TM}}$, Millipore) and stored at $4^{\circ} \mathrm{C}$.

\subsection{Dynal beads separation buffer}

\begin{tabular}{|l|l|l|}
\hline Item & Volume & Supplier \\
\hline FCS & $1 \%(1 \mathrm{ml})$ & Invitrogen, USA \\
\hline Sodium Citrate & $0.6 \%(0.6 \mathrm{~g})$ & Sigma-Aldrich, USA \\
\hline HBSS & $100 \mathrm{ml}$ & Invitrogen, USA \\
\hline
\end{tabular}

The buffer was filter sterilised (Steritop ${ }^{\mathrm{TM}}$, Millipore) and stored at $4^{\circ} \mathrm{C}$. 


\subsection{Dynalbeads ${ }^{\circledR}$ FlowComp $^{\mathrm{TM}}$ isolation buffer}

\begin{tabular}{|l|l|l|}
\hline Item & Volume & Supplier \\
\hline BSA & $0.1 \%(0.5 \mathrm{~g})$ & \\
\hline EDTA & $2 \mathrm{mM}(2 \mathrm{ml}$ of $0.5 \mathrm{M})$ & As described in 1.2 \\
\hline HBSS & $100 \mathrm{ml}$ & Invitrogen, USA \\
\hline
\end{tabular}

BSA was filter sterilised (Steritop ${ }^{\mathrm{TM}}$, Millipore) and stored at $4^{\circ} \mathrm{C}$.

\section{Buffers for protein labelling}

\subsection{1 M Sodium bicarbonate buffer}

\begin{tabular}{|l|l|l|}
\hline Item & Volume & Supplier \\
\hline Sodium bicarbonate & $0.42 \mathrm{~g}(\mathrm{MW}=84.08)$ & Sigma-Aldrich, USA \\
\hline $\mathrm{DH}_{2} \mathrm{O}$ & $50 \mathrm{~mL}$ & \\
\hline
\end{tabular}

The buffer was made fresh just before use and filter sterilised with $0.22 \mu \mathrm{m}$ filter (Sartorius, Global Science and Technology Ltd, Germany).

\section{Buffers for protein concentration detection}

\subsection{Bradford reagent}

\begin{tabular}{|l|l|l|}
\hline Item & Volume & Supplier \\
\hline $\begin{array}{l}\text { Coomassie Brilliant Blue } \\
\mathrm{G}\end{array}$ & $50 \mathrm{mg}$ & Sigma-Aldrich, USA \\
\hline Ethanol (95\% pure) & $25 \mathrm{ml}$ & BDH Laboratory supplies, UK \\
\hline $\begin{array}{l}\text { Phosphoric acid }(85 \% \\
\text { pure) }\end{array}$ & $50 \mathrm{ml}$ & Sigma-Aldrich, USA \\
\hline $\mathrm{DH}_{2} \mathrm{O}$ & $375 \mathrm{ml}$ & \\
\hline
\end{tabular}

The buffer is a light brown in colour. The buffer is filtered via a $0.22 \mu \mathrm{m}$ filter (Sartorius, Global Science and Technology Ltd, Germany) to remove the reagent of blue components before use.

\section{Buffers for cytokine detection}

\subsection{Bioplex assay buffer}

\begin{tabular}{|l|l|l|}
\hline Item & Volume & Supplier \\
\hline PBS & $500 \mathrm{ml}$ & As described in 1.1 \\
\hline BSA & $0.1 \%$ & Pierce, USA \\
\hline Tween20 & $0.05 \%$ & Sigma-Aldrich, USA \\
\hline Sodium azide & $0.005 \%$ & Invitrogen, USA \\
\hline EDTA & $2.5 \mathrm{mM}$ & As described in 1.2 \\
\hline
\end{tabular}

The buffer was stored at $4^{\circ} \mathrm{C}$ and brought to room temperature 30 minutes before use. 


\section{Solutions for Griess reaction}

\subsection{Griess solution A}

\begin{tabular}{|l|l|l|}
\hline Item & Volume & Supplier \\
\hline Sulfanilamide & $1 \%$ & BDH Laboratory supplies, UK \\
\hline Phosphoric acid & $2.5 \%$ & BDH Laboratory supplies, UK \\
\hline
\end{tabular}

Stored at room temperature protected from light.

\subsection{Griess solution B}

\begin{tabular}{|l|l|l|}
\hline Item & Volume & Supplier \\
\hline $\begin{array}{l}\text { N-(1-naphthyl) } \\
\text { ethylenediamine }\end{array}$ & $0.1 \%$ & ABCR GmbH, Germany \\
\hline Phosphoric acid & $2.5 \%$ & BDH Laboratory supplies, UK \\
\hline
\end{tabular}

Stored at room temperature protected from light.

\subsection{Nitrite standard}

\begin{tabular}{|l|l|l|}
\hline Item & Volume & Supplier \\
\hline Sodium nitrite & $0.1 \mathrm{M}$ & BDH Laboratory supplies, UK \\
\hline $\mathrm{DH}_{2} \mathrm{O}$ & $1 \mathrm{ml}$ & \\
\hline
\end{tabular}

The buffer was filter sterilised (Steritop ${ }^{\mathrm{TM}}$, Millipore) and stored at room temperature. 